\title{
Neuropathology of schizophrenia : a new vistas
}

\author{
Citation for published version (APA):
}

Kreczmanski, P. (2009). Neuropathology of schizophrenia : a new vistas. [Doctoral Thesis, Maastricht University]. Maastricht University. https://doi.org/10.26481/dis.20090513pk

Document status and date:

Published: 01/01/2009

DOI:

10.26481/dis.20090513pk

Document Version:

Publisher's PDF, also known as Version of record

\section{Please check the document version of this publication:}

- A submitted manuscript is the version of the article upon submission and before peer-review. There can be important differences between the submitted version and the official published version of record.

People interested in the research are advised to contact the author for the final version of the publication, or visit the DOI to the publisher's website.

- The final author version and the galley proof are versions of the publication after peer review.

- The final published version features the final layout of the paper including the volume, issue and page numbers.

Link to publication

\footnotetext{
General rights rights.

- You may freely distribute the URL identifying the publication in the public portal. please follow below link for the End User Agreement:

www.umlib.nl/taverne-license

Take down policy

If you believe that this document breaches copyright please contact us at:

repository@maastrichtuniversity.nl

providing details and we will investigate your claim.
}

Copyright and moral rights for the publications made accessible in the public portal are retained by the authors and/or other copyright owners and it is a condition of accessing publications that users recognise and abide by the legal requirements associated with these

- Users may download and print one copy of any publication from the public portal for the purpose of private study or research.

- You may not further distribute the material or use it for any profit-making activity or commercial gain

If the publication is distributed under the terms of Article $25 \mathrm{fa}$ of the Dutch Copyright Act, indicated by the "Taverne" license above, 


\section{Neuropathology of schizophrenia: a new vistas}

Pawel Kreczmanski 
(c) Paweł Kreczmański, Maastricht 2009

ISBN/EAN: 978-90-9024305-4

Cover design by Paweł Kreczmański

Typesetting by Michał Sławiński

Printed in Poland 


\title{
Neuropathology of schizophrenia: a new vistas
}

\author{
proefschrift
}

ter verkrijging van de graad van doctor aan de

Universiteit van Maastricht, op gezag van de

Rector Magnificus, prof. mr. G.P.M.F. Mols, volgens het besluit van de College van Decanen, in het openbaar te verdedigen

op woensdag 13 mei 2009 om 12.00 uur

door

Pawel Kreczmanski 


\section{Promotor}

Prof. dr. W.H.M. Steinbusch

\section{Copromotor}

\section{Dr. C. Schmitz}

\section{Beoordelingscommissie/Evaluation committee}

Prof. dr. JHS. Vles (voorzitter)

Dr. T. Wierzba-Bobrowicz Institute of Psychiatry and Neurology, Warsaw Poland

Prof. dr. M Lammens (Universiteit Nijmegen)

Prof. dr. G. Hopkins (Universiteit Maasticht)

Prof. dr. rer. H. Korr. (universiteit Aachen) 
I came to this world with nothing, And l leave with nothing but love. Anything else is just borrowed. The streets 


\title{
Contents
}

\author{
Chapter $1 \quad 97$ \\ Introduction
}

Chapter 2

19

Volume, neuron density and total neuron number in the caudate nucleus, putamen, nucleus accumbens, mediodorsal nucleus of the thalamus and lateral nucleus of the amygdala in schizophrenia

\section{Chapter 3}

47

Microvessel length density, total length, and length per neuron in the caudate nucleus, putamen, nucleus accumbens, mediodorsal nucleus of the thalamus and lateral nucleus of the amygdala in schizophrenia

\section{Chapter $4 \quad 71$}

Stereologic studies of capillary length density in cortical areas 9 and 24 in schizophrenia

\section{Chapter 5}

Mean cell spacing abnormalities in the neocortex

of patients with schizophrenia

\section{Chapter $6 \quad m$}

Neuronal distribution in the neocortex of schizophrenic patients

\section{Chapter 7}

General discussion and future implications

$\begin{array}{rr}\text { Summary } & 145 \\ \text { Polish summary (polskie streszczenie) } & 147 \\ \text { Dankwoord } & 149 \\ \text { Curriculum vitae } & 151\end{array}$


Introduction 
Schizophrenia is a devastating neuropsychiatric disorder characterized by psychosis, cognitive impairment and social withdrawal (Mueser and McGurk, 2004). It is the most disabling disorder among mental illnesses (Mueser and McGurk, 2004), being listed as the one of the top ten leading causes of disease related disability in the world (WHO, 2001). The molecular and cellular mechanisms involved in schizophrenia are still remaining largely illusive (Tsuang, 2000; Harrison and Weinberger, 2005). A combination of genetic susceptibility and environmental perturbations appears to be necessary for the expression of the disease (Tsuang, 2000; Siever and Davis, 2004; Harrison and Weinberger, 2005). Possibilities range from alterations of highly specific developmental processes at the synaptic level (Harrison, 1999; Lewis and Lewitt, 2002) and abnormalities in myelination (Hakak et al., 2001; Tkachev et al., 2003) to global metabolic changes of brain function (Berman et al., 1986; Andreasen et al., 1992; Middleton et al., 2002). As the function of neurons depends on adequate oxygen and substrate supply, subtle changes in vascular integrity may also be important for the brain of patients with schizophrenia.

\section{Clinical picture and historical perspective of schizophrenia}

The course and outcome of schizophrenia were for the first time systematically investigated by Emil Kraepelin (Kraepelin, 1919). The concept of dementia praecox developed by Emil Kraepelin was based on linking together specific "Bilder" or concepts of madness and making them uniformed. At that time a rapid development of bacteriology gave a useful paradigm for a model of the disease. It was based on the finding that almost every investigated disease was caused by external factors such as bacteria or later discovered viruses. There were then two elements of the equation: the disease and its cause. The same paradigm was considered in psychiatry motivated by spectacular success in the treatment of cerebral syphilis. Kraepelin's original idea was to complete it by adding two important elements that for a long time dominated the traditional view of mental disorders. The first one implies that the mental disease is also a disease of the brain. In other words, there is no mental disease without neuropathological alterations in the brain. The second one was more illusory and called "eigentümliche Schwächezustände" ("peculiar states of mental weakness") by Kraepelin (1919). "Eigentümlich" (which can be translated into English as "peculiar") means in an original sense a state or feature exclusively belonging to something, in this context to an abstractive object or definition. Kraepelin (1919) used the word "eigentümlich" always with respect to "Schwächezustände". Thus, "eigentümliche Schwächezustände" was considered by Kraepelin (1919) as unique only for schizophrenia. Kraepelin (1919) also grouped and simply listed the features of the psychopathology of schizophrenia. Although the importance of Kraepelin's work cannot be underestimated, it was Bleuler (1911), who pushed the concept furthermore and gave it the more adequate name "schizophrenia" (a "shattered mind"; $\sigma \chi i \zeta \omega$ - schizo, to split or to divide and $\varphi \rho \eta \dot{\eta} v$-phren, mind). In his ideas, deeply rooted in a German Enlightenment philosophy, Breuler acquired and fused essence of the Germanic tradition, the idea of the disturbance of the reason as a basis of the disease and association theory of Locke (Locke, 1841). Using associations to determine the classification of symptomatology of 
schizophrenia, Bleuler (1911) rejected the holistic idea of grouping the symptoms without any distinction among them and proposed a system based on two groups of symptoms: basic and accessory symptoms. According to Bleuler (1911) the basic symptoms (known as 4A's: flattened Affect, Autism, impaired Association of ideas and Ambivalence) are "as far as we know typical for schizophrenia while the accessory symptoms can also appear in other diseases".

The current valid diagnostic criteria of schizophrenia are listed in the Diagnostic and Statistical Manual of Mental Disorders (DSM-IV-TR, Fourth Edition) and include two or more of the following basic symptoms present for a period of at least one month duration or less if successfully treated.

The "positive" symptoms include:

- Delusions (false beliefs) despite of invalidating evidence, including:

- delusions of persecution (also known as paranoid; for example, the patient is being watched or followed and someone is trying "to get her/him"),

- delusions of reference (people or other creatures communicating with an affected individual through TV or radio),

- somatic delusions (for example, that something foreign is passing through the body), and

- delusions of grandeur, exaltation or majesty (i.e., that an individual possesses special powers or abilities). An example of a grandiose delusion is believing that the patient is God or Napoleon;

- Hallucinations: visual (seeing things that are not there), auditory (hearing voices that other people cannot hear), tactile ( something is touching your skin that isn't there), olfactory (not smelling the same that other people smell) or gustatory (tasting things that do not exist) experiences;

- Disorganized speech in form of incoherence and frequent derailment (also described as "word salads" or rambling monologues); and

- Grossly disorganized or catatonic behaviour (patients demonstrate variable conditions of stupor or inactivity and either rigidity or flexibility of the extremities).

The "negative" symptoms include:

- Alogia, or the poverty of speech;

- Affective flattening (which is the reduction or difficulty to initiate and persist in goal directed behaviour; for example, no longer being interested in activities that the person used to show enthusiasm for).

In addition, lack of emotion, low energy, lack of interest in life, low motivation, inappropriate social skills or lack of interest or ability to socialize with other people and social isolation has been observed in schizophrenia.

The third group of symptoms refers to intellectual and cognitive impairments and include disorganized thinking, slow thinking, difficulty in understanding, poor concentration, poor memory, difficulty in expressing thoughts and difficulty integrating thoughts, feelings and behaviour.

Although the symptomatology of schizophrenia includes a wide spectrum of cognitive alterations there is no pathognomonic sign of the disease (Kraepelin, 1919). Rather the 
diagnosis is based on a profile of symptoms and the clinical course of the patient. Moreover, the severity of different symptoms can vary across patients and through the course of the illness (Harrison et al., 2001). The significant heterogeneity in clinical manifestations and the course of schizophrenia is also reflected in the complexity of its epidemiology.

\section{Epidemiology}

In general, epidemiological data raise a very important issue over the variations in the diagnostic criteria and ascertainment methods which are often the source of obscrved differences and discrepancies. Accordingly, epidemiological data about neuropsychiatric disorders should be analyzed with some caution.

The incidence of schizophrenia is approximately $8-40 / 100,000 /$ year and is relatively similar across continents and cultures (Sartorius et al., 1986). There is a higher incidence associated with migration and urbanity (Jablensky et al,, 1992). The point of prevalence reaches the value of 2-10/1,000 including pockets of high and low prevalence (Dohrenwend et al., 1992), with a higher prevalence among lower socio-economical classes (Crow, 2007).

The risk of inception lies between 25 and 35 years for men and women (Haefner et al., 1989) and usually does not extend beyond the age cut-off at 54-59 ycars for the late-onset schizophrenia (Jablensky, 2000). In approximately $75 \%$ of the cases, the first non-specific psychotic episode, diagnosed at mean age of 24.0 years, is preceded by a prodromal phase of several year's' duration (Hambrecht et al., 1994). During this phase non-specific symptoms are predominant. At a mean age of 25.5 years delusions of reference and negative symptoms appear. The first positive symptoms are usually present at mean age of 29.0 years, reaching clinical climax at mean age of 30.1 years, just before the first hospitalization at mean age of 30.3 years (Haefner et al., 1992).

Sex differences, reported for the first time by Kraepelin (1919), are manifested in several different ways. In general, the earlier age at onset and incidence of brain abnormalities are more frequent in men while women have better premorbid performance, less disability and higher possibility for a remitting course (Jablensky, 2000). According to the Jablensky et al., (1992), men are 3-4 years younger than women at the time of the first hospitalization. Nevertheless, sex differences at onset as well as during the course of the disease seem to reflect normal sexual brain dimorphism rather than sex-linked etiological mechanism (Jablensky, 2000).

\section{Genetics}

Schizophrenia is a highly heritable mental illness. Genetic factors contribute up to $80 \%$ to the susceptibility of the disease (Cannon et al., 1998). Gottesman (1991) showed that three degree relatives share $12.5 \%$ of their genetic material and show a risk of $2 \%$ to develop schizophrenia (see also Fig. 1). Half-siblings share respectively $25 \%$ of their genom and show a risk of $6 \%$ to develop schizophrenia. Dizygotic twins with $50 \%$ of shared genes show $9 \%$ of risk to develop the disease, whereas monozygotic twins show a risk near $50 \%$ (Fig. 1). Comparison of the rates between monozygotic vs. Dizygotic twins ( $48 \%$ vs. $16 \%$ ) 
in a meta-analysis based on the data from ongoing 30 years of European studies (Gottesman, 1993) clearly demonstrates genetic heterogeneity of schizophrenia.

The molecular genetic approaches which emerged in the late 1980's failed to find allelic variants conferring susceptibility of schizophrenia (Owen et al., 2004). No single gene, even with an incomplete penetration, was found. Because the recurrence risk decreases too rapidly with an increase of the genetic distance, the transmission of schizophrenia, like other common disorders, is complex and non-Mendelian (McGuc and Gottesman, 1989). However, analyses of positional genes produced promising results in linkage genes studies. Two of those, dysbindin (DTNBP1) (Straub et al., 2002) and neuregulin (NRG1) (Stefansson et al., 2002) have been replicated in at least two independent studies (Schwab et al., 2003, Williams et al., 2003).

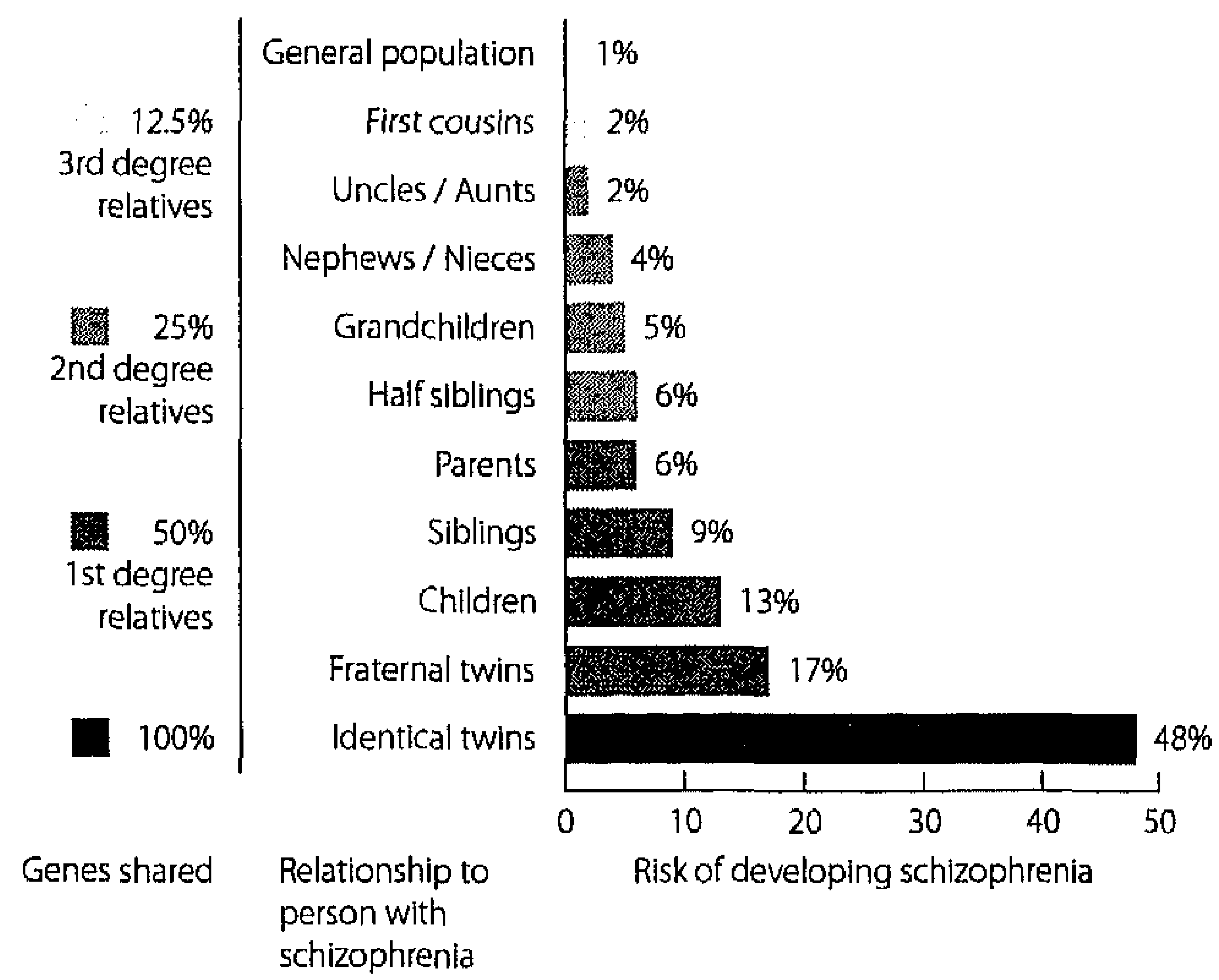

Fig. 1 Population genetics of schizophrenia (taken from Gottesman, 1991).

\section{Environmental risk factors}

The examination of genetic risk factors in association with environmental risk factors, especially developmental abnormalities such as pregnancy and delivery complications, provides valuable information on antecedent states for schizophrenia (Buka and Fan, 1999). Patients with schizophrenia encounter a significantly greater number of labour and delivery complications at birth than in the control group (Geddes and Lawrie, 1995). Among those complications one of the most significant is pre-eclampsia which is responsible for inducing foetal hypoxia. A nine-fold increase in the risk of subsequent schizophrenia has been associated with pre-eclampsia (Kendell et al., 1996; Susser et al., 1996). Moreover, the severity of prenatal hypoxia and the risk of schizophrenia seem to be correlated in a dosedependent manner in the offspring of mothers with schizophrenia (Cannon, 1996). On 
the other hand, delivery complications were unrelated to the development of schizophrenia in the offspring of parents who did not have schizophrenia (Cannon et al., 1993). Several other environmental factors have been associated with schizophrenia, including first and second trimester maternal starvation (Susser et al., 1996), anemia (Kendell 1996), chronic pulmonary disease (Kendell et al., 1996), diabetes (Kendell et al., 1996), and rhesus incompatibility (Dalman et al., 1999) as well as low birth weight and premature delivery (Dalman et al., 1999). Maternal viral infections are also known to be associated with increased risk of schizophrenia (Mednick et al., 1988).

\section{Neurodevelopmental and neurodegenerative hypotheses of schizophrenia}

Alterations of the highly specific developmental processes have generated a lot of interest among neuroscientists investigating schizophrenia (Harrison, 1999; Lieberman, 1999; Tsuang, 2000; Lewis and Lewitt, 2002; Murray et al., 2004). Many genetic and environmental risk factors of schizophrenia appear either prior to or around the time of birth (Jones and Cannon, 1998) leading to disruption of early postnatal brain development and causing expression of the symptoms in adolescence and adulthood. This assumption was devcloped into the neurodevelopmental hypothesis of schizophrenia.

According to the neurodevelopmental hypothesis of schizophrenia abnormal genetics and epigenetics together with gestational and/or perinatal trauma amplify and influence abnormalities in development of the brain of patients with schizophrenia (Weinberger, 1987). Thus, postpubertal onset of the disease is attributed to the continuing development of brain throughout adolescence. Neural networks do not develop properly at a certain time point during pregnancy and patients develop psychosis as a result of long standing abnormalities interacting with environmental factors. Evidence suggesting the existence of neuropathology before the onset of the disease include:

- congenital and minor physical alterations such as abnormalities in the capillary system of the fingernail folds which have been found in patients affected by the disorder (Curtis et al., 1999) and reduction of the vasodilatory response to methylnicotinate application in the skin of patients with schizophrenia (Ross et al., 2004);

- childhood neuromotor, social and cognitive abnormalities;

- enlargement of the lateral ventricles (Harrison, 1999; Wright et al., 2000; Shenton et al., 2001) as well as bilateral reduction of the total volume of the cerebral cortex (Harrison, 1999; Shenton et al., 2001) which is not related to the patient's age;

- absence of gliosis and cytoachitectural abnormalities in cortical and subcortical regions of the brain of patients with schizophrenia in postmortem studies (Falkai et al., 1999).

One of the underlying mechanisms causing these cytoarchitectural abnormalities, which have long been suspected to cause widespread neuronal dysfunction in schizophrenia, is hypoxia (Kety, 1959a; 1959b).

Despite plethora of data there is however no direct evidence for neurodevelopmental lesions directly responsible for schizophrenia. The disease is also not inevitably irreversible. 
Antipsychotic medication can successfully improve symptoms and change the course and duration of the illness. Moreover, there is no explanation how an developmental event, latent for two decades, can not only trigger schizophrenia but also be responsible for heterogeneous and often progressive clinical courses (McClure and Lieberman, 2003). Thus a restricted neurodegenerative process cannot be fully rejected.

In a neurodegenerative approach abnormalities in brain maturation and genetic and/or epigenetic events lead to neurodegenerative process that can be associated with progressive course of the illness. Evidence includes:

- progressive clinical course;

- age related progressive loss of synapses (Lieberman, 1999); and

- progression of cognitive dysfunction.

In this context loss of cognitive functions, chronicity and onset of schizophrenia resemble other neurodegenerative diseases such as Alzheimer's disease or Huntington's disease. These two alternative hypotheses (i.e., neurodevelopmental vs. Neurodegenerative), although regarded incompatible, are not mutually exclusive. Rather the possibility that neurodegeneration occurs after the onset of the disease cannot be completely ruled out. Since psychotic episodes that are manifested after the onset can produce a neurotoxic effect, they could also have a detrimental effect on the brain triggering another, yet unknown, process (Wyatt, 1995; McGlashan and Johannessen, 1996) (see Fig. 2).

Gene predisposition

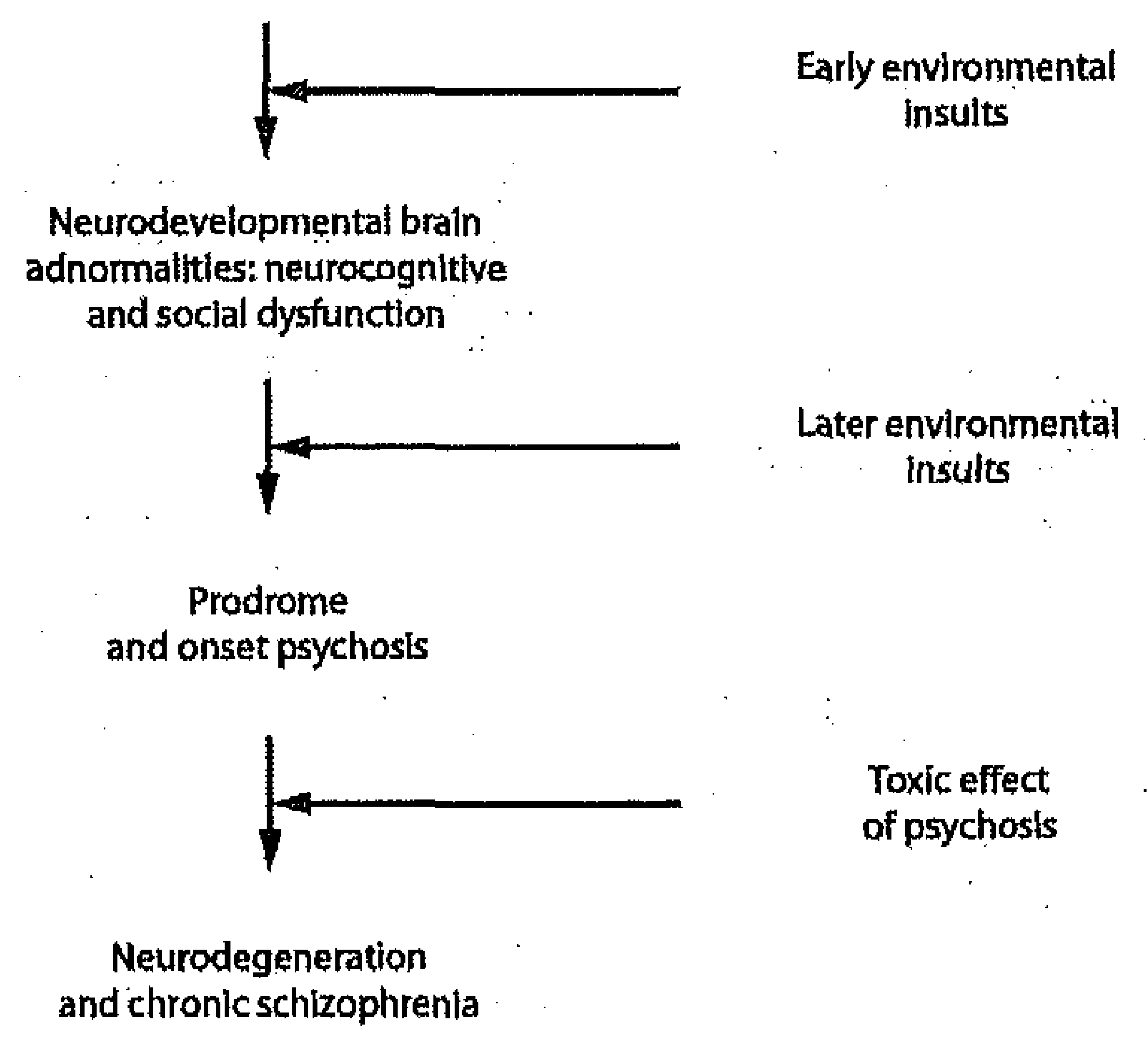

Fig. 2. Processes that have been indicated to play a pivotal role in development, onset and course of schizophrenia (according to Tsuang, 2000). 


\section{Hypoxia and schizophrenia}

As the function of neurons depends on adequate oxygen and substrate supply, subtle changes in vasculature integrity may also be important for the brain of patients with schizophrenia. It has been argued that a subtle metabolic deficit such as hypoxia would be sufficient to cause widespread neuronal dysfunction (Kety, 1959a; 1959b). Furthermore, a number of abnormalities in the coupling between neuronal acrivity and cerebral blood flow were described in patients with schizophrenia (Mcyer-Lindenberg et al., 2002; Hill et al., 2004,). These changes were attributed to primary neuronal dysfunction, although subtle changes in the capillary system were not excluded. Abnormalities in the capillary system of the fingernail folds was found in patients affected by the disorder (Curtis et al., 1999), and the vasodilatory response to methylnicotinate application in the skin is reduced in patients with schizophrenia (Ross et al., 2004). A study employing a combined transcriptomics, proteomics and metabolomics approach found evidence for mitochondrial dysfunction, compromised brain metabolism, and oxidative stress in the frontal cortex of patients with schizophrenia (Prabakaran et al., 2004) thereby confirming and expanding earlier reports (Berman et al., 1986; Andreasen et al., 1999; Middleton et al., 2002). Prabakaran et al. (2004) hypothesized that the observed alterations can be explained by abnormalities of the microvasculature in schizophrenia.

\section{Postmortem studies and modern imaging techniques}

Modern imaging techniques such as computerized tomography (Johnstone et al., 1976) and particularly magnetic resonance imaging (MRI) have led to a revival of postmortem investigations on alterations in the brain of patients with schizophrenia over the last decades. Based on meta-analyses of several MRI and CT investigations there is now a strong body of evidence in favor of an enlargement of the lateral ventricles (Harrison, 1999; Wright et al., 2000; Shenton et al., 2001) as well as of a bilateral reduction of the total volume of the cerebral cortex (Harrison, 1999; Shenton et al., 2001).

Postmortem investigations revealed subtle alterations in several cortical regions in schizophrenia such as the prefrontal, entorhinal and anterior cingulate cortices (Harrison, 1999; Taminga et al., 2000; Selemon, 2001) as well as in subcortical regions such as the caudate nucleus, putamen and nucleus accumbens (Bogerts, 1984; Beckmann and Lauer, 1997; Lauer and Beckmann, 1997), the mediodorsal nucleus of the thalamus (Pakkenberg, 1990; Popken et al., 2000; Young et al., 2000; Byne et al., 2002; Cullen et al., 2003; Dorph-Petersen et al., 2004; Danos et al., 2005) and the amygdala (Bogerts, 1984) with respect to volume, total neuron number and neuron density. However, methodological differences such as the lack of standardization and normalization of applied procedures, investigated parameters and above all availability of the investigated samples from postmortem brains of patients with schizophrenia and matched controls were to blame for the discrepancy and divergence of published results (Harrison, 1999). A possible solution to this unsatisfactory situation is the analysis of several regions in brains from patients with schizophrenia and matched controls with state-of-the-art quantitative histological tech- 
niques in one study. Moreover, general observations on the macrovasculature in the brain of patients with schizophrenia were performed (Arnold and Trojanowski, 1996), but there are no systematic, design-based stereological studies on the vascular system in this disease. For this purpose I performed capillary length density measurements within several cortical and subcortical regions in postmortem brains from patients with schizophrenia and matched controls with the recently developed "Space balls" method (Calhoun and Mouton, 2000; Mouton et al., 2002; Schmitz and Hof, 2005).

Accordingly, a new methodological approach was performed in the present study to shed new light on the neuropathology of schizophrenia, including (among many others) the following innovative key elements:

- A large and carefully selected sample of postmortem brains from 13 male patients with schizophrenia and age-matched male controls was analyzed, including full medical records and autopsy records. All patients with schizophrenia met the Diagnostic Statistical Manual, 4th revision (DSM-IV) and International Statistical Classification of Diseases and Related Health Problems, 10th revision (ICD-10) diagnostic criteria.

- Tissue processing was performed as follows: both hemispheres were cut into serial 600-700 $\mu \mathrm{m}$-thick coronal sections (Heinsen and Heinsen, 1991). From each hemisphere, every second or third section was stained with gallocyanin. Adjacent sections not stained with gallocyanin were selected, and small pieces containing the desired brain region were cut out for investigation with immunohistochemistry (Kreczmanski et al., 2005; 2007).

- "Space balls" technology (Schmitz and Hof, 2005): after delineation of the investigated brain regions, the sections processed with immunohistochemistry were brought into focus. Then hemispheres with radius of $30 \mu \mathrm{m}$ (generated with a special stereology software [Stereoinvestigator; MBF Bioscience, Williston, VT, USA) while focussing through the section thickness) were systematically and randomly placed within the sections through the regions of interest. The capillary length density was obtained from the total number of intersections between the hemispheres and the capillaries as described considering the area of the delineated region of interest and the actual section thickness after tissue processing using the following equation:

$$
C L D=\frac{2 \times \sum i s\left(D_{X} \times D_{Y} \times t\right)}{2 \times \pi \times r^{3}} \times \frac{1}{V}
$$

where $C L D$ represents the capillary length density, $\sum$ is the total number of intersections between the hemispheres and the capillaries, $D_{X}$ and $D_{Y}$ are the distances between the midpoints of the centers of the hemispheres in directions $X$ and $Y, \mathrm{t}$ is the actual average section thickness after histologic processing, $r$ the radius of the hemispheres and $V$ the investigated tissue volume. 
- Minicolumn analysis: minicolumns are thought to be the basic cytoarchitectural entity of the mammalian neocortex, also known as cortical microdomain. Although the functional significance of minicolumns remains controversial (Jones, 2000; Hutsler and Galuske, 2003) they have been proposed as a primary cortical information processing unit (Mountcastle, 1997). Minicolumns are (two-dimensionally defined) detected in Nissl-stained sections in linear arrangements, perpendicular to the pial surface, of the neurons and a presence of a cell-free space on both sides of the column core (Buxhoeveden and Casanova, 2002b) creating a basic input/output unit that overlaps internal processing chains (Mountcastle, 1997). It has been hypothesised that middle layered neurons excited by incoming stimuli from thalamic afferents with only small differences in latency are tapered in vertical connections to both superficial and deep layers (Jones, 2000).

The analyses were performed under the hypothesis that a distinct pattern of histological alterations would emerge from these cortical and subcortical areas known to be involved in the neuropathology of schizophrenia. Specifically,

- Chapter 2 of this thesis describes morphological alterations of the cytoarchitecture in five subcortical regions of the brain of patients with schizophrenia (caudate nucleus, putamen, nucleus accumbens, mediodorsal nucleus of the thalamus, and lateral nucleus of the amygdala), investigated with state-of-the-art design-based stereological techniques (focussing on volumes, neuron densities and total neuron numbers) (published in Kreczmanski et al., 2007);

- Chapters 3 and 4 describe results of investigations on microvasculature abnormalities suggested in the brain of patients with schizophrenia (published in Kreczmanski et al., 2005; 2008); and

- Chapters 5 and 6 describe minicolumnar abnormalities in the neocortex of the patients with schizophrenia, focussing on alterations in mean cell spacing (published in Casanova et al., 2005) and in the distribution of neurons in the neocortex (published in Casanova et al., 2008). 


\title{
Chapres 2
}

\section{Volume, neuron density and total neuron number in five subcortical regions in schizophrenia}

\author{
Pawel Kreczmanski ${ }^{1,2}$, Helmut Heinsen ${ }^{3}$, Valentina Mantua ${ }^{1,6,7}$, Fritz Woltersdorf $f^{4}$ \\ Thorsten Masson ${ }^{5}$, Norbert Ulfig ${ }^{4}$, Rainald Schmidt-Kastner', Hubert Korr, 1,25 \\ Harry W. M. Steinbusch ${ }^{1,2}$, Patrick R. Hof and Christoph Schmitz ${ }^{1,2}$
}

' Department of Psychiatry and Neuropsychology, Division of Cellular Neuroscience, Maastricht University

2 European Graduate School of Neurosclence (EURON), Maastricht, The Netherlands ${ }^{3}$ Morphological Brain Research Unit, University of Wuerzburg, Wuerzburg

${ }^{4}$ Department of Anatomy, University of Rostock, Rostock, Germany,

${ }^{5}$ Department of Anatomy and Cell Biology, RWTH Aachen University, Aachen 'Department of Psychiatry, Section of Clinical Neuropharmacology, Institute of Psychiatry, Kings College London, London, UK

'Department of Psychiatric Sciences and Psychological Medicine, Psychiatric Clinic ill, University of Rome La Sapienza, Rome, Italy

${ }^{8}$ Department of Neuroscience, Mount Sinai School of Medicine, New York, NY, USA 


\begin{abstract}
Several studies have pointed to alterations in mean volumes, neuron densities and total neuron numbers in the caudate nucleus $(\mathrm{CN})$, putamen, nucleus accumbens (NA), mediodorsal nucleus of the thalamus (MDNT) and lateral nucleus of the amygdala (LNA) in schizophrenia. However, the results of these studies are conflicting and no clear pattern of alterations has yet been established in these subcortical regions, possibly due to differ- ences in quantitative histological methods used as well as differences in the investigated case series. The present study investigates these subcortical regions in both hemispheres of the same post-mortem brains for volume, neuron density and total neuron number with high-precision design-based stereology. The analysed case series consisted of 13 post-mortem brains from male schizophrenic patients [age range: 22-64 years; mean age $51.5 \pm 3.3$ years $($ mean $\pm S E M)]$ and 13 age-matched male controls (age range: $25-65$ years; mean age $51.9 \pm 3.1$ years). A general linear model multivariate analysis of variance with diagnosis and hemisphere as fixed factors and illness duration (schizophrenic patients) or age (controls), post-mortem interval and fixation time as covari- ates showed a number of statistically significant alterations in the brains from schizophrenic patients compared with the controls. There was a reduced mean volume of the putamen $[-5.0 \%$ on the left side (l) and $-4.1 \%$ on the right side (r)] and the LNA (1: $-12.1 \%, \mathrm{r}:-17.6 \%)$, and a reduced mean total neuron number in the CN (1: $-10.4 \%, \mathrm{r}:-10.2 \%)$, putamen (1: $-8.1 \%, \mathrm{r}:-11.6 \%)$ and the LNA (1: $-15.9 \%, \mathrm{r}:-16.2 \%)$. These data show a previously unreported, distinct pattern of alterations in mean total neuron numbers in identified subcortical brain regions in a carefully selected sample of brains from schizophrenic patients. The rigorous quantitative analysis of several regions in brains from schizophrenic patients and matched controls is crucial to provide reliable information on the neuropathology of schizophrenia as well as insights about its pathogenesis.
\end{abstract}




\title{
Abbreviations
}

\author{
ABNA - accessory basal nuclei of the amygdala \\ BNA - basal nuclei of the amygdala \\ CGM - cortical grey matter \\ $\mathrm{CN}$ - caudate nucleus \\ LNA - lateral nucleus of the amygdala \\ MDNT - mediodorsal nucleus of the thalmus; \\ $\mathrm{NA}$ - nucleus accumbens
}

\section{Introduction}

Schizophrenia is a devastating neuropsychiatric disorder that presents with a variety of cognitive disturbances in attention, working memory, verbal production and response monitoring and inhibition. Equally diverse are the putative mechanisms which translate deficits into schizophrenic pathology, and the anatomical substrates ofron number in schizophrenia both the deficits and the pathology (Andreasen et al., 1999). Modern imaging techniques have indicated various discrete morphological alterations in the brain from schizophrenic patients. Based on meta-analyses, there is now a strong body of evidence in favour of an enlargement of the lateral ventricles (Harrison, 1999; Wright et al., 2000; Shenton et al., 2001; Kasai et al., 2002), as well as of a reduction of the total volume of the cerebral cortex bilaterally in schizophrenia (Harrison, 1999; Shenton et al., 2001).

Post-mortem studies have revealed subtle neuropathological abnormalities in several cortical regions in schizophrenia such as the prefrontal, entorhinal and anterior cingulate cortices (for review see Harrison, 1999; Taminga et al., 2000; Selemon, 2001), as well as in subcortical regions such as the caudate nucleus $(\mathrm{CN})$, putamen and nucleus accumbens (NA) (Bogerts, 1984; Beckmann and Lauer, 1997; Lauer and Beckmann, 1997), the mediodorsal nucleus of the thalamus (MDNT) (Pakkenberg, 1990; Popken et al., 2000; Young et al., 2000; Byne et al., 2002; Cullen et al., 2003; Dorph-Petersen et al., 2004; Danos et al., 2005) and the amygdala (Bogerts, 1984). However, many of the reported data are divergent if not mutually contradictory and no clear pattern of histological alterations has yet been established in schizophrenia. Such discrepancies can be explained by methodological differences, investigated parameters and particularly the available samples of brains from schizophrenic patients and controls (Harrison, 1999).

A possible solution to this situation is the analysis of several regions in brains from schizophrenic patients and matched controls with state-of-the-art quantitative histological techniques. We have used this approach recently in investigations of mean cell spacing abnormalities in the neocortex (Casanova et al., 2005) and capillary length densities in the frontal cortex (Kreczmanski et al., 2005) in a carefully selected sample of post-mortem brains from male schizophrenic patients and age/gender-matched controls. In the pres- 
ent study, we have continued the analysis of these brains by investigating five subcortical regions [CN, putamen, NA, MDNT and lateral nucleus of the amygdala (LNA)] with a high-precision design-based stereology approach for possible alterations in volumes, neuron densities and total neuron numbers. The analyses were performed under the hypothesis that a distinct pattern of histological alterations would emerge from these sub-cortical areas known to be involved in the neuropathology of schizophrenia.

\section{Material and methods}

\section{Brain specimens}

This study was performed on the same post-mortem brains (both hemispheres) from 13 male schizophrenic patients [mean age $51.5 \pm 3.3$ years; mean post-mortem interval (time between death and autopsy) $27.5 \pm 6.0 \mathrm{~h}$; mean fixation time $912 \pm 372$ days; data given as mean $\pm S E M]$ and from 13 age-matched male controls (mean age $51.9 \pm 3.1$ years; mean post-mortem interval $23.7 \pm 3.8 \mathrm{~h}$; mean fixation time $247 \pm 53$ days) that were investigated in our previous studies for mean cell spacing abnormalities in the neocortex (Casanova et al., 2005) and for capillary length densities in the frontal cortex (Kreczmanski et al., 2005). The age of the patients, illness duration, clinical diagnoses, causes of death, the post-mortem interval and the fixation time are summarized in Table 1. The schizophrenic patients did not differ from the controls with respect to mean age (Student's two- tailed t-test; $P=0.946)$, mean post-mortem interval $(P=0.581)$ and mean fixation time $(P=0.089)$. All schizophrenic cases had been patients either in German university hospitals or in German State psychiarric hospitals (six hospitals including one university hospital in which some control patients were also treated for non-psychiatric or neurological illnesses) and full clinical records were available. All controls had been patients either in German university hospitals or in German local district hospitals (five hospitals including one university hospital in which schizophrenic patients were also treated). Records from autopsy (including a summary of the medical history) were available for all schizophrenic cases and all controls. All pathologists involved in the autopsies were instructed by H.H. and adhered to identical handling and processing conditions of the brains. All schizophrenic patients met the Diagnostic Statistical Manual,4th revision (DSM-IV) and International Statistical Classification of Diseases and Related Health Problems, 10th revision (ICD-10) diagnostic criteria. The clinical notes were assessed by two experienced clinical psychiatrists to ensure that the brains from the controls were free from psychopathology, and for clear evidence that the diagnosis of schizophrenic patients was concordant with DSM-IV criteria for schizophrenia. The mean age at onset was $22.9 \pm 1.5$ years. Exclusion criteria for both schizophrenic patients and controls comprised neurological problems that required intervention or interfered with cognitive assessment (e.g. stroke with aphasia), history of recurrent seizure disorder, history of severe head injury with loss of consciousness, diabetes 
mellitus with free plasma glucose $4200 \mathrm{mg} / \mathrm{dl}$ and history of self-administered intoxication. Schizophrenic patients and controls were similar in terms of the ethnic backgrounds. However, they were not fully matched for socioeconomic status and education, which would have placed severe constraints on our sample. Moreover, all schizophrenic patients were subjected to long-term treatment with typical neuroleptics (because of the fact that most of the patients were not hospitalized throughout the duration of their illness, and, therefore, the clinical records did not cover fully the entire medication histories, it was not possible to calculate lifetime medication exposures). In all of the cases, autopsy was performed after consent was obtained from a relative according to the laws of the Federal Republic of Germany. The use of these autopsy cases for scientific investigations as outlined here has been approved by the relevant institutional review boards. Brains were fixed by immersion in $10 \%$ formalin (one part commercial $40 \%$ aqueous formaldehyde in nine parts $\mathrm{H}_{2} \mathrm{O}$ ) prior to histological processing.

\section{Tissue processing}

The brainstem with the cerebellum was separated from the forebrain at the level of the rostral pons, and the hemispheres were divided mediosagittally. Then, both hemispheres were cut into serial 600-700 mm-thick coronal sections as previously described (Heinsen and Heinsen, 1991). Briefly, the hemispheres were cryoprotected in a mixture of glycerol-dimethylsulphoxide-formalin after carefully removing the meninges and pial vessels, embedded in gelatin, deeply frozen at $-60^{\circ} \mathrm{C}$ and serially sectioned using a cryomicrotome (Jung, Nussloch, Germany). One brain (C7) was embedded in celloidin as recently described (Heinsen et al., 2000) and was cut into serial 440-500 tm-thick coronal sections using a sliding microtome (Polycut, Cambridge Instruments, UK). From each hemisphere, every second or third section was stained with gallocyanin (a Nissl stain) as previously described (Heinsen and Heinsen, 1991). The remaining sections were stored in formalin for up to 10 years. From all the brains of the schizophrenic patients and controls older than 40 years, sections through the central portion of the entorhinal and transentorhinal cortex that were not stained with gallocyanin were labelled with the Gallyas method to detect neurofibrillary changes (Heinsen et al., 1989). Neurofibrillary tangles were very rarely detected in the transentorhinal and entorhinal cortex on Gallyas-stained sections, compatible with Braak's stage I (Braak and Braak, 1995). Furthermore, each section was coded and controlled for the absence of tumours, infarcts, heterotopias, signs of autolysis, staining artefacts and gliosis. Fixation and tissue processing were performed at the Morphological Brain Research Unit, University of Wuerzburg (Germany) under identical conditions for all brains (except for brain C7 that was embedded in celloidin instead of gelatin).

\section{Investigated brain regions}

The CN, putamen, NA, MDNT and LNA were investigated for their volume, total number of neurons and neuron density. Delineations of these regions were performed according to established criteria in the literature (CN, putamen and NA: Brockhaus, 1942; Lauer 
and Heinsen, 1996; Holt et al., 1999; Lauer et al., 2001; MDNT: Dewulf, 1971; Hirai and Jones, 1989; Jones, 1997; Heinsen et al., 1999; Popken et al., 2000; Dorph- Petersen et al., 2004; LNA: Braak and Braak, 1983; Sims and Williams, 1990; Sorvari et al., 1996; Schumann and Amaral, 2005). In the case of the MDNT, the magnocellular, parvocellular and densocellular regions as well as the caudodorsal subdivision were included in the delineations, as also done by Popken et al. (2000), Byne et al. (2002) and Dorph-Petersen et al. (2004) (Fig. 1). In addition, the volumes of the cortical grey matter (CGM), the basal and accessory basal nuclei of the amygdala (BNA and ABNA) were determined. Delineations of the BNA and ABNA also followed the criteria established by Braak and Braak (1983), Sims and Williams (1990), Sorvari et al. (1996) and Schumann and Amaral (2005).

The boundaries of the investigated brain regions were identified on all sections showing the corresponding region using an Olympus SZX9 stereo microscope (Olympus; Tokyo, Japan) and were marked on the back side of the glass slides with a felt-tip pen. Identification and delineation of boundaries was performed by H.H. (CGM, CN, putamen), V.M. (NA), T.M. (MDNT) and F.W. (LNA, BNA and ABNA). Cross-validation (and, if necessary, slight modifications of the delineations) was performed by C.S. (CGM, CN, putamen) and C.S., H.H. and P.R.H. (all other brain regions).

\section{Stereological analyses}

Stereological analyses were performed with a stereology workstation, consisting of a modified light microscope [Olympus BX50 with PlanApo objective $1.25 \times$ [numerical aperture (N.A.) = 0.04) 1 and UPlanApo objectives $10 \times($ N.A. $=0.4), 20 \times($ oil; N.A. $=0.8)$ and $40 \times$ (oil; N.A. = 1.0); Olympus, Tokyo, Japan, motorized specimen stage for automatic sampling (Ludl Electronics; Hawthorne, NY, USA) CCD colour video camera (HV-C20AMP; Hitachi, Tokyo, Japan) and stereology software StereoInvestigator, MicroBrightField, Williston, VT, USA). Volumes of brain regions were analysed using Cavalieri's principle [Cavalieri, 1635; see also Fig. 2 in Schmitz and Hof (2005)], by determining the projection area of a given brain region on each section showing this region, summing the data from all sections, and multiplying this value with the interval of selecting sections for staining with gallocyanin (two or three; see above) and the average actual section thickness after tissue processing [determined with the calibrated fine adjustment knob of an Olympus BH microscope and a Planapo objective (40x; N.A. 1/4 1.0) as described in Heinsen et al. (1994)1. The projection areas of the CGM were determined with point counting [Gundersen and Jensen, 1987; see also Fig. 1 in Schmitz and Hof (2005)1 as already described in Kreczmanski et al. (2005). In contrast, the projection areas of the subcortical regions were determined by tracing their boundaries on each section on video images displayed on the monitor of the stereology workstation.

Total neuron numbers were evaluated with the optical fractionator [West et al., 1991; see also Figs 1 and 4 in Schmitz and Hof (2005)1. All neurons whose nucleus top came into focus within unbiased virtual counting spaces distributed in a systematic-random fashion throughout the delineated regions were counted. Then, total neuron numbers 
were calculated from the numbers of counted neurons and the corresponding sampling probability. Details of the counting procedure for all investigated brain regions are summarized in Table 2.

In addition, the nearest neighbour distance distribution functions were determined for the neurons in the lateral nucleus of the right amygdala with the nearest neighbour method of the Stereolnvestigator software. For each neuron in the lateral nucleus of the right amygdala that was counted with the unbiased virtual counting spaces, the neuron positioned closest to the counted neuron was detected [as explained in Schmitz et al. (2002)1. Then, the distance between these neurons was calculated in three dimensions. From these individual nearest neighbour distances, the nearest neighbour distance distribution function in the lateral nucleus of the right amygdala was calculated for each investigated brain. Finally, neuron densities were calculated individually for each subcortical region as the ratio of the total neuron number in and the volume of this region.

\section{Statistical analysis}

For both schizophrenic patients and controls, mean and standard error of the mean were calculated for all investigated variables (except for the nearest neighbour distance distribution functions), separately for the left and the right hemispheres. Comparisons between schizophrenic patients and controls were performed using generalized linear model multivariate analysis (MANOVA), with diagnosis and hemisphere as fixed factors and the following variables as covariates: (i) the adjusted illness duration of the schizophrenic patients (calculated as individual age at death minus age at onset plus the mean age at onset of all schizophrenic patients) or the age of the controls, respectively, (ii) the postmortem interval and (iii) the fixation time [note that use of the actual individual illness duration of the schizophrenic patients instead of the adjusted ones as covariate would have caused invalid results of the MANOVA model because there was no illness duration of the controls, and the mean illness duration of the schizophrenic patients was significantly different from the mean age of the controls (Student's two-tailed t-test; $\mathrm{P}<0.001$ ) whereas the mean adjusted illness duration was not $(\mathrm{P}=0.974)]$. For each investigated variable, all investigated brain regions were tested simultaneously. Post hoc tests in the analyses of covariance were performed with linear regression analysis. In all analyses an effect was considered statistically significant if its associated $\mathrm{P}$-value was $<0.05$. Calculations were performed using SPSS (Version 12.0.1 for Windows, SPSS, Chicago, IL, USA). The nearest neighbour distance distribution functions of the ncurons in the lateral nucleus of the right amygdala from the schizophrenic patients and the controls were graphically analysed with empirical distribution function (EDF) plots as explained in detail in Schmitz et al. (2002). The computer simulations necessary to perform these EDF plot analyses were carried out with Microsoft Visual Basic (version 6.0; Microsoft, Redmond, WA, USA); graphical analysis was done with GraphPad Prism (version 4.00 for Windows; GraphPad Software, San Diego, CA, USA). 


\section{Photography}

Photomicrographs shown in Fig. 1A-F and N-S were produced by digital photography using the stereology workstation described above. On average, 120 images were captured for the composite in each Fig. 1A-C and Q-S, and 16 images for the composite in each Fig. ID-F and N-P. These images were made into one montage using the Virtual Slice module of the StereoInvestigator software. Photomicrographs shown in Fig. 1G-M were produced by digital photography using an Olympus DP 70 digital camera attached to an Olympus AX 70 microscope and cellP software (version 2.3; Soft Imaging System, Muenster, Germany). The final figure was constructed using Corel Photo-Paint v.11 and Corel Draw v.11 (Corel, Ottawa, Canada). Only minor adjustments of contrast and brightness were made, without altering the appearance of the original materials.

\section{Three-dimensional reconstructions}

Reconstructions of LNA, BNA and ABNA in the right hemispheres were performed by digitally photographing close-up views of serial sections of the temporal lobe enclosing the amygdaloid complex with a digital single lens reflex camera (Fuji FinePix S2 Pro; Fuji Photo Film Co., Tokyo, Japan) and a $50 \mathrm{~mm}$ macro objective (1:2.8; Sigma, Kanagawa, Japan). Then the stacked photos were imported into Amira software (version 3.1; Mercury Computer Systems; San Diego, CA, USA). The serial sections were aligned manually in the editor window of Amira with the contours of the parahippocampal gyrus, fusiform gyrus, and the surface of the anterior perforated substance serving as reference structures in the alignment of the individual sections. This coarse alignment was supplemented by a omputer-assisted fine-tuned automatic alignment. Afterwards, the outlines of the LNA, BNA and ABNA were identified and manually traced in the image segmentation editor of Amira. With these outlines and the average section thickness, the software generated surface views of the investigated nuclei of the amygdala. Irregular contours were smoothed by additional editors.

\section{Results}

Compared with controls the schizophrenic patients showed a significantly reduced mean volume of the fixed factor Diagnosis in the MANOVA1, the putamen [1: $-5.0 \%, \mathrm{r}:-4.1 \%$; $F(1)=5.864, P=0.0201$, the BNA [1: $-10.6 \%, r:-11.9 \% ; F(1)=4.447, P=0.0421$ and the LNA $[1:-12.1 \%, \mathrm{r}:-17.6 \% ; \mathrm{F}(1)=8.661, \mathrm{P}=0.006$ ] (Fig. 2; Table 3 shows all $\mathrm{P}$-values of the MANOVAs performed). The smaller volumes of the BNA and LNA in the brains from the schizophrenic patients were also visible in three-dimensional reconstructions of the investigated nuclei of the amygdala (Fig. 3). Notably, not only the volume of the BNA and LNA was smaller in the schizophrenic patients than in the controls but also the combined volume of the BNA, LNA and accessory BNA $\left\{-11.3 \%\right.$ on the left side [schizophrenic patients: $621 \pm 24 \mathrm{~mm}^{3}$; 
controls: $700 \pm 28 \mathrm{~mm}^{3}$; mean \pm SEM; P (Student's t-test) $=0.0421$ and -14.7 on the right side [schizophrenic patients: $603 \pm 23 \mathrm{~mm}^{3}$; controls: $706 \pm 29 \mathrm{~mm}^{3} ; \mathrm{P}=0.011$ ] .

With respect to the mean neuron density in the investigated subcortical regions, there were no significant differences between the schizophrenic patients and the controls (Fig. 4). In contrast, the schizophrenic patients had a significantly reduced mean total neuron number in the $\mathrm{CN}[1:-10.4 \%, \mathrm{r}:-10.2 \% ; \mathrm{F}(1)=7.088, \mathrm{P}=0.011]$, the putamen $[1:-8.1 \%$, $\mathrm{r}:-11.6 \% ; \mathrm{F}(1)=8.733, \mathrm{P}=0.005]$ and the LNA $[1:-15.9 \%, \mathrm{r}:-16.2 \% ; \mathrm{F}(1)=6.498$, $P=0.015$ ] compared with controls (Fig. 5).

Furthermore, no significant differences between the left and the right hemispheres were found for the investigated variables in both schizophrenic and control cases (P40.05 for the fixed factor Hemisphere in all MANOVAs performed). The post-mortem interval had a significant effect on the volume of the putamen $[F(1)=9.553, P=0.004]$ and the total neuron number in this brain region $[F(1)=6.899 ; P=0.012]$ (see Fig. $S 1$ in the Supplementary online material). A significant effect was also found for the control patients' age (or the illness duration of the schizophrenic patients, respectively) on the total neuron number in the $\mathrm{CN}[\mathrm{F}(1)=4.197, \mathrm{P}=0.0471$ and the $\mathrm{NA}[\mathrm{F}(1)=4.224, \mathrm{P}=0.0461$. However, post hoc linear regression analysis revealed only a significant, positive correlation between the illness duration and the total neuron number in the left $\mathrm{CN}$ in the brains from the schizophrenic cases $[\mathrm{r} 2=0.349, \mathrm{~F}(1,11)=5.893, \mathrm{P}=0.034]$. Furthermore, disregarding the total neuron numbers in the $\mathrm{CN}$ from the 22-year-old schizophrenic patient, $\mathrm{S} 1$ had no significant effect on the results from the MANOVA but abolished the significant effects in the post hoc linear regression analysis concerning the correlation between the illness duration and the total neuron number in the left $\mathrm{CN}$ in the brains from the schizophrenic cases $[\mathrm{r} 2=0.213, \mathrm{~F}(1,10)=2.704, \mathrm{P}=0.131]$. It can be therefore concluded that the alterations in mean volumes and mean total neuron numbers found in the investigated subcortical regions in the brains from the schizophrenic patients were not caused by the control patients' age (or the illness duration of the schizophrenic patients, respectively), the post-mortem interval or the fixation time of the brains.

The nearest neighbour distance distribution function analysis of neurons in the lateral nucleus of the right amygdala showed no distinct differences between the schizophrenic patients and the controls (sce Fig. S2 in the Supplementary online material). Finally it should be mentioned that the results obtained for the single brain embedded in celloidin (C7) showed no systematic deviation from the results obtained for the other brains (Figs 2, 4 and 5).

\section{Discussion}

The present study revealed a distinct pattern of subtle neuropathological alterations in a carefully selected sample of brains from schizophrenic patients. This previously unrecognized set of alterations comprises reduced volumes of the total CGM, putamen and LNA as 
well as reduced total neuron numbers in the $\mathrm{CN}$, putamen and LNA (note that the analysis of neuron densities showed no differences between schizophrenic patients and controls). Reports in the literature of reduced volumes of the NA and MDNT as well as reduced total neuron numbers in these subcortical regions in schizophrenia could not be confirmed. Furthermore, no correlation between the illness duration of the schizophrenic patients and the observed neuropathological alterations was found. The results from our control cases are in line with previous studies (summarized in Tables 4 and 5). The results obtained for the schizophrenic patients can be compared with data in the literature as discussed below (see also Tables 4 and 5). In addition, a significant decrease in the total CGM volume (-9.3\%; averaged for the left and right hemispheres) was found when comparing the schizophrenic patients with the controls, in agreement with several reports in the literature (e.g. Zipurski et al., 1992, 1997; McCarley et al., 1999; Shenton et al., 2001).

Clinical neuroimaging studies have shown an enlargement of the striatum in relation to treatment with typical neuroleptics (Chakos et al., 1994; Shenton et al., 2001; Lang et al., 2004). This is in contrast to the results of a post-mortem study by Beckmann and Lauer (1997) who reported no differences in mean volumes of the CN and the putamen when comparing schizophrenic patients treated with typical neuroleptics to controls $(-0.3 \%$ and $+1.9 \%$, respectively; $P>0.05$;) as well as to a report by Bogerts (1984) who found a trend towards reduced mean volumes of the $\mathrm{CN}(-4.5 \%$; $\mathrm{P} 40.05)$ and the putamen $(-4.1 \%$; P>0.05) when comparing schizophrenic neuroleptic-naive patients with controls. Notably, several clinical neuroimaging studies reported a decrease in volume of the striatum in drug-naive schizophrenic patients [reviewed in Shenton et al. (2001)], as well as reduced basal ganglia volumes after switching to olanzapine in schizophrenic patients chronically treated with typical neuroleptics (Chakos et al., 1994; Frazier et al., 1996; Lang et al., 2004). Accordingly, increased volume of the striatum in schizophrenia as found in certain clinical neuroimaging studies might reflect a specific, currently unknown action of typical neuroleptics that is reversible by switching to atypical neuroleptics or during histological processing. In a post-mortem study, Beckmann and Lauer (1997) compared nine schizophrenic patients with nine matched controls, and found an increased total neuron number in the $\mathrm{CN}[\mathrm{P}<0.05$ only for the right hemisphere (difference: $+17.5 \%$ ) but not for the left hemisphere (difference: $+15.8 \%) 1$ and a trend towards an increased total neuron number in the putamen $(+9.8 \% ; \mathrm{P}>0.05)$ in the brains of the schizophrenic patients. This is in contrast to the finding of reduced total neuron numbers in the $\mathrm{CN}(-10.2 \%)$ and putamen $-9.9 \%)$ in the brain of the schizophrenic patients in the present study. The reason for this discrepancy is unknown and might be related to differences in the stereological analysis [the number of unbiased virtual counting spaces used to estimate total neuron numbers was considerably lower in the study of Beckmann and Lauer (1997) than in the present study, implying a considerably higher variation in estimated total neuron numbers (Schmitz, 1998; Schmitz and Hof, 2000, 2005)].

Pakkenberg's (1990) early report of a reduction in the mean volume of the NA in schizophrenia by $42 \%$ (and in brain regions in a three-dimensional impression during 
microscopic inspection. The MDNT has been the focus of several studies applying designbased stereological techniques over the last 20 years (Table 5). Pakkenberg (1990), Popken et al. (2000) and Byne et al. (2002) reported a reduced mean volume and a reduced mean total neuron number in the MDNT in the brains of schizophrenic patients compared with controls the mean total neuron number in this nucleus by $50 \%$ ) should be taken cautiously, as was already discussed by Lauer et al. (2001). Pakkenberg's (1990) data are also in contrast to Bogert's (1984) finding of almost no difference in mean volumes of the NA between brains from 14 schizophrenic patients and 13 controls $(+1.2 \%$; $P>0.05)$. The discrepancy between the results of Pakkenberg (1990), those of Bogerts (1984), Lauer et al. (2001) and the present study might be due to differences in stereological design. For instance, Pakkenberg (1990) performed microscopic investigations on 4 tm-thick paraffin sections stained with gallocyanin. However, exact delineations of the borders of subcortical brain regions on such sections might be much more difficult than on 20 tm-thick paraffin sections processed with a myelin stain [as performed by Bogerts (1984)], on 500 tm-thick (Lauer et al., 2001) or on 440-700 tm-thick (present study) sections stained with gallocyanin, facilitating the identification of borders of brain regions in a three-dimensional impression during microscopic inspection.

The MDNT has been the focus of several studies applying design-based stereological techniques over the last 20 years (Table 5). Pakkenberg (1990), Popken et al. (2000) and Byneetal. (2002) reported a reduced mean volume and a reduced mean total neuron number in the MDNT in the brains of schizophrenic patients compared with controls (studies with positive outcome; PO studies), whereas Cullen et al. (2003), Kemether et al. (2003), Dorph-Petersen et al. (2004), Danos et al. (2005) and the present study could not confirm these findings (studies with negative outcome; NO studies). Differences in study design may again explain this discrepancy (details are provided in Table 5). First, the PO studies had on average smaller sample sizes than the NO studies (PO studies: 9.3 schizophrenic patients versus 7.7 controls; NO studies: 21.2 versus 25.4 ); second, except in the study by Dorph-Petersen et al. (2004), in all NO studies, both left and right MDNTs were investigated, whereas in the PO studies, only either the left or the right MDNTs were analysed, and third, the average age of the schizophrenic patients and controls was higher in the PO studies than in the NO studies [PO studies: 65 years (schizophrenic patients)] versus 65 years (controls); NO studies: 49 versus 51 years). However, small sample sizes and additional, confounding pathologies (in this case possible age-related neurodegeneration) have been identified as major potential shortcomings in most studies addressing the neuropathology of schizophrenia (Harrison, 1999).

Meta-analyses of clinical neuroimaging studies found reductions in the mean volume of the amygdala in schizophrenic patients compared with controls in the range of 5-10\% (Lawrie and Abukmeil, 1998; Nelson et al., 1998; Wright et al., 2000; see also Honea et al., 2005). Although these clinical neuroimaging studies did not differentiate between the nuclei of the amygdala, these data are in line with the results of the present study as well as with results from a post-mortem study by Bogerts (1984) who found a significantly re- 
duced volume of the amygdala in schizophrenic patients compared with controls (-22\%). In contrast, Heckers et al. (1990) and Chance et al. (2002) did not observe volume reductions of the amygdala in schizophrenia in post-mortem studies; the reason for this discrepancy is not known. Nevertheless, Chance et al. (2002) suggested that there may be alterations in other morphological aspects of the amygdala such as cellular composition, as found in the present study.

It should be mentioned that several reports have suggested schizophrenia being associated with a disturbance of cerebral asymmetry (for review see Crow, 1990, 1997; Petty, 1999). This did not apply to the findings of the present study in which no significant differences between the left and the right hemispheres were found for the schizophrenic patients and the controls. This is in agreement with other studies of subcortical pathology in schizophrenia. For instance, a meta-analysis of clinical neuroimaging studies, in which a volume of $100 \%$ in the comparison group was assumed, found for patients with schizophrenia an overall volume of $94 \%$ in the left and right amygdala (Wright et al., 2000). Disturbance of cerebral asymmetry in schizophrenia might therefore be restricted to discrete cortical regions such as the dorsolateral prefrontal cortex (Cullen et al., 2006).

What then, in this context, does the pattern of rather subtle neuropathological alterations reported in the present study contribute to our understanding of the pathogenesis of schizophrenia? Generally, two non-exclusive hypotheses for the development of schizophrenia have been proposed, a neurodevelopmental one (Lieberman, 1999; Tsuang, 2000; Lewis and Levitt, 2002; Murray et al., 2004) and a neurodegenerative one (Ashe et al., 2001; Sawa and Snyder, 2002; Church et al., 2002). A neurodevelopmental origin for schizophrenia has become the prevailing pathogenic hypothesis in recent decades (Weinberger, 1987; Lewis and Lewitt, 2002; Murray et al., 2004). Specifically, genetic and nongenetic mechanisms are thought to interact, in as yet not understood ways, to affect the developing brain, resulting in a predisposition to schizophrenia (see, e.g. Schmidt-Kastner et al., 2006). Although several genes have been proposed as candidates for schizophrenia (for review see Davis et al., 2003; Harrison and Owen, 2003; Owen et al., 2004; Harrison and Weinberger, 2005; Kirov et al., 2005), genetic defects may not account for all aspects of the pathogenesis of schizophrenia (Kendler, 2005). There is no direct evidence for a neurodevelopmental or a neurodegenerative origin of the results of the present study. Yet, considering that the reductions in both volumes of brain regions (CGM, putamen, LNA) and total neuron numbers ( $\mathrm{CN}$, putamen and LNA) did not correlate with illness duration, it is tempting to speculate that such negative correlation is indicative of a neurodevelopmental deficit. Particularly in respect to the alterations found in the LNA, it is noteworthy that early postnatal lesions of the amygdala in rats lead to a combination of behavioural changes which share features with symptoms of schizophrenia, whereas adult amygdala lesions do not produce such changes (Wolterink et al., 2001). Furthermore, tract-tracing studies have shown that connections between the basolateral amygdala and the prefrontal cortex (including the anterior cingulate cortex) mature slowly during postnatal develop- ment in rats (Bouwmeester et al., 2002; Cunningham et al., 2002). A slow maturation of connections 
may also be important for the primate brain, because lesions of mesial temporal limbic structures, including the amygdala, in postnatal monkeys (but not in mature monkeys) are associated with abnormal function of the prefrontal cortex of adult animals (Bertolino et al., 1997). Accordingly, alterations in the prolonged maturation of connections between the amygdala and the prefrontal cortex have been hypothesized to be involved during the onset of schizo- phrenia in adolescence (Benes, 2003). Reduced total neuron numbers in the LNA based on a neurodevelopmental deficit could play an important role in these pathological processes and deserves further investigation.

Deficiencies in limbic functions which many researchers believe are the cause of the productive symptoms of schizophrenia such as paranoia and psychosis (Alexander et al., 1990; Bogerts, 1997; Weinberger, 1997) must have another neuropathological basis than reductions in the total neuron number in the NA [as suggested by Thune and Pakkenberg (2000)1. Likewise, involvement of MDNT in positive symptoms of schizophrenia [as discussed by, e.g. Andreasen et al. (1994), Friston (1999) and Stephan et al. (2006)1 must have another neuropathological basis than reductions in the total neuron number in this nucleus [as suggested by, e.g. Thune and Pakkenberg (2000)1. Abnormal anatomical connections (i.e. 'miswiring' of asso- ciation fibres), impairments in synaptic transmission and plasticity or complex combinations of both are the most relevant candidates in this regard (Stephan et al., 2006).

Furthermore, the hyperdopaminergic state of the striatum is one of the most important aspects of the pathophysiology and treatment of schizophrenia, but the mechanisms leading to increased dopamine levels in the basal ganglia are more complex than initially thought (Abi-Dargham et al., 2000; Carlsson et al., 2001; Winterer and Weinberger, 2004). The small decrease in neurons found in the present study could be due to a prolonged neurodevelopmental perturbation affecting several processes in the striatum, including neurogenesis and the innervation by dopaminergic mesencephalic fibres. A simple mismatch between neuronal numbers and dopaminergic innervation, however, is unlikely to explain long-lasting effects on neurotransmission, because glutamatergic cortical inputs and transmitters of striatal interneurons also come into play. Finally, the reduced number of neurons in LNA found in the present study could be involved in emotional disturbance in schizophrenia. According to Yaniv et al. (2004), LNA is a secondary interface limited to relatively simple, unimodal conditioned stimulus features, whereas BNA serves as an amygdalar sensory interface for complex, configural stimulus information. On the other hand, at least in rat, LNA is reciprocally connected with both BNA and ABNA (Savander et al., 1997). Accordingly, a reduced number of neurons in the LNA could result in impaired function of BNA in schizophrenia, with potential influence on at least two functional systems. First, the BNA interacts with striatal and cortical motor circuits, allowing active response to emotional arousing stimuli which is impaired in schizophrenia (Aleman and Kahn, 2005). Second, impaired output of the basolateral amygdala (including LNA and BNA) to the central nucleus of the amygdala (CNA) could disturb information flow from the CNA to brainstem areas involved in controlling specific involuntary components of emotional reac- 
tion (such as autonomic and endocrine responses) that are also disturbed in schizophrenia (Aleman and Kahn, 2005). Notably, the reduced number of neurons in the LNA could even be involved in alterations of the dopaminergic system in schizophrenia. This is due to the fact that all subdivisions of the amygdale project to the ventral striatum and therefore can indirectly influence dopaminergic neurons through amygdalo-striato-nigral loops (Haber and Fudge, 1997; Fudge et al., 2002; Fudge and Emiliano, 2003).

In summary, the results of the present study support a neurodevelopmental deficit in certain subcortical regions in schizophrenia, primarily affecting the development of the basolateral amygdala and the dorsal striatum. Further studies are required to address other neuropatho- logical alterations in the prefrontal cortex and the dopaminergic system in schizophrenia and their possible interactions with disturbances of the development of the basolateral amygdala and dorsal striatum.

\section{Supplementary material}

Supplementary material is available at Brain Online.

\section{Acknowledgements}

We thank A. Broschk for expert technical assistance. This work was supported by Stanley Medical Research Institute (\#02R-258, \#04R-674 to H.H., P.R.H. and C.S.), the European Community (Quality of Life and Management of Living Resources, QLK6-CT-2000-60042, QLK6-GH-00-60042-46, to P.K.) and NIH grant MH66392 to P.R.H.

\section{References}

Abi-Dargham A, Rodenhiser J, Printz D, Zea-Ponce Y, Gil R, Kegeles LS, et al. Increased baseline occupancy of D2 receptors by dopamine in schizophrenia. Proc Natl Acad Sci USA 2000; 97: 8104-9

Aleman A, Kahn RS. Strange feelings: do amygdala abnormalities dysregulate the emotional brain in schizophrenia? Prog Neurobiol 2005; 77: 283-98

Alexander GE, Crutcher MD, DeLong MR. Basal ganglia-thalamocortical circuits: parallel substrates for motor, oculomotor, "prefrontal" and "limbic" functions. Prog Brain Res 1990; 85: 119-46

Andreasen NC, Arndt S, Swayze V II, Cizadlo T, Flaum M, O'Leary D, et al. Thalamic abnormalities in schizophrenia visualized through magnetic resonance image averaging. Science 1994; 266: 294-8

Andreasen NC, Nopoulos P, O'Leary DS, Miller DD, Wassink T, Flaum M. Defining the phenotype of schizophrenia: cognitive dysmetria and its neural mechanisms. Biol Psychiatry 1999; 46: 908-20 
Ashe PC, Berry MD, Boulton AA. Schizophrenia, a neurodegenerative disorder with neurodevelopmental antecedents. Prog Neuropsychopharmacol Biol Psychiatry 2001; 25: 691-707

Beckmann H, Lauer M. The human striatum in schizophrenia. II. Increased number of striatal neurons in schizophrenics. Psychiatry Res 1997; 68: 99-109

Benes FM. Schizophrenia, II: amygdalar fiber alteration as etiology? Am J Psychiatry 2003; 160: 1053

Bertolino A, Saunders RC, Mattay VS, Bachevalier J, Frank JA, Weinberger DR. Altered development of prefroncal neurons in rhesus monkeys with neonatal mesial temporo-limbic lesions: a proton magnetic resonance spectroscopic imaging study. Cereb Cortex 1997; 7: 740-8

Bogerts B. Zur Neuropathologie der Schizophrenien. Fortschr Neurol Psychiatr 1984; 52: 428-37

Bogerts B. The temporolimbic systern theory of positive schizophrenic symptoms. Schizophr Bull 1997; 23: 423-35

Bouwmeester $\mathrm{H}$, Wolterink $\mathrm{G}$, van Ree JM. Neonatal development of projections from the basolateral amygdala to prefrontal, striatal, and thalamic structures in the rat. J Comp Neurol 2002; 442: 239-49

Braak H, Braak E. Neuronal types in the basolateral amygdaloid nuclei of man. Brain Res Bull 1983; 11:349-65

Braak H, Braak E. Staging of Alzheimer's disease-related neurofibrillary changes. Neurobiol Aging 1995; 16: 271-8 Brockhaus H. Zur feineren Anatomie des Septum und des Striatum. J Psychol Neurol 1942; 5: 1-56

Byne W, Buchsbaum MS, Mattiace LA, Hazlett EA, Kemether E, Elhakem SL, et al. Postmortem assessment of thalamic nuclear volumes in subjects with schizophrenia. Am J Psychiatry 2002; 159: 59-65

Carlsson A, Waters N, Holm-Waters S, Tedroff J, Nilsson M, Carlsson ML. Interactions between monoamines, glutamate, and GABA in schizophrenia: new evidence. Annu Rev Pharmacol Toxicol 2001; 41; 237-60

Casanova MF, de Zeeuw L, Switala A, Kreczmanski P, Korr H, Ulfig N, et al. Mean cell spacing abnormalities in the neocortex of patients with schizophrenia. Psychiatry Res 2005; 133: 1-12

Cavalieri B. Geomerria indivisibilibus continuorum. Typis Clementis Ferronij, Bononiae, 1635 (reprinted 1966 as Geometria Degli Indivisibili. Unione Tipografico-Editrice Torinese, Torino)

Chakos MH, Lieberman JA, Bilder RM, Borenstein M, Lerner G, Bogerts B, et al. Increase in caudate nuclei volumes of first-episode schizophrenic patients taking antipsychotic drugs. Am J Psychiatry 1994; 151: 1430-6

Chance SA, Esiri mm, Crow TJ. Amygdala volume in schizophrenia: post-mortem study and review of magnetic resonance imaging findings. Br J Psychiatry 2002; 180: 331-8

Church SM, Cotter D, Bramon E, Murray RM. Does schizophrenia result from developmental or degenerative processes? J Neural Transm Suppl 2002; 63: 129-47

Crow TJ. Temporal lobe asymmetries as the key to the etiology of schizophrenia. Schizophren Bull 1990;16:433-43

Crow TJ. Schizophrenia as failure of hemispheric dominance for language. Trends Neurosci 1997; 20: 339-43

Cullen TJ, Walker MA, Parkinson N, Craven R, Crow TJ, Esiri mm, et al. A postmortem study of the mediodorsal nucleus of the thalamus in schizophrenia. Schizophr Res 2003; 60: 157-66

Cullen TJ, Walker MA, Eastwood SL, Esiri mm, Harrison PJ, Crow TJ. Anomalies of asymmetry of pyramidal cell density and structure in dorsolateral prefrontal cortex in schizophrenia. Br J Psychiarry 2006; 188: 26-31

Cunningham MG, Bhattacharyya S, Benes FM. Amygdalo-cortical sprout- ing continues into early adulthood: implications for the development of normal and abnormal function during adolescence. J Comp Neurol 2002; 453: 116-30

Danos P, Schmidt A, Baumann B, Bernstein HG, Northoff G, Stauch R, et al. Volume and neuron number of the mediodorsal thalamic nucleus in schizophrenia: a replication study. Psychiatry Res 2005; 140: 281-9

Davis KL, Stewart DG, Friedman JI, Buchsbaum M, Harvey PD, Hof PR, et al. White matter changes in schizophrenia: evidence for myelin- related dysfunction. Arch Gen Psychiatry 2003; 60: 443-56 
Chapter 2

Dewulf A. Anatomy of the normal human thalamus: topomerry and standardized nomenclature. Amsterdam: Elsevier; 1971

Dorph-Petersen KA, Pierri JN, Sun Z, Sampson AR, Lewis DA.Stereological analysis of the mediodorsal thalamic nucleus in schizophrenia: volume, neuron number, and cell types. J Comp Neurol 2004; 472: 449-62

Frazier JA, Giedd JN, Kaysen D, Albus K, Hamburger S, Alaghband-Rad J, et al. Childhood-onset schizophrenia: brain MRI rescan after 2 years of clozapine maintenance treatment. Am J Psychiatry 1996; 153: 564-6.

Friston KJ. Schizophrenia and the disconnection hypothesis. Acta Psychiatr Scand Suppl 1999; 395: 68-79

Fudge JL, Emiliano AB. The extended amygdala and the dopamine system: another piece of the dopamine puzzle. J Neuropsychiatry Clin Neurosci 2003; 15:301-16

Fudge JL, Kunishio K, Walsh P, Richard C, Haber SN. Amygdaloid projections to ventromedial striatal subterritories in the primate. Neuroscience 2002; 110:257-75

Gundersen HJG, Jensen EB. The efficiency of systematic sampling and its prediction. J Microsc 1987; 147: 229-63

Haber SN, Fudge JL. The interface between dopamine neurons and the amygdala: implications for schizophrenia. Schizophr Bull 1997; 23: 471-82

Harrison PJ. The neuropathology of schizophrenia. A critical review of the data and their interpretation. Brain 1999; 122: 593-624

Harrison PJ, Owen MJ. Genes for schizophrenia? Recent findings and their pathophysiological implications. Lancet 2003; 361: 417-9

Harrison PJ, Weinberger DR. Schizophrenia genes, gene expression, and neuropathology: on the matter of their convergence. Mol Psychiatry 2005; 10: 40-68

Heckers S, Heinsen H, Heinsen YC, Beckmann H. Limbic structures and lateral ventricle in schizophrenia. A quantitative postmortem study. Arch Gen Psychiatry 1990; 47: 1016-22

Heinsen $\mathrm{H}$, Heinsen YL. Serial thick, frozen, gallocyanin stained sections of human central nervous system. J Histotechnol 1991; 14: 167-73

Heinsen H, Beckmann H, Heinsen YL, Gallyas F, Haas S, Scharff G. Laminar neuropathology in Alzheimer's disease by a modified Gallyas impregnation. Psychiatry Res 1989; 29:463-5

Heinsen H, Henn R, Eisenmenger W, Gotz M, Bohl J, Bethke B, et al. Quantitative investigations on the human entorhinal area: left - right asymmetry and age-related changes. Anat Embryol 1994; 190: $181-94$

Heinsen H, Rue b U, Bauer M, Ulmar G, Bethke B, Schu" ler M, et al. Nerve cell loss in the thalamic mediodorsal nucleus in Huntington's disease. Acta Neuropathol 1999; 97: 613-22

Heinsen $\mathrm{H}$, Arzberger T, Schmitz C. Celloidin mounting (embedding without infiltration) - a new, simple and reliable method for producing serial sections of high thickness through complete human brains and its application to stereological and immunohistochemical investigations. J Chem Neuroanat 2000; 20:49-59

Hirai T, Jones EG. A new parcellation of the human thalamus on the basis of histochemical staining. Brain Res Rev 1989; 14: 1-34

Holt DJ, Herman mm, Hyde TM, Kleinman JE, Sinton CM, German DC, et al. Evidence for a deficit in cholinergic interneurons in the striatum in schizophrenia. Neuroscience 1999; 94: 21-31

Honea R, Crow TJ, Passingham D, Mackay CE. Regional deficits in brain volume in schizophrenia: a metaanalysis of voxel-based morphometry studies. Am J Psychiarry 2005; 162: 2233-45

Jones EG. A description of the human thalamus. In: Steriade M, Jones EG, McCormick DA, editors. Experimental and clinical aspects. Thalamus, Vol. II. Oxford: Elsevier Science; 1997. p. 425-500 
Kasai K, Iwanami A, Yamasue H, Kuroki N, Nakagome K, Fukuda M. Neuroanatomy and neurophysiology in schizophrenia. Neurosci Res 2002; 43: 93-110

Kemether EM, Buchsbaum MS, Byne W, Hazlett EA, Haznedar M, Brickman AM, et al. Magnetic resonance imaging of mediodorsal, pulvinar, and centromedian nuclei of the thalamus in patients with schizophrenia. Arch Gen Psychiatry 2003; 60: 983-91

Kendler KS. "A gene for ...." The nature of gene action in psychiatric disorders. Am J Psychiatr 2005; 162: 1243-52

Kirov G, O'Donovan MC, Owen MJ. Finding schizophrenia genes. J Clin Invest 2005; 115: 1440-8

Kreczmanski P, Schmidt-Kastner R, Heinsen H, Steinbusch HW, Hof PR, Schmitz C. Stereological studies of capillary length density in the frontal cortex of schizophrenics. Acta Neuropathol 2005; 109: 510-8

Lang DJ, Kopala LC, Vandorpe RA, Rui Q, Smith GN, Goghari VM, et al. Reduced basal ganglia volumes after switching to olanzapine in chronically treated patients with schizophrenia. Am J Psychiatry 2004; 161: 1829-36

Lauer M, Heinsen H. Cytoarchitectonics of the human nucleus accumbens. J Hirnforsch 1996; 37: 243-54

Lauer $\mathrm{M}$, Beckmann $\mathrm{H}$. The human striatum in schizophrenia. 1. Increase in overall relative striatal volume in schizophrenics. Psychiatry Res 1997; 68: 87-98

Lauer M, Senitz D, Beckmann H. Increased volume of the nucleus accumbens in schizophrenia. J Neural Transm 2001; 108: 645-60

Lawrie SM, Abukmeil SS. Brain abnormality in schizophrenia. A systematic and quantitative revicw of volumetric magnetic resonance imaging studies. Br J Psychiatry 1998; 172: 110-20

Lewis DA, Levitt P. Schizophrenia as a disorder of neurodevelopment. Annu Rev Neurosci 2002; 25: 409-32

Lieberman JA. Is schizophrenia a neurodegenerative disorder? A clinical and neurobiological perspective. Biol Psychiatry 1999; 46: 729-39

McCarley RW, Wible CG, Frumin M, Hirayasu Y, Levitt JJ, Fischer IA, et al. MRI anatomy of schizophrenia. Biol Psychiatry 1999; 45: 1099-119

Murray RM, Sham P, Van Os J, Zanelli J, Cannon M, McDonald C. A developmental model for similarities and dissimilarities between schizophrenia and bipolar disorder. Schizophrenia Res 2004; 71: 405-16.

Nelson MD, Saykin AJ, Flashman LA, Riordan HJ. Hippocampal volume reduction in schizophrenia as assesscd by magnetic resonance imaging; a meta-analytic study. Arch Gen Psychiatry 1998; 55: 433-40

Owen MJ, Williams NM, O'Donovan MC. The molecular genetics of schizophrenia: new findings promise new insights. Mol Psychiatry 2004; 9: 14-27

Pakkenberg B. Pronounced reduction of total neuron number in mediodorsal thalamic nucleus and nucleus accumbens in schizophrenics. Arch Gen Psychiatry 1990; 47: 1023-8

Popken GJ, Bunney WE, Potkin SG, Jones EG. Subnucleus-specific loss of neurons in medial thalamus of schizophrenics. Proc Natl Acad Sci USA 2000; 97: 9276-80

Petty RG. Structural asymmetries of the human brain and their disturbance in schizophrenia. Schizophren Bull 1999; 25: 121-39

Savander V, Miettinen R, Ledoux JE, Pitkanen A. Lateral nucleus of the rat amygdala is reciprocally connected with basal and accessory basal nuclei: a light and electron microscopic study. Neuroscience 1997; 77: 767-81

Sawa A, Snyder SH. Schizophrenia: diverse approaches to a complex disease. Science 2002; 296: 692-5

Schmidt-Kastner R, van Os J, Steinbusch HWM, Schmitz C. Gene regulacion by hypoxia and the neurodevelopmental origin of schizo- phrenia. Schizophr Res 2006; 84: 253-71 
Schmitz C. Variation of fractionator estimates and its prediction. Anat Embryol 1998; 198: 371-97

Schmitz C, Hof PR. Recommendations for straightforward and rigorous methods of counting neurons based on a computer simulation approach. J Chem Neuroanat 2000; 20:93-114

Schmitz C, Hof PR. Design-based stereology in neuroscience. Neuroscience 2005; 130: 813-31

Schmitz C, Grolms N, Hof PR, Boehringer R, Glaser J, Korr H. Altered spatial arrangement of layer V pyramidal cells in the mouse brain following prenatal low-dose X-irradiation. A stereological study using a novel three-dimensional analysis method to estimate the nearest neighbor distance distributions of cells in thick sections. Cereb Cortex 2002; 12: 954-60

Stereological estimation of the number of neurons in the human anygdaloid complex. J Comp Neurol 2005; 491: 320-9, 2006; 494: 704. Schumann CM, Amaral DG. Stereological analysis of amygdala neuron number in autism. J Neurosci 2006; 26: 7674-9

Selemon LD. Regionally diverse cortical pathology in schizophrenia: clues to the etiology of the disease. Schizophren Bull 2001; 27: 349-77

Shenton ME, Dickey CC, Frumin M, McCarley RW. A review of MRI findings in schizophrenia. Schizophren Res 2001; 49: 1-52

Sims KS, Williams RS. The human amygdaloid complex: a cytologic and histochemical atlas using Nissl, myelin, acetylcholinesterase and nicotinamide adenine dinucleotide phosphate diaphorase staining. Neuroscience 1990; 36: 449-72

Sorvari $\mathrm{H}$, Soininen H, Pitkanen A. Calbindin-D28K-immunoreactive cells and fibres in the human amygdaloid complex. Neuroscience 1996; 75:421-43

Stephan KE, Baldeweg T, Friston KJ. Synaptic plasticity and dysconnection in schizophrenia. Biol Psychiatry 2006; 59: 929-39

Tamminga CA, Vogel M, Gao X, Lahti AC, Holcomb HH. The limbic cortex in schizophrenia: focus on the anterior cingulate. Brain Res Rev 2000; 31:364-70

Thune JJ, Pakkenberg B. Stereological studies of the schizophrenic brain. Brain Res Rev 2000; 31: 200-4

Tsuang MT. Schizophrenia: genes and environment. Biol Psychiatry 2000; 47: 210-20

Weinberger DR. Implications of normal brain development for the pathogenesis of schizophrenia. Arch Gen Psychiatry 1987; 44: 660-9

Weinberger DR. The biological basis of schizophrenia: new directions. J Clin Psychiatry 1997; 58 Suppl 10: 22-7

West MJ, Slomianka L, Gundersen HJ. Unbiased stereological estimation of the total number of neurons in the subdivisions of the rat hippocampus using the optical fractionator. Anat Rec 1991; 231: 482-97

Winterer G, Weinberger DR. Genes, dopamine and cortical signal-to-noise ratio in schizophrenia. Trends Neurosci 2004; 27: 683-90

Wolterink G, Daenen LE, Dubbeldam S, Gerrits MA, van Rijn R, Kruse CG, et al. Early amygdala damage in the rat as a model for neurodevelop- mental psychopathological disorders. Eur Neuropsychopharmacol 2001; 11:51-9

Wright IC, Rabe-Hesketh S, Woodruff PW, David AS, Murray RM, Bullmore ET. Meta-analysis of regional brain volumes in schizophrenia. Am J Psychiatry 2000;157:16-25

Yaniv D, Desmedt A, Jaffard R, Richter-Levin G. The amygdala and appraisal processes: stimulus and response complexity as an organizing factor. Brain Res Rev 2004; 44: 179-86

Young KA, Manaye KF, Liang C, Hicks PB, German DC. Reduced number of mediodorsal and anterior thalamic neurons in schizophrenia. Biol Psychiatry 2000; 47: 944-53 
Zipursky RB, Lim KO, Sullivan EV, Brown BW, Pfefferbaum A. Widespread cerebral gray matter volume deficits in schizophrenia. Arch Gen Psychiatry 1992; 49: 195-205

Zipursky RB, Seeman MV, Bury A, Langevin R, Wortzman G, Katz R. Deficits in gray matter volume are present in schizophrenia but nor bipolar disorder. Schizophr Res 1997; 26: 85-92 


\section{Tables}

Table 1. Clinical characteristics of all cases included in this study

\begin{tabular}{ccclrrrr} 
No & A & O & \multicolumn{1}{c}{ Cause of death } & PMI & \multicolumn{1}{c}{ Fix } & \multicolumn{2}{c}{ Diagnosis } \\
\hline & $(\mathrm{y})$ & $(\mathrm{y})$ & & $(\mathrm{h})$ & \multicolumn{1}{c}{ (d) } & DSM-IV & ICD-10 \\
S1 & 22 & 19 & Suicidea & 88 & 130 & 295.30 & F20.00 \\
S2 & 36 & 28 & Suicidea & $<72$ & 115 & 295.30 & F20.00 \\
S3 & 46 & 24 & Systemic hypothermia & $<24$ & 327 & 295.30 & F20.01 \\
S4 & 50 & 17 & Peritonitis & $<24$ & 203 & 295.30 & F20.00 \\
S5 & 50 & 22 & Suicide & 18 & 170 & 295.30 & F20.00 \\
S6 & 51 & 17 & Septicaemia & 33 & 127 & 295.60 & F20.50 \\
S7 & 54 & 20 & Septicaemia & 27 & 250 & 295.60 & F20.50 \\
S8 & 55 & 22 & Right-sided heart failure & 25 & 84 & 295.30 & F20.00 \\
S9 & 57 & 37 & Septicaemia & 76 & 163 & 295.30 & F20.00 \\
S10 & 60 & 24 & Pulmonary embolism & $<48$ & 311 & 295.30 & F20.01 \\
S11 & 62 & 19 & Aspiration & 7 & 171 & 295.30 & F20.00 \\
S12 & 63 & 22 & Acute myocardial infarct & 15 & 338 & 295.60 & F20.50 \\
S13 & 64 & 26 & Pulmonary embolism & 6 & 817 & 295.60 & F20.50 \\
C1 & 25 & - & Cardiac tamponade & 14 & 119 & - & - \\
C2 & 36 & - & Gunshot & 24 & 143 & - & - \\
C3 & 47 & - & Acute myocardial infarct & $<24$ & 133 & - & - \\
C4 & 50 & - & Acute myocardial infarct & 35 & 433 & - & - \\
C5 & 50 & - & Avalanche accident & 23 & 498 & - & - \\
C6 & 51 & - & Septicaemia & 7 & 285 & - & - \\
C7 & 54 & - & Acute myocardial infarct & 18 & 168 & - & - \\
C8 & 56 & - & Acute myocardial infarct & 60 & 3570 & - & - \\
C9 & 58 & - & Acute myocardial infarct & 28 & 126 & - & - \\
C10 & 60 & - & Gastrointestinal haemorrhage & 18 & 101 & - & - \\
C11 & 60 & - & Gastrointestinal haemorrhage & 27 & 302 & - & - \\
C12 & 62 & - & Acute myocardial infarct & $<24$ & 3696 & - & - \\
C13 & 65 & - & Bronchopneumonia & 6 & 2289 & - & - \\
\hline
\end{tabular}

$S=$ schizophrenic patient; $C=$ control; $A=$ age at death; $O=$ age at onset; $P M I=$ post-mortem interval (time between death and autopsy); Fix $=$ fixation time; $y=$ years; $h=$ hours; $d=$ days. aThese two patients had relatively long post-mortem intervals. However, both had committed suicide (one by hanging, the other by jumping from a building), were found within 1 h of death and were kept at $4^{\circ} \mathrm{C}$ until autopsy. Accordingly, the post-mortem intervals between death and autopsy of these patients cannot be compared with the corresponding intervals of the other cases and were thus excluded from the calculation of the mean post-mortem intervals. ${ }^{\text {tTT }}$ the volume of the CGM of these cases could not be analysed because sections from the frontal as well as the occipital poles were missing. 
Table 2. Details of the stereological counting procedures

\begin{tabular}{cccccc} 
& CN & P & NA & MDNT & LNA \\
\hline Obj. & 40 & 40 & 40 & 20 & 40 \\
$\mathrm{~B}(\mu \mathrm{m} 2)$ & 10000 & 10000 & 3600 & 15298 & 3600 \\
$\mathrm{H}(\mathrm{mm})$ & 30 & 30 & 20 & 15 & 10 \\
$\mathrm{D}(\mathrm{mm})$ & 3000 & 3000 & 600 & 800 & 600 \\
$\Sigma \mathrm{OD}$ & 171 & 196 & 461 & 626 & 767 \\
$\Sigma \mathrm{Q}$ & 755 & 761 & 499 & 1071 & 1110 \\
CE pred $(\mathrm{n})$ & 0.036 & 0.036 & 0.048 & 0.031 & 0.031 \\
\hline
\end{tabular}

$C N=$ caudate nucleus; $P=$ putamen; NA = nucleus accumbens; MDNT = mediodorsal nucleus of the thalamus; $L N A=$ lateral nucleus of the amygdala; Obj. = objective used; $B$ and $H=$ base and height of the unbiased virtual counting spaces; $\mathrm{D}=$ distance between the unbiased virtual counting spaces in mutually ortho-gonal directions $x$ and $y ; \Sigma O D=$ average sum of unbiased virtual counting spaces used; $\Sigma Q=$ average number of counted neurons; CEpred $(n)=$ average predicted coefficient of error of the estimated total numbers of neurons using the prediction method described by Schmitz (1998) and Schmitz and Hof (2000).]

Table 3. Results of statistical analysis (P-values) with generalized linear model MANOVA

\begin{tabular}{cccccccc} 
Variable & $\begin{array}{c}\text { Brain } \\
\text { region }\end{array}$ & A/ID & PMI & Fix & D & H & D x H \\
\hline Volume & CGM & 0.299 & 0.609 & 0.223 & 0.008 & 0.392 & 0.601 \\
& CN & 0.143 & 0.973 & 0.961 & 0.209 & 0.436 & 0.860 \\
& P & 0.082 & 0.004 & 0.169 & 0.020 & 0.782 & 0.929 \\
& NA & 0.600 & 0.136 & 0.572 & 0.299 & 0.394 & 0.842 \\
& MDNT & 0.581 & 0.125 & 0.574 & 0.161 & 0.225 & 0.546 \\
& BNA & 0.335 & 0.851 & 0.422 & 0.042 & 0.404 & 0.926 \\
Neuron density & ABNA & 0.515 & 0.463 & 0.416 & 0.858 & 0.053 & 0.497 \\
& LNA & 0.900 & 0.628 & 0.462 & 0.006 & 0.988 & 0.520 \\
& CN & 0.916 & 0.617 & 0.727 & 0.866 & 0.627 & 0.676 \\
& P & 0.929 & 0.893 & 0.834 & 0.393 & 0.897 & 0.559 \\
& NA & 0.054 & 0.204 & 0.118 & 0.735 & 0.209 & 0.955 \\
& MDNT & 0.442 & 0.188 & 0.800 & 0.105 & 0.781 & 0.987 \\
& LNA & 0.126 & 0.892 & 0.507 & 0.786 & 0.835 & 0.389 \\
& CN & 0.047 & 0.861 & 0.297 & 0.011 & 0.764 & 0.932 \\
& P & 0.062 & 0.012 & 0.129 & 0.005 & 0.792 & 0.707 \\
& NA & 0.046 & 0.856 & 0.089 & 0.200 & 0.893 & 0.933 \\
& MDNT & 0.818 & 0.555 & 0.975 & 0.419 & 0.529 & 0.698 \\
& LNA & 0.170 & 0.608 & 0.898 & 0.015 & 0.591 & 0.926 \\
\hline
\end{tabular}

$C G M=$ cortical grey matter; $C N=$ caudate nucleus; $P=$ putamen; $N A=$ nucleus accumbens; $M D N T=$ mediodorsal nucleus of the thalamus; $B N A=$ basal nucleus of the amygdala; $A B N A=$ accessory basal nucleus of the amygdala; $L N A=$ lateral nucleus of the amygdala; $A / D=$ age (controls) or adjusted illness duration (schizophrenic patients), respectively (adjusted illness duration calculated as age at death minus age at onset plus mean age at onset; details are provided in Statistical analysis); PMI = post-mortem interval (time between death and autopsy); Fix = fixation time; $\mathrm{D}=$ diagnosis; $\mathrm{H}=$ hemisphere. P-values smaller than 0.05 are shown in boldface. 
Table 4. Reports in the literature of estimated mean volumes of and estimated total neuron numbers in four subcortical regions in the brains of controls and schizophrenic patients (averaged for left and right hemispheres)

\begin{tabular}{|c|c|c|c|c|}
\hline Source & $\mathrm{CN}$ & $\mathbf{P}$ & NA & LNA \\
\hline \multicolumn{5}{|c|}{ Volume data for all control casus (all values in $\mathrm{mm}^{3}$ ) } \\
\hline Present study & 2212 & 2713 & 204 & 414 \\
\hline 1 & $2043 a$ & $2681 a$ & $522 a$ & \\
\hline 2 & $2565 b, c$ & $2979 b$ & & \\
\hline 3 & & & $147 d$ & \\
\hline 4 & & & 138 & \\
\hline 5 & & & & 452 \\
\hline \multicolumn{5}{|c|}{ Volume data for schizophrenic patients (compared with controls) } \\
\hline Present study & $-9,1 \%(n s)$ & $-4,6 \%(n 5)^{*}$ & $-8,8 \%$ (ns) & $-15,0 \% *$ \\
\hline 1 & $-4,5 \%$ (ns) & $-4,1 \%(n s)$ & $+1,2 \%$ (ns) & \\
\hline 2 & $-0.3 \%(n s)$ & $+1.9 \%$ (ns) & & \\
\hline 3 & & & $+10.5 \%$ (ns) & \\
\hline 4 & & & $-42.0 \% *$ & \\
\hline \multicolumn{5}{|c|}{ Total neuron number data for control cases (all values in millions) } \\
\hline Present study & 32,7 & 35,4 & 2,43 & 4,43 \\
\hline 4 & $28,2 \mathrm{e}$ & 30,0 & & \\
\hline 5 & & & 2,93 & \\
\hline 6 & & & & 4,00 \\
\hline \multicolumn{5}{|c|}{ Total neuron number data for schizophrenic patients compared with controls) } \\
\hline Present study & $-10.2 \% *$ & $-9.9 \% *$ & $-2.1 \%$ (ns) & $-16.0 \%^{*}$ \\
\hline 6 & $+16.6 \%^{*}, f$ & $+9.8 \%(n s)$ & & \\
\hline 4 & & & $-49.5 \% *$ & \\
\hline
\end{tabular}

$C N=$ caudate nucleus; $P=$ putamen; $N A=$ nucleus accumbens; $L N A=$ lateral nucleus of the amygdala. $\mid=B O-$ gerts (1984), a considering an average shrinkage factor of 0.53 reported in this study); 2 = Lauer and Beckmann (1997), b considering an average shrinkage factor of 0.53 reported in this study, $\mathrm{c}$ including the $N A ; 3=$ Lauer et al. (2001), d considering an average shrinkage factor of 0.47 reported in this study; $4=$ Pakkenberg et al. (1990); $5=$ Schumann and Amaral (2006); $6=$ Beckmann and Lauer (1997), e including the NA, $\mathrm{P}<0.05$ only for the right hemisphere (difference: $+17.5 \%$ ) but not for the left hemisphere (difference: $+15.8 \%$ ). ns $=$ not significant (i.e. $P>0.05),{ }^{*} P<0.05$. 
Table 5. Reports in the literature of estimated mean volumes of and estimated total neuron numbers in the MDNT in the brains of controls and schizophrenic patients (averaged for left and right hemispheres)

\begin{tabular}{|c|c|c|c|c|c|c|c|}
\hline Source & $\mathrm{V}\left(\mathrm{mm}^{3}\right)$ & $\mathrm{NN}(\times 106)$ & $\mathrm{n}(\mathrm{S} / \mathrm{C}$ & $\underline{H}$ & $\operatorname{Sex} S(M / F)$ & $C(\mathrm{M} / \mathrm{F})$ & Age $(S / C)$ \\
\hline \multicolumn{8}{|c|}{ Data for controls } \\
\hline 1 & 360 & $\overline{1.81}$ & $12(\mathrm{C})$ & T & & $6 / 6$ & $62(C)$ \\
\hline 2 & 863 & 3.48 & $6(C)$ & I & & $5 / 1$ & $64(C)$ \\
\hline 3 & $701 a$ & $3.17 a$ & $5 a(C)$ & $\mathrm{r}$ & & $4 / 1 a$ & 75 (C) \\
\hline 4 & 680 & 3.88 & $27(C)$ & $1+r$ & & $14 / 13$ & $71(C)$ \\
\hline 5 & $675 b$ & - & $60(C)$ & $1+r$ & & $45 / 15$ & $40(C)$ \\
\hline 6 & 916 & 7.29 & $9(C)$ & 1 & & $6 / 3$ & $54(C)$ \\
\hline 7 & 956 & 6.22 & $18(C)$ & $1+r$ & & $10 / 8$ & $53(C)$ \\
\hline 8 & 569 & 3.79 & $13(\mathrm{C})$ & & & $13 / 0$ & $52(\mathrm{C})$ \\
\hline \multicolumn{8}{|c|}{ Data for schizophrenic patients (compared to controls) } \\
\hline 1 & $-28.9 \%^{*}$ & $-40.3 \% *$ & $12 / 12$ & T & $8 / 4$ & $6 / 6$ & $63 / 62$ \\
\hline 2 & $-17.8 \% *$ & $-27.0 \% *$ & $6 / 6$ & 1 & $5 / 1$ & $5 / 1$ & $66 / 64$ \\
\hline 3 & $-15.0 \% \mathrm{a}_{\prime}^{*}$ & $-29,7 \% \mathrm{a}_{1}^{*}$ & $10 / 5 a$ & $r$ & 4/6a & $4 / 1 a$ & $67 / 75$ \\
\hline 4 & $+4,7 \%$ (ns) & $+5.2 \%$ (ns) & $21 / 27$ & $1+r$ & $11 / 10$ & $14 / 13$ & $68 / 71$ \\
\hline 5 & $-6,7 \%$ (ns) & - & $41 / 60$ & $1+r$ & $32 / 9$ & $45 / 15$ & $37 / 40$ \\
\hline 6 & $+2,1 \%(n s)$ & $-6,5 \%$ (ns) & $11 / 9$ & 1 & $7 / 4$ & $6 / 3$ & $48 / 54$ \\
\hline 7 & $-6,9 \%$ (ns) & $-6,8 \%$ (ns) & $20 / 18$ & $1+r$ & $10 / 10$ & $10 / 8$ & $53 / 53$ \\
\hline 8 & $-8,8 \%$ (ns) & $+4,6 \%$ (ns) & $13 / 13$ & $1+r$ & $13 / 0$ & $13 / 0$ & $52 / 52$ \\
\hline
\end{tabular}

$\mathrm{V}=$ volume; $\mathrm{NN}=$ total neuron number $\mathrm{n}=$ number of analysed brains; $\mathrm{H}=$ hemisphere; $\mathrm{S}=$ schizophrenic patients; $C=$ controls; $M=$ male; $F=$ female; $I=$ left $r=$ right. $1=$ Pakkenberg et al. $(1990) ; 2=$ Popken et al., 2000; 3 = Byne et al. (2002), a without the schizophrenic patients and controls suffering from Alzheimer's disease analysed in this study; $4=$ Cullen et al. (2003); 5 = Kemether et al. (2003), b MRI study; 6 = Dorph-Petersen et al. (2004); $7=$ Danos et al. (2005); $8=$ present study. Ns = not significant (i.e. P40.05), ${ }^{*} P<0.05$. Data of the present study are given boldfaced. 


\section{Figures}

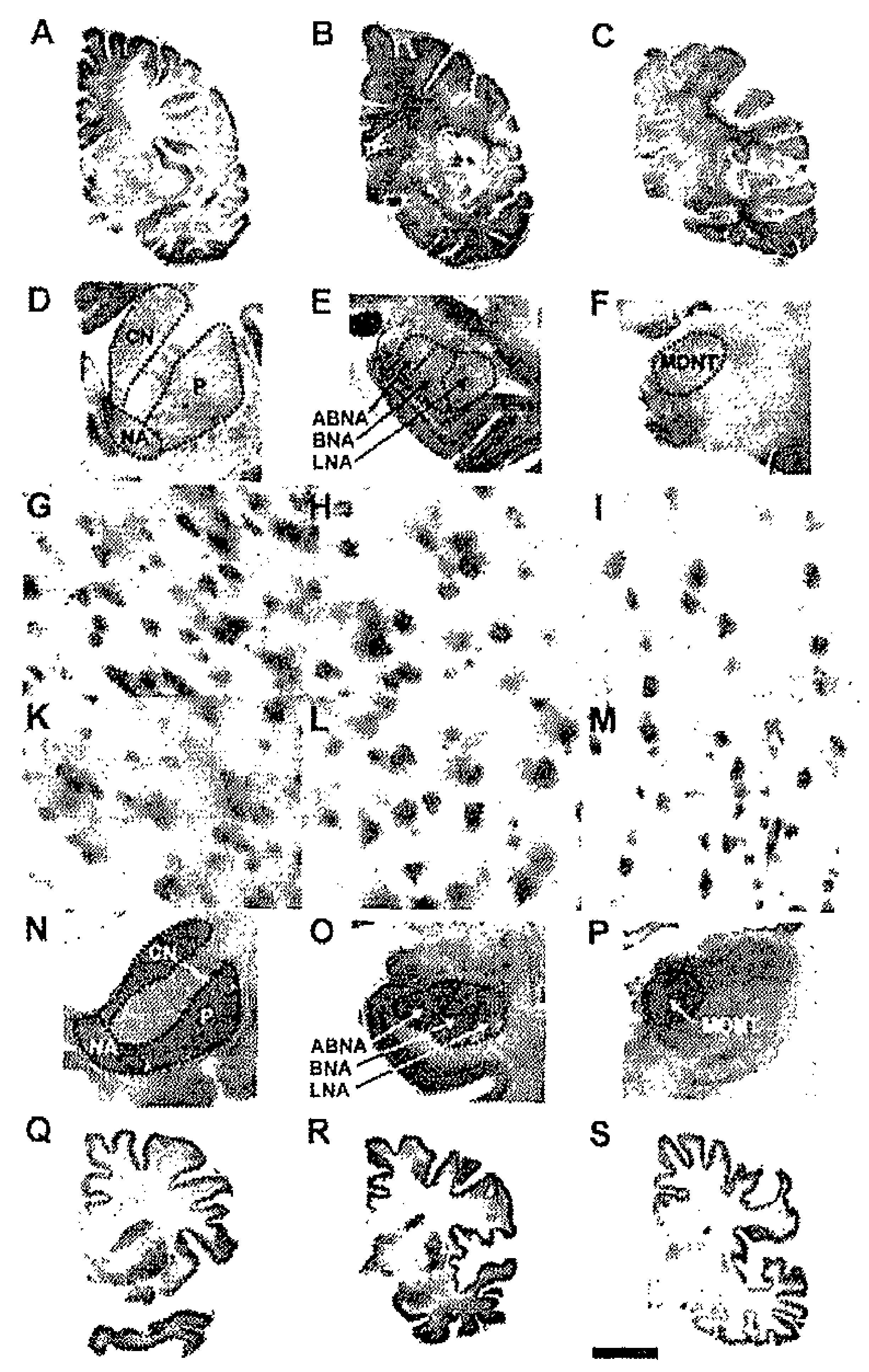

Fig. 1. Photomicrographs of $600 \mathrm{tm}$-thick frontal sections of the right hemisphere from the control CIl (60 years old; $A-1)$ and from the schizophrenic patient $S 13$ (64 years old; $K-S$ ), showing the striatum $(A, D, G, K, N, Q)$, the amygdala $(B, E, H, L, O, R)$ and the thalamus $(C, F, I, M, P, S)$. In D-F and N-P, respectively, the borders of the $C N$, putamen $(P), N A(D, N), A B N A, B N A$ and $L N A(E, O)$, and MDNT $(F, P)$ are indicated. The high-power photomicrographs showing neurons in the $N A(G, K), L N A(H, L)$ and MDNT $(l, M)$ are representative of the magnification at which the stereological estimates were obtained. Scale bar $=23 \mathrm{~mm}$ in A -C and Q $-5,10 \mathrm{~mm}$ in D-F and N-P, and $50 \mu \mathrm{m}$ in G-M. 


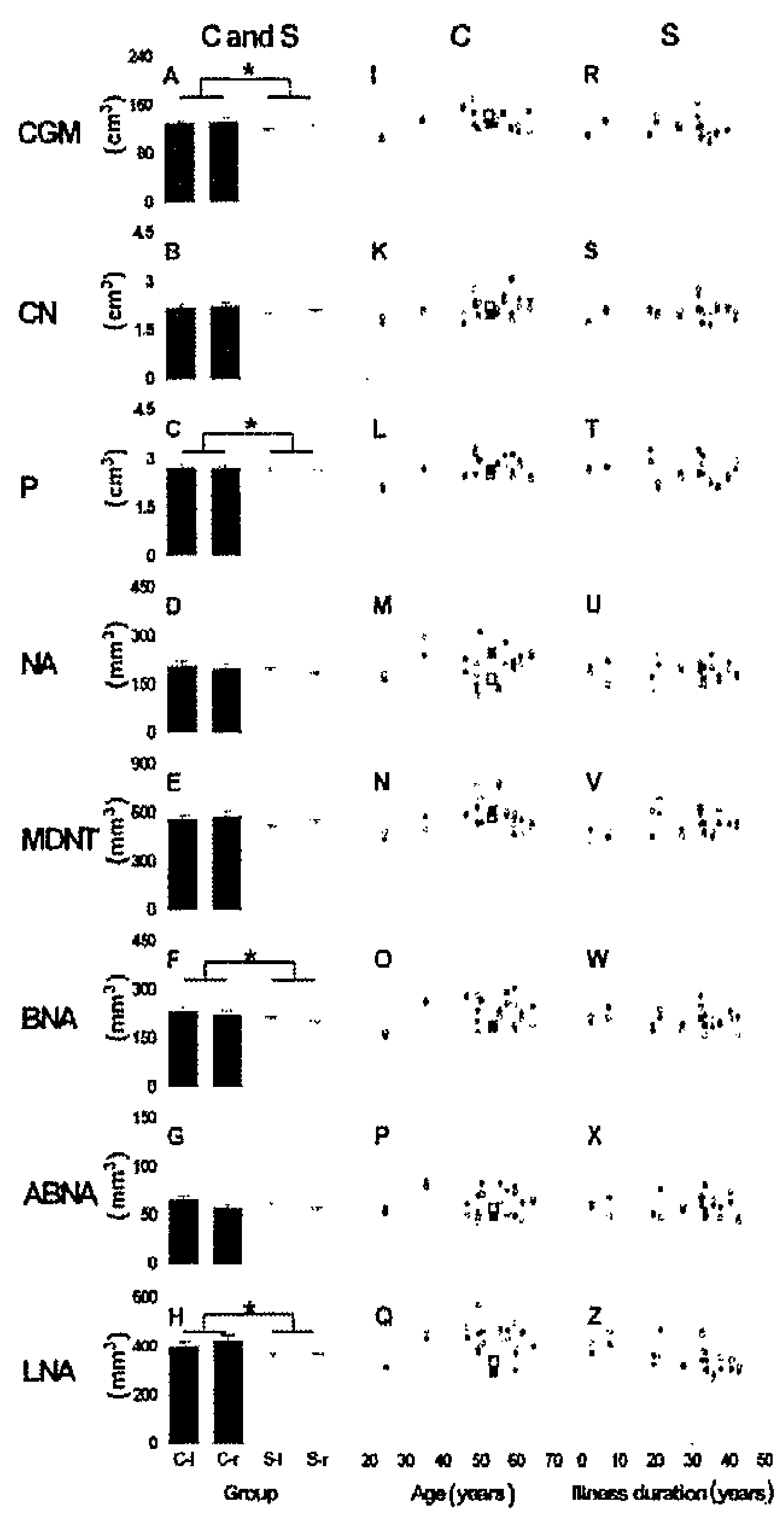

Fig. 2. Volumes of $C G M(A, I, R), C N(B, K, S)$, putamen $(P ; C, L, T), N A(D, M, U), M D N T(E, N, V)$, BNA $(F, O, W), A B N A(G, P, X)$ and $L N A(H, Q, Z)$ in both hemispheres of the brains from 13 male schizophrenic patients ( $S$; open bars in $A-H$, and dots in $R-Z$ ) and 13 age-matched controls $\left[C_{\text {; }}\right.$ closed bars in $\mathrm{A}-\mathrm{H}$, and dots in $\mathrm{I}-\mathrm{Q}$; squares in case of the results obtained for the single brain embedded in celloidin (C7)]. In A-H, data are shown as mean and standard error of the mean for the left (I) and right $(r)$ hemispheres from schizophrenic patients (S-I and $S-r$ ) and controls $(C-I$ and $C-r$ ). In I $-Z$, individual data for the left hemispheres (closed dots or squares, respectively) and right hemispheres (open dots or squares, respectively) from controls $(I-Q)$ and schizophrenic patients (R-Z) are shown as function of the control patients' age (or the illness duration of the schizophrenic patients, respectively). ${ }^{*} \mathrm{P}<0.05$ for the fixed factor Diagnosis in general linear model MANOVA. 
Chapter 2

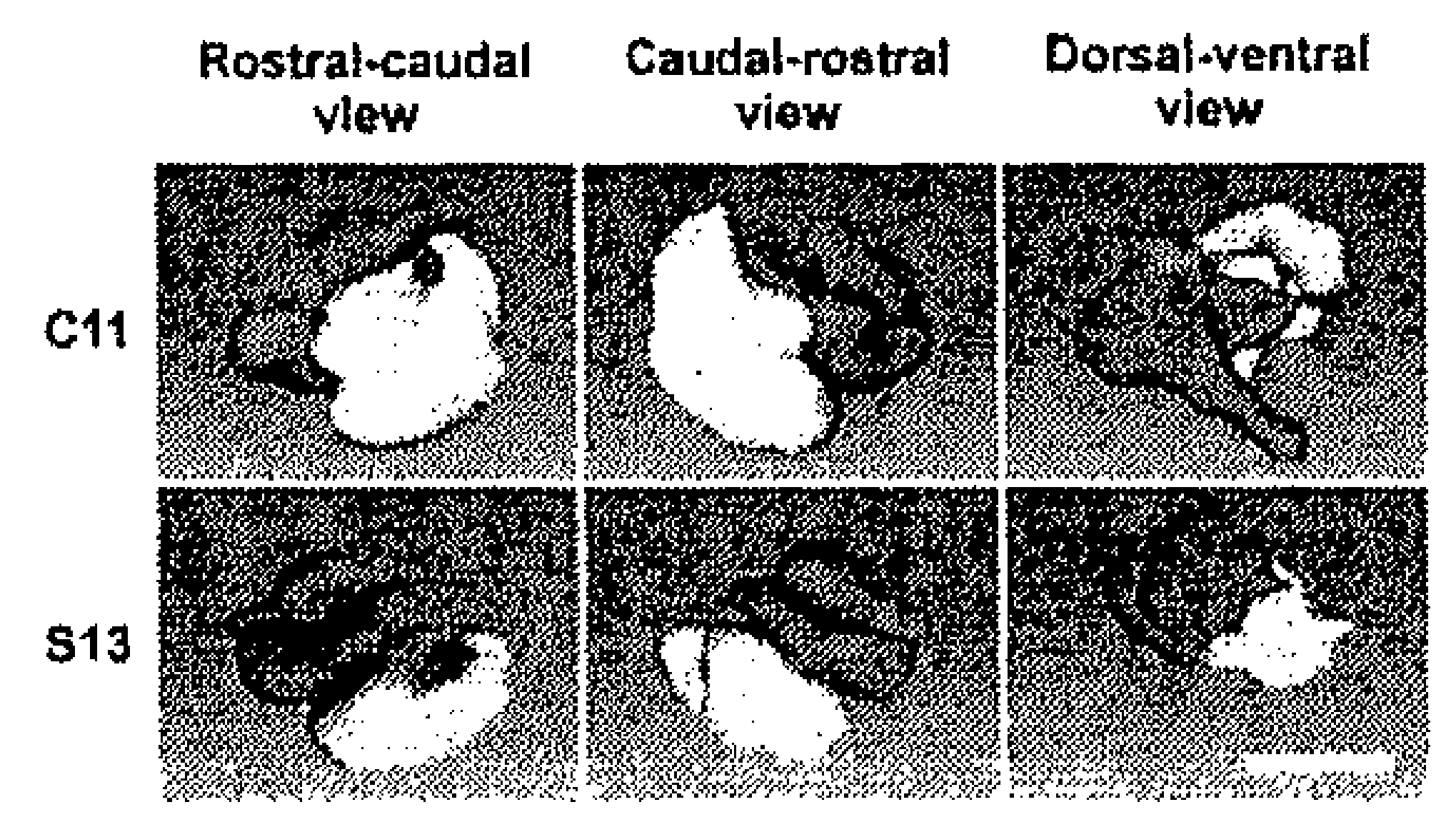

Fig. 3. Reconstructions of the LNA (green), BNA (red) and ABNA (magenta) in the right hemisphere from the control $C 11$ ( 60 years old) and from the schizophrenic patient $S 13$ (64 years old) in rostral -caudal view (left column), caudal - rostral view (middle column) and dorsal - ventral view (right column). Compared with the volume of the LNA from C11 (set to 100\%), the relative volume of this nucleus was $71.2 \%$ in 513 . For the BNA, the corresponding values were $76.9 \%(S 13 / C 4)$ and for the ABNA, $62.8 \%$. Scale bar $=1 \mathrm{~cm}$.

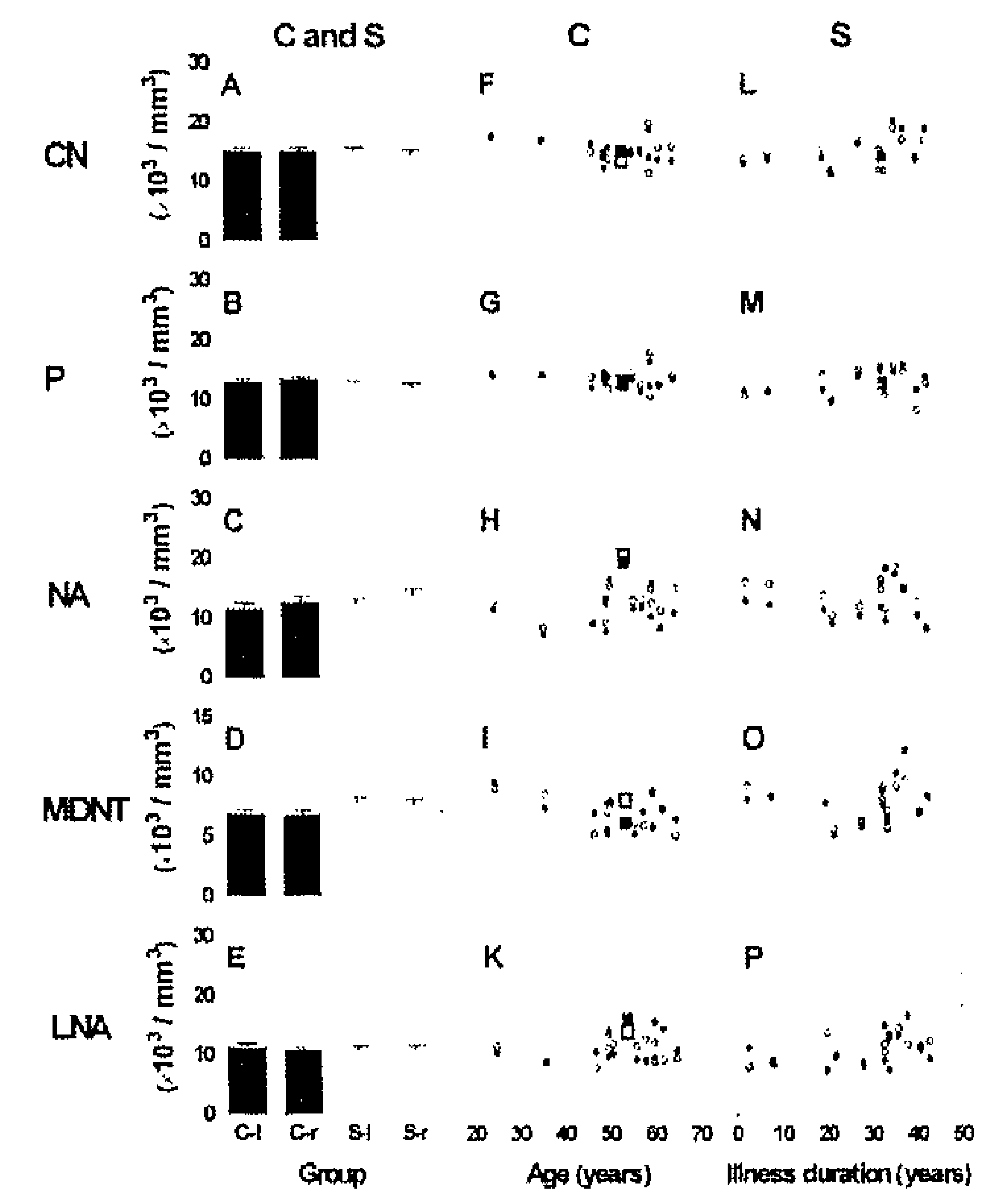

Fig. 4. Neuron density in $C N(A, F, L)$, putamen $(P ; B, G, M), N A(C, H, N), M D N T(D, l, O)$ and $L N A(E$, $K, P$ ) in both hemispheres of the brains from 13 male schizophrenic patients ( $S$; open bars in $A \wedge E$, and dots in L-P) and 13 age-matched controls $\left[C_{i}\right.$ closed bars in $A-E$, and dots in $F-K$; squares in case of the results obtained for the single brain embedded in celloidin (C7)]. In A - E, data are shown as mean and standard error of the mean for the left $(I)$ and right $(r)$ hemispheres from schizophrenic patients (S-I and S-r) and controls ( $C-1$ and $C-r$ ). In F-P, individual data for the left hemispheres (closed dots or squares, respectively) and right hemispheres (open dots or squares, respectively) from controls $(F-K)$ and schizophrenic patients $(L-P)$ are shown as function of the control patients'age (or the illness duration of the schizophrenic patients, respectively). 


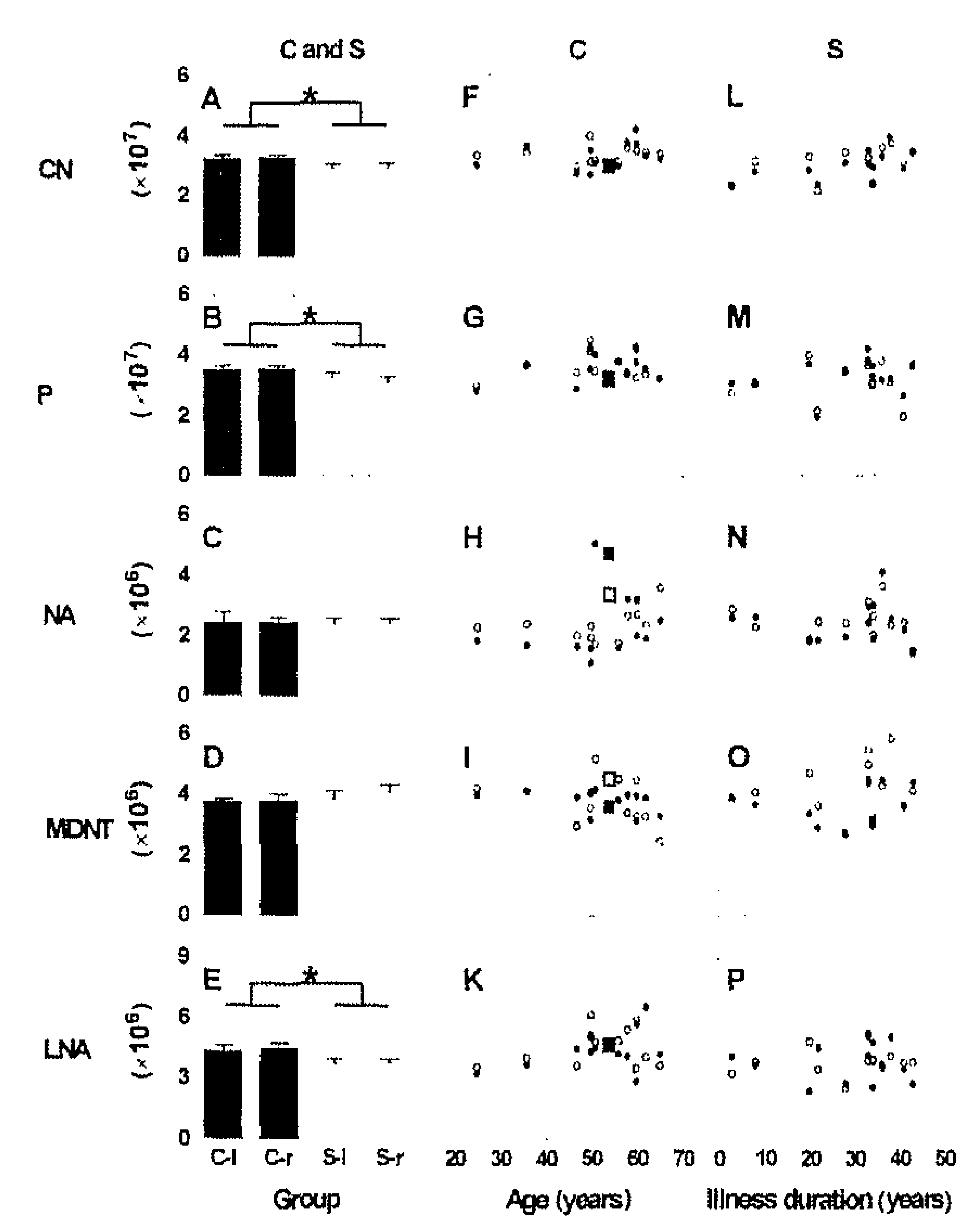

Fig. 5. Total neuron number in $C N(A, F, L)$, putamen $(P ; B, G, M), N A(C, H, N), M D N T(D, I, O)$ and LNA $(E, K, P)$ in both hemispheres of the brains from 13 male schizophrenic patients ( $S$; open bars in $A-E$, and dots in L-P) and 13 age-matched controls [C; closed bars in A-E, and dots in F-K; squares in case of the results obtained for the single brain embedded in celloidin (C7)]. In A-E, data are shown as mean and standard error of the mean for the left $(I)$ and right $(r)$ hemispheres from schizophrenic patients (S-I and S-r) and controls $(C-I$ and $C-r$ ). In F -P, individual data for the left hemispheres (closed dots or squares, respectively) and right hemispheres (open dots or squares, respectively) from controls $(F-K)$ and schizophrenic patients $(L-P)$ are shown as function of the control patients' age (or the illness duration of the schizophrenic patients, respectively). ${ }^{*} P<0.05$ for the fixed factor diagnosis in general linear model MANOVA. 


\section{Crarea 3}

\section{Microvessel length density, total length, and length per neuron in five subcortical regions in schizophrenia}

Pawel Kreczmanski ${ }^{1,2}$, Helmut Heinsen ${ }^{3}$, Valentina Mantua ${ }^{1,4,5}$ Fritz Woltersdorf6, Thorsten Masson ${ }^{7}$, Norbert Ulfig ${ }^{6}$, Rainald Schmidt-Kastner ${ }^{1}$ Hubert Korr ${ }^{1,2}$, Harry W.M. Steinbusch ${ }^{1,2}$, Patrick R. Hof ${ }^{8}$, and Christoph Schmitz ${ }^{1,2}$

'School for Mental Health and Neurosciences, Division of Cellular Neuroscience, Maastricht University, Maastricht, The Netherlands 2European Graduate School of Neuroscience (EURON), Maastricht, The Netherlands

${ }^{3}$ Morphological Brain Research Unit Psychiatric Clinic, University of Wuerzburg, Wuerzburg, Germany

${ }^{4}$ Department of Psychiatry, Section of Clinical Neuropharmacology, Institute of Psychiatry, Kings College London, London, United Kingdom

'Department of Psychiatric Sciences and Psychological Medicine,

Psychiatric Clinic Ill, University of Rome La Sapienza, Rome, Italy

${ }^{6}$ Department of Anatomy, University of Rostock, Rostock, Germany

'Department of Anatomy and Cell Biology, RWTH Aachen University, Aachen, Germany

${ }^{8}$ Department of Neuroscience, Mount Sinai School of Medicine,

New York, NY, USA

Acta Neuropathol. 2009 Apr;117(4):409-21. Epub 2009 Feb 6 


\begin{abstract}
Previous studies (Prabakaran et al. [2004] Mol Psychiat 9:684-697; Hanson and Gottesman [2005] BMC Med Genet 6:7) have suggested that microvascular abnormalities occur in the brain of patients with schizophrenia. To assess the integrity of the microvasculature in subcortical brain regions in schizophrenia, we investigated the microvessel length density, total microvessel length, and microvessel length per neuron using design-based stereologic methods in the caudate nucleus, putamen, nucleus accumbens, mediodorsal nucleus of the thalamus, and lateral nucleus of the amygdala in both hemispheres of 13 postmortem brains from male patients with schizophrenia and 13 age-matched male controls. A general linear model multivariate analysis of variance with diagnosis and hemisphere as fixed factors and illness duration (patients with schizophrenia) or age (controls), postmortem interval and fixation time as covariates showed a number of statistically significant differences in the brains from the patients with schizophrenia compared to the controls. There was an increased mean microvessel length density in the caudate nucleus $(+6.3 \%$ on the left side $[\mathrm{l}]$ and $+5.8 \%$ on the right side $[\mathrm{r}])$ and the mediodorsal nucleus of the thalamus $(1:+4.3 \%, r:+5.1 \%)$, and a reduced total microvessel length in the lateral nucleus of the amygdala (1: $-17.5 \%, \mathrm{r}:-10.8 \%)$. No significant differences between patients with schizophrenia and controls were found for the microvessel length per neuron in any of the investigated regions. These data extend our earlier findings in prefrontal cortex area 9 and anterior cingulate cortex area 24 from the same brains (Kreczmanski et al. [2005] Acta Neuropathol 109:510-518), that alterations in microvessel length density, total length, and particularly length per neuron cannot be considered characteristic features of schizophrenia. As such, compromised brain metabolism and occurrence of oxidative stress in the brains of patients with schizophrenia are likely caused by other mechanisms.
\end{abstract}




\section{Introduction}

Schizophrenia is a mental illness characterized by psychosis, cognitive impairment and social withdrawal, and is the most disabling among psychiatric disorders [58]. A combination of genetic susceptibility and environmental perturbations appears to be necessary for the expression of the disease $(1,30,66,69,73)$. However, the mechanisms involved in the pathogenesis of schizophrenia remain elusive $[29,30,69,73]$. Possibilities include alterations of highly specific developmental processes at the synaptic level $[28,52]$, abnormalities in myelination $[26,71]$, as well as global metabolic changes of brain function and oxidative stress $[2,8,56]$. Subtle metabolic deficits such as hypoxia have long been suspected to cause widespread neuronal dysfunction in schizophrenia [42, 43] (see also [44]). Because the function of neurons depends on adequate oxygen and energy substrate supply, subtle changes in vascular integrity may play a role in the development of schizophrenia. Routine neuropathological observations indicate that there are no differences in the frequency of large or small blood vessel alterations in the brain of patients with chronic schizophrenia compared to controls [3] but there are no systematic studies of the brain microvasculature system in this disease. Whereas a genetic and inflammatory vascular mechanism has been proposed for schizophrenia [27], neuropathological evidence for inflammatory vascular changes in the brain of patients with schizophrenia is lacking. Furthermore, studies of regional cerebral blood flow ( $\mathrm{rCBF}$ ) in the frontal cortex of patients with schizophrenia under resting conditions have yielded inconsistent results [16, 54, 64]. Following to the pioneering studies of Ingvar and Franzen [39] and Franzen and Ingvar [24] a number of abnormalities in the coupling between task-related increases in brain activity and rCBF have been described in frontal cortical regions of patients with schizophrenia $[2,33,55$, 76] but these changes were generally attributed to primary neuronal dysfunction. Imaging studies with dynamic susceptibility contrast MRI pointed to an increase of cerebral vascular volume in patients with schizophrenia and postulated abnormalities of the configuration of cerebral vessels [17] whereas a more recent study found a decrease of cerebral vascular volume in schizophrenia [11].

A recent study employing a combined transcriptomics, proteomics, and metabolomics approach found evidence for mitochondrial dysfunction, compromised brain metabolism, and oxidative stress in the frontal cortex of patients with schizophrenia [61], thereby confirming and expanding earlier reports [8,56]. Prabakaran and colleagues [61] hypothesized that the observed alterations can be explained by abnormalities of the microvasculature in schizophrenia.

In a previous study, we analyzed the microvessel length density in prefrontal cortex area 9 and anterior cingulate cortex area 24 (both areas are involved in the neuropathology of schizophrenia, as reviewed in [28]), with a design-based stereology approach, and found no differences between patients with schizophrenia and controls [46]. In the present study we extended these investigations by analyzing the microvessel length density in the caudate nucleus, putamen, nucleus accumbens, mediodorsal nucleus of the thalamus, and lateral 
nucleus of the amygdala, structures that are also considered to be involved in the neuropathology of schizophrenia $[7,9,13,18,19,22,47,50,59,60,77]$. To this end, we used the same sample of brains from patients with schizophrenia and controls that was used in our earlier study [46]. In a parallel study, we have already investigated these five subcortical regions for their volume, total number of neurons, and neuron density [47]. Based on these data we could also assess possible changes in total microvessel length and length per neuron in these five subcortical regions.

\section{Materials and Methods}

\section{Brain specimens}

This study was performed on postmortem brains (both hemispheres) from 13 male patients with schizophrenia (mean age $51.5 \pm 3.3$ years; mean postmortem interval [time between death and autopsy] $27.5 \pm 6.0$ hours; mean fixation time $912 \pm 372$ days; data given as mean $\pm S E M$ ) and from 13 age-matched male controls (mean age $51.9 \pm 3.1$ years; mean postmortem interval $23.7 \pm 3.8$ hours; mean fixation time $247 \pm 53$ days). The same brains were investigated in our previous studies for mean cell spacing abnormalities in the neocortex [15], microvessel length densities in the frontal cortex [46], and volume, neuron density, and total neuron number in the caudate nucleus, putamen, nucleus accumbens, mediodorsal nucleus of the thalamus, and lateral nucleus of the amygdala [47]. The age of the patients, illness duration, clinical diagnoses, causes of death, the postmortem interval and the fixation time are summarized in Table 1 . The patients with schizophrenia did not differ from the controls with respect to mean age (Student's two-tailed $t$ test; $p=0.946$ ), mean postmortem interval $(\mathrm{p}=0.581)$, and mean fixation time $(\mathrm{p}=0.089)$. All patients with schizophrenia had been patients either in German university hospitals or in German State psychiatric hospitals (6 hospitals including one university hospital in which some control patients were also treated for nonpsychiatric or neurologic illnesses) and full clinical records were available. All controls had been patients either in German university hospitals or in German local district hospitals ( 5 hospitals including one university hospital in which patients with schizophrenia were also treated). Records from autopsy (including a summary of the medical history) were available for all patients with schizophrenia and all controls. All pathologists involved in the autopsies were instructed by H.H. and adhered to identical handling and processing conditions of the brains. All patients with schizophrenia met the Diagnostic Statistical Manual, 4th revision (DSM-IV) and International Statistical Classification of Diseases and Related Health Problems, 10th revision (ICD-10) diagnostic criteria. The clinical notes were assessed by two experienced clinical psychiatrists to ensure that the brains from the controls were free from psychopathology, and for clear evidence that the diagnosis of patients with schizophrenia was concordant with DSM-IV criteria for schizophrenia. The mean age at onset was $22.9 \pm 1.5$ years. Exclusion criteria for both patients with schizophrenia and controls comprised neurological problems that required 
intervention or interfered with cognitive assessment (e.g., stroke with aphasia), history of recurrent seizure disorder, history of severe head injury with loss of consciousness, diabetes mellitus with free plasma glucose $>200 \mathrm{mg} / \mathrm{dl}$, and history of drug or alcohol abuse. Patients with schizophrenia and controls were similar in terms of the ethnic backgrounds. However, they were not fully matched for socio-economical status and education, which would have placed severe constraints on our sample. Moreover, all patients with schizophrenia were subjected to long-term treatment with typical neuroleptics (because most of the patients were not hospitalized throughout the duration of their illness and their clinical records did not cover fully the entire medication histories, it was not possible to calculate lifetime medication exposures). In all of the cases, autopsy was performed after consent was obtained from a relative according to the laws of the Federal Republic of Germany. The use of these autopsy cases for scientific investigations as outlined here has been approved by the relevant Institutional Review Boards. Brains were fixed by immersion in $10 \%$ formalin (1 part commercial $40 \%$ aqueous formaldehyde in 9 parts $\mathrm{H}_{2} \mathrm{O}$ ) prior to histologic processing. Fixation and tissue processing was performed at the Morphological Brain Research Unit, University of Wuerzburg (Germany) under identical conditions for all brains (except for brain $\mathrm{C} 7$ that was embedded in celloidin instead of gelatin).

\section{Tissue processing and immunohistochemistry}

After separating the brainstem with the cerebellum from the forebrain and dividing the hemispheres mediosagittally, both hemispheres were cut into entire series of 440-to 700$\mu \mathrm{m}$ thick coronal sections (for details see [47]). Every second or third section was stained with gallocyanin (a Nissl stain) as previously described [31]. From the remaining sections, two each showing the striatum, the thalamus and/or the amygdala were randomly selected, and approximately $5 \times 5-\mathrm{cm}$ small pieces containing these regions were cut out. After rinsing in tap water for several hours, these pieces were stored in Tris-buffered saline (TBS) overnight $(\mathrm{pH}=9.0)$ at room temperature. Then they were placed in TBS $(\mathrm{pH}=9.0)$ and heated in a microwave oven for $15 \mathrm{~min}$ at $90^{\circ} \mathrm{C}$ for antigen retrieval. After cooling down tissue pieces were rinsed in TBS twice $(\mathrm{pH}=7.4)$ and were placed in TBS based $30 \%$ sucrose for cryoprotection. Pieces were then frozen and cut into $50 \mu$ m-thick coronal sections on a cryostat (Type HM 500 OMV, Microm, Walldorf, Germany). One $50 \mu \mathrm{m}$-thick section per piece was mounted on a glass slide, dried, and stained with cresyl violet $(0.01 \%, 25 \mathrm{~min})$. Slides were dehydrated and coverslipped using DPX (Serva, Heidelberg, Germany). These $50 \mu \mathrm{m}$-thick sections stained with cresyl violet as well as the adjacent 440-700 $\mu \mathrm{m}$-thick sections stained with gallocyanin were used to identify the caudate nucleus, putamen, nucleus accumbens, mediodorsal nucleus of the thalamus, and lateral nucleus of the amygdala according to established criteria in the literature (caudate nucleus and nucleus accumbens: $[12,37,49,51]$; mediodorsal nucleus of the thalamus: $[21,22,32,34,41,60]$; lateral nucleus of the amygdala: $[10,65,67,68]$. In the case of the mediodorsal nucleus of the thalamus, the magnocellular, parvocellular and densocellular regions as well as the caudodorsal subdivision were included in the delineations, as 
also done in other studies $[13,22,60]$. The boundaries of the investigated brain regions were identified on all sections showing the corresponding region using an Olympus SZX9 stereo microscope (Olympus; Tokyo, Japan) and were marked on the back side of the glass slides with a felt-tip pen. Identification and delineation of boundaries was performed by H.H. (caudate nucleus, putamen), V.M. (nucleus accumbens), T.M. (mediodorsal nucleus of the thalamus) and F.W. (lateral, basal and accessory basal nuclei of the amygdala). Crossvalidation (and, if necessary, slight modifications of the delineations) was performed by C.S. (caudate nucleus, putamen) and C.S., H.H. and P.R.H. (all other brain regions). The remaining $50 \mu \mathrm{m}$-thick sections were stored in TBS ( $\mathrm{pH}=7.4$ ) at $4^{\circ} \mathrm{C}$.

For immunohistochemical processing, another $50 \mu \mathrm{m}$-thick section per piece (freefloating sections) was rinsed in TBS after each step of the incubation procedure. Nonspecific background staining was blocked by two preincubation steps: first, $10 \%$ methanol and $7 \%$ hydrogen peroxide in TBS (30 min), and then $1.5 \%$ lysine, $0.25 \%$ Triton X-100 and $10 \%$ bovine serum albumin (BSA) in TBS (60 min) (all from Sigma-Aldrich; St. Louis, MO, USA). Then, sections were incubated with mouse monoclonal anti-collagen IV primary antibody (\#C1926, clone COL-94; Sigma) at $4^{\circ} \mathrm{C}$ for 48 hours. Antibody dilution was 1:500 in TBS containing 2\% BSA. Afterwards sections were incubated with secondary antibody (biotinylated anti-mouse IgG 1:200, Jackson ImmunoResearch, West Grove, PA, USA; secondary antibody diluted in TBS containing $0.2 \%$ donkey serum, Sigma) for 2 hours at room temperature, and then with avidinbiotin peroxidase complex ( $A B C$ Vectastain; 1:25 in TBS; Sigma) for another 2 hours. 3,3'diaminobenzidine (DAB; $0.8 \mathrm{mg} /$ $\mathrm{ml}$ with $0.003 \%$ hydrogen peroxide; Sigma) was used as a chromogen. To prevent shrinkage in section thickness, sections were not dehydrated but mounted on gelatin-coated slides and coverslipped with $80 \%$ glycerol in TBS. Note that the actual average section thickness after immunohistochemical processing was $45.7 \mu \mathrm{m}$ (see also Table 2) as determined during the stereologic analyses described in detail below. Accordingly, shrinkage in section thickness was less than $10 \%$. This was important because the space balls method used to estimate microvessel length density is sensitive to shrinkage in section thickness [25].

\section{Stereologic analyses}

Stereologic analyses were performed with a stereology workstation, consisting of a modified light microscope (Olympus BX50 with PlanApo objective $1.25 \times[$ N.A. $=0.04]$ and UPlanApo objectives $10 \times$ [N.A. $=0.4$ ], 20x [oil; N.A. $=0.8$ ] and 40x [oil; N.A = 1.0]; Olympus, Tokyo, Japan), motorized specimen stage for automatic sampling (Ludl Electronics; Hawthorne, NY, USA), electronic microcator (Heidenhain, Traunreut, Germany), CCD colour video camera (HVC20AMP; Hitachi, Tokyo, Japan), and stereology software (StereoInvestigator; MicroBrightField, Williston, VT, USA).

Microvessel length density was investigated with the Space Balls method [14, 57] (see also Fig. 8 in [63]) (Fig. 1). Briefly, the $50 \mu \mathrm{m}$-thick sections stained with cresyl violet were laid onto the corresponding adjacent sections processed with immunohistochemistry and were exactly aligned under the microscope (the $50 \mu \mathrm{m}$-thick sections stained with cresyl 
violet did not show differences in shrinkage in direction $\mathrm{X}$ and $\mathrm{Y}$ compared to the sections processed with immunohistochemistry). Then the sections stained with cresyl violet were brought into focus, and the five regions of interest were delineated by tracing their boundarics on each section on video images displayed on the monitor of the stereology workstation. Afterwards the sections stained with cresyl violet were removed, and the sections processed with immunohistochemistry were brought into focus. Then hemispheres with a radius of $30 \mu \mathrm{m}$ (generated by the stereology software while focussing through the section thickness) were systematically and randomly placed within the sections through the regions of interest (with a guard zone of $5 \mu \mathrm{m}$ between the upper surface of the section and the top of the hemispheres; details are provided in Table 2). The microvessel length density was obtained from the total number of intersections between the hemispheres and the microvessels (data given in Table 2) using the following formula derived from the description of the Space Balls method in the literature $[14,57]$ according to the sampling scheme applied in the present study and considering the area of the delineated regions of interest and the actual section thickness after tissue processing (see also [46]):

$$
C L D=\frac{2 \times \sum i s\left(D_{X} \times D_{Y} \times t\right)}{2 \times \pi \times r^{3}} \times \frac{1}{V}
$$

where CLD represents the microvessel length density, $\sum$ is the total number of intersections between the hemispheres and the microvessels, DX and DY the distance between the midpoints of the centers of the hemispheres in the $X$ and $Y$ axes, $t$ the actual average section thickness after histologic processing, $r$ the radius of the hemispheres and $V$ the investigated tissue volume. It is important to note that these microvessel length density estimates were based on the analysis of thick sections in three dimensions (3D) and therefore not dependent on the plane of section, the spatial arrangement of the microvessels or their diameter. To focus the analysis on microvessels and to exclude arterioles and venules, the diameter of all vessels coming into view during the space ball analysis was measured with the "Fast measure line" tool of the stereology software, and intersections between the (space ball) hemispheres and vessels with a diameter greater than $9 \mu \mathrm{m}$ were not counted. In addition, we always focused on the middle of a given vessel's diameter when recording intersections between the Space Ball hemispheres and the microvessel in order to minimize the Space Ball bias due to the non-zero diameter of the vessels [25]. The actual section thickness after immunohistochemical processing was measured at the position of every 10th Space Ball, yielding on average approximately 40 measures per section. Note that the actual section thickness after immunohistochemical processing (on average $45.7 \mu \mathrm{m}$ ) guaranteed that the Space Balls were placed always fully within the section thickness. Finally, total microvessel lengths and lengths per neuron were calculated individually for each subcortical region 
as the product of the microvessel length density in and the volume of the region (total microvessel lengths), and as the ratio of the total microvessel length and the total neuron number in the region (microvessel lengths per neuron), respectively. Volume and total neuron number data were taken from [47]. Furthermore, it was tested whether there was a principal correlation between the neuron density and microvessel length density when all subcortical structures were combined.

\section{Statistical analysis}

For both patients with schizophrenia and controls, mean and standard error of the mean were calculated for all investigated variables separately for the left and the right hemispheres. Comparisons between patients with schizophrenia and controls were performed using generalized linear model multivariate analysis (MANOVA), with diagnosis and hemisphere as fixed factors and the following variables as covariates: (i) the adjusted illness duration of the patients with schizophrenia (calculated as individual age at death minus age at onset plus the mean age at onset of all patients with schizophrenia) or the age of the controls, respectively, (ii) the postmortem interval and (iii) the fixation time (note that use of the actual individual illness duration of the patients with schizophrenia instead of the adjusted ones as covariate would have caused invalid results of the MANOVA model because there was no illness duration of the controls, and the mean illness duration of the patients with schizophrenia was significantly different from the mean age of the controls [Student's twotailed $t$ test; $p<0.001]$ whereas the mean adjusted illness duration was not $[p=0.974])$. For each investigated variable all investigated brain regions were tested simultaneously. Post-hoc tests in the analyses of covariance were performed with linear regression analysis. In addition, the microvessel length per neuron data from both hemispheres of patients with schizophrenia and controls were pooled for each subcortical region, and a comparison among the five investigated regions was performed with one-way analysis of variance (ANOVA) followed by post-hoc Bonferroni tests for pairwise comparisons. In all analyses an effect was considered statistically significant if its associated $\mathrm{p}$ value was smaller than 0.05 . Linear regression analysis was carried out between neuronal densities and microvessel length densities across the five subcortical structures. Calculations were performed using SPSS (Version 12.0.1 for Windows, SPSS, Chicago, IL).

\section{Photography}

Photomicrographs shown in Fig. 1 and 2 were produced using an Olympus DP 70 digital camera attached to an Olympus AX 70 microscope and cellP software Version 2.3 (Soft Imaging System, Münster, Germany). The final figures were constructed using Corel Photo-Paint v.11 and Corel Draw v.11 (Corel, Ottawa, Canada). Adjustments of contrast and brightness were made to facilitate recognition of the immunohistochemical signal at low and high magnification, without altering the appearance of the original materials. 


\section{Results}

No major abnormalities such as irregular size of the vascular wall, stenosis, or dilation were noted in the immunohistochemical preparations for collagen IV in the materials from the patients with schizophrenia (Fig. 2). Compared to the controls, the patients with schizophrenia showed a statistically significant increase in mean microvessel length density in the caudate nucleus $(+6.3 \%$ on the left side $[1]$ and $+5.8 \%$ on the right side $[\mathrm{r}] ; \mathrm{F}(1)$ [one degree of freedom $]=4.869$ and $\mathrm{p}=0.034$ for the fixed factor diagnosis in the MANO$\mathrm{VA})$, and in the mediodorsal nucleus of the thalamus $(1:+4.3 \%, \mathrm{r}:+5.1 \% ; \mathrm{F}(1)=4.857$, $\mathrm{p}=0.034$ ) (Fig. 3; Table 3 shows all $\mathrm{P}$ values of the MANOVAs performed).

The patients with schizophrenia had a significantly reduced mean total microvessel length in the lateral nucleus of the amygdala (l: $-17.5 \%, r:-10.8 \% ; F(1)=6.553$, $\mathrm{p}=0.015$; Fig. 4). In contrast, there were no significant differences between the patients with schizophrenia and the controls with respect to the mean microvessel length per neuron in the investigated subcortical regions (Fig. 5). Also, no significant differences between the left and the right hemispheres were found for the investigated variables in both patients with schizophrenia and controls ( $p>0.05$ for the fixed factor hemisphere in all MANOVAs performed). The mean microvessel length per neuron differed among the five investigated subcortical regions, in the order mediodorsal nucleus of the thalamus $>$ lateral nucleus of the amygdala $>$ putamen $>$ caudate nucleus $>$ nucleus accumbens $(p<0.001)$. Except for the comparison between the caudate nucleus and the nucleus accumbens, all pairwise comparisons showed significant differences between the regions $(p<0.01$ for the comparison between caudate nucleus and putamen, and $\mathrm{p}<0.001$ for all other comparisons).

Postmortem changes are known to affect stereologic estimates of cerebral blood vessels [38]. In the present study, the postmortem interval had a significant effect on the total microvessel length in the putamen $(F(1)=4.864, P=0.034$; Fig. 6). A significant effect of the brain's fixation time on the total microvessel length in the mediodorsal nucleus of the thalamus was also found $(\mathrm{F}(1)=4.812, \mathrm{p}=0.035)$. However, post-hoc linear regression analysis revealed no significant correlations between the postmortem interval and the fixation time and any of the investigated variables. These results suggest that the alterations in mean microvessel length densities and mean total microvessel lengths found in the investigated subcortical regions in the patients with schizophrenia were not caused by the control patients' age (or the illness duration of the patients with schizophrenia), the postmortem interval or the fixation time of the brains. In addition, no correlation was found between the microvessel length density and the neuron density in the five investigated subcortical regions, either in the patients with schizophrenia, or in the controls (controls: $\mathrm{r} 2=0.006 ; \mathrm{p}=0.900$; patiens with schizophrenia: $\mathrm{r} 2=0.054$; $\mathrm{p}=0.708$; Fig. 7). Finally it should be mentioned that the results obtained for the single brain embedded in celloidin (C7) showed no systematic deviation from the results obtained for the other brains (Figs. 3, 4 and 5). 


\section{Discussion}

The present data reveal a small increase in microvessel length density in the caudate nucleus and in the mediodorsal nucleus of the thalamus, and a decrease in mean total microvessel length in the lateral nucleus of the amygdala in schizophrenia. Also, as the total neuron numbers were assessed in the same subcortical regions in this brain series [47], microvessel length per neuron could be calculated. There were no changes in the mean microvessel length per neuron in any of the five subcortical structures in the brains from the patients with schizophrenia, suggesting that modest changes in neuron number are likely to be paralleled by minor vascular changes. It should be noted that the results reported in the present study do not reflect absolute values found in the brain of living humans because postmortem brains show shrinkage during formalin fixation. Accordingly, the results presented here should only be used to interpret potential differences between patients with schizophrenia and matched controls whose postmortem brains were processed under exactly identical conditions. In this regard one could speculate that postmortem brains from patients with schizophrenia may shrink differently than postmortem brains from controls, but there is no available evidence for this in the literature.

The present study confirms and extends observations of normal microvessel lengths in the prefrontal cortex area 9 and anterior cingulate cortex area 24 in schizophrenia [46]. These data do not support the hypothesis of alterations in the microvasculature in chronic schizophrenia. Because these studies were performed in regions considered to represent "hot spots" for structural pathology in schizophrenia, the present negative outcome indicates that there might be no anatomical substrate at the microvascular level for abnormal rCBF and cerebral blood volume, alterations reported in the brains of patients afflicted by schizophrenia $[2,11,17,24,33,55,76]$.

A survey of gene expression studies with microarrays in the brain of patients with schizophrenia showed changes in a few genes primarily associated with the vasculature, although no consistent directional trends emerged [62]. A hypothesis paper suggested that genetic factors and inflammatory responses of cerebral vessels could underlie some of the changes seen in schizophrenia [27]. However, the data of the present study altogether do not support the hypothesis of involvement of brain microvessels that would be otherwise expected if a major inflammatory process took place in schizophrenia. Because only vessels with a maximal diameter of $9 \mu \mathrm{m}$ were counted in the present study, subtle abnormalities may still occur upstream in arterioles, involving vascular smooth muscle cells and regulation of tissue perfusion. Indeed, changes in the activity-dependent regulation of $\mathrm{rCBF}$ have been observed in the prefrontal cortex in schizophrenia [24, 76]. However, hemodynamic responses appear to be normal in visual, motor and somatosensory cortices in this disorder [5]. It is unlikely that primary differences in the structure and function of arterioles occur selectively in some cortical regions, and therefore changes in $\mathrm{rCBF}$ activation most likely are due to region-specific neuronal or glial factors. In addition, it appears that changes in gene and protein expression found in cortical samples from chronic schizo- 
phrenia patients probably reflect an abnormal metabolic state [61]. This could be equally due to the primary disease process and to secondary long-term neuronal dysfunction, chronic medication, or imbalances in agonal events influencing microarray measurements $[40,53,72,74]$. It remains to be shown whether the microvasculature of the white matter in schizophrenia is normal, as several recent findings point to subtle white matter changes in schizophrenia $[20,35,36]$, and in view of the vulnerability of the white matter to prolonged hypoperfusion [23]. Furthermore, an abnormal decrease in perivascular labeling of GFAP in astrocytes has been reported in the white matter in schizophrenia [75].

As a side observation it is of interest that the neuron density was not correlated with the microvessels length density when five subcortical regions were combined for analysis in controls or in patients with schizophrenia. Baborie and Kuschinksy [4] did not find a correlation between the density of all nuclei and microvessel densities across multiple areas of the rat brain. It is likely that the microvessel length density is linked to metabolic expenditures in neuronal and glial processes whereby neuron density (measured in the present study) or overall cell density (measured in [4]) do not tightly correlate with the total amount of functional processes in each structure. As all patients were treated with neuroleptics, we cannot exclude that the lack of changes in microvessel measurements was due to the treatment; in other words, untreated patients with schizophrenia could have an abnormal microvasculature. However, the influence of neuroleptics on brain microvessels in chronically treated patients with schizophrenia is presently unknown. Dopaminergic processes make close contacts with capillaries in the prefrontal cortex and dopamine reduces local perfusion [48]. Dopamine has been shown to have anti-angiogenic properties in peripheral tissues, tumors, and placenta $[6,45,70]$. Thus, one cannot exclude that abnormal dopamine levels in schizophrenia may have functional and structural effects on cerebral microvasculature before the onset of clinical symptoms that may be masked by chronic dopamine receptor blockade with neuroleptics. Further research will be necessary in this regard.

Finally it should be mentioned that the present observations in chronic schizophrenia cases must be separated from the concept that abnormal interactions between hypoxia, the microvasculature and developing neurons could be important for the neurodevelopmental perturbations that are thought to underlie schizophrenia [62]. Specifically, one cannot exclude the possible occurrence of alterations in microvessel length and microvessel length per neuron in the fetal brain of people who will develop schizophrenia later in life. If this were the case, however, these alterations do not persist during postnatal life in the brains from patients with schizophrenia. Rather, compromised brain metabolism and occurrence of oxidative stress in the brains from patients with schizophrenia are likely caused by other mechanisms. 


\section{Acknowledgements}

We thank E.K. Broschk and He. Steinbusch for expert technical assistance, Dr. M. Schüler (Bayreuth, Germany) for valuable help in retrieving clinical data of the investigated patients, and

G. Greene and B. Boehringer (MicroBrightField, Williston, VT, USA) for valuable help in implementing the Space Balls method. This work was supported by the Stanley Medical Research Institute (\#02R-258, \#04R-674 to HH, PRH, and CS), the European Community (Quality of Life and Management of Living Resources, QLK6-CT-2000-60042, QLK6-GH-0060042-46, to PK), and NIH grant MH66392 to PRH.

\section{References}

1. Andreasen NC, Rezai K, Alliger R et al (1992) Hypofrontality in neuroleptic-naive patients and in parients with chronic schizophrenia. Assessment with xenon 133 single-photon emission computed tomography and the Tower of London. Arch Gen Psychiatry 49:943-958

2. Arnold SE, Trojanowski JQ (1996) Recent advances in defining the neuropathology of schizophrenia. Acta Neuropathol 92:217-231

3. Baborie A, Kuschinksy W (2006) Lack of relationship between cellular density and either capillary density or metabolic rate in different regions of the brain. Neurosci Letr 404:20-22

4. Barch DM, Mathews JR, Buckner RL et al (2003) Hemodynamic responses in visual, motor, and somatosensory cortices in schizophrenia. NeuroImage 20:1884-1893

5. Basu S, Nagy JA, Pal S et al (2001) The neurotransmitter dopamine inhibits angiogenesis induced by vascular permeability factor/vascular endothelial growth factor. Nat Med 7:569574

6. Beckmann H, Lauer M (1997) The human striatum in schizophrenia. II. Increased number of striatal neurons in schizophrenics. Psychiatry Res 68:99-109

7. Berman KF, Zec RF, Weinberger DR (1986) Physiologic dysfunction of dorsolateral prefrontal cortex in schizophrenia. II. Role of neuroleptic treatment, attention, and mental effort. Arch Gen Psychiatry 43:126-135

8. Bogerts B (1984) Zur Neuropathologie der Schizophrenien. Fortschr Neurol Psychiatr 52:428-437

9. Braak H, Braak E (1983) Neuronal types in the basolateral amygdaloid nuclei of man. Brain Res Bull 11:349-365

10. Brambilla P, Cerini R, Fabene PF et al (2007) Assessment of cerebral blood volume in schizophrenia: A magnetic resonance imaging study. J Psychiatr Res 41:502-510

11. Brockhaus H (1942) Zur feineren Anatomie des Septum und des Striatum. J Psychol Neurol 5:1-56

12. Byne W, Buchsbaum MS, Mattiace LA et al (2002) Postmortem assessment of thalamic nuclear volumes in subjects with schizophrenia. Am J Psychiatry 159:59-65

13. Calhoun ME, Mouton PR (2000) Length measurement: new developments in neurostereology and $3 D$ imagery. J Chem Neuroanat 21:257-265

14. Casanova MF, de Zeeuw L, Switala A et al (2005) Mean cell spacing abnormalities in the neocortex of patients with schizophrenia. Psychiatry Res 133:1-12 
15. Catafau AM, Parellada E, Lomena FJ et al (1994) Prefrontal and temporal blood flow in schizophrenia: resting and activation Technetium-99m-HMPAO-SPECT patterns in young neuroleptic-naive parients with acute disease. J Nucl Med 35:935-941

16. Cohen BM, Yurgelun-Todd D, English CD et al (1995) Abnormalities of regional distribution of cerebral vasculature in schizophrenia detected by dynamic susceptibility contrast MRI. Am J Psychiatry 152:18011803

17. Cullen TJ, Walker MA, Parkinson $\mathrm{N}$ et al (2003) A postmortem study of the mediodorsal nucleus of the thalamus in schizophrenia. Schizophr Res 60:157-166

18. Danos P, Schrnidt A, Baumann B et al (2005) Volume and neuron number of the mediodorsal thalamic nucleus in schizophrenia; a replication study. Psychiatry Res 140:281289

19. Davis KL, Stewart DG, Friedman JI et al (2003) White matter changes in schizophrenia: evidence for myelin-related dysfunction. Arch Gen Psychiatry 60:443-456

20. Dewulf A (1971) Anatomy of the normal human thalamus: topometry and standardized nomenclature. Elsevier, Amsterdam

21. Dorph-Petersen KA, Pierri JN, Sun Z et al (2004) Stereological analysis of the mediodorsal thalamic nucleus in schizophrenia: volume, neuron number, and cell types. J Comp Neurol 472:449-462

22. Farkas E, Donka G, de Vos RA et al (2004) Experimental cerebral hypoperfusion induces white matter injury and microglial activation in the rat brain. Acta Neuropathol 108:57-64

23. Franzen G, Ingvar DH (1975) Absence of activation in frontal structures during psychological testing of chronic schizophrenics. J Neurol Neurosurg Psychiatry 38:1027-1032

24. Gundersen HJG (2002) Stereological estimation of tubular length. J Microsc 207:15-160

25. Hakak Y, Walker JR, Li C et al (2001) Genome-wide expression analysis reveals dysregulation of myelination-related genes in chronic schizophrenia. Proc Natl Acad Sci USA 98:4746-4751

26. Hanson DR, Gottesman II (2005) Theories of schizophrenia: a genetic-inflammatoryvascular synthesis. BMC Med Genet 6:7

27. Harrison PJ (1999) The neuropathology of schizophrenia. A critical review of the data and their interpretation. Brain 122:593-624

28. Harrison PJ, Owen MJ (2003) Genes for schizophrenia? Recent findings and their pathophysiological implications. Lancet 361:417-419

29. Harrison PJ, Weinberger DR (2005) Schizophrenia genes, gene expression, and neuropathology: on the matter of their convergence. Mol Psychiatry 10:40-68

30. Heinsen H, Heinsen YL (1991) Serial thick, frozen, gallocyanin stained sections of human central nervous system. J Histotechnol 14:167-173

31. Heinsen H, Rub U, Bauer $\mathrm{M}$ et al (1999) Nerve cell loss in the thalamic mediodorsal nucleus in Huntington's disease. Acta Neuropathol 97:613-622

32. Hill K, Mann L, Laws KR et al (2004) Hypofrontality in schizophrenia: a meta-analysis of functional imaging studies. Acta Psychiatr Scand 110:243-256

33. Hirai T, Jones EG (1989) A new parcellation of the human thalamus on the basis of histochemical staining. Brain Res Rev 14:1-34

34. Hof PR, Haroutunian V, Copland C et al (2002) Molecular and cellular evidence for an oligodendrocyte abnormality in schizophrenia. Neurochem Res 27:1193-1200 
35. Hof PR, Haroutunian V, Friedrich VL Jr et al (2003) Loss and altered spatial distribution of oligodendrocytes in the superior frontal gyrus in schizophrenia. Biol Psychiatry 53:10751085

36. Holt DJ, Herman MM, Hyde TM et al (1999) Evidence for a deficit in cholinergic interneurons in the striatum in schizophrenia. Neuroscience 94:21-31

37. Hunziker O, Schweizer A (1977) Postmortem changes in stereological parameters of cerebral capillaries. Beitr Pathol 161:244-255

38. Ingvar DH, Franzen G (1974) Distribution of cerebral activity in chronic schizophrenia. Lancet December 21:1484-1486

39. Iwamoto K, Bundo M, Kato T (2005) Altered expression of mitochondria-related genes in postmortem brains of patients with bipolar disorder or schizophrenia, as revealed by large-scale DNA microartay analysis. Hum Mol Genet 14:241-253

40. Jones EG (1997) A description of the human thalamus. In: Steriade M, Jones EG, McCormick DA (eds) Thalamus, vol. II. Experimental and clinical aspects. Elsevier Science, Oxford, pp 425-500

41. Kety SS (1959a) Biochemical theories of schizophrenia. I. Science 129:1528-1532.

42. Kety SS (1959b) Biochemical theories of schizophrenia. II. Science 129:1590-1596

43. Khaitovich P, Lockstone HE, Wayland MT et al (2008) Metabolic changes in schizophrenia and human brain evolution. Genome Biol 9: R124

44. Kim HJ, Koh PO, Kang SS et al (2001) he localization of dopamine D2 receptor mRNA in the human placenta and the anti-angiogenic effect of apomorphine in the chorioallantoic membrane. Life Sci 68:1031-1040

45. Kreczmanski P, Schmidt-Kastner R, Heinsen $\mathrm{H}$ et al (2005) Stereological studies of capillary length density in the frontal cortex of schizophrenics. Acta Neuropathol 109:510-518

46. Kreczmanski $\mathrm{P}$, Heinsen $\mathrm{H}$, Mantua $\mathrm{V}$ et al (2007) Volume, neuron density, and total neuron number in five subcortical regions in schizophrenia. Brain 130:678-692

47. Krimer LS, Muly EC 3rd, Williams GV et al. (1998) Dopaminergic regulation of cerebral cortical microcirculation. Nat Neurosci 1:286-289

48. Lauer M, Heinsen H (1996) Cytoarchitectonics of the human nucleus accumbens. J Hirnforsch 37:243-254

49. Lauer M, Beckmann $H$ (1997) The human striatum in schizophrenia. I. Increase in overall relative striatal volume in schizophrenics. Psychiatry Res 68:87-98

50. Lauer M, Senitz D, Beckmann H (2001) Increased volume of the nucleus accumbens in schizophrenia. J Neural Transm 108:645-660

51. Lewis DA, Lewitt P (2002) Schizophrenia as a disorder of neurodevelopment. Annu Rev Neurosci 25:409-432

52. Li JZ, Vawter MP, Walsh DM et al (2004) Systematic changes in gene expression in postmortem human brains associated with tissue $\mathrm{pH}$ and terminal medical conditions. Hum Mol Genet 13:609-616

53. Malaspina D, Harkavy-Friedman J, Corcoran C et al (2004) Resting neural activity distinguishes subgroups of schizophrenia patients. Biol Psychiatry 56:931-937

54. Meyer-Lindenberg A, Miletich RS, Kohn PD et al (2002) Reduced prefrontal activity predicts exaggerated striatal dopaminergic function in schizophrenia. Nat Neurosci 5:267-271

55. Middleton FA, Mirnics K, Pierri JN et al (2002) Gene expression profiling reveals alterations of specific metabolic pathways in schizophrenia. J Neurosci 22:2718-2729 
56. Mouton PR, Gokhale AM, Ward NL et al (2002) Stereological length estimation using spherical probes. J Microsc 206:54-64

57. Mueser KT, McGurk SR (2004) Schizophrenia. Lancet 363:2063-2072

58. Pakkenberg B (1990) Pronounced reduction of total neuron number in mediodorsal thalamic nucleus and nucleus accumbens in schizophrenics. Arch Gen Psychiatry 47:1023-1028

59. Popken GJ, Bunney WE, Potkin SG et al (2000) Subnucleus-specific loss of neurons in medial thalamus of schizophrenics. Proc Natl Acad Sci USA 97:9276-9280

60. Prabakaran S, Swatton JE, Ryan MM et al (2004) Mitochondrial dysfunction in schizophrenia: evidence for compromised brain metabolism and oxidative stress. Mol Psychiatry 9:684-697

61. Schmidt-Kastner R, van Os J, W M Steinbusch H et al (2006) Gene regulation by hypoxia and the neurodevelopmental origin of schizophrenia. Schizophr Res 84:253-271

62. Schmizz C, Hof PR (2005) Design-based stereology in neuroscience. Neuroscience 130:813-831

63. Schultz SK, O'Leary DS, Boles Ponto LL et al (2002) Age and regional cerebral blood flow in schizophrenia: age effects in anterior cingulate, frontal, and parietal cortex. J Neuropsychiatry Clin Neurosci 14:19-24

64. Schumann CM, Amaral DG (2005) Stereological estimation of the number of neurons in the human amygdaloid complex. J Comp Neurol 491:320-329

65. Siever LJ, Davis KL(2004) The pathophysiology of schizophrenia disorders: perspectives from the spectrum. Am J Psychiatry 161:398-413

66. Sims KS, Williams RS (1990) The human amygdaloid complex: a cytologic and histochemical atlas using Nissl, myelin, acetylcholinesterase and nicotinamide adenine dinucleotide phosphate diaphorase staining. Neuroscience 36:449-472

67. Sorvari H, Soininen H, Pitkanen A (1996) Calbindin-D28K-immunoreactive cells and fibres in the human amygdaloid complex. Neuroscience 75:421-443

68. Teunis MA, Kavelaars A, Voest E (2002) Reduced tumor growth, experimental metastasis formation, and angiogenesis in rats with a hyperreactive dopaminergic system. FASEB J 16:1465-1467

69. Tkachev D, Mimmack ML, Ryan MM et al (2003) Oligodendrocyte dysfunction in schizophrenia and bipolar disorder. Lancer 362:798-805

70. Tomita H, Vawter MP, Walsh DM et al (2004) Effect of agonal and postmortem factors on gene expression profile: quality control in microarray analyses of postmortem human brain. Biol Psychiatry 55:346-352

71. Tsuang M (2000) Schizophrenia: genes and environment. Biol Psychiat 47:210-220

72. Vawter MP, Tomita $H$, Meng $F$ et al (2006) Mitochondrial-related gene expression changes are sensitive to agonal-pH state: implications for brain disorders. Mol Psychiatry 615:663-679

73. Webster MJ, Knable MB, Johnston-Wilson $N$ et al (2001) Immunohistochemical localization of phosphorylated glial fibrillary acidic protein in the prefrontal cortex and hippocampus from patients with schizophrenia, bipolar disorder, and depression, Brain Behav Immunol 15:388-400

74. Weinberger DR, Berman KF, Zec RF (1986) Physiologic dysfunction of dorsolateral prefrontal cortex in schizophrenia. I. Regional cerebral blood flow evidence. Arch Gen Psychiatry 43:114-124

75. Young KA, Manaye KF, Liang C et al (2000) Reduced number of mediodorsal and anterior thalamic neurons in schizophrenia. Biol Psychiatry 47:944-953 


\section{Tables}

Table 1. Clinical characteristics of all cases included in this study.

\begin{tabular}{|c|c|c|c|c|c|c|c|c|c|}
\hline \multirow[t]{2}{*}{ No } & \multirow{2}{*}{$\begin{array}{c}\text { Age } \\
\text { [years] }\end{array}$} & \multirow{2}{*}{\multicolumn{3}{|c|}{ Cause of death }} & \multirow{2}{*}{$\begin{array}{c}\text { PMI } \\
\text { [hours] }\end{array}$} & \multirow{2}{*}{$\begin{array}{c}\text { Fix } \\
\text { [days] }\end{array}$} & \multicolumn{3}{|c|}{ Diagnosis } \\
\hline & & & & & & & & & ICD-10 \\
\hline$\overline{51}$ & 22 & \multicolumn{3}{|l|}{$\overline{\text { Suicide }}$} & 88 & 130 & \multicolumn{2}{|c|}{295.30} & F20.00 \\
\hline $52+$ & 36 & \multicolumn{3}{|l|}{ Suicide $\neq$} & $<72$ & 115 & \multicolumn{2}{|c|}{295.30} & $F 20.00$ \\
\hline 53 & 46 & \multicolumn{3}{|c|}{ Systemic hypothermia } & $<24$ & 327 & \multicolumn{2}{|c|}{295.30} & $F 20.01$ \\
\hline S4 & 50 & \multicolumn{3}{|l|}{ Peritonitis } & $<24$ & 203 & \multicolumn{2}{|c|}{295.30} & $F 20.00$ \\
\hline S5 & 50 & \multicolumn{3}{|l|}{ Suicide } & 18 & 170 & \multicolumn{2}{|c|}{295.30} & $F 20.00$ \\
\hline 56 & 51 & \multicolumn{3}{|l|}{ Septicemia } & 33 & 127 & \multicolumn{2}{|c|}{295.60} & $F 20.50$ \\
\hline 57 & 54 & \multicolumn{3}{|l|}{ Septicemia } & 27 & 250 & \multicolumn{2}{|c|}{295.60} & $F 20.50$ \\
\hline 58 & 55 & \multicolumn{3}{|c|}{ Right-sided heart failure } & 25 & 84 & \multicolumn{2}{|c|}{295.30} & $F 20.00$ \\
\hline S9 & 57 & Septicemia & & & 76 & 163 & & & $F 20.00$ \\
\hline 510 & 60 & Pulmonary em & olism & & $<48$ & 311 & & & $F 20.01$ \\
\hline $\mathrm{S} 11$ & 62 & Aspiration & & & 7 & 171 & & & $F 20.00$ \\
\hline $\mathrm{S12}$ & 63 & Acute myocar & al infarct & & 15 & 338 & & & $\mathrm{~F} 20.50$ \\
\hline$\$ 13$ & 64 & Pulmonary em & olism & & 6 & 817 & & & $\mathrm{~F} 20.50$ \\
\hline $\mathrm{C} 1+$ & 25 & Cardiac tampc & ade & & 14 & 119 & & & - \\
\hline $\mathrm{C} 2 \mathrm{t}$ & 36 & Gunshot & & & 24 & 143 & & & - \\
\hline C3 & 47 & Acute myocar & al infarct & & $<24$ & 133 & & & - \\
\hline$C_{4}$ & 50 & Acute myocar & al infarct & & 35 & 433 & & & - \\
\hline $\mathrm{C} 5$ & 50 & Avalanche acc & ent & & 23 & 498 & & & - \\
\hline $\mathrm{C} 6 \mathrm{t}$ & 51 & Septicemia & & & 7 & 285 & & & - \\
\hline C7 & 54 & Acute myocar & al infarct & & 18 & 168 & & & - \\
\hline $\mathrm{C} 8$ & 56 & Acute myocar & al infarct & & 60 & 3570 & & & - \\
\hline C9 & 58 & Acute myocar & al infarct & & 28 & 126 & & & - \\
\hline $\mathrm{C} 10$ & 60 & Gastrointestin & hemorrh & & 18 & 101 & & & - \\
\hline $\mathrm{C} 11$ & 60 & Gastrointestin & hemorrt & & 27 & 302 & & & - \\
\hline $\mathrm{Cl} 2+$ & 62 & Acute myocar & al infarct & & $<24$ & 3696 & & & - \\
\hline $\mathrm{C} 13$ & 65 & Bronchopneu & onia & & 6 & 2289 & & & - \\
\hline & Varia & & $\begin{array}{l}\text { Brain } \\
\text { region }\end{array}$ & $A / I D$ & PMI & Fix & D & H & $\mathrm{D} \times \mathrm{H}$ \\
\hline & & & $\overline{C N}$ & 0.399 & 0.977 & 0.990 & 0.034 & 0.434 & 0.838 \\
\hline Micro & essel leng & h density & PNA & 0.926 & 0.509 & 0.605 & 0.077 & 0.101 & 0.727 \\
\hline & & & & 0.434 & 0.320 & 0.842 & 0.231 & 0.863 & 0.738 \\
\hline & & & MDNT & 0.991 & 0.697 & 0.251 & 0.034 & 0.541 & 0.719 \\
\hline & & & LNA & 0.129 & 0.844 & 0.709 & 0.607 & 0.426 & 0.057 \\
\hline & & & $C N$ & 0.276 & 0.788 & 0.436 & 0.312 & 0.273 & 0.998 \\
\hline Total & icrovesse & length & PNA & 0.697 & 0.034 & 0.342 & 0.409 & 0.417 & 0.833 \\
\hline & & & & 0.236 & 0.949 & 0.381 & 0.839 & 0.512 & 0.850 \\
\hline & & & MDNT & 0.099 & 0.825 & 0.035 & 0.951 & 0.209 & 0.419 \\
\hline & & & LNA & 0.446 & 0.661 & 0.403 & 0.015 & 0.942 & 0.837 \\
\hline & & & $C N$ & 0.104 & 0.328 & 0.592 & 0.225 & 0.427 & 0.751 \\
\hline Micro & essel leng & h per neuron & PNA & 0.434 & 0.670 & 0.677 & 0.086 & 0.578 & 0.623 \\
\hline & & & MDNT & 0.075 & 0.130 & 0.280 & 0.391 & 0.095 & 0.682 \\
\hline & & & & 0.052 & 0.621 & 0.052 & 0.645 & 0.849 & 0.926 \\
\hline & & & LNA & 0.677 & 0.782 & 0.365 & 0.870 & 0.378 & 0.969 \\
\hline
\end{tabular}

S, patient with schizophrenia; $C$, control. PMl, postmortem interval (time between death and autopsy); Fix, fixation time. tThe microvessel length densities in the left and right hemispheres of the cases $C_{1}$ and $C_{2}$ (as well 
as in the right hemispheres in $\mathrm{C} 6, \mathrm{C} 12$ and $\$ 2$, respectively) could not be determined because the corresponding sections not stained with gallocyanin were missing. ¥These two patients had relatively long postmortem intervals. However, both had committed suicide (one by hanging, the other by jumping from a building), were found within one hour of death and were kept at $4^{\circ} \mathrm{C}$ until autopsy. Accordingly, the postmortem intervals between death and autopsy of these patients cannot be compared to the corresponding intervals of the other cases and were thus excluded from the calculation of the mean postmortem intervals.

Table 2. Details of the stereologic procedure to measure microvessel length densities with the Space Balls method.

\begin{tabular}{cccccc} 
& CN & P & NA & MDNT & LNA \\
\hline Obj. & $40 x$ & $40 x$ & $40 x$ & $40 x$ & $40 x$ \\
$r(\mu \mathrm{m})$ & 30 & 30 & 30 & 30 & 30 \\
$t[\mu \mathrm{m}]$ & 45.6 & 45.7 & 45.6 & 46.2 & 45.5 \\
D $(\mu \mathrm{m})$ & 725 & 925 & 550 & 600 & 400 \\
$\Sigma$ hs & 388 & 398 & 390 & 396 & 497 \\
$\Sigma$ is & 500 & 550 & 376 & 507 & 374 \\
\hline
\end{tabular}

CN, caudate nucleus; $P$, putamen; NA, nucleus accumbens; MDNT, mediodorsal nucleus of the thalamus; LNA, lateral nucleus of the amygdala. Obj., objective used; $r$, radius of the hemispheres; $D$, distance between the midpoints of the centers of the hemispheres in directions $X$ and $Y_{;} \Sigma$ hs, total number of hemispheres used; $\Sigma$ is, total number of intersections between the hemispheres and the capillaries.

Table 3. Results of statistical analysis ( $p$ values) with generalized linear model multivariate analysis of variance (MANOVA).

\begin{tabular}{clllllll}
\multicolumn{1}{c}{ Variable } & $\begin{array}{c}\text { Brain } \\
\text { region }\end{array}$ & A/10 & PMI & Fix & D & H & DxH \\
\hline \multirow{5}{*}{ Microvessel length density } & CN & 0,339 & 0,977 & 0,990 & 0,034 & 0,434 & 0,838 \\
& P & 0,926 & 0,509 & 0,605 & 0,077 & 0,101 & 0,727 \\
& NA & 0,434 & 0,320 & 0,842 & 0,231 & 0,863 & 0,738 \\
& MDNT & 0,991 & 0,967 & 0,251 & 0,034 & 0,541 & 0,719 \\
& LNA & 0,129 & 0,844 & 0,709 & 0,607 & 0,426 & 0,057 \\
Total microvessel length & CN & 0,276 & 0,788 & 0,436 & 0,312 & 0,273 & 0,998 \\
& P & 0,697 & 0,034 & 0,342 & 0,409 & 0,417 & 0,833 \\
& NA & 0,239 & 0,949 & 0,381 & 0,839 & 0,512 & 0,850 \\
& MDNT & 0,099 & 0,825 & 0,035 & 0,951 & 0,209 & 0,419 \\
Mlcrovessel length per neuron & LNA & 0,446 & 0,661 & 0,403 & 0,015 & 0,942 & 0,837 \\
& CN & 0,104 & 0,328 & 0,592 & 0,645 & 0,849 & 0,751 \\
& P & 0,434 & 0,670 & 0,677 & 0,086 & 0,578 & 0,623 \\
& NA & 0,075 & 0,130 & 0,280 & 0,391 & 0,095 & 0,682 \\
& MDNT & 0,052 & 0,621 & 0,052 & 0,645 & 0,849 & 0,926 \\
& LNA & 0,677 & 0,782 & 0,365 & 0,870 & 0,378 & 0,969 \\
\hline
\end{tabular}

$C N$, caudate nucleus; $P$, putamen; NA, nucleus accumbens; MDNT, mediodorsal nucleus of the thalamus; LNA, lateral nucleus of the amygdala. AVID, age (controls) or adjusted illiness duration (patients with schizophrenia), respectively (adjusted illness duration calculated as age at death minus age at onset plus mean age at onset; details are provided in Statistical analysis); PMI, postmortem interval (time between death and autopsy); Fix, fixation time; $D$, diagnosis; $H$, hemisphere. $P$ values smaller than 0.05 are shown in boldface. 
Figures

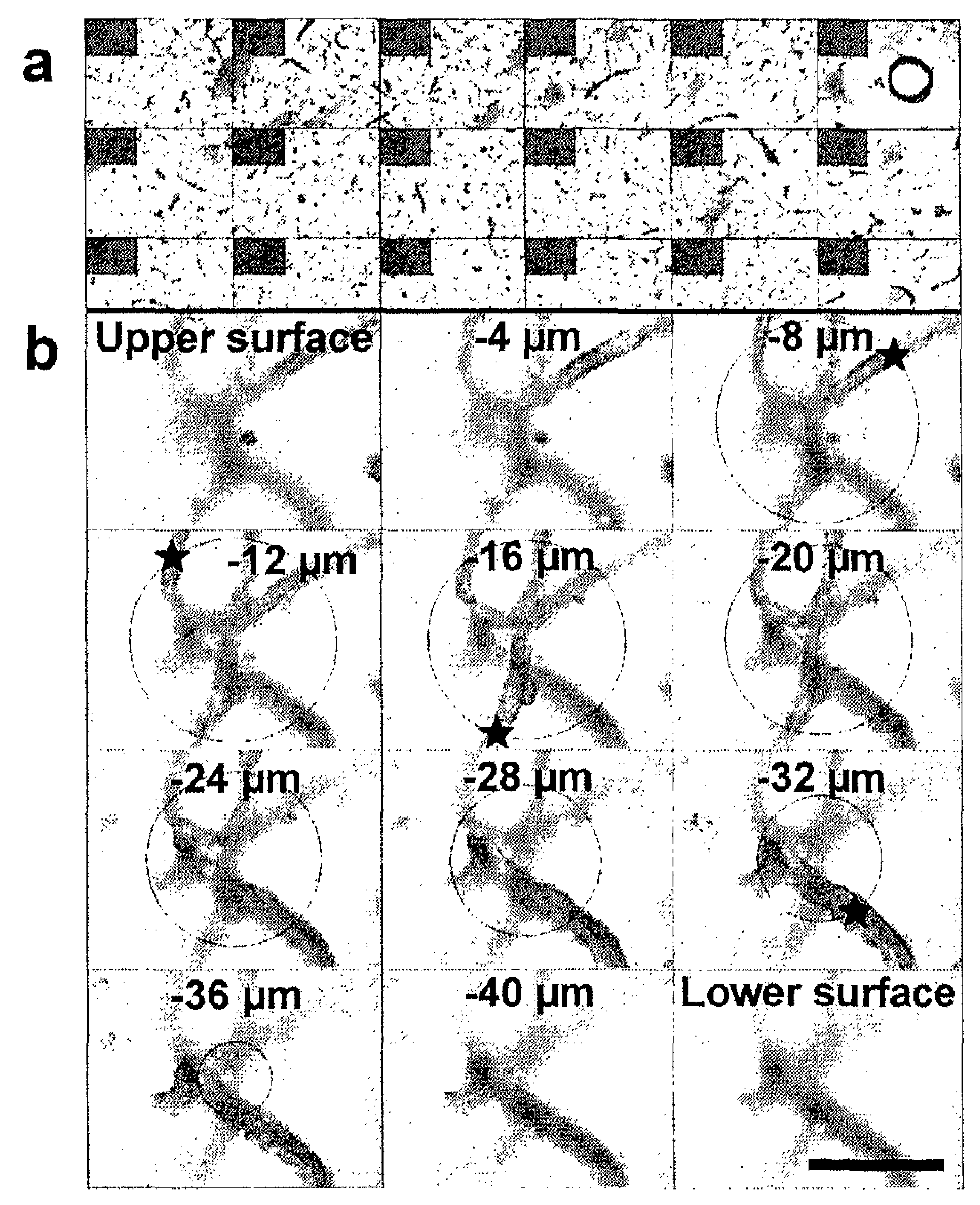

Fig. 1. Estimating microvessel length density with the Space Balls method, illustrated for the caudate nucleus of control brain no. C4. (a), Low-power overview of capillaries, superimposed by a rectangular lattice with uniform distance between the lines in the $X$ and $Y$ axes. This lattice defines the position of microscopic fields (indicated as gray rectangles), at which the section is inspected at higher magnification to perform Space Ball analyses. (b), High-power photomicrographs of one of these microscopic fields at the upper surface of the section, at 10 consecutive focal planes below the upper surface with a distance of $4 \mu \mathrm{m}$ between the focal planes, and at the lower surface of the section that was found $43 \mu \mathrm{m}$ below the upper surface. Between $-8 \mu \mathrm{m}$ and $-36 \mu \mathrm{m}$ the microscopic field is superimposed by intersections of a hemisphere (a semi-Space Ball) with the focal plane at the investigated focal depth, illustrated as circles. The semi-space ball was centered at a depth of $-8 \mu \mathrm{m}$ and had a radius of $30 \mu \mathrm{m}$. Intersections between the semi-Space Ball and the microvessels in focus were found at $-12 \mu \mathrm{m},-16 \mu \mathrm{m}$, $-24 \mu \mathrm{m}$ and $-28 \mu \mathrm{m}$ (asterisks). The average microvessel length density within the caudate nucleus was calculated from the total number of intersections and the number and size of the semi-space balls. Scale bar: $700 \mu \mathrm{m}$ in $\mathrm{A}$, and $40 \mu \mathrm{m}$ in the high-power photomicrographs. 


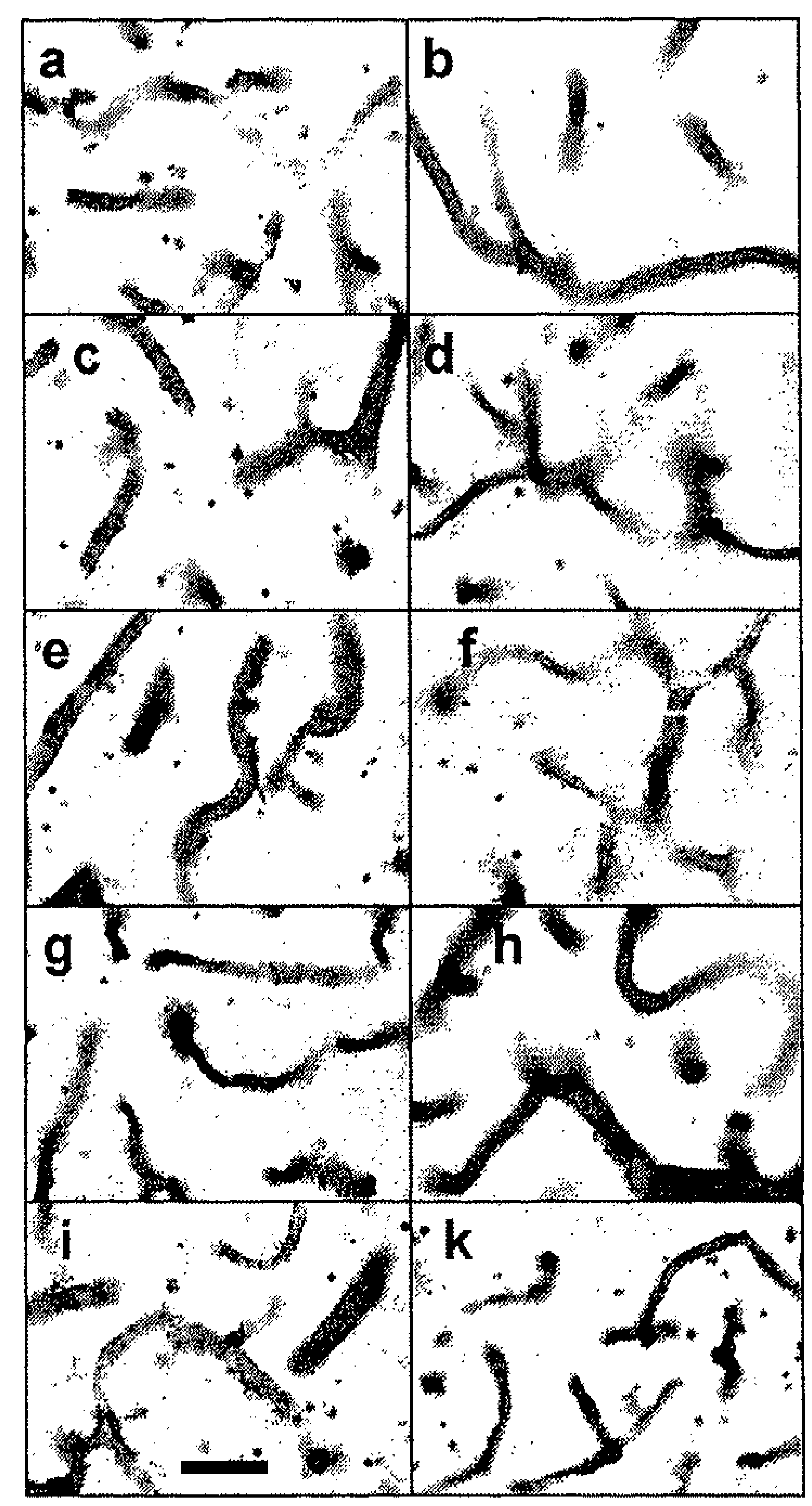

Fig. 2. Representative high-power photomicrographs of $50 \mu$ m-thick coronal sections from the left brain half of a patient with schizophrenia $(a, c, e, g, i)$ and a control $(b, d, f, h, k)$ showing immunohistochemical detection of collagen $\mathrm{V}$ in the caudate nucleus $(A, B)$, putamen $(c, d)$, nucleus accumbens $(e, f)$, mediodorsal nucleus of the thalamus $(g, h)$ and lateral nucleus of the amygdala $(i, k)$. Note that no major abnormalities such as irregular size of the vascular wall, stenosis or dilation were noted in the immunohistochemical preparations for collagen $\mathrm{IV}$ in the materials from the patients with schizophrenia. Furthermore, differences in microvessel length density between patients with schizophrenia and controls were not obvious at simple observation of the tissue but necessitated the type of rigorous quantitative analysis undertaken in this study to be revealed. Scale bar: $50 \mu \mathrm{m}$. 


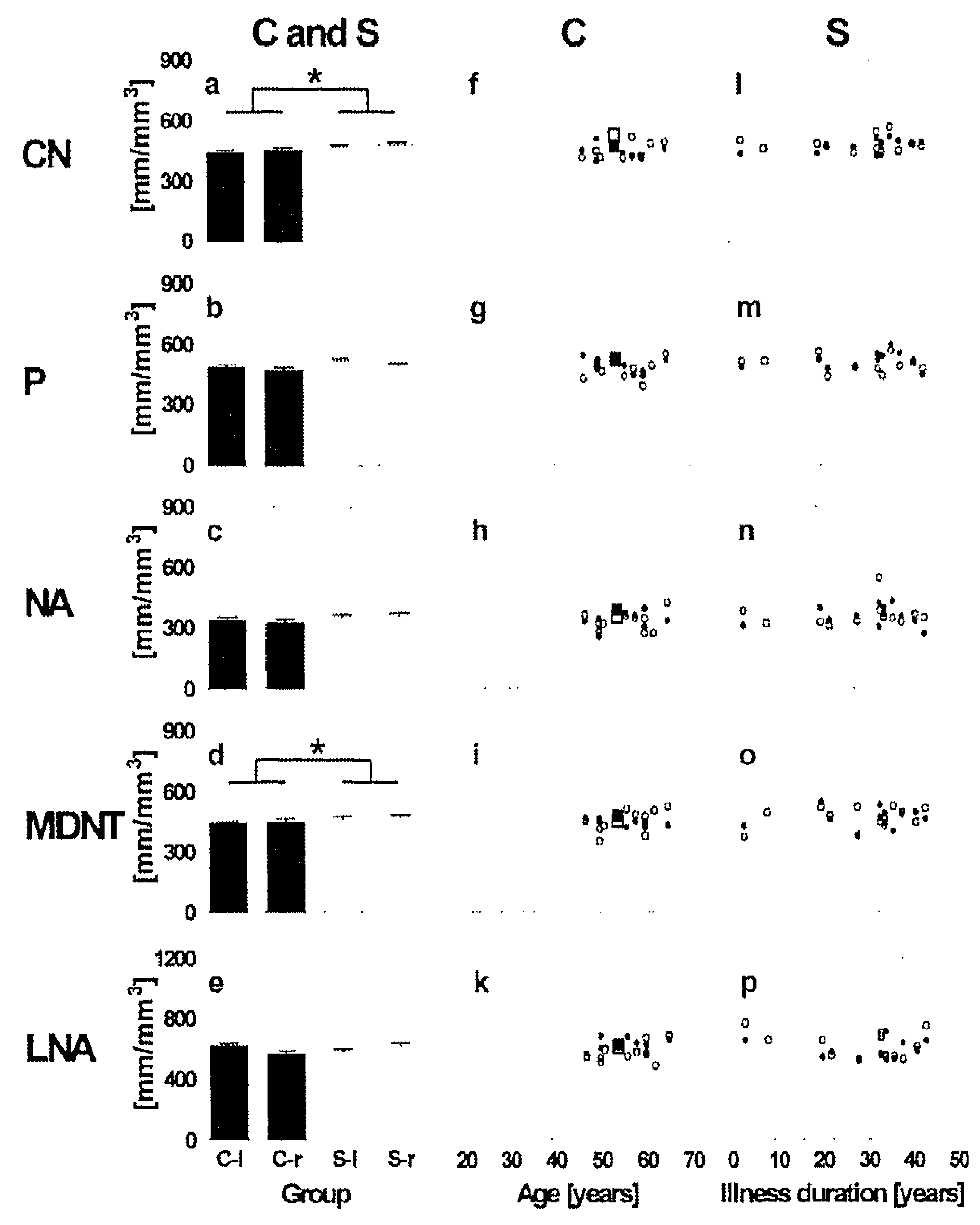

Fig. 3. Microvessel length density in caudate nucleus $(C N ; a, f, l)$, putamen $(P ; b, g, m)$, nucleus accumbens (NA; $\left.c_{1} h, n\right)$, mediodorsal nucleus of the thalamus (MDNT; $d, i, 0$ ) and lateral nucleus of the amygdala ( $(L N A ; e, k, p)$ in both hemispheres of the brains from 13 male patients with schizophrenia (S; open bars in a to e, and dots in $f$ to $p$ ) and 13 age-matched controls $\left(C_{;}\right.$closed bars in a to $e_{\text {, }}$ and dots in $f$ to $p$; squares in case of the results obtained for the single brain embedded in celloidin [C7]). In a to e, data are shown as mean and standard error of the mean for the left (I) and right $(r)$ hemispheres from patients with schizophrenia ( $\mathrm{S}-\mathrm{l}$ and $\mathrm{S}-\mathrm{r}$ ) and controls $(\mathrm{C}-\mathrm{l}$ and $\mathrm{C}-\mathrm{r}$ ). In $\mathrm{f}$ to $p$, individual data for the left hemispheres (closed dots or squares, respectively) and right hemispheres (open dots or squares, respectively) from controls ( $\mathrm{fto} k$ ) and patients with schizophrenia $(l$ to $p$ ) are shown as function of the control patients' age (or the illness duration of the patients with schizophrenia, respectively). ${ }^{*}, p<0.05$ for the fixed factor Diagnosis in general linear model multivariate analysis of variance (MANOVA). 


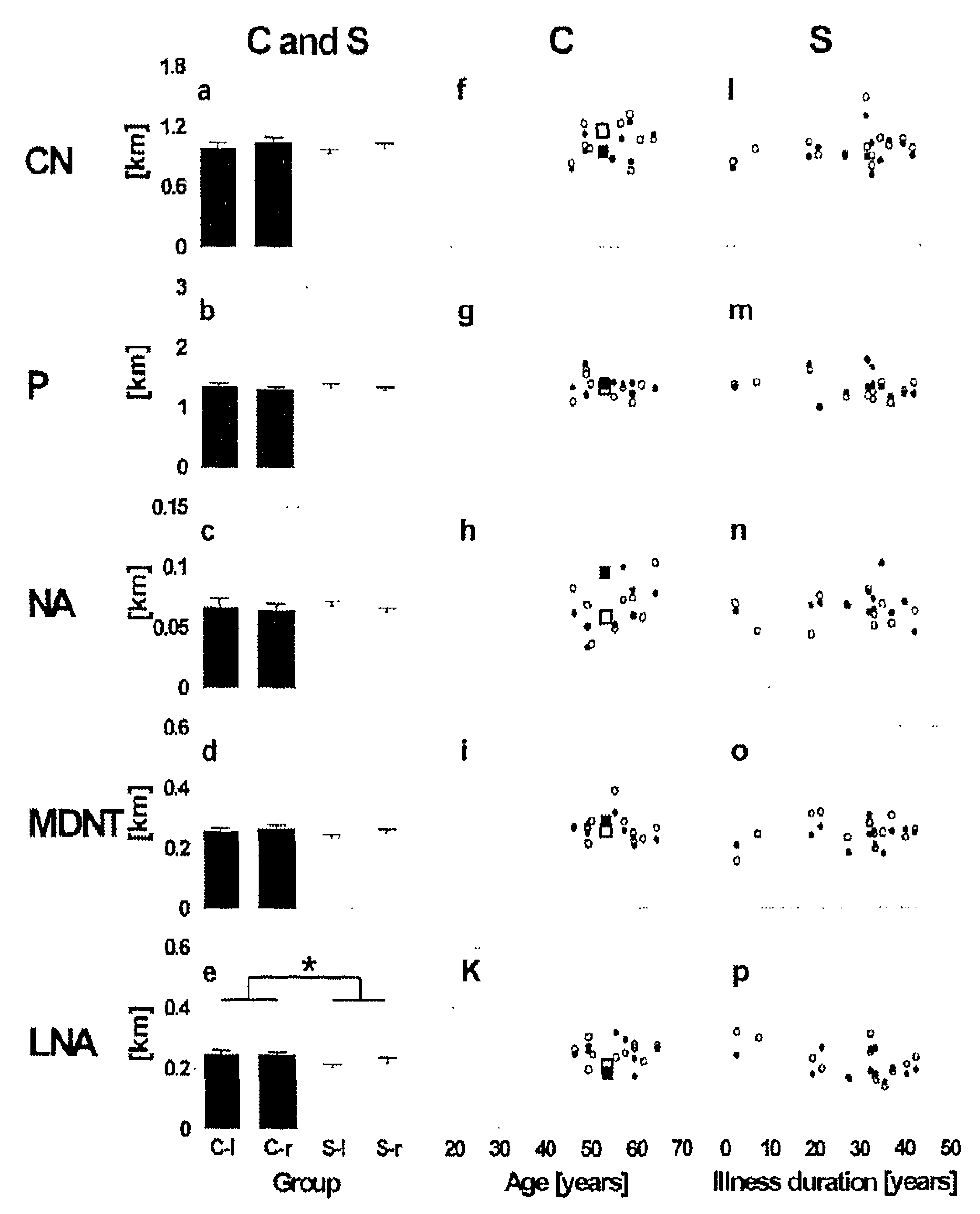

Fig. 4. Microvessel length in caudate nucleus $(C N ; a, f, l)$, putamen $(P ; b, g, m)$, nucleus accumbens $(N A ; c, h, n)$, mediodorsal nucleus of the thalamus (MDNT; $d, i, O)$ and lateral nucleus of the amygdala (LNA; $e, k, p)$ in both hemispheres of the brains from 13 male patients with schizophrenia ( $S$; open

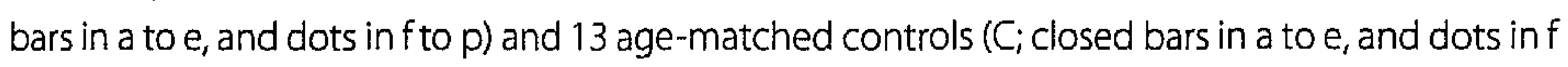
to $p$; squares in case of the results obtained for the single brain embedded in celloidin [C7]). In a to $e_{1}$ data are shown as mean and standard error of the mean for the left ( () and right $(r)$ hemispheres from patients with schizophrenia (S-I and S-r) and controls $(C-I$ and $C-r)$. In $f$ to $p$, individual data for the left hemispheres (closed dots or squares, respectively) and right hemispheres (open dots or squares, respectively) from controls ( $f$ to $k$ ) and patients with schizophrenia ( to $p$ ) are shown as function of the control patients' age (or the illness duration of the patients with schizophrenia, respectively). ${ }^{*}, p<0.05$ for the fixed factor Diagnosis in general linear model multivariate analysis of variance (MANOVA). 


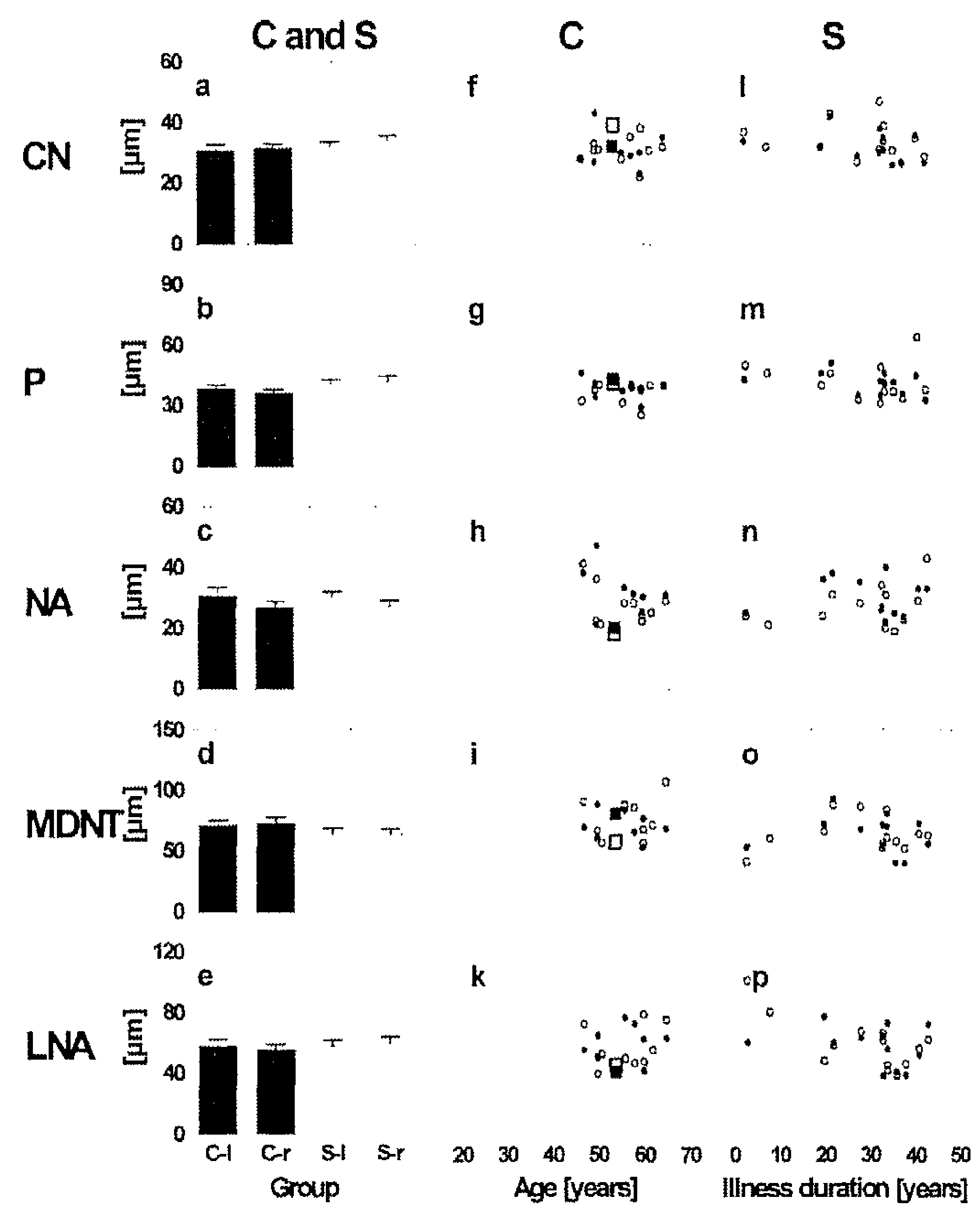

Fig. 5. Microvessel length per neuron in caudate nucleus $(C N ; a, f$,$) , putamen (P ; b, g, m)$, nucleus accumbens $(N A ; c, h, n)$, mediodorsal nucleus of the thalamus (MDNT; di,o) and lateral nucleus of the amygdala $(\mathrm{LNA} ; \mathrm{e}, \mathrm{k}, \mathrm{p})$ in both hemispheres of the brains from 13 male patients with schizophrenia ( $S$; open bars in a to $e$, and dots in $f$ to $p$ ) and 13 age-matched controls $(C$; closed bars in

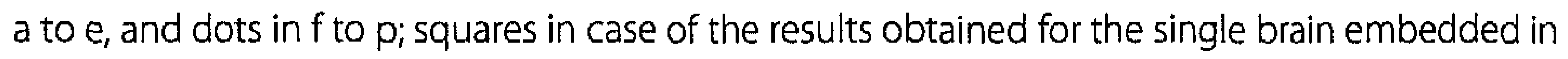
celloidin [C7]). In a to e, data are shown as mean and standard error of the mean for the left (I) and right ( $r$ ) hemispheres from patients with schizophrenia (S-l and S-r) and controls (C-I and $C-r)$. In $f$ to $\mathrm{p}$, individual data for the left hemispheres (closed dots or squares, respectively) and right hemispheres (open dots or squares, respectively) from controls ( $f$ to $k$ ) and patients with schizophrenia (l to p) are shown as function of the control patients' age (or the illness duration of the patients with schizophrenia, respectively). 


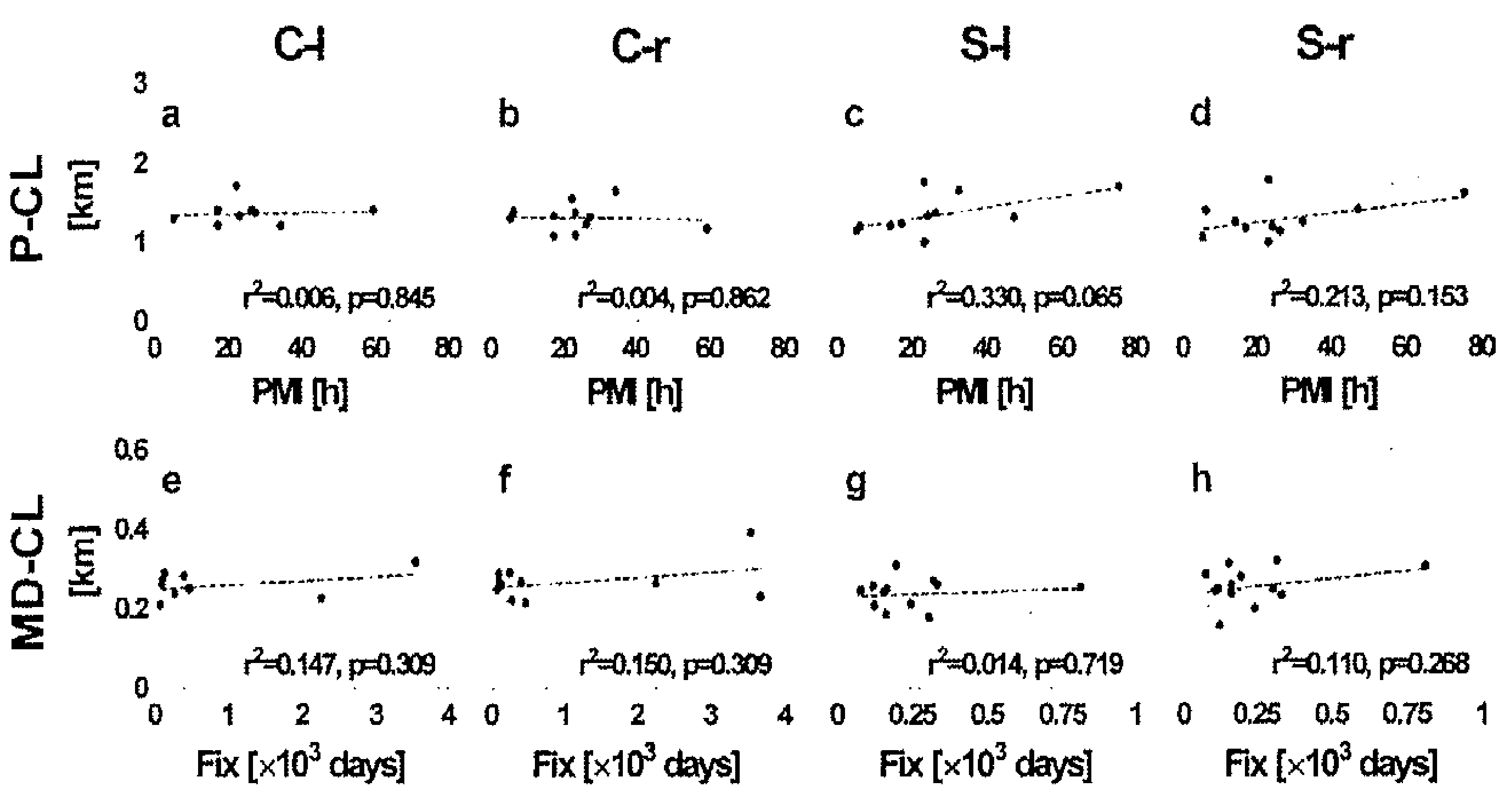

Fig. 6. Microvessel length in the putamen as a function of the postmortem interval (PMl; time between death and autopsy) ( $\mathrm{P}-\mathrm{CL} ; \mathrm{a}$ to $\mathrm{d}$ ) and microvessel length in the mediodorsal nucleus of the thalamus as a function of the fixation time (MDNT-CL; e to $h$ ). The graphs show individual values (dots) for the left $(l)$ and right $(r)$ hemispheres from controls $(C-1 ; a, e$, and $C-r ; b, f$, respectively) and patients with schizophrenia ( $\mathrm{S}-\mathrm{l} ; \mathrm{c}, \mathrm{g}$, and $\mathrm{S}-\mathrm{r} ; \mathrm{d}, \mathrm{h}$, respectively) as well as results from linear regression analysis (regression lines with 95\% confidence intervals, regression correlation coefficients [ $r 2$ values] and $p$ values). All regression lines are dotted because their slope did not significantly differ from zero $(p>0.05)$.
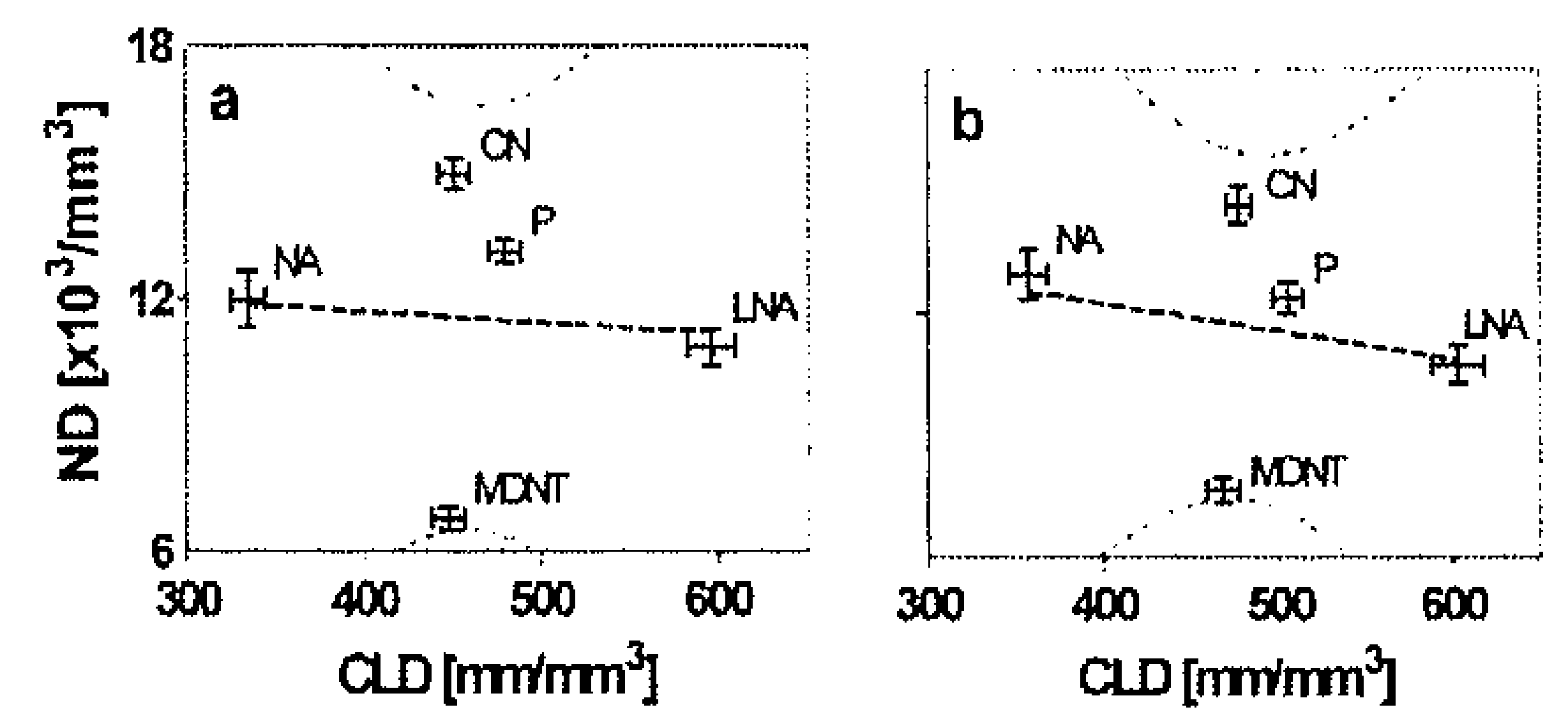

Fig. 7. Neuron density (ND) in the caudate nucleus (CN), putamen (P), nucleus accumbens (NA), mediodorsal nucleus of the thalamus (MDNT) and lateral nucleus of the amygdala (LNA) as a function of microvessel length density (CLD) in these subcortical regions in both hemispheres of postmortem brains from 13 male controls (a) and 13 male patients with schizophrenia (b). Regionspecific values for microvessel length density and neuron density are shown as mean \pm standard error of the mean (SEM). Linear regression analysis showed no significant correlation between the microvessel length density and the neuron density in these subcortical regions (controls: $r 2=0.006 ; p=0.900 ;$ patients with schizophrenia: $r 2=0.054 ; p=0.708$ ). The regression lines and their $95 \%$ confidence intervals are also shown in the graphs. 


\section{cuarre 4}

\section{Stereological studies of capillary length density in the frontal cortex of schizophrenics}

Pawel Kreczmanski' ${ }^{1}$, Rainald Schmidt-Kastner ${ }^{2}$, Helmut Heinsen ${ }^{3}$ Harry W. M. Steinbusch', Patrick R. Hof', Christoph Schmitz'

'European Graduate School of Neuroscience (EURON), Maastricht, The Netherlands 2Department of Neurology, University of Miami School of Medicine, Miaml, Florida, USA

${ }^{3}$ Morphologic Brain Research Unit, University of Wuerzburg, Wuerzburg, Germany 4Department of Neuroscience and Department of Geriatrics and Adult Development, Mount Sinai School of Medicine, New York, New York, USA 


\section{Abstract}

The presence of microvasculature abnormalities in the prefrontal cortex of schizophrenics was proposed in a recent study of molecular signatures of schizophrenia [Prabakaran et al (2004) Mol Psychiat 9:684-697]. To assess this possibility further, we investigated capillary length densities in prefrontal cortex area 9 and anterior cingulate cortex area 24 in post-mortem brains from 13 schizophrenics and 13 age- and sex-matched controls. To check that our sample of brains shared cardinal neuropathological features of schizophrenia with previously reported case studies, we also measured cortical gray matter volumes and cortical thickness in areas 9 and 24. The mean cortical gray matter volume was significantly reduced in brains from schizophrenics compared to controls. Mean cortical thickness was significantly reduced in area 24 , but not in area 9, in schizophrenics. There were no differences in mean capillary length densities in either area 9 or 24 between the two groups. Thus, alterations in capillary length density in the prefrontal cortex cannot be considered a general feature of schizophrenia. Compromised brain metabolism and occurrence of oxidative stress in the brain of schizophrenics are likely caused by other mechanisms. 


\section{Introduction}

Schizophrenia is a mental illness characterized by psychosis, cognitive impairment and social withdrawal [37]. Among psychiatric disorders, schizophrenia is the most disabling [37]. A combination of genetic susceptibility and environmental perturbations appears to be necessary for the expression of the disease $[19,48,51]$. However, the actual mechanisms involved in the pathogenesis of schizophrenia remain elusive $[18,19,51]$. Considerations range from alterations of highly specific developmental processes at the synaptic level $[17,31]$ and abnormalities in myelination $[16,50]$ to global metabolic changes of brain function $[1,3,35]$.

Anatomic and pathological analyses of brain tissue from schizophrenic patients have focused on neurons, including detailed studies of dendrites and synapses [17]. The present consensus is that a slight reduction of overall cortical volume is caused by a reduction of the neuropil that may be related to some form of abnormal developmental process for synaptic connections [46]. Another possibility is that a progressive loss of synapses occurs due to a degenerative process during the chronic course of the disease [32]. Studies of glial cells provided no evidence for reactive gliosis [13]. However, some deficits of glial cells have recently been detected in the white matter using quantitative methods [12, $25,26]$. Given the possible involvement of glial cells in pathogenetic scenarios of psychiatric disorders [12, 49], it is timely to consider the brain microvasculature as another key factor in the development of schizophrenia. As the function of neurons depends on adequate oxygen and substrate supply, subtle changes in vasculature integrity may also be important for the schizophrenic brain. General observations on the macrovasculature in the brain of schizophrenics have been made [2], but there have been no systematic studies on the vascular system in this disease. It has been argued in earlier studies that a subtle metabolic deficit, such as hypoxia, would be sufficient to cause widespread neuronal dysfunction $[28,29]$. However, reports on alterations of regional cerebral blood flow in the frontal cortex of schizophrenics under resting conditions have yielded inconsistent results $[8,33,45]$. A number of abnormalities in the coupling be- tween neuronal activity and cerebral blood flow have been described in schizophrenic patients $[24,34]$. These changes were attributed to primary neuronal dysfunc- tion, although subtle changes in the capillary system have not been excluded. Some imaging studies pointed to an abnormal cerebral vascular volume in schizo- phrenic patients [10]. Furthermore, abnormalities in the capillary system of the fingernail folds have been found in patients affected by the disorder [11], and the vasod-ilatory response to methylnicotinate application in the skin is reduced in schizophrenics [42]. A very recent study employing a combined transcriptomics, proteo- mics and metabolomics approach found evidence for mitochondrial dysfunction, compromised brain metab- olism, and oxidative stress in the frontal cortex of schizophrenics [39], thereby confirming and expanding earlier reports $[1,3$, 35]. Importantly, these authors hypothesized that these alterations can be explained by microvasculature abnormalities in the frontal cortex in schizophrenia [39]. 
We thus decided to investigate whether the brain microvasculature in the schizophrenic brain is normal. In the present study we examined, for the first time, capillary length densities within the prefrontal cortex (area 9) and anterior cingulate cortex (area 24) in post- mortem brains from schizophrenics and controls with state-of-the-art design-based stereological technology, i.e., the recently developed "Space balls" method $[7,36,44]$.

\section{Materials and methods}

\section{Brain specimens}

Postmortem brains (both hemispheres) from 13 male schizophrenics (mean age $51.5 \pm 3.3$ years; mean interval between death and autopsy $27.5 \pm 6.0 \mathrm{~h}$; data given as mean \pm SEM) and from 13 age-matched male controls (mean age $51.9 \pm 3.1$ years; mean interval between death and autopsy $23.7 \pm 3.8 \mathrm{~h}$ ) were analyzed. Clinical diag- noses, causes of death, the fixation time and the interval between death and autopsy are summarized in Table 1.

Schizophrenics did not differ from controls with respect to mean age (Student's two-tailed t-test; $\mathrm{P}=0.946)$, mean interval between death and autopsy $(\mathrm{P}=0.581)$ and mean fixation time $(\mathrm{P}=0.089)$. All schizophrenics had been patients either in German university hospitals or German State psychiatric hospitals and full clinical records were available. Records from autopsy (including a summary of the medical history) were also available for all controls. All schizophrenics met the Diagnostic Statistical Manual, 4th revision (DSM-IV) and Inter- national Statistical Classification of Diseases and Related Health Problems, 10th revision (ICD-10) diagnostic criteria. The clinical notes were assessed by two experienced clinical psychiatrists to ensure that the control brains were free from psychopathology, and for clear evidence that the schizophrenic cases conformed to DSM-IV criteria for schizophrenia. Exclusion criteria for both schizophrenics and controls comprised neurological problems that required intervention or interfered with cognitive assessment (e.g., stroke with aphasia), history of recurrent seizure disorder, history of severe head injury with loss of consciousness, diabetes mellitus with free plasma glucose $>200 \mathrm{mg} / 100 \mathrm{ml}$, and history of self-administered intoxication. Brains from schizophrenics were also excluded if patients had undergone a leukotomy. The two groups were similar in terms of the ethnic backgrounds of the subjects. However, they were not fully matched for socio-economical status and education, which would have placed severe constraints on our sample. Moreover, all schizophrenics were subjected to long-term treatment with high doses of neuroleptics. In all of the cases, autopsy was performed after consent was obtained from a relative, according to the laws of the Federal Republic of Germany. The use of these autopsy cases for scientific investigations as outlined here has been approved by the relevant Institutional Review Boards. Brains were fixed by immersion in $10 \%$ formalin ( 1 part commercial $40 \%$ aqueous formaldehyde in 9 parts $\mathrm{H}_{2} \mathrm{O}$ ) prior to histological processing. 


\section{Tissue processing}

The brainstem with the cerebellum was severed at the level of the rostral pons, and the hemispheres were divided medio-sagittally. Both hemispheres were then cut into serial 600 to 700-lm-thick coronal sections as previously described [20]. Briefly, the hemispheres were cryoprotected in a mixture of glycerol-dimethylsulfoxide-formalin after careful removing of the meninges and the pial vessels, embedded in gelatin, deeply frozen at $-60^{\circ} \mathrm{C}$, and serially sectioned using a cryomicrotome (Jung, Nussloch, Germany). One brain (control case C7) was embedded in celloidin as recently described [23], and was cut into serial 440-1m-thick coronal sections using a sliding microtome (Polycut, Cambridge Instruments, UK). From each hemisphere, every second or third section was stained with gallocyanin as described [20]. The remaining sections were stored in formalin for up to 10 years. From all brains of schizophrenics and controls older than 40 years, sections through the central portion of the entorhinal and transentorhinal cortex that were not stained with gallocyanin were labeled with the Gallyas method to detect neurofibrillary changes [21]. Neurofibrillary tangles were very rarely detected in the transentorhinal and entorhinal cortex on Gallyas- stained sections, compatible with Braak's stage I [5]. Furthermore, each section was coded and controlled for the absence of tumors, infarcts, heterotopias, signs of autolysis, staining artifacts, and gliosis.

\section{Immunohistochemistry}

From each brain and hemisphere, the 600- to 700-lm- thick coronal section stained with gallocyanin that showed the rostral tip of the caudate nucleus was selected. The adjacent caudal section not stained with gallocyanin was selected, and an approximately $4.4-\mathrm{cm}$ piece containing the cingulate gyrus and the superior frontal gyrus was cut out to investigate area 24 (the appearance of area 24 at this rostrocaudal level is de- picted in Fig. $1 \mathrm{E}$ in [14]). Another approximately $4.4 \mathrm{~cm}$ piece containing both superior frontal gyrus and medial frontal gyrus was cut out to investigate area 9. After rinsing in tap water for several hours, these pieces were stored in TRIS-buffered saline (TBS) overnight ( $\mathrm{pH} \mathrm{9.0)} \mathrm{at} \mathrm{room}$ temperature. They were then placed in TBS ( $\mathrm{pH} 9.0$ ) and heated in a microwave oven for

$15 \mathrm{~min}$ at $90^{\circ} \mathrm{C}$ for antigen retrieval. After cooling, tissue pieces were rinsed twice in TBS (pH 7.4) and were placed in TBS-based 30\% sucrose for cryoprotection. Pieces were then frozen and cut into 50-lm-thick coronal sections on a cryostat (Type HM 500 OMV, Microm, Walldorf, Germany). One 50-lm-thick section per piece was mounted on a glass slide, dried, and stained with cresyl violet $(0.01 \%, 25 \mathrm{~min})$. Slides were dehydrated and coverslipped using DePeX (Serva, Heidel- berg, Germany). These 50-lm-thick sections stained with cresyl violet as well as the adjacent 600 - to 700 -lm-thick sections stained with gallocyanin were used to identify areas 9 and 24 in both cerebral hemispheres according to cytoarchitectural criteria established in the literature.

With respect to area 9, Rajkowska and Goldman- Rakic [41] showed that lateral parts of area 9 surround area 46 (area FDD of von Economo and Koskinas [54]). It is important to note that Brodmann [6] never de-scribed the cytoarchitectural characteristics of this area in the human brain. Rather, he projected his observations in monkey onto the human frontal 
lobe. Von Economo and Koskinas [54] provided a detailed cytoarchitectonic description of the human frontal lobe. Their area FDm corresponds to area 9. Its characteris- tics are a distinct layer II, absence of very large, slender and well-built pyramidal cells in layer IIIc (typical of area 6 and to a lesser extend of area 8), and an uninterrupted layer IV with fairly definite upper and lower boundaries. In thick gallocyanin-stained sections, a cell-poor layer is found between layers IIIc and layer IV. Layer IV is more prominent and cell rich in the frontopolar regions; however, the transition between areas 9 and 10 is gradual. Layer $V$ of area 9 is subdivided into layers $\mathrm{Va}$ and $\mathrm{Vb}$, the latter being rather sparse in cells. Most probably the cell-poor layers between layer IIIc and IV and within layer Vb correspond to the upper and lower bands described by Rajkowska and Goldman- Rakic [40] in myelin-stained sections. Finally, layer VI consists of layers VIa and VIb. Layer Vla is rich in cells and its upper border abutting the cell-sparse layer $\mathrm{Vb}$ can be easily recognized. The deeper border of layer VIb with the underlying white matter are much more distinct than in the caudally located areas 8 and 6 . The overall cortical thickness of the frontopolar area 10 (field FE of von Economo and Koskinas [54]) is considerably less than in area 9, layer VIa is small, rich in darkly stained pyramidal as well as spindle-shape cells, and its boundary with the medullary layer is even more distinct than in area 9. The presence of the small and cell-rich layer VI facilitates the delineation of area 9 from the rostral area 10 in thick gallocyanin stained sections. Finally, layer IV is more conspicuous in area 10 than in area 9. Area 24 (including subfields 24a,b,c) was identified according to the criteria of Vogt et al. [53] and Nimchinsky et al. [38].

The remaining $50 \mathrm{~lm}$-thick sections were stored in TBS ( $\mathrm{pH} 7.4$ ) at $4^{\circ} \mathrm{C}$. For immunohistochemical processing, free-floating sections were rinsed in TBS after each step of the incubation procedure. Nonspecific background staining was blocked by two preincubation steps: first, $10 \%$ methanol and 7\% hydrogen peroxide in TBS (30 min), and then $1.5 \%$ lysine, $0.25 \%$ Triton X-100 and 10\% bovine serum albumin (BSA) in TBS (60 min) (all from Sigma-Aldrich; St. Louis, $\mathrm{MO}$ ). Sections were then incubated with mouse monoclonal anti-collagen IV primary antibody (C1926, clone COL-94; Sigma) at $4^{\circ} \mathrm{C}$ for 48 h. The antibody was diluted 1:500 in TBS containing $2 \%$ BSA. Subsequently, sections were incubated with secondary antibody (biotinylated anti-mouse IgG; Jackson Im- munoResearch, West Grove, PA; secondary antibody diluted 1:200 in TBS containing 0.2\% donkey serum; Sigma) for $2 \mathrm{~h}$ at room temperature, and then with avidin-biotin peroxidase complex (ABC Vectastain; 1:25 in TBS; Sigma) for another $2 \mathrm{~h}$. 3,3'-Diam-inobenzidine (DAB; $0.8 \mathrm{mg} / \mathrm{ml}$ with $0.003 \%$ hydrogen peroxide; Sigma) was used as a chromogen. To pre- vent shrinkage in section thickness, sections were not dehydrated but mounted on gelatin-coated slides and coverslipped with $80 \%$ glycerol in TBS.

\section{Stereological analysis}

Analysis of cortical gray matter volume was performed with the Cavalieri principle [9] and point counting $[15,44]$. Briefly, a rectangular grid with uniform side length of $15 \mathrm{~mm}$ printed on a transparency was randomly placed on the surface of the 600- to 700-lm-thick sections, 
and all intersections of the grid and the cortical gray matter were counted (on average 477 intersections per hemisphere). Then, the cortical gray matter volume was calculated from the product of the sum of the counted intersections, the uniform area represented by each intersection $\left(15.15=225 \mathrm{~mm}^{2}\right)$, the interval of selecting sections for staining with gallocyanin (2 or 3 ; see above), and the average actual section thickness after tissue processing. Section thickness was determined with the calibrated fine adjustment knob of an Olympus BH microscope and a Zeiss Planapo objective (.20) as de- scribed [22]. The cortical gray matter volume of cases $\mathrm{C} 11$ and $\mathrm{S} 11$ could not be analyzed because sections from the frontal as well as the occipital poles were missing. Capillary length density was investigated with the "Space balls"

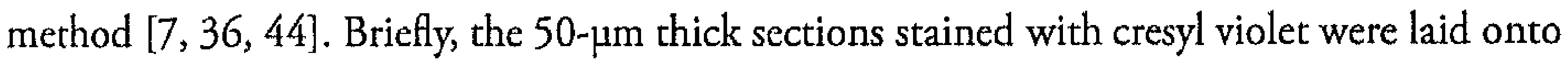
the corresponding adjacent sections processed with immunohistochemistry and were exactly aligned. The regions of interest (areas 9 and 24) were delineated with a stereology workstation, consisting of a modified light microscope (Olympus BX50 with UPlanApo objectives: 10 . for delineation of the regions of interest; 40 , oil, NA=1.0 for Space ball analysis; Olympus, Tokyo, Japan), motorized specimen stage for automatic sampling (Ludl Electronics; Hawthorne, NY), CCD color video camera (HV-C20AMP; Hitachi, Tokyo, Japan) and stereology software (Stereo- Investigator; MicroBrightField, Williston, VT). For this, the sections stained with cresyl violet were brought into focus, these sections were removed, and the sections processed with immunohistochemistry were brought into focus. Hemispheres with radius of 30 $\operatorname{lm}$ (generated by the stereology software while focusing through the section thickness) were systematically and randomly placed within the sections through the regions of interest (on average 365 hemispheres per brain half in area 9, and 426 hemispheres per brain half in area 24) (Fig. 1). The capillary length density was obtained from the total number of intersections between the hemispheres and the capillaries (on average 431 intersections per brain half in area 9, and 448 intersections per brain half in area 24) using the following formula derived from the description of the Space balls method $[9,46]$ according to the sampling scheme applied in the present study, and considering the area of the delineated region of interest and the actual section thickness after tissue processing [55]:

$$
C L D=\frac{2 \times \sum i s\left(D_{X} \times D_{Y} \times t\right)}{2 \times \pi \times r^{3}} \times \frac{1}{V}
$$

where CLD represents the capillary length density, Sis the total number of intersections between the hemi- spheres and the capillaries, DX and DY the distance between the midpoints of the centers of the hemispheres in directions $\mathrm{X}$ and $\mathrm{Y}, \mathrm{t}$ the actual average section thickness after histological processing, $\mathrm{r}$ the radius of the hemispheres, and $\mathrm{V}$ the investigated tissue volume. It is important to note that these capillary length density estimates were based on the analysis of thick sections in three dimensions (3D), and therefore not dependent on the plane of section, the spatial arrangement of the capillaries or their diameter. To focus the analysis on capillaries and to exclude arterioles and venules, the diameter of all vessels coming 
into view during the space ball analysis was measured with the "Fast measure line" tool of the stereology software, and vessels with a diameter greater than $9 \mathrm{~lm}$ were not counted.

Cortical thickness was measured on the 50-1m-thick sections stained with cresyl violet with the "Fast mea- sure line" tool of the stereology software ( 10 objective). To consider variations in cortical thickness caused by oblique sectioning, equidistant traverses [43] spanning layers I-VI with distance between two neighboring traverses of $1 \mathrm{~mm}$ (measured at the top of layer I) were generated, and the mean cortical thickness per brain half was calculated from the mean length of the traverses.

Statistical analysis

For each group (schizophrenics, controls) and hemi- sphere (left, right), mean and standard error of the mean (SEM) were calculated for all investigated parameters. Comparisons between groups were performed with generalized linear models (GLM) multivariate analysis, with diagnosis and hemisphere as fixed factors, all investigated parameters (cortical gray matter volume, cortical thickness of both areas 9 and 24, and capillary length density within both areas 9 and 24) as dependent variables, and patients' age, postmortem interval and fixation time as covariates. All dependent variables were investigated simultaneously. An effect was considered statistically significant if its associated $P$ value was smaller than 0.05 . Calculations were performed using SPSS (Version 11.5 for Windows, SPSS, Chicago, IL).

\section{Photography}

Photomicrographs shown in Fig. 1a-d were produced by digital photography using the stereology workstation described above. On average approximately 125 images were captured for the composite in each Fig. $1 \mathrm{a}-\mathrm{d}$ to represent the entire cortical thickness of area 9 (or area 24, respectively). These images were made into one montage using the Virtual Slice module of the Stereo- Investigator Software (MicroBrightField). Photomicrographs shown in Fig. le - $\mathrm{h}$ were produced by digital photography using an Olympus U-CMAD-2 digital camera attached to an Olympus AX 70 microscope and image analysis software (analySIS; Imaging System, Munster, Germany). The final figures were constructed using Corel Photo-Paint v.11 and Corel Draw v.11 (Corel, Ottawa, Canada). Adjustments of contrast and brightness were made to facilitate recognition of the immunohistochemical signal at low and high magnification, without altering the appearance of the original materials.

\section{Results}

In a pilot study, the cortical gray matter volume of the investigated brains was analyzed and found to be on average significantly reduced in brains from schizophrenics compared to controls ( $P=0.045 ; 9.4 \%$ overall difference between schizophrenics and controls; $10.7 \%$ difference on the left side and $8.0 \%$ difference on the right) (Fig. 2; Table 2 provides an 
overview on all P values of the multivariate analysis). Cortical thickness (layers I-VI) was found to be on average significantly reduced in area 24 in schizophrenics $(\mathrm{P}<0.001 ; 10.6 \%$ overall difference between schizophrenics and controls; $11.7 \%$ difference on the left side and $10 \%$ on the right) but not in area $9(\mathrm{P}=0.368)$ (Fig. 2). Microscopic inspection of immunohistochemically stained sections showed no overt differences in the pattern and the density of capillaries between schizophrenics and controls (Fig. 1). Stereological analysis revealed that the mean values of all samples from schizophrenics (area 9:435.9 $\pm 8.8 \mathrm{~mm} /$ $\mathrm{mm}^{3}$; area $24: 391.7 \pm 10.5 \mathrm{~mm} / \mathrm{mm}^{3}$; mean $\pm \mathrm{SEM}$ ) did not differ from the corresponding mean values of all samples from controls (area $9: 434.5 \pm 12.7 \mathrm{~mm} / \mathrm{mm}^{3} ; 0.3 \%$ overall difference between schizophrenics and controls; area $24: 380.3 \pm 6.5 \mathrm{~mm} / \mathrm{mm}^{3} ; 3.0 \%$ overall difference between schizophrenics and controls) (Fig. 2). Further analysis using each hemisphere separately indicated that diagnosis had no significant effect on the mean capillary length density within area $9(\mathrm{P}=0.498)$ and area $24(\mathrm{P}=0.056)$; only the combination of diagnosis and hemisphere had a significant effect on the mean capillary length density within both areas 9 and $24(\mathrm{P}=0.038$ and $\mathrm{P}=0.033$, respectively) (Fig. 2). However, this did not indicate an overall reduction (or increase, respectively) in mean capillary density within the frontal cortex of the schizophrenics compared to the controls. Rather, the schizophrenics differed slightly compared to the controls in the asymmetry of the mean capillary length density between the left and the right hemisphere in both areas 9 and 24 (Fig. 2). Both schizophrenics and controls had a larger mean capillary length density in the right hemisphere compared to the left, which was found to be significant in area 24 ( $\mathrm{P}=0.002 ; 15.6 \%$ difference in schizophrenics and $4.6 \%$ difference in controls) but not in area $9(\mathrm{P}=0.117 ; 2.8 \%$ difference in schizophrenics and $13.6 \%$ difference in controls). The patients' age, postmortem interval, and fixation time had no signifi- cant effect on any of the investigated parameters.

\section{Discussion}

This is the first rigorous analysis of capillary length density in the frontal cortex on a wellcharacterized sample of postmortem brains from schizophrenics in comparison to controls. The finding of a significantly reduced mean cortical gray matter volume in brains from schizophrenics compared to controls indicated that the sample of brains from schizophrenics investigated in this study shares a cardinal neuropathological feature of schizophrenia with previously reported case studies [17]. The slightly reduced mean cortical thickness in area 24 but not in area 9 in brains from schizophrenics was also in line with previous reports $[4,47,56]$. Thus, the schizophrenic brains investigated in this study showed typical features of the neuropathology of schizophrenia, but no significant alterations in the mean capillary length density within the frontal cortex compared to brains from controls.

As the biological reason for the slightly reduced mean cortical thickness in area 24 in 
brains from schizophrenics is unknown, one can only speculate why no significant alteration in the mean capillary length density was found within this area. At first glance one could hypothesize that the $10.6 \%$ overall reduction in the mean cortical thickness of area 24 and the $3.0 \%$ overall increase in the mean capillary length density within this area in brains from schizophrenics compared to controls was due to compression of the brains from schizophrenics during tissue processing. However, the proto- cols used in the present study did not introduce selective compression of area 24 . Furthermore, a reduced mean cortical thickness in area 24 in brains from schizophrenics was also reported in another study [4]. More- over, on the left side both mean cortical thickness of area 24 and mean capillary length density within this area were reduced in brains from schizophrenics (albeit the latter was not significant), whereas, on the right side, the mean cortical thickness of area 24 was reduced and the mean capillary length density within this area was reduced in brains from schizophrenics. As the same trends were found for area 24 in brains from controls, one could hypothesize a slight compression of area 24 in the right hemisphere in both schizophrenics and controls during neurodevelopment, occurring after formation of the capillary bed, perhaps in connection with the slightly increased mean cortical gray matter volume of the right hemisphere found for both schizophrenics and controls $(4.1 \%$ difference in schizophrenics and $1.3 \%$ difference in controls). However, this will need further investigation of human brain development.

Our data do not support the hypothesis that micro- vasculature abnormalities are a key alteration in the frontal cortex in schizophrenia [39]. Therefore, changes in capillary length density in the frontal cortex cannot be considered a general feature of schizophrenia, and it is unlikely that some type of chronic hypoxia-ischemia occurs in the frontal cortex due to structural microvascular abnormalities. Vice versa, chronic exposure of the frontal cortex to hypoxiaischemia during fetal development or later in life, usually resulting in structural microvascular abnormalities [30], is neither a general event in the neuropathology of schizophrenia.

In two recent studies, reductions in resting regional cerebral blood flow $(\mathrm{rCBF})$ within the frontal cortex of schizophrenics were reported [33, 45]. These reports were in contrast to earlier findings of an increased resting rCBF within the frontal cortex of schizophrenics [8] without providing an explanation for this discrepancy. Impor- tantly, the results of these studies are not necessarily contradictory to the findings of the present study. Rather, recent work on transgenic mice expressing human vas- cular endothelial growth factor (VEGF) has shown that the capillary length density within the cortex does not predict resting $\mathrm{rCBF}$ within the cortex [52]. Despite a significantly increased capillary length density within the frontal cortex (approximately 20\%) and only 3\% of the capillaries not being perfused, the VEGF transgenic mice showed on average less than 10\% increase in resting $\mathrm{rCBF}$ within the cortex (not significant) [52]. Interestingly, during severe hypercapnia, which was accompanied by maximal vasodilation, the VEGF transgenic mice even showed a slight reduction in $\mathrm{rCBF}$ within the cortex compared to non-transgenic controls. These findings point to functional changes in the vasculature itself or in neuronal and glial metabolism, which can be affected in neuropsychiatric disorders [27]. 
In summary, the results of the present study show that alterations in the capillary length density cannot account for the microvasculature abnormalities in the frontal cortex in schizophrenia hypothesized by Prabakaran et al. [39] as the reason for mitochondrial dysfunction, compromised brain metabolism, and oxidative stress in the frontal cortex of schizophrenics. Rather functional changes in the vasculature itself in schizophrenia or in neuronal and glial metabolism should be addressed in further studies. In this regard it should be noted that collagen IV is present only in the basal membranes of microvessels. Thus, it remains possible that endothelial cells themselves have subcle abnormalities. Pericytes surrounding microvessels and vascular smooth muscle cells of larger arterioles will require further investigation, as these cells are important for the regulation of $\mathrm{CBF}$.

Acknowledgements The authors thank G. Greene and B. Boehringer (MicroBrightField, Williston, VT) for valuable help in implementing the "Space balls" method. This study was supported by the Stanley Medical Research Institute (grant no. 02R-258 to H.H., P.R.H. and C.S.), by the European Community (Quality of Life and Management of Living Resources, QLK6-CT-2000-60042, QLK6-GH-00-60042-46, to P.K.), and NIH grant MH66392 (to P.R.H.). R.S.K. was an investigator at the Cerebral Vascular Disease Research Center (NIH grant NS05820). P.R.H. is the Regenstreif Professor of Neuroscience.

\section{References}

1. Andreasen NC, Rezai K, Alliger R, Swayze VW, Flaum M, Kirchner P, et al (1992) Hypofrontality in neurolepric-naive patients and in patients with chronic schizophrenia. Assess- ment with xenon 133 singlephoton emission computed tomography and the Tower of London. Arch Gen Psychiatry 49:943-958

2. Arnold SE, Trojanowski JQ (1996) Recent advances in defining the neuropathology of schizophrenia. Acta Neuropathol 92:217-231

3. Berman KF, Zec RF, Weinberger DR (1986) Physiologic dysfunction of dorsolateral prefrontal cortex in schizophrenia. II. Role of neuroleptic treatment, attention, and mental effort. Arch Gen Psychiatry 43:126-135

4. Bouras C, Kovari E, Hof PR, Riederer BM, Giannakopoulos P(2001) Anterior cingulate cortex pathology in schizophrenia and bipolar disorder. Acta Neuropathol 102:373-379

5. Braak H, Braak E (1995) Staging of Alzheimer's disease-related neurofibrillary changes. Neurobiol Aging 16:271-278

6. Brodmann K (1909) Vergleichende Lokalisationslehre der Großhirnrinde. Barth, Leipzig

7. Calhoun ME, Mouton PR (2000) Length measurement: new developments in neurostereology and 3D imagery. J Chem Neuroanat 21:257-265

8. Catafau AM, Parellada E, Lomena FJ, Bernardo M, Pavia J, Ros D, et al (1994) Prefrontal and temporal blood flow in schizophrenia: resting and activation technetium-99m-HMPAO-SPECT patterns in young neuroleptic-naive patients with acute disease. J Nucl Med 35:935-941

9. Cavalieri B (1635) Geometria indivisibilibus continuorum. Typis Clementis Ferronij, Bononiae (reprinted 1966 as Geometria Degli Indivisibili. Unione Tipografico-Editrice Torinese, Torino) 
10. Cohen BM, Yurgelun-Todd D, English CD, Renshaw PF (1995) Abnormalities of regional distribution of cerebral vasculature in schizophrenia detected by dynamic susceptibility contrast MRI. Am J Psychiatry 152:1801-1803

11. Curtis CE, Iacono WG, Beiser M (1999) Relationship between nailfold plexus visibility and clinical, neuropsychological, and brain structural measures in schizophrenia. Biol Psychiatry 46:102-109

12. Davis KL, Stewart DG, Friedman JI, Buchsbaum M, Harvey PD, Hof PR, Buxbaum J, Haroutunian V (2003) White matter changes in schizophrenia: evidence for myelin-related dys- function. Arch Gen Psychiatry 60:443-456

13. Falkai P, Honer WG, David S, Bogerts B, Majtenyi C, Bayer TA (1999) No evidence for astrogliosis in brains of schizo- phrenic patients. A post-mortem study. Neuropathol Appl Neurobiol 25:48-53

14. Gittins R, Harrison PJ (2004) A quantitative morphometric study of the human anterior cingulate cortex. Brain Res 1013:212-222

15. Gundersen HJG, Jensen EB (1987) The efficiency of systematic sampling and its prediction. J Microsc 147:229-263

16. Hakak Y, Walker JR, Li C, Wong WH, Davis KL, Buxbaum JD, Haroutunian V, Fienberg AA (2001) Genome-wide expression analysis reveals dysregulation of myelination-related genes in chronic schizophrenia. Proc Natl Acad Sci USA 98:4746-4751

17. Harrison PJ (1999) The neuropathology of schizophrenia. A critical review of the data and their interpretation. Brain 122:593-624

18. Harrison PJ, Owen MJ (2003) Genes for schizophrenia? Recent findings and their pathophysiological implications. Lancet 361:417-419

19. Harrison PJ, Weinberger DR (2004) Schizophrenia genes, gene expression, and neuropathology: on the matter of their convergence. Mol Psychiatry: advance online publication, 20 July 2004, doi:10.1038/ sj.mp. 4001558

20. Heinsen H, Heinsen YL (1991) Serial thick, frozen, gallocyanin stained sections of human central nervous system. J Histotechnol 14:167-173

21. Heinsen H, Beckmann H, Heinsen YL, Gallyas F, Haas S, Scharff G (1989) Laminar neuropathology in Alzheimer's disease by a modified Gallyas impregnation. Psychiatry Res 29:463-465

22. Heinsen H, Henn R, Eisenmenger W, Gotz M, Bohl J, Bethke B, et al (1994) Quantitative investigations on the human entorhinal area: left-right asymmetry and age-related changes. Anat Embryol (Berl) 190:181-194

23. Heinsen H, Arzberger T, Schmitz C (2000) Celloidin mounting (embedding without infiltration) - a new, simple and reliable method for producing serial sections of high thickness through complete human brains and its application to stereological and immunohistochemical investigations. J Chem Neuroanat 20:49-59

24. Hill K, Mann L, Laws KR, Stephenson CM, Nimmo-Smith I, McKenna PJ (2004) Hypofrontality in schizophrenia: a metaanalysis of functional imaging studies. Acta Psychiatr Scand 110:243-256

25. Hof PR, Haroutunian V, Copland C, Davis KL, Buxbaum JD (2002) Molecular and cellular evidence for an oligodendrocyte abnormality in schizophrenia. Neurochem Res 27:1193-1200

26. Hof PR, Haroutunian V, Friedrich VL, Jr, Byne W, Buitron C, Perl DP, Davis KL (2003) Loss and altered spatial distribution of oligodendrocytes in the superior frontal gyrus in schizophrenia. Biol Psychiatry 53:1075-1085 
27. ladecola $C$ (2004) Neurovascular regulation in the normal brain and in Alzheimer's disease. Nat Rev Neurosci 5:347-360

28. Kety SS (1959) Biochemical theories of schizophrenia. I. Science 129:1528-1532

29. Kety SS (1959) Biochemical theories of schizophrenia. II. Science 129:1590-1596

30. LaManna JC, Chavez JC, Pichiule P (2004) Structural and functional adaptation to hypoxia in the rat brain. J Exp Biol 207:3163-3169

31. Lewis DA, Lewitt P (2002) Schizophrenia as a disorder of neurodevelopment. Annu Rev Neurosci 25:409432

32. Lieberman JA (1999) Is schizophrenia a neurodegenerative disorder? A clinical and neurobiological perspective. Biol Psychiatry 46:729-739

33. Malaspina D, Harkavy-Friedman J, Corcoran C, Mujica-Parodi L, Prinzz D, Gorman JM, Van Heertum R (2004) Resting neural activity distinguishes subgroups of schizophrenia patients. Biol Psychiatry 56:931-937

34. Meyer-Lindenberg A, Miletich RS, Kohn PD, Esposito G, Carson RE, Quarantelli M, Weinberger DR, Berman KF (2002) Reduced prefrontal activity predicts exaggerated striatal dopaminergic function in schizophrenia. Nat Neurosci 5:267-271

35. Middleton FA, Mirnics K, Pierri JN, Lewis DA, Levitt P (2002) Gene expression profiling reveals alterations of specific metabolic pathways in schizophrenia. J Neurosci 22:2718-2729

36. Mouton PR, Gokhale AM, Ward NL, West MJ (2002) Stereological length estimation using spherical probes. J Microsc 206:54-64

37. Mueser KT, McGurk SR (2004) Schizophrenia. Lancet 363:2063-2072

38. Nimchinsky EA, Vogt BA, Morrison JH, Hof PR (1997) Neuroflament and calcium-binding proteins in the human cingulate cortex. J Comp Neurol 384:597-620

39. Prabakaran S, Swatton JE, Ryan mm, Huffaker SJ, Huang JT, Griffin JL, et al (2004) Mitochondrial dysfunction in schizophrenia: evidence for compromised brain metabolism and oxidative stress. Mol Psychiatry 9:684-697

40. Rajkowska G, Goldman-Rakic PS (1995) Cytoarchitectonic definition of prefrontal areas in the normal human cortex. I. Remapping of areas 9 and 46 using quantitative criteria. Cereb Cortex 5:307-322

41. Rajkowska G, Goldman-Rakic PS (1995) Cytoarchitectonic definition of prefrontal areas in the normal human cortex. II. Variability in locations of areas 9 and 46 and relationship to the Talairach Coordinate System. Cereb Cortex 5:323-337

42. Ross BM, Hughes B, Turenne S, Seeman M, Warsh JJ (2004) Reduced vasodilatory response to methylnicotinate in schizophrenia as assessed by laser Doppler flowmetry. Eur Neuro-psychopharmacol 14:191-197

43. Schleicher A, Amunts K, Geyer S, Kowalski T, Schormann T, Palomero-Gallagher N, Zilles K (2000) A stereological approach to human cortical architecture: identification and delineation of cortical areas. J Chem Neuroanat 20:31-47

44. Schmitz C, Hof PR (2005) Design-based stereology in neuroscience. Neuroscience 130:813-831

45. Schultz SK, O'Leary DS, Boles Ponto LL, Arndt S, Magnotta V, Watkins GL, et al (2002) Age and regional cerebral blood flow in schizophrenia: age effects in anterior cingulate, frontal, and parietal cortex. J Neuropsychiatry Clin Neurosci 14:19-24

46. Selemon LD, Goldman-Rakic PS (1999) The reduced neuropil hypothesis: a circuit based model of schizophrenia. Biol Psychiatry 45:17-25 
47. Selemon LD, Rajkowska G, Goldman-Rakic PS (1995) Abnormally high neuronal density in the schizophrenic cortex. A morphometric analysis of prefrontal area 9 and occipital area 17. Arch Gen Psychiatry $52: 805-818$

48. Siever LJ, Davis KL (2004) The pathophysiology of schizophrenia disorders: perspectives from the spectrum. Am J Psychiatry 161:398-413

49. Sofroniew MV (2000) Astrocyte failure as a cause of CNS dysfunction. Mol Psychiatry 5:230-232

50. Tkachev D, Mimmack ML, Ryan mm, Wayland M, Freeman T, Jones PB, Starkey M, Webster MJ, Yolken RH, Bahn S (2003) Oligodendrocyte dysfunction in schizophrenia and bipolar disorder. Lancet 362:798805

51. Tsuang M (2000) Schizophrenia: genes and environment. Biol Psychiatry 47:210-220

52. Vogel J, Gehrig M, Kuschinsky W, Marti HH (2004) Massive inborn angiogenesis in the brain scarcely raises cerebral blood flow. J Cereb Blood Flow Merab 24:849-859

53. Vogt BA, Nimchinsky EA, Vogt LJ, Hof PR (1995) Human cingulate cortex: surface features, flat maps, and cytoarchitecture. J Comp Neurol 359:490-506

54. Von Economo C, Koskinas GN (1925) Die Cytoarchitekonik der Hirnrinde des erwachsenen Menschen. Julius Springer, Wien, Berlin

55. West MJ, Slomianka L (1998) Total number of neurons in the layers of the human entorhinal cortex (Erratum). Hippocampus 8:426

56. Wiegand LC, Warfield SK, Levitt JJ, Hirayasu Y, Salisbury DF, Heckers S (2004) Prefrontal cortical thickness in first-episode psychosis: a magnetic resonance imaging study. Biol Psychiatry 55:131-140 


\section{Tables}

Table 1. Clinical characteristics of all cases included in this study

\begin{tabular}{|c|c|c|c|c|c|c|c|}
\hline No & A & 0 & Cause of death & PMI & Fix & \multicolumn{2}{|c|}{ Diagnosis } \\
\hline & (y) & (y) & & (h) & (d) & DSM-IV & ICD-10 \\
\hline$\$ 1$ & 22 & 19 & Suicidea & 88 & 130 & 295.30 & $F 20.00$ \\
\hline$\$ 2$ & 36 & 28 & Suicidea & $<72$ & 115 & 295.30 & F20.00 \\
\hline 53 & 46 & 24 & Systemic hypothermia & $<24$ & 327 & 295.30 & $F 20.01$ \\
\hline 54 & 50 & 17 & Peritonitis & $<24$ & 203 & 295.30 & $F 20.00$ \\
\hline 55 & 50 & 22 & Suicide & 18 & 170 & 295.30 & $F 20.00$ \\
\hline 56 & 51 & 17 & Septicaemia & 33 & 127 & 295.60 & F20.50 \\
\hline 57 & 54 & 20 & Septicaemia & 27 & 250 & 295.60 & F20.50 \\
\hline 58 & 55 & 22 & Right-sided heart failure & 25 & 84 & 295.30 & $F 20.00$ \\
\hline 59 & 57 & 37 & Septicaemia & 76 & 163 & 295.30 & F20.00 \\
\hline 510 & 60 & 24 & Pulmonary embolism & $<48$ & 311 & 295.30 & $F 20.01$ \\
\hline$S 11^{b}$ & 62 & 19 & Aspiration & 7 & 171 & 295.30 & $F 20.00$ \\
\hline 512 & 63 & 22 & Acute myocardial infarct & 15 & 338 & 295.60 & F20.50 \\
\hline$\$ 13$ & 64 & 26 & Pulmonary embolism & 6 & 817 & 295.60 & F20.50 \\
\hline $\mathrm{C} 1$ & 25 & - & Cardiac tamponade & 14 & 119 & - & - \\
\hline$C 2$ & 36 & - & Gunshot & 24 & 143 & - & - \\
\hline C3 & 47 & - & Acute myocardial infarct & $<24$ & 133 & - & - \\
\hline $\mathrm{C4}$ & 50 & - & Acute myocardial infarct & 35 & 433 & - & - \\
\hline $\mathrm{C} 5$ & 50 & - & Avalanche accident & 23 & 498 & - & - \\
\hline C6 & 51 & - & Septicaemia & 7 & 285 & - & - \\
\hline$C 7$ & 54 & - & Acute myocardial infarct & 18 & 168 & - & - \\
\hline $\mathrm{C} 8$ & 56 & - & Acute myocardial infarct & 60 & 3570 & _- & - \\
\hline C9 & 58 & - & Acute myocardial infarct & 28 & 126 & - & - \\
\hline $\mathrm{C} 10$ & 60 & - & Gastrointestinal haemorrhage & 18 & 101 & - & - \\
\hline $\mathrm{C} 11^{\mathrm{b}}$ & 60 & - & Gastrointestinal haemorrhage & 27 & 302 & - & - \\
\hline $\mathrm{C} 12$ & 62 & - & Acute myocardial infarct & $<24$ & 3696 & - & - \\
\hline$C 13$ & 65 & - & Bronchopneumonia & 6 & 2289 & - & - \\
\hline
\end{tabular}

$\mathrm{S}=$ schizophrenic patient; $\mathrm{C}=$ control; $\mathrm{A}=$ age at death; $\mathrm{O}=$ age at onset; $\mathrm{PMI}=$ post-rnortem interval (time between death and autopsy); $F i x=$ fixation time; $y=$ years; $h=$ hours; $d=$ days. aThese two patients had relatively long post-mortem intervals. However, both had committed suicide (one by hanging, the other by jumping from a building), were found within $1 \mathrm{~h}$ of death and were kept at $4^{\circ} \mathrm{C}$ until autopsy. Accordingly, the post-mortem intervals between death and autopsy of these patients cannot be compared with the corresponding intervals of the other cases and were thus excluded from the calculation of the mean post-mortem intervals. "TThe volume of the CGM of these cases could not be analysed because sections from the frontal as well as the occipital poles were missing. 
Table 2. Results of generalized linear models (GLM) multivariate analysis ( $p$ values). $P$ values smaller than 0.05 are shown in boldface (CGMV cortical gray matter volume, $T$ cortical thickness, CLD capillary length density, $\mathrm{D}$ diagnosis, $\mathrm{H}$ hemisphere)

\begin{tabular}{cccccc} 
Source & CGMV & 9-T & 9-CLD & 24-T & 24-CLD \\
\hline Age & 0.852 & 0.680 & 0.821 & 0.164 & 0.193 \\
PMI & 0.844 & 0.473 & 0.804 & 0.189 & 0.078 \\
Fix & 0.812 & 0.731 & 0.217 & 0.090 & 0.971 \\
D & 0.045 & 0.368 & 0.498 & $<0.001$ & 0.056 \\
H & 0.730 & 0.417 & 0.117 & 0.838 & 0.002 \\
D.H & 0.881 & 0.644 & 0.038 & 0.454 & 0.033 \\
\hline
\end{tabular}

Figures

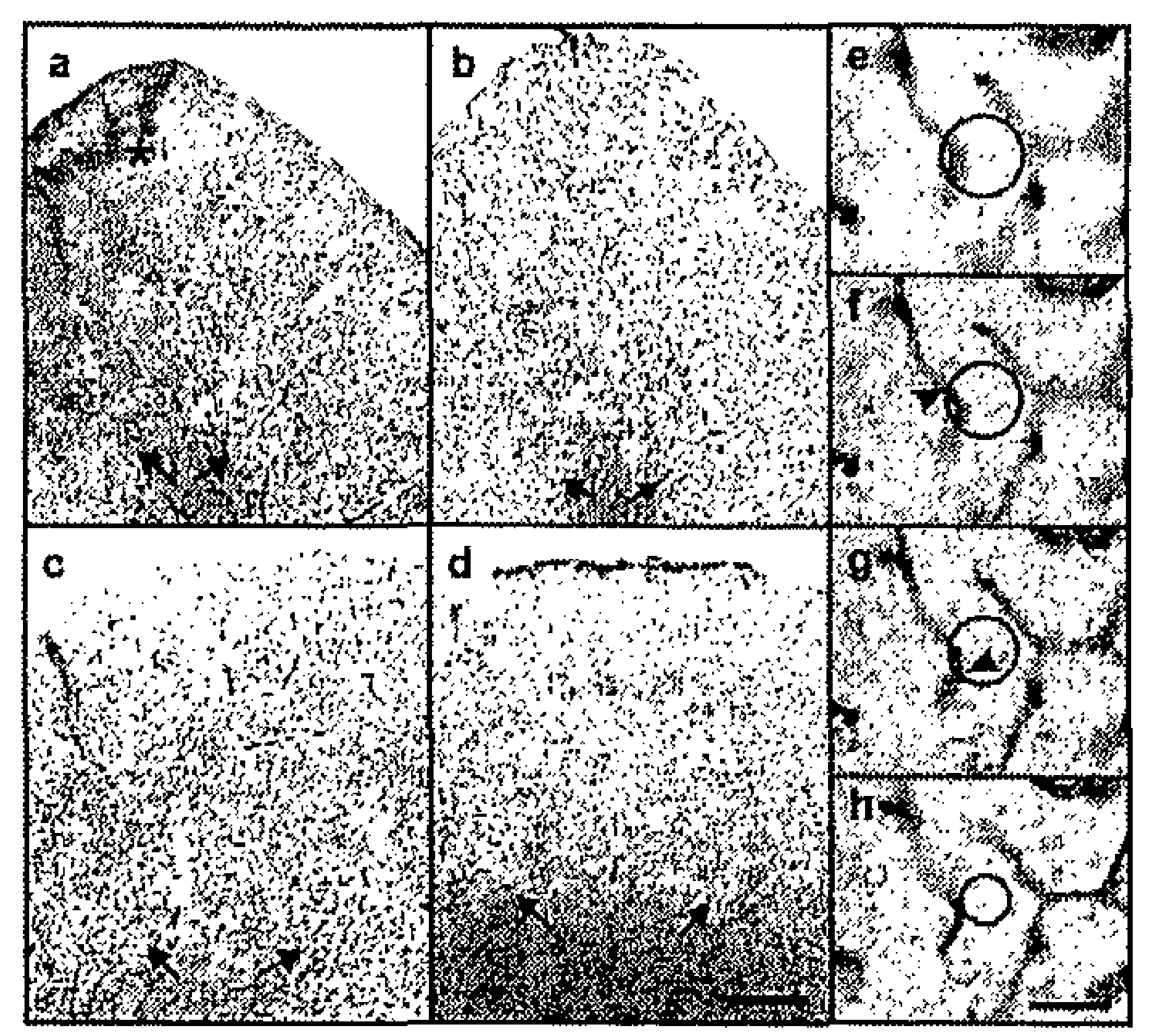

Fig. 1. Analysis of capillary length density with the "Space balls" method. a-d Representative lowpower photomicrographs of 50-1m-thick coronal sections from the left brain half of a schizophrenic patient $(a, c)$ and a control $(b, d)$ showing immunohistochemical detection of collagen IV in area 9 $(a, b)$ and area $24(c, d)$. Arrows indicate the border between layer $V l$ andthe white matter. The asterisk points to an artifact, which, at high magnification, did not disturb the analysis. e-h Representativehigh-power photomicrographs showing the same microscopic field at four consecutive focal planes below the upper surface, with a distance of $8 \mathrm{Im}$ between the focal planes. The intersections of a hemisphere (a "space ball") with a radius of $30 \mathrm{Im}$ and the focal plane at the investigated focal depth are shown as circles. Intersections between the hemisphere and the capillaries in focus at the point of intersection are marked with arrowheads. Bars a-d $1 \mathrm{~mm}, \mathrm{e}-\mathrm{h} 60 \mu \mathrm{m}$ 

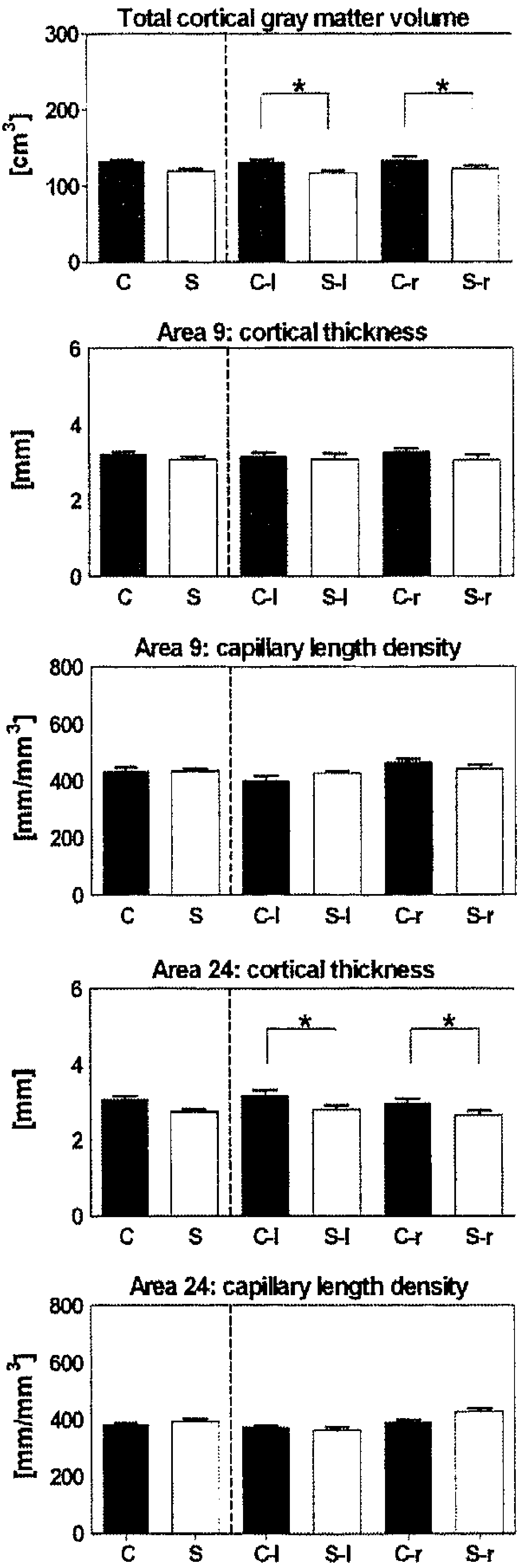

Fig. 2. Results of the stereological analysis of the total cortical gray matter volume as well of the cortical thickness of areas 9 and 24 and the capillary length density within these areas from 13 male schizophrenics (open bars) and from 13 age-matched male controls (closed bars). On the left side, all data from schizophrenics and controls are shown (left and right hemispheres); on the right side data are shown separately for the left and the right hemispheres. *Significant difference between schizophrenics and controls with uniform alterations on both sides 


\section{CMTCR}

\section{Neuronal distribution in the neocortex of schizophrenic patients}

Manuel F. Casanovaa, Pawel Kreczmanskib,c, Juan Trippe Ila, Andrew Switalaa Helmut Heinsen ${ }^{d}$, Harry W.M. Steinbusch ${ }^{\mathrm{b}, c}$, and Christoph Schmitz ${ }^{\mathrm{b}, c}$

aDepartment of Psychiatry and Behavioral Sciences, University of Louisville, Louisville, KY 40292, USA

'Department of Psychiatry and Neuropsychology, Division of Cellular Neuroscience, Maastricht University, Maastricht, Netherlands

'European Graduate School of Neuroscience (EURON), Maastricht, Netherlands "Morphological Brain Research Unit, University of Wuerzburg, Wuerzburg, Germany 


\begin{abstract}
It has been postulated that the prefrontal cortices of schizophrenic patients have significant alterations in their neuropil space. However, previous results have been contradictory and inconclusive, reporting both decreases and increases in the prefrontal neuropil. The present study re-examines these findings based on measurements of cell density, and intercellular distances within and between cell minicolumns. The results indicate alterations in the neuropil of schizophrenic patients according to both the lamina and cortical area examined. Alterations were present in all cortical areas studied. The findings suggest an alteration in the modulatory systems innervating the cell minicolumn. Furthermore, the lack of variation in core columnarity parameters argues in favor of a defect postdating the formation of the cell minicolumn.
\end{abstract}




\section{Introduction}

The minicolumn is a basic architectonic motif of mammalian neocortex, consisting of a core of pyramidal neurons and their associated apical dendritic and myelinated bundles oriented perpendicularly to the pial surface (Mountcastle, 1997; Buxhoeveden and Casanova, 2002). Around the circumference of this core is a cell-sparse peripheral neuropil space containing inhibitory interneurons, glia, basilar dendritic and collateral axonal elements, capillaries and synaptic elements. Changes in minicolumnar morphometry have been associated with neuropsychiatric pathology such as autism (Casanova et al., 2006a). A recent study analyzing mean cell spacing (MCS) in minicolumns of schizophrenic patients indicated significant interactions by both lamina and cortical area examined (Casanova et al., 2005). Parameters in this study also modeled pyramidal cell core integrity with indices of columnar width (CW) and a verticality index (VI) (representing relative distribution of somas along the radial axis). The study found no significant changes in these minicolumnar parameters.

The structure of each neocortical layer reflects a characteristic organization of dendritic arbors, synaptic components, and other elements. Mesocortical and basal forebrain projections of modulatory neurotransmitters ramify tangentially throughout the neocortex. These projections act via lamina-specific distributions of monoamine receptors predominately within neuropil (Benavides-Piccione et al., 2005) the largest compartment of which is the peripheral neuropil space surrounding the pyramidal cell core. Significant MCS changes in the context of stable values for both VI and CW in schizophrenia are consistent with an abnormality of cortical lamination. The implication is that the source(s) of pathology originates outside of the cortex and is independent of the core components of the minicolumn (Casanova et al., 2005).

The minicolumnar pyramidal cell core develops from an ontogenetic cell column in the fetal cortical plate comprising clonally-related neuroblasts migrating radially outward along a common radial glial fiber (Rakic, 1988, 1995). Its morphometry, including column width and radial orientation, is constrained by tangential distribution of progenitors arising from symmetric divisions in the germinal neuroepithelium and asymmetric divisions yielding the full complement of postmitotic daughter neurons within each ontogenetic column and is conserved over the course of prenatal and postnatal development (Casanova et al., 2006b). Lamination arises subsequent to formation of this columnar core cytoarchitecture (Kostović and Rakic, 1990) and concurrent with elaboration of mesocortical monoaminergic inputs ramifying tangentially through the cortical plate (Lambe et al., 2000; Verney, 2003). The largest proportion of local monoaminergic appositions is located in neuropil on dendritic processes (Benavides-Piccione et al., 2005). Monoaminergic inputs vary with regional and laminar cytoarchitecture (Berger et al., 1988), and alterations of monoaminergic input are associated with changes in dendritic structure and neuropil composition in schizophrenics (Benes et al., 2000). Lamina-specific development of neuropil elements, including migration of interneurons and glia, growth of dendritic arborizations and super- 
ficial axon collaterals affects neuronal spacing both within and between minicolumn cores. Changes in MCS in schizophrenic brains thus may reflect lamina-specific neuropil changes resulting from altered monoaminergic inputs during development.

In the present study we used a Boolean model (Hahn et al., 1999) to calculate the number of cells and the size of the soma from the same tissue slides as previously reported (Casanova et al., 2005). In this method, computer assisted image processing of coronal brain tissue photomicrographic sections generate characteristic radially-oriented periodic texture gradients corresponding to minicolumnar periodicity. Cell density is represented by the parameter $\lambda$, which incorporates values for particle surface area and intensity. This allows for comparisons of particle density across core and peripheral neuropil. We also obtained gray-level index values (GLI) corresponding to the relative proportion of somatic staining according to a modified method of Schleicher and colleagues (2005). A Delaunay triangulation method was employed to determine the distribution of intracolumnar relative to intercolumnar edge lengths between particles. Changes in the bimodal distribution for these values reflect lamina- and area-specific changes in density and organization of neuropil versus the minicolumn's pyramidal cell core.

\section{Methods}

\section{Brain specimens}

We investigated post mortem brains (both hemispheres) from 13 male schizophrenics with a mean age of 51.5 years (standard deviation [s.d.] 11.8 years) and a mean interval between death and autopsy of 27.6 hours (s.d. 19.9 hours). Controls consisted of 13 age-matched males with a mean age of 51.9 years (s.d. 11.1 years) and a mean interval between death and autopsy of 23.7 hours (s.d. 13.6 hours). Clinical diagnoses, causes of death and the interval between death and autopsy are summarized in Table 1. All schizophrenics were long-term patients at German university hospitals or at German state psychiatric hospitals. Complete clinical records were available in all cases. Records from autopsy (including a summary of the medical history) were available for all controls. All of the patients met the Diagnostic Statistical Manual, 4th revision (DSM-IV) and International Statistical Classification of Diseases and Related Health Problems, 10th revision (ICD-10) diagnostic criteria for schizophrenia. The clinical notes were assessed by experienced clinical psychiarrists (mentioned in Acknowledgements) to ensure that the control brains were free from psychopathology, and for clear evidence that the schizophrenic cases conformed to DSM-IV criteria for schizophrenia. In particular, the schizophrenic group included no patient diagnosed with schizoaffective disorder. Exclusion criteria comprised neurological problems that required intervention or interfered with cognitive assessment (e.g., stroke with aphasia), history of recurrent seizure disorder (i.e., epilepsy), history of severe head injury with loss of consciousness, diabetes mellitus with poor glycemic control (free plasma 
glucose $>200 \mathrm{mg} / \mathrm{dl}$ ), and history of self-induced water intoxication with hyponatremia. Brains from schizophrenics were also excluded if patients had undergone a leukotomy. The two groups - schizophrenics and controls - were similar in terms of the ethnic backgrounds of the subjects. However, they were not fully matched for socio-economical status and education, which would have placed severe constraints on our sample. Moreover, all schizophrenics were subjected to long-term treatment with high doses of neuroleptics.

In all of the cases, autopsy was performed after consent was obtained from a patient's rclative according to the laws of the Federal Republic of Germany. The use of these autopsy cases for scientific investigations as outlined here has been approved by the relevant Institutional Review Boards (IRB). Brains were fixed by immersion in 10\% formalin (1 part commercial $40 \%$ aqueous formaldehyde in 9 parts $\mathrm{H} 2 \mathrm{O}$ ) for at least 3 months and up to 11 years. As the distortion due to fixation stabilizes after no more than approximately 3 weeks (Quester and Schroeder, 1997), all brains would have reached their stable, post-fixation state when studied. Thus, the duration of fixation can be ruled out as a potential confound.

\section{Tissue processing}

The brainstem with the cerebellum was severed at the level of the rostral pons, and the hemispheres divided medio-sagittally. Then, both hemispheres were cut into serial $600 \mu \mathrm{m}$ to $700 \mu \mathrm{m}$ thick coronal sections as previously described in the literature (Heinsen and Heinsen, 1991). Briefly, the hemispheres were cryoprotected in a mixture of glycerol-dimethylsulfoxide-formalin after carefully removing meninges and pial vesscls. They were then embedded in gelatin, frozen at $-60^{\circ} \mathrm{C}$, and serially sectioned using a cryostat (Jung, Nussloch, Germany). One brain (control case C7) was embedded in celloidin (Heinsen et al., 2000) and cut into serial $440 \mu \mathrm{m}$ thick coronal sections using a sliding microtome (Polycut, Cambridge Instruments, UK). From each hemisphere, every second or third section was stained with gallocyanin (Heinsen and Heinsen, 1991). The remaining sections were stored in formalin for up to ten years.

From all the brains of schizophrenics and controls older than 40 years, sections through the central parts of the entorhinal and transentorhinal cortices were labeled with the Gallyas silver method to detect neurofibrillary changes (Heinsen et al., 1999). Neurofibrillary tangles were rarely detected. In affected patients the findings corresponded to Braak-Braak Alzheimer classification stage I (Braak and Braak, 1995). Furthermore, all of the sections used in this study were coded and controlled for the absence of tumors, infarcts, heterotopias, signs of autolysis, staining artifacts, and gliosis.

Gallocyanin-stained sections were used by two of us (P.K. and C.S.) to identify areas M1, V1, frontal association cortex (areas 4, 17, and 9 of Brodmann [1909], respectively) and S1 (area 3b of Vogt and Vogt [1919]) in both cerebral hemispheres according to anatomical landmarks and cytoarchitectural criteria. Gross anatomical landmarks for M1 include the anterior wall of the central sulcus and adjacent portions of the precentral gyrus. Cytoarchitectonically, the region is clearly demarcated by its giant Betz cells and minimization of layer IV. Area 17 (V1) is located along the walls of the calcarine sulcus in the oc- 
cipital lobe and adjacent portions of the cuneus and lingual gyrus (Carpenter, 1985). It is defined histologically by a broad lamina IV divided into 3 sublayers with numerous very small pyramidal cells in layers II and III. It is noted for the dense line of Gennari in myelin stains. Area 9 lies in the superior and middle frontal gyrus. Rajkowska and Goldman-Rakic (1995a, 1995b) found that it was located in the middle third of the superior frontal gyrus in all the cases they examined. It covered both dorsolateral and dorsomedial surfaces of this gyrus and extended in some cases to the depth of the superior frontal gyrus and portions of the middle frontal gyrus.

Concerning area 9, Rajkowska and associates showed that its more lateral portions embrace area 46 (area FD $\Delta$ of Economo and Koskinas [1925]) (Rajkowska and GoldmanRakic, 1995a, 1995b). It is important to note that Brodmann (1909) never described the cytoarchitectonic characteristics of area 9 in man. Rather he projected his observations in monkey onto the frontal lobe of man. Economo and Koskinas (1925) provided a detailed cytoarchitectonic description of the human frontal lobe. Their area FDm corresponds to area 9. It is characterized by a distinct layer II, absence of very large, slender and wellelaborated pyramidal cells in layer IIIc (typical of area 6 and to a lesser extend of area 8), and an uninterrupted layer IV with fairly definite upper and lower boundaries. In thick gallocyanin-stained sections a cell-poor layer is found between layers IIIc and layer IV. Layer IV is more prominent and cell-rich in the frontopolar regions; however the transition between areas 9 and 10 is a gradual one. Layer $\mathrm{V}$ of area 9 is subdivided into layers $\mathrm{Va}$ and $\mathrm{Vb}$, the latter of which is rather cell-sparse. Most probably the cell-poor layers between lamina IIIc and IV and within layer Vb correspond to the upper and lower band described by Rajkowska and associates in myelin-stained sections of area 9 (Rajkowska and Goldman-Rakic, 1995a, 1995b). Finally, layer VI consists of sublayers VIa and VIb. VIa is cell-rich and its upper border to the cell-sparse layer $\mathrm{Vb}$ can easily be recognized. The lower boundaries of layer $\mathrm{VIb}$ to the underlying white matter are much more distinct than in the caudally ensuing areas 8 and 6. The overall cortical thickness of the frontopolar area 10 (field FE of Economo and Koskinas [1925]) is considerably less than in area 9, lamina Vla is small, rich in darklystained pyramidal as well as spindle-shape cells and the boundary of VIa to the medullary layer is even more distinct than in area 9 . The presence of the small and cell rich layer VI facilitates the delineation of area 9 from the rostral area 10 in thick gallocyanin stained sections. Finally, layer IV is more conspicuous than its corresponding part in area 9.

Regions of interest were removed from adjacent sections stored in formalin and not stained with gallocyanin as $2 \mathrm{~cm} \times 2 \mathrm{~cm}$ tissue pieces. After rinsing in tap water for several hours, the pieces were stored in tris-buffered saline (TBS) overnight $(\mathrm{pH}=9.0)$. They were then placed in TBS ( $\mathrm{pH}=9.0$ ) and heated in a microwave oven for $15 \mathrm{~min}$ at $90^{\circ} \mathrm{C}$ for antigen retrieval (results of immunohistochemical analysis will be published elsewhere). After cooling down, the tissue blocks were rinsed in TBS twice $(\mathrm{pH}=7.4)$ and placed in a TBS based $30 \%$ sucrose solution for cryoprotection. The pieces were then frozen and cut into $50 \mu \mathrm{m}$ thick sections using a cryostat (Frigocut 2800E; Jung, Nussloch, Germany). These $50 \mu \mathrm{m}$ thick sections were stained with cresyl-violet $(0.1 \%, 20 \mathrm{~min})$. 


\section{Computerized image analysis}

Digital images were taken from laminas III and V in cortical areas M1, S1, V1, and Brodmann area 9 in both cerebral hemispheres. Photomicrographs were taken through the flat face of the gyri with minicolumns perpendicular to the pia. The equipment (Nikon E-800 microscope, using a $10 x$ objective and digital camera) was such that each field of view measured approximately $1.45 \mathrm{~mm}^{2}$. Up to three fields were captured per lamina/area/ hemisphere as tissue quality allowed. Of the 416 possible regions of interest (16 in each of 26 brains), 90 were completely lacking in usable tissue; the number of images captured per available region numbered 2.7 .

Color micrographs were converted to intensity (grayscale) images, which were corrected for uneven illumination and subsequently thresholded to produce binary images using using Otsu's (1979) method. Boolean model and GLI parameters (below) were estimated from these bitmaps without further processing. In order to estimate the interneuronal distance distributions, the foreground (dark) regions of the binary images were segmented into connected regions. Clusters of overlapping cell somata, which appear to be single contiguous regions in the binary images, were further separated using the watershed transformation. Connected regions were classified according to size. Objects smaller than 10 $\mu \mathrm{m} 2$, assumed to be glia, neuron fragments, or noise, were discarded altogether. Of the remaining objects, those with areas above the 15 th percentile were taken to be pyramidal cell somata, while the smaller objects were classified as interneurons. The latter threshold was based on the fraction of all neurons estimated to be inhibitory by Braitenberg and Schüz (1998).

Computerized image analysis produced estimates of the surface fraction (i.e. the proportion $A_{A}$ ) of Nissl-stained tissue within the sections, the boundary length $L_{A}$ of stained tissue per unit area, and the planar convexity $N_{A}^{+}$, which quantities suffice to compute parameters of a Boolean model with convex primary grains using the method of moments (Stoyan et al., 1995). In this spatial process, a random closed set (the stained region of an image in our case) is modeled as the union of primary sets (grains) drawn at random from a distribution with mean grain area is $\bar{A}$ and mean grain perimeter is $\bar{U}$. The primary grains, or rather their centroids, are placed at the points of a Poisson process with intensity $\lambda$. These three parameters, which completely determine the Boolean model, were obtained algebraically from the following equations (Stoyan et al., 1995), substituting for $A_{A^{\prime}} L_{A^{\prime}}$ and $N_{A}^{+}$their respective estimates:

$$
\begin{aligned}
& A_{A}=1-e^{-\lambda \bar{A}} \\
& N_{A}^{+}=\lambda\left(1-A_{A}\right) \\
& L_{A}=N_{A}^{+} \bar{U}
\end{aligned}
$$

Interactions between points, such as clumping in minicolumns, are not considered in this simple model. Parameter estimates represent an average across an entire microscopic field. 
Thresholded images were also analyzed according to the gray level index (GLI) method (Schleicher et al., 2005), slightly modified so that the GLI varied smoothly across a region. Here, the inverse thresholded image, with foreground set equal to one and background set equal to zero, was convolved with a kernel measuring $11 \mu \mathrm{m}$ by $110 \mu \mathrm{m}$ at half-maximum. The long axis of the kernel was parallel to the axes of minicolumns, following the image Y-axis. What resulted was an estimate of the GLI, or staining intensity, in the neighborhood of each pixel. Each GLI image so produced was measured in profiles in the $\mathrm{X}$ direction to obtain:

- mean (D) and standard deviation (sdD) in distance between local maxima of the GLI,

- amplitude (A) of the local maxima, or difference between peak GLI and the lowest GLI between the peak in question and the next peak in the profile, and

- mean (W) and standard deviation (sdW) in the width (full width at half maximum) of the peaks.

A follow-up study considered the distance between neighboring objects, excepting those below the $15 \%$ area threshold for pyramidal cells. Objects were considered neighbors if (a) they were endpoints of an edge of the Delaunay triangulation of object centroids, and (b) they were not further apart than the distance from either of them to the edge of the region of interest. If objects in a single lamina are clustered, as cells in minicolumns, an object's neighbors may include members of the same cluster or members of a nearby cluster (Figure 1). The distribution of distances $d$ between neighbors is then a mixture of intracluster or near distances, and intercluster or far distances. This was modeled as a two component lognormal mixture distribution

$$
\log d \sim \alpha N\left(\mu_{\text {near }}, \sigma_{\text {near }}^{2}\right)+(1-\alpha) N\left(\mu_{\text {far }}, \sigma_{\text {far }}^{2}\right) ; 0 \leq \alpha \leq 1
$$

Schizophrenic and normal patients were paired for purposes of statistical analysis. Testing employed multivariate ANOVA with fixed effects diagnosis, cortical area, hemisphere, lamina, and corresponding interactions in a full factorial design. Separate multivariate ANOVA tests were performed for each of the three sets of parameters (Boolean model, GLI, and distance distribution). P values were estimated from Wilks's $\Lambda$, transformed to the equivalent $\mathrm{F}$ statistic.

\section{Results}

Multivariate testing of the Boolean model parameters was significant in the diagnosis by cortical area interaction $(F=3.28$; d.f. $=9,682 ; \mathrm{P}=0.0006)$, the lamina by cortical area interaction $(\mathrm{F}=3.02 ;$ d.f. $=9,682 ; \mathrm{P}=0.0015)$, and the main effects of cortical area $(\mathrm{F}=78.5 ;$ d.f. $=9,682 ; \mathrm{P}<0.0001)$ and lamina $(\mathrm{F}=42.0 ;$ d.f. $=3,682 ; \mathrm{P}<0.0001)$ Of the individual parameters, only $\lambda$ depended significantly on the interaction of diagnosis and cortical area $(F=6.58$; d.f. $=3,282 ; \mathrm{P}=0.0003)$. Multivariate testing of the 
GLI parameters also revealed a statistically significant diagnosis/cortical area interaction $(\mathrm{F}=1.79 ;$ d.f. $=15,768 ; \mathrm{P}=0.032)$, as well as significant dependence on cortical area alone $(\mathrm{F}=23.3$; d.f. $=15,768 ; \mathrm{P}<0.0001)$ and on lamina $(\mathrm{F}=11.8 ;$ d.f. $=5,278 ; \mathrm{P}<0.0001)$. None of $\mathrm{D}, \mathrm{sdD}, \mathrm{W}, \mathrm{sd} \mathrm{W}$, or $\mathrm{A}$, however, individually varied with the diagnosis/cortical area interaction with any significance. Regarding the distribution of interneuronal distances, multivariate tests were again significant for the interaction of diagnosis and cortical area $(\mathrm{F}=3.52$; d.f. $=6,562 ; \mathrm{P}=0.0020)$, and additionally diagnosis alone $(\mathrm{F}=4.45 ; \mathrm{d} . \mathrm{f} .=2$, $281 ; \mathrm{P}=0.012)$, cortical area alone $(\mathrm{F}=76.3$; d.f. $=6,562 ; \mathrm{P}<0.0001)$, lamina $(\mathrm{F}=17.0$; d.f. $=2,281 ; \mathrm{P}<0.0001)$, and cortical area/lamina interaction $(\mathrm{F}=4.61$; d.f. $=6,562$; $\mathrm{P}=0.0001)$. The near and far mean distances each varied significantly with diagnosis/corti$\mathrm{cal}$ area interaction $(\mathrm{F}=5.38$ and 5.33, respectively; d.f. $=3,282 ; \mathrm{P}=0.0013$ and 0.0014 , respectively), although no significant diagnosis main effect was found in either parameter.

The potential for distortion due to heating the tissue for antigen retrieval was considered. Samples of the four cortical areas of interest were obtained from both microwavetreated and untreated blocks of tissue from four patients. One sample was accidentally destroyed, leaving 15 pairs of treated/untreated tissue samples. Computerized image analysis of lamina III produced estimates of the Boolean model parameters. Matched pairs testing found no significant effect on $\bar{A}(t=1.42$; d.f. $=15 ; P=0.0878)$ or $\lambda(t=0.764 ;$ d.f. $=15$; $\mathrm{P}=0.228)$ due to heating, although there was a significant effect on $\bar{U}(\mathrm{t}=3.17$; d.f. $=15$; $P=0.0032$ ). Since a diagnosis-dependence was found in $\lambda$ only, microwaving the tissue is unlikely to have confounded the results.

\section{Discussion}

In this study of post mortem brain tissue obtained from schizophrenic subjects, we demonstrated morphometric changes consistent with altered neocortical neuropil. Indices of intra- and intercluster cellular density varied systematically across all sampled areas according to their relative position along the anteroposterior axis. These data extend long-established findings that the neocortex can be regionally parcellated according to cytoarchitectonic criteria, and confirm that the criteria used in our sampling methods were valid. Diagnosisby-area interactions were derived for cell intensity modeled as $\lambda$, a parameter incorporating local particle density and surface area according to the Boolean method for stereologi$\mathrm{cal}$ analysis of periodic gradient textures (Hahn et al., 1999). Regional values for $\lambda$ were consistent with cell density values established previously in humans and monkeys with stereological cell-counting methods (Selemon, 2001). Values obtained for $\lambda$ reflected areaspecific cytoarchitectonics i.e. increased granular cell density in striate cortex compared with lower density in area 4 (Beaulieu et al., 1992; DeFelipe et al., 2001). The difference in area values of $\lambda$ between schizophrenic and matched samples varied systematically along the caudorostral axis. Values for $\lambda$ were decreased in areas 17 and 3 and increased in areas 
4 and 9 (Figure 2) relative to matched samples. This finding suggests that earlier reports (Selemon et al., 1995, 1998) of increased cell density in dorsolateral prefrontal cortex in schizophrenics may reflect an accentuation of morphogen patterning gradients over the full rostrocaudal extent of the telencephalic vesicular surface prior to or concurrent with areaspecific cytoarchitectural development.

These data complement previous findings by Selemon and colleagues $(1995,1998)$, obtained by direct cell counting, which demonstrated neuronal density increases of $17 \%$ in area $9,10 \%$ in area 17 , and $21 \%$ in area 46 . In view of findings in schizophrenic brains of increased neuronal density and preservation of total neocortical cell count (Pakkenberg, 1993; Selemon et al., 1998), numerous histopathologic and imaging studies documenting cortical volume reduction of $3 \%$ to $13 \%$ suggest a relative decrease of neuropil in the neocortex of schizophrenics (Selemon and Goldman-Rakic, 1999). To further assess relative proportions of somal area and neuropil, we used an automated imaging method to determine the gray level index (GLI), the area percentage of Nissl-stained perikarya (Wree et al., 1982; Schleicher et al., 2005), for tissue in each sample area. Our results indicated an area-by-diagnosis interaction consistent with those obtained by Vogeley and colleagues (2003) who used a similar method to determine GLI in area 10 of schizophrenics' prefrontal cortex. This group observed decreased GLI in all layers, possibly reflecting increased neuropil or a decreased perikaryal fraction. This finding conflicts with Selemon and coworkers' $(1995,1998)$ stereological data suggesting reduced neuropil in prefrontal areas. Relevant to this finding, the GLI method provides an index for image area percentage of Nissl-stained material above an intensity threshold determined by the model and thus does not provide direct information concerning neuronal density within that area.

In order to investigate this discrepancy, we employed the Delaunay triangulation method to quantify changes in the morphometry of core radial arrays of pyramidal cells and their peripheral neuropil. This method generated a mixed bimodal distribution comprising short inter-particle distances determining mean cell spacing within minicolumnar pyramidal cell cores and long inter-particle distances indicative of the width of neuropil space between cores. Data obtained from brains of our schizophrenic cohort exhibited area-by-diagnosis interactions and a caudorostral gradient of progressively decreased values for distribution means of distances between Nissl-stained particles. These results confirm those of previous studies (Selemon, 2001) showing a comparable caudorostral trend toward increased overall neocortical density in brains of schizophrenics.

Greater reduction in the distribution modes for intra-cluster relative to inter-cluster distances was observed in rostral neocortical Brodmann areas 4 and 9 (Table 2, Table 3). The degree of reduction of mean intercluster distances in the Delaunay model corresponds to the decrease in cortical plate thickness of Brodmann area 9 in schizophrenics (Selemon et al. 2003). Reduction of peripheral neuropil by this measure can thus be accounted for by laminar thinning rather than decreased peripheral neuropil width. A recent study (Casanova et al., 2005) conducted on the same brain tissue series as analyzed in the present investigation demonstrated a significant dependence of mean cell spacing on diagnosis interactions with 
area and lamina. However, there was no significant diagnosis effect for parameters of minicolumnar width or vertical orientation of minicolumnar components. The relatively greater degree of decrease in mean intracluster distance, i.e. the Delaunay parameter corresponding to mean distance between cells within the pyramidal cell core, is consistent with previously documented size reduction of basal dendritic arbors contributing to both core and peripheral neuropil volume (Kalus et al., 2000; Broadbelt et al., 2002; Black et al., 2004), core pyramidal somata providing them metabolic support (Rajkowska et al., 1998; Pierri et al., 2001; Cotter et al., 2002) as well as apposed astroglia (Rajkowska et al., 1998). Astroglial cell death (contributing to reduced GLI) and reduced neuronal size without cell death is evident in dorsolateral prefrontal cortex of schizophrenics (Cotter et al., 2002); these results reconcile findings in prefrontal areas of increased cell density with those of reduced GLI.

Minicolumnar morphometry may be constrained by developmental processes (i.e., coordinated migration of neuronal cohorts), mechanical factors (i.e., gyrification and white matter tension effects) or functional constraints (i.e., macrocolumnar organization). Changes in mean cell spacing within its radial framework likely reflect alterations in peripheral neuropil of intracolumnar circuit features including dendritic arborization, synaptopil and networks of astroglia and inhibitory interneuron connections. These elements are largely distributed in and constitute the bulk of peripheral neuropil. Tangentially oriented basilar dendritic processes, axon collaterals and their synaptic connections also provide the network of connections characteristic of each lamina. The finding of decreased mean cell spacing in this report complements our earlier study demonstrating preserved minicolumnar morphometry by describing changes attributable to the peripheral neuropil compartment.

Numerous reports in recent years have documented schizophrenia-related qualitative/ quantitative alterations of neuropil components, varying systematically according to layer and area. In dorsolateral prefrontal cortex (area 46), significantly increased neuronal density was identified specific to layers II/III, IV, and VI (Selemon, 1998). Corresponding layer-specific reductions were demonstrated in pyramidal cell spine density (Glantz and Lewis, 2000; Kolluri et al. 2005), GABA-related gene transcripts and molecules (Pierri et al., 1999; Hashimoto et al., 2003) as well as molecules related to synaptic development and transmission (Eastwood and John, 2004). In area 9, a reduced-width layer $V$ yielded decreased immunoreactivity for GFAP, suggesting a diminished density/volume of glial processes in this region (Rajkowska et al., 2002). Decreased glial numbers have been documented in prefrontal and motor (Benes et al., 1986) as well as anterior cingulate (Stark et al., 2004) cortices. Less glia also provide diminished metabolic support for other neuropil components thereby amplifying neuropil loss. These reductions in laminar width may account for the increased pyramidal cell density in minicolumn cores whose widths are unaltered in schizophrenia. The central role of neuropil components in the pathology of schizophrenia is emphasized by the results of microarray studies which implicate regional alterations of mRNA expression for molecules involved in synaptic transmission (Mirnics et al., 2001). Linkage and association studies have identified the largest proportion of schizophrenia-related genes to be involved in mechanisms regulating synaptic development and plasticity (Harrison and Weinberger, 2005). 
Mesocortical monoaminergic projections constitute a significant component of neuropil and serve to modulate glutamatergic and GABAergic output (Seamans and Yang, 2004). These projections play a central role in regulating cell arrays subserving working memory (Yang et al., 1999; Gao and Goldman-Rakic, 2003) in dorsolateral prefrontal cortical areas affected in schizophrenia. Catecholaminergic and serotonergic fibers and receptor classes have characteristic area- and layer-specific distributions (Verney, 2003). Innervation of frontal areas in primates by dopaminergic axons exhibits layer-specific postnatal development which anticipates the specific pattern of dopamine receptor expression (Lidow and Rakic, 1992) and synaptic growth and regression in these areas (Rosenberg and Lewis, 1995; Rakic et al. 1996). Middle layers exhibiting greatest relative changes in dopamine innervation and synaptogenesis are those with notable width-reduction in schizophrenia. Axons and varicosities immunolabeled for tyrosine hydroxylase show areaand layer-specific reductions in schizophrenic cases (Akil et al., 1999). Monoaminergic brainstem nuclei develop within the first trimester in humans, and their projections extend through the basal forebrain and ramify tangentially throughout neocortex by midgestation (Nobin and Björklund, 1973; Verney et al., 1993). Monoaminergic innervation is concurrent with emergence of cortical plate lamination from 20 to 30 weeks of gestation in humans (Levitt, 2003). Thus developmental perturbations of monoaminergic nuclei during the first two trimesters may disrupt corticogenesis. Activity of aminergic and cholinergic projections modulates multiple signaling mechanisms thereby influencing early patterning and neuronal specification events. Within the context of a given stochastic and epigenetic history, such activity influences the cascade of cortical developmental events. Data generated from perinatal focal ablation of mesocortical dopamine projections resulted in layer- and area-specific reductions, including cortical width in rodents (Onténiente et al., 1980), decreased dendritic branching (Kalsbeek et al., 1987) and layer-specific reduction of dendritic length and spine densities (Felten et al., 1982).

The neocortex is an assemblage of interdependent modules. Within each module connectivity between its internal elements far exceeds connectivity between homologous modules (Casanova et al., 2003). This bias in the way that neurons connect to each other engenders a canonical circuit with massive ion-gated excitatory connections flowing through vertical strips of the cortex (Braitenberg and Schüz, 1998; Douglas and Martin, 2004). Researchers have traced the developmental transformation of ontogenetic columns into mature elements (Krmpotić-Nemanić et al., 1984; Casanova et al., 2006b). It may be that the early scaffolding of neuronal precursors along radial glia projections during corticogenesis provides the opportunity for close contact and future Hebbian reciprocity. Unsurprisingly neurons with similar stimulus selection are found in close proximity to each other (Reich et al., 2001). Therefore, the minicolumn is a possible way of compartmentalizing neurons into a vertical component that shortens both neuronal processes and conduction times.

Perpendicular to the vertical axis of information processing, slower-onset, second messenger systems provide a modulatory effect on minicolumns, i.e., cholinergic, histaminergic, serotonergic, noradrenergic, and dopaminergic systems. These modulatory systems origi- 
nate from groups of neurons that primarily employ small amines to bypass the thalamus and directly innervate large portions of the cortex. The terminal zones of these systems comprise an element of the horizontal or laminar organization of the cortex. Both cytoarchitectonic and myeloarchitectonic studies have used different lamination parameters to define anatomical regions of the cortex. However, the resultant parcellation schemes do not necessarily coincide with the innervation of individual neurochemical systems (Levitt, 2003).

Our results suggest that in schizophrenia the channels that provide for information processing within the neocortex, i.e., minicolumns, are normal. Rather, abnormalities are present in lamina where modulator systems relate information processing to behavioral states, arousal, attention, mood and motivation (Mesulam, 2000). The result may be an alteration in the "state" of information processing manifesting irself as a wide array of manifestations that characterize schizophrenia as a syndrome.

\section{Acknowledgements}

The authors wish to thank S. Cleven, J. Mueller, and A. Sund for their excellent technical assistance, L. de Zeeuw for microscopy, and M. Schüler and G. Ulmar for assessing the clinical notes of the patients investigated in this study. This article is based upon work supported by the Stanley Medical Research Foundation (H.H., C.S., and M.F.C.), the National Alliance for Autism Research (M.F.C.), and NIMH grants MH61606 (M.F.C.), MH62654 (M.F.C.), and MH69991 (M.F.C).

\section{References}

Akil, M., Pierri, J.N., Whitehead, R.E., Edgar, C.L., Mohila, C., Sampson, A.R., Lewis, D.A., 1999. Laminaspecific alterations in the dopamine innervation of the prefrontal correx in schizophrenic subjects. American Journal of Psychiatry 156, 1580-1589

Beaulieu, C., Kisvarday, Z.F., Somogyi, P., Cynader, M.S., Cowel, A., 1992. Quantitative distribution of GABAimmunopositive and -immunonegative neurons and synapses in the monkey striate cortex (area 17). Cerebral Cortex 2, 295-309

Benavides-Piccione, R., Arellano, J.I., DeFelipe, J., 2005. Catecholaminergic innervation of pyramidal neurons in the human temporal cortex. Cerebral Cortex 15, 1584-1591

Benes, F.M., Davidson, J., Bird, E.D., 1986. Quantitative cytoarchitectural studies of the cerebral cortex of schizophrenics. Archives of General Psychiarry 43, 31-35

Benes, F.M., Taylor, J.B., Cunningham, M.C., 2000. Convergence and plasticity of monoaminergic systems in the medial prefrontal cortex during the postnatal period: Implications for the development of psychopathology. Cerebral Cortex 10, 1014-1027 
Berger, B., Trottier, S., Verney, C., Gaspar, P., Alvarez, C., 1988. Regional and laminar distribution of the dopamine and serotonin innervation in the macaque cerebral cortex: A radioautographic study. Journal of Comparative Neurology 273, 99-119

Black, J.E., Kodish, I.M., Grossman, A.W., Klintsova, A.Y., Orlovskaya, D., Vostrikov, V., Uranova, N., Greenough, W.T., 2004. Pathology of layer V pyramidal neurons in the prefrontal cortex of patients with schizophrenia. American Journal of Psychiatry 161, 742-744

Braak, H., Braak, E., 1995. Staging of Alzheimer's disease-related neurofibrillary changes. Neurobiology of Aging $16,271-278$

Braitenberg, V., Schüz, A., 1998. Cortex: Statistics and Geometry of Neuronal Connectivity. Springer-Verlag, Berlin

Broadbelt, K., Byne, W., Jones, L.B., 2002. Evidence for a decrease in basilar dendrites of pyramidal cells in schizophrenic medial prefrontal cortex. Schizophrenia Research 58, 75-81

Brodmann, K., 1909. Vergleichende Lokalisationslehre der Großhirnrinde: in ihren Prinzipien dargestellt auf Grund des Zellenbaues. Barth, Leipzig

Buxhoeveden, D.P., Casanova, M.F., 2002. The minicolumn hypothesis in neurosciences. Brain 125, 935-951

Carpenter, M., 1985. Core Text of Neuroanatomy. Williams and Wilkins, Baltimore

Casanova, M.F., Buxhoeveden, D., Gomez, J., 2003. Disruption in the inhibitory architecture of the cell minicolumn: Implications for autism. The Neuroscientist 9, 496-507

Casanova, M.F., de Zeeuw, L., Switala, A., Kreczmanski, P., Korr, H., Ulfig, N., Heinsen, H., Steinbusch, H.W.M., Schmitz, C., 2005. Mean cell spacing abnormalities in the neocortex of patients with schizophrenia. Psychiatry Research 133, 1-12

Casanova, M.F., van Kooten, I.A.J., Switala, A.E., van Engeland, H., Heinsen, H., Steinbusch, H.W.M., Hof, P.R., Trippe, J., Stone, J., Schmitz, C., 2006a. Minicolumnar abnormalities in autism. Acta Neuropathologica, in press. [Epub ahead of print]

Casanova, M.F., Trippe, J., Switala, A., 2006b. A temporal continuity to the vertical organization of the human neocortex. Cereb Cortex, in press. [Epub ahead of print]

Cotter, D., Mackay, D., Chana, G., Beasley, C., Landau, S., Everall, I.P., 2002. Reduced neuronal size and glial cell density in area 9 of the dorsolateral prefrontal cortex in subjects with major depressive disorder. Cerebral Cortex $12,386-394$

DeFelipe, J., Arellano, J.I., Gómez, A., Azmitia, E.C., Murioz, A., 2001. Pyramidal cell axons show a local specialization for GABA and 5-HT inputs in monkey and human cerebral cortex. Journal of Comparative Neurology 433, 148-155

Douglas, R.J., Martin, K.A.C., 2004. Neuronal circuits of the neocortex. Annual Review of Neuroscience 27, $419-451$

Eastwood, S.L., John, S., 2004. The synaptic pathology of schizophrenia: Is aberrant neurodevelopment and plasticity to blame? International Review of Neurobiology 59, 47-72

Economo, C. von, Koskinas, G.N., 1925. Die Cytoarchitek der Hirninde des erwachsenen Menschen. Julius Springer, Wein

Felten, D.L., Hallman, H., Jonsson, G., 1982. Evidence for a neurotropic role of noradrenaline neurons in the postnatal development of rat cerebral cortex. Journal of Neurocytology 11, 119-135

Gao, W.J., Goldman-Rakic, P.S., 2003. Selective modulation of excitatory and inhibitory microcircuits by dopamine. Proceedings of the National Academy of Sciences of the United States of America 100, 2836-2841 
Glant, L., Lewis, D., 2000. Decreased dendritic spine density on prefrontal cortical pyramidal neurons in schizophrenia. Archives of General Psychiatry 57, 65-73

Hahn, U., Micheletri, A., Pohlink, R., Stoyan, D., Wendrock, H., 1999. Stereological analysis and modelling of gradient structures. Journal of Microscopy 195, 113-124

Harrison, P.J., Weinberger, D.R., 2005. Schizophrenia genes, gene expression, and neuropathology: On the matter of their convergence. Molecular Psychiatry 10, 40-68

Hashimoto, T., Volk, D.W., Eggan, S.M., Mirnics, K., Pierri, J.N., Sun, Z., Sampson, A.R., Lewis, D.A., 2003. Gene expression deficits in a subclass of GABA neurons in the prefrontal cortex of subjects with schizophrenia. Journal of Neuroscience 23, 6315-6326

Heinsen, H., Heinsen, Y.L., 1991. Serial thick, frozen, gallocyanin stained sections of human central nervous system. Journal of Histotechonology 14, 167-173

Heinsen, H., Rüb, U., Bauer, M., Ulmar, G., Bethke, B., Schüler, M., Böcker, F., Eisenmenger, W., Gö̈z, M., Korr, H., Schmitz, C., 1999. Nerve cell loss in the thalamic mediodorsal nucleus in Huntington's disease. Acta Neuropathologica 97, 613-622

Heinsen, H., Arzberger, T., Schmitz C., 2000. Celloidin mounting (embedding without infiltration): A new, simple and reliable method for producing serial sections of high thickness through complete human brains and its application to stereological and immunohistochemical investigations. Journal of Chemical Neuroanatomy 20, 49-59

Kalsbeek A., Buijs, R.M., Hofman, M.A., Matthijssen, M.A.H., Pool, C.W., Uylings, H.B.M., 1987. Effects of neonatal thermal lesioning of the mesocortical dopaminergic projection on the development of the rat prefrontal cortex. Brain Research 429, 123-132

Kalus, P., Müller, T.J., Zuschratter, W., Senitz, D., 2000. The dendritic architecture of prefrontal pyramidal neurons in schizophrenic patients. Neuroreport 11, 3621-3625

Kolluri, N., Sun, Z., Sampson, A.R., Lewis, D.A., 2005. Lamina-specific reductions in dendritic spine density in the prefrontal cortex of subjects with schizophrenia. American Journal of Psychiatry 162, 1200-1202

Kostović, I., Rakic, P., 1990. Developmental history of the transient subplate zone in the visual and somatosensory cortex of the macaque monkey and human brain. The Journal of Comparative Neurology 297, 441-470

Krmpotić-Nemanić, J., Kostović, I., Nemanić, Đ., 1984. Prenatal and perinatal development of radial cell columns in the human auditory cortex. Acta Oto-laryngologica 97, 489-495

Lambe, E.K., Krimer, L.S., Goldman-Rakic, P.S., 2000. Differential postnatal development of catecholamine and serotonin inputs to identified neurons in prefrontal cortex of rhesus monkey. The Journal of Neuroscience $20,8780-8787$

Levitt, P., 2003. Structural and functional maturation of the developing primate brain. The Journal of Pediatrics 143, suppl. 1, 35-45

Lidow, M.S., Rakic, P., 1992. Scheduling of monoaminergic neurotransmitter receptor expression in the primate neocortex during postnatal development. Cerebral Cortex 2, 401-416

Mesulam, M.M., 2000. Behavioral neuroanatomy: Large-scale networks, association cortex, frontal syndromes, the limbic system, and hemispheric specializations. In: Mesulam, M.M. (Ed.), Principles of Behavioral and Cognitive Neurology, 2nd ed. Oxford University Press, Oxford, pp. 1-120

Mirnics, K., Middleton, F.A., Lewis, D.A., Levitt, P., 2001. The human genome: Gene expression profiling and schizophrenia. American Journal of Psychiatry 158, 1384

Mountcastle, V.B., 1997. The columnar organization of the neocortex. Brain 120, 701-722 
Chapter 5

Nobin, A., Björklund, A., 1973. Topography of the monoamine neuron systems in the human brain as revealed in fetuses. Acta physiologica Scandinavica: Supplementum 388

Onténiente, B., König, N., Sievers, J., Jenner, S., Klemm, H.P., Marty, R., 1980. Structural and biochernical changes in rat cerebral cortex after neonatal 6-hydroxydopamine administration. Anatomy and Embryology 159, 245-255

Otsu, N., 1979. A threshold selection method from grey-level histograms. IEEE Transactions on Systems, Man, and Cybernetics 9, 377-393

Pakkenberg, B., 1993. Total nerve cell number in neocortex in chronic schizophrenics and controls estimated using optical disectors. Biological Psychiatry 34, 768-772

Pierri, J.N., Chaudry, A.S., Woo, T.U.W., Lewis, D.A., 1999. Alterations in chandelier neuron axon terminals in the prefrontal cortex of schizophrenic subjects. American Journal of Psychiatry 156, 1709-1719

Pierri, J.N., Volk, C.L.E., Auh, S., Sampson, A.R., Lewis, D.A., 2001. Decreased somal size of deep layer 3 pyramidal neurons in the prefrontal cortex of subjects with schizophrenia. Archives of General Psychiatry 58, 466-473

Quester, R., Schroeder, R., 1997. The shrinkage of the human brain during formalin fixation and embedding in paraffin. Journal of Neuroscience Methods 75, 81-89

Rajkowska, G., Goldman-Rakic, P.S., 1995a. Cytoarchitectonic definition of prefrontal areas in the normal human cortex: I. Remapping of areas 9 and 46 using quantitative criteria. Cerebral Cortex. 5, 307-322

Rajkowska, G., Goldman-Rakic, P.S., 1995b. Cytoarchitectonic definition of prefrontal areas in the normal human cortex: II. Variability in locations of areas 9 and 46 and relationship to the Talairach Coordinate System. Cerebral Cortex. 5, 323-327

Rajkowska, G., Selemon, L.D., Goldman-Rakic, P.S., 1998. Neuronal and glial somal size in the prefrontal cortex: A postmortem morphometric study of schizophrenia and Huntington disease. Archives of General Psychiatry 55, 215-224

Rajkowska, G., Miguel-Hidalgo, J.J., Makkos, Z., Meltzer, H., Overholser, J., Stockmeier, C., 2002. Layerspecific reductions in GFAP-reactive astroglia in the dorsolateral prefrontal cortex in schizophrenia. Schizophrenia Research 57, 127-138

Rakic, P., 1988. Specification of cerebral cortical areas. Science 241, 170-176

Rakic, P., 1995. Radial glial cells: Scaffolding for brain construction. Kettenmann, H., Ransom, B.R. (Eds.), Neuroglia. Oxford University Press, New York, pp. 746-762

Rakic, P., Knyihar-Csillik, E., Csillik, B., 1996. Polarity of microtubule assemblies during neuronal cell migration. Proceedings of the National Academy of Sciences of the United States of America 93, 9218-9222

Reich, D.S., Mechler, F., Victor, J.D., 2001. Independent and redundant information in nearby cortical neurons. Science 294, 2566-2568

Rosenberg, D.R., Lewis, D.A., 1995. Postnatal maturation of the dopaminergic innervation of monkey prefrontal and motor cortices: a tyrosine hydroxylase immunohistochemical analysis. Journal of Comparative Neurology $358,383-400$

Schleicher, A., Palomero-Gallagher, N., Morosan, P., Eickhoff, S.B., Kowalski, T., de Vos, K., Amunts, K., Zilles, K., 2005. Quantitative architectural analysis: A new approach to cortical mapping. Anatomy and Embryology 210, 373-386

Seamans, J.K., Yang, C.R., 2004. The principal features and mechanisms of dopamine modulation in the prefrontal cortex. Progress in Neurobiology 74, 1-58 
Selemon, L.D., Rajkowska, G., Goldman-Rakic, P.S., 1995. Abnormally high neuronal densiry in the schizophrenic cortex: A morphometric analysis of prefrontal area 9 and occipital area 17. Archives of General Psychiatry 52, 805-820

Selemon, L.D., Rajkowska, G., Goldman-Rakic, P.S., 1998. Elevated neuronal density in prefrontal area 46 in brains from schizophrenic patients: Application of a three-dimensional, stereologic counting method. Journal of Comparative Neurology 392, 402-412

Selemon, L.D., Goldman-Rakic, P.S., 1999. 'The reduced neuropil hypothesis: A circuit based model of schizophrenia. Biological Psychiatry 45, 17-25

Selemon, L.D. 2001.Regionally diverse cortical pathology in schizophrenia: Clues to the exiology of the disease. Schizophrenia Bulletin 27, 349-377

Selemon L.D., Mrzljak J., Kleinman J.E., Herman M.M., Goldman-Rakic P.S., 2003. Regional specificity in the neuropathologic substrates of schizophrenia: a morphometric analysis of Broca's area 44 and area 9. Archives of General Psychiatry 60, 69-77

Stark, A.K., Uylings, H.B.M., Sanz-Arigita, E., Pakkenberg, B., 2004. Glial cell loss in the anterior cingulate cortex, a subregion of the prefrontal cortex, in subjects with schizophrenia. American Journal of Psychiatry $161,882-888$

Stoyan, D., Kendall, W.S., Mecke, J., 1995. Stochastic Geometry and its Applications, 2nd ed. John Wiley and Sons, Chichester

Verney, C., Milosevic, A, Alvarez, C., Berger, B., 1993. Immunocytochemical evidence of well-developed dopaminergic and noradrenergic innervations in the frontal cerebral cortex of human fetuses at midgestation. Journal of Comparative Neurology 336, 331-344

Verney, C., 2003. Phenotypic expression of monoamines and GABA in the early development of human telencephalon, transient or not transient. Journal of Chemical Neuroanatomy 26, 283-292

Vogeley, K., Tepest, R., Schneider-Axmann, T., Hütte, H., Zilles, K., Honer, W.G., Falkai, P., 2003. Automated image analysis of disturbed cytoarchitecture in Brodmann area 10 in schizophrenia. Schizophrenia Research $62,133-140$

Vogt, C., Vogt, O., 1919. Allgemeinere Ergebnisse unserer Hirnforschung: dritte Mitteilung. die architektonische Rindenfelderung im Lichte unserer neuesten Forschungen. Journal für Psychologie und Neurologie 25, 361-376

Wree, A., Schleicher, A., Zilles, K., 1982. Estimation of volume fractions in nervous tissue with an image analyzer. Journal of Neuroscience Methods 6, 29-43

Yang, C.R., Seamans, J.K., Gorelova, N., 1999. Developing a neuronal model for the pathophysiology of schizophrenia based on the nature of electrophysiological actions of dopamine in the prefrontal cortex. Neauropsychopharmacology 21, 161-194 


\section{Tables}

Table 1. Clinical characteristics of the cases included in this study. PMl: interval between death and autopsy. Storage: duration of immersion in $10 \%$ formalin. All schizophrenic patients and all controls were male.

\begin{tabular}{|c|c|c|c|c|c|c|}
\hline \multirow{2}{*}{ No. } & \multirow{2}{*}{$\begin{array}{c}\begin{array}{c}\text { Age } \\
\text { [years] }\end{array} \\
\end{array}$} & \multirow{2}{*}{ Cause of death } & \multirow{2}{*}{ PMI [hours] } & \multirow{2}{*}{$\begin{array}{c}\text { Storage } \\
\text { [days] }\end{array}$} & \multicolumn{2}{|c|}{ Diagnosis } \\
\hline & & & & & DSM-IV & $I C D-10$ \\
\hline S1 & 22 & Suicide & $88^{1}$ & 130 & 295.30 & $F 20.0^{2}$ \\
\hline 52 & 36 & Suicide & $<72^{\prime}$ & 115 & 295.30 & $F 20.0^{2}$ \\
\hline 53 & 46 & Systemic hypothermia & $<24$ & 327 & 295.30 & $F 20.01^{2}$ \\
\hline S4 & 50 & Peritonitis & $<24$ & 203 & 295.30 & $\mathrm{~F} 20.0^{2}$ \\
\hline S5 & 50 & Suicide & 18 & 170 & 295.30 & $F 20.0^{2}$ \\
\hline S6 & 51 & Septicemia & 33 & 127 & 295.60 & $F 20.50^{3}$ \\
\hline 57 & 54 & Septicemia & 27 & 250 & 295.60 & $F 20.50^{3}$ \\
\hline 58 & 55 & Right-sided heart failure & 25 & 84 & 295.30 & $F 20.00^{2}$ \\
\hline 59 & 57 & Septicemia & 76 & 163 & 295.30 & $F 20.00^{2}$ \\
\hline $\mathrm{S} 10$ & 60 & Pulmonary embolism & $<48$ & 311 & 295.30 & $F 20.01^{2}$ \\
\hline S11 & 62 & Aspiration & 7 & 171 & 295.30 & $F 20.00^{2}$ \\
\hline $\mathrm{S} 12$ & 63 & Acute myocardial infarct & 15 & 338 & 295.60 & $F 20.50^{3}$ \\
\hline$\$ 13$ & 64 & Pulmonary embolism & 6 & 817 & 295.60 & $F 20.50^{3}$ \\
\hline $\mathrm{Cl}$ & 25 & Cardiac tamponade & 14 & 119 & & \\
\hline$C_{2}$ & 36 & Gunshot & 24 & 143 & & \\
\hline $\mathrm{C} 3$ & 47 & Acute myocardial infarct & $<24$ & 133 & & \\
\hline $\mathrm{C} 4$ & 50 & Acute myocardial infarct & 35 & 433 & & \\
\hline $\mathrm{C5}$ & 50 & Avalanche accident & 23 & 498 & & \\
\hline C6 & 51 & Septicemia & 7 & 285 & & \\
\hline $\mathrm{C7}$ & 54 & Acute myocardial infarct & 18 & 168 & & \\
\hline $\mathrm{C} 8$ & 56 & Acute myocardial infarct & 60 & 3570 & & \\
\hline $\mathrm{Cg}$ & 58 & Acute myocardial infarct & 28 & 126 & & \\
\hline $\mathrm{C} 10$ & 60 & Gastrointestinal hemorrhage & 18 & 101 & & \\
\hline $\mathrm{C} 11$ & 60 & Gastrointestinal hemorrhage & 27 & 302 & & \\
\hline $\mathrm{C} 12$ & 62 & Acute myocardial infarct & $<24$ & 3696 & & \\
\hline $\mathrm{C} 13$ & 65 & Bronchopneumonia & 6 & 2289 & & \\
\hline
\end{tabular}

These patients had long post mortem intervals (PMI). However, both patients committed suicide (one by hanging, the other by jumping from an eight story building), were found within $1 \mathrm{~h}$ of death, and were kept at $4^{\circ} \mathrm{C}$ until autopsy. Accordingly, the intervals between death and autopsy of these patients cannot be compared to the corresponding intervals of the other persons and were thus excluded from the calculation of the mean PMI between death and autopsy.

2Paranoid schizophrenia.

${ }^{3}$ Residual schizophrenia.

PMl: interval between death and autopsy. Storage: duration of immersion in $10 \%$ formalin. All schizophrenic patients and all controls were male. 
Table 2. Mean values of those measurements with joint dependence on diagnosis and cortical area. S: schizophrenic group; C: normal control group. The mean of both laminae investigated is given. To obtain values specific to lamina III (respectively, lamina V), subtract (resp., add) $179 \mathrm{~mm}^{-2}$ to $\lambda$, and multiply (resp., divide) $m_{\text {near }}$ by 1.016. $m_{\text {far }}$ does not differ significantly by lamina. S.D. is the Residual root mean squared error, or pooled standard deviation. Standard deviations for interneuronal distances are given as percentages of the mean value.

\begin{tabular}{|c|c|c|c|c|c|c|c|c|}
\hline \multirow[b]{2}{*}{ Brodmann area } & \multicolumn{2}{|c|}{$\lambda\left(\mathrm{mm}^{-2}\right)$} & \multicolumn{2}{|c|}{$m_{\text {near }}(\mu \mathrm{m})$} & \multicolumn{2}{|c|}{$m_{\text {far }}(\mu \mathrm{m})$} & \multicolumn{2}{|c|}{$m_{\text {far }}-m_{\text {near }}(\mu \mathrm{m})$} \\
\hline & $S$ & $C$ & $S$ & $C$ & $S$ & $C$ & $\mathbf{S}$ & $C$ \\
\hline$\uparrow-9$ & 1416 & 1308 & 24.3 & 25.2 & 48.3 & 49.8 & 24.0 & 24.6 \\
\hline$\frac{1}{2} 4$ & 1313 & 1291 & 25.1 & 25.7 & 51.0 & 50.9 & 25.9 & 25.2 \\
\hline$\stackrel{\omega}{n}_{3}{ }_{3}$ & 1673 & 1867 & 24.2 & 23.6 & 48.2 & 46.5 & 24.0 & 22.9 \\
\hline$\stackrel{\propto}{\simeq} \downarrow 17$ & 2838 & 3230 & 20.8 & 20.0 & 40.3 & 38.3 & 19.5 & 18.3 \\
\hline S.D. & \multicolumn{2}{|c|}{387} & \multicolumn{2}{|c|}{$6.87 \%$} & \multicolumn{2}{|c|}{$6.98 \%$} & \multicolumn{2}{|c|}{$10.36 \%$} \\
\hline
\end{tabular}

Table 3. 95\% confidence intervals on the mean values in Table 2. The same remarks apply regarding values in lamina III versus lamina $\mathrm{V}$.

\begin{tabular}{|c|c|c|c|c|c|c|c|}
\hline \multirow{2}{*}{\multicolumn{2}{|c|}{ Brodmann area }} & \multicolumn{2}{|c|}{$\lambda\left(\mathrm{mm}^{2}\right)$} & \multicolumn{2}{|c|}{$m_{\text {near }}(\mu \mathrm{m})$} & \multicolumn{2}{|c|}{$m_{f o r}(\mu \mathrm{m})$} \\
\hline & & $S$ & C & $\mathrm{s}$ & C & $\mathrm{S}$ & C \\
\hline \multirow{4}{*}{ 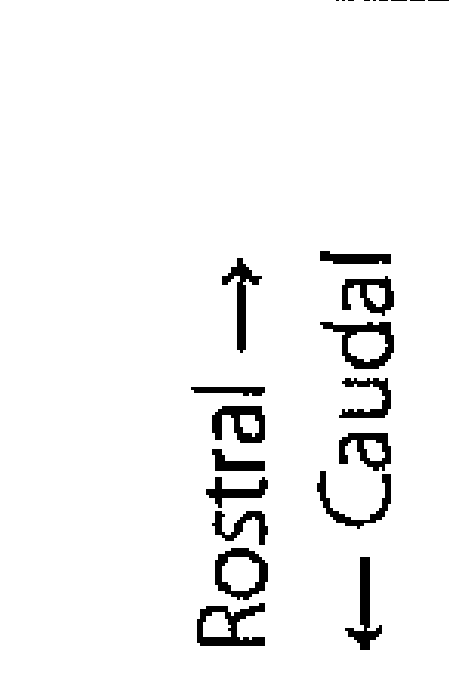 } & 9 & {$[1317,1514]$} & {$[1215,1404]$} & {$[23.9,24.8]$} & {$[24.7,25.6]$} & {$[47.5,49.2]$} & {$[48.9,50.6]$} \\
\hline & 4 & {$[1216,1406]$} & {$[1185,1398]$} & {$[24.7,25.5]$} & {$[25.3,26.2]$} & {$[50.2,51.9]$} & {$[49.9,51.9]$} \\
\hline & 3 & {$[1578,1767]$} & {$[1740,1990]$} & {$[23.8,24.7]$} & {$[23.1,24.1]$} & {$[47.4,49.1]$} & {$[45.5,47.5]$} \\
\hline & 17 & {$[2742,2933]$} & {$[3119,3342]$} & {$[20.4,21.1]$} & {$[19.6,20.4]$} & {$[39.6,41,0]$} & {$[37.6,39.1]$} \\
\hline
\end{tabular}




\section{Figures}

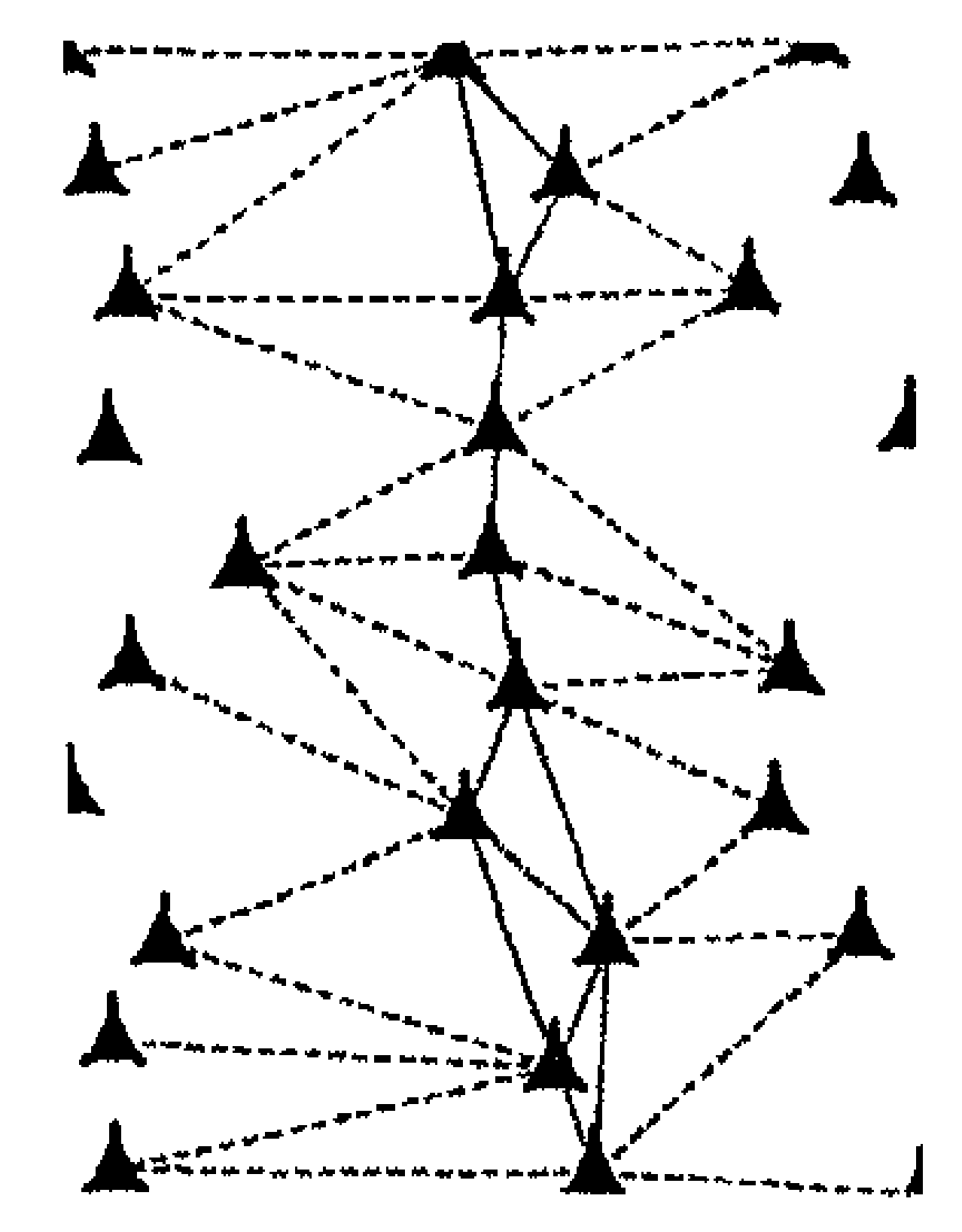

Fig. 1. In this schematic, a linear cluster (minicolumnar fragment) is situated between two neighboring clusters. Cells, whether in the same cluster or no, are considered adjacent if they are joined by an edge of the Delaunay triangulation of cell centroids. (Three points comprise the vertices of a Delaunay triangle if and only if the circle through those points has no other point in its interior.) Delaunay edges between cells within a cluster (solid lines) will tend to be shorter than edges between cells in different clusters (broken lines). Not knowing a priori which cells belong to which cluster, one can estimate the intracluster and intercluster distances by considering the lengths of edges to be distributed according to a two-component mixture model.

Edges of the triangulation not involving any of the points of the middle cluster in the diagram have been omitted for clarity.

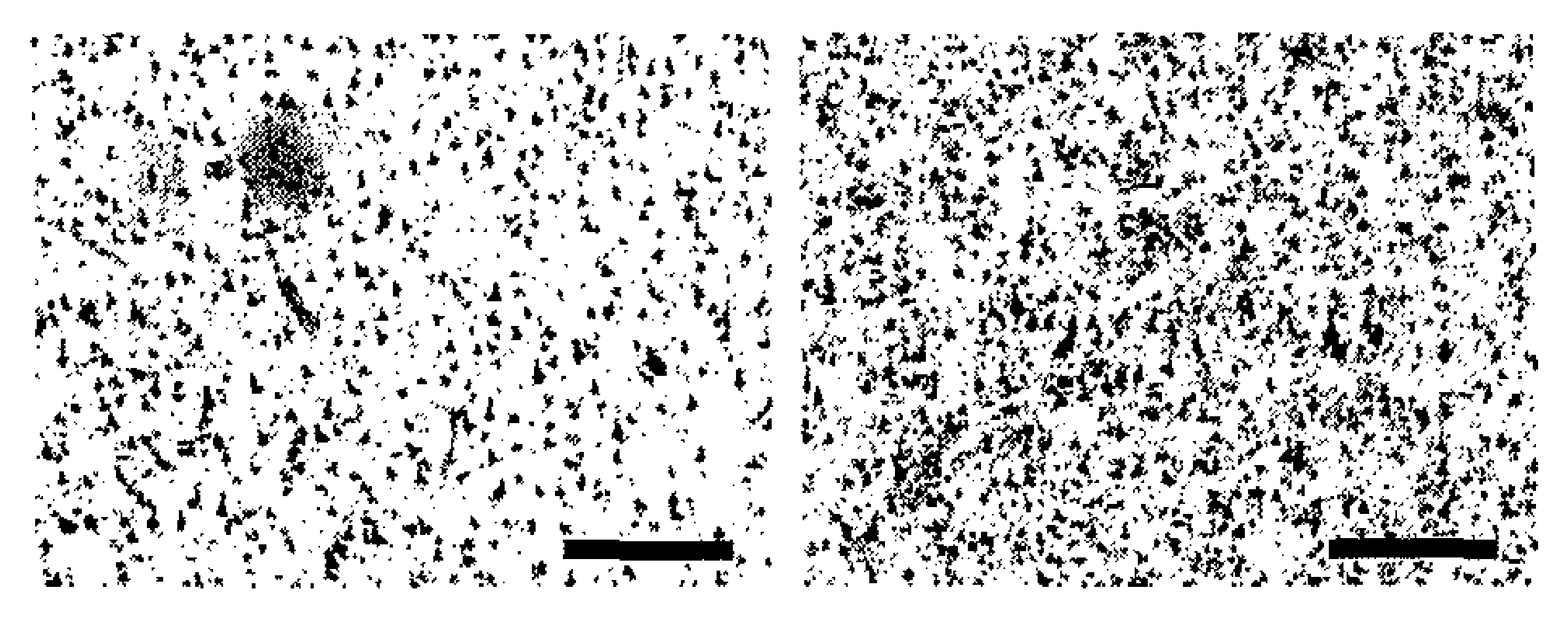

Fig. 2. Increased cell density can be seen in these micrographs of prefrontal area 9 (right hemisphere, lamina III). In the normal patient (left), estimated Boolean model parameters were $\bar{A}=85.8 \mu \mathrm{m}^{2}, \bar{U}=31.6 \mu \mathrm{m}$, and $\lambda=823 \mathrm{~mm}^{-2}$. In the schizophrenic patient (right), $\bar{A}=73.4 \mu \mathrm{m}^{2}$, $\bar{U}=29.4 \mu \mathrm{m}$, and $\lambda=1640 \mathrm{~mm}^{-2}$. Scalebars measure $250 \mu \mathrm{m}$. 


\section{CMCpter 6}

\section{Mean cell spacing abnormalities in the neocortex of patients with schizophrenia}

Manuel F. Casanova $a^{\mathrm{a},{ }^{*},}$, Leonie de Zeeuw ${ }^{\mathrm{b}}$, Andrew Switala Pawel Kreczmanski ${ }^{b, c, d, e, f}$, Hubert Korre,f, Norbert Ulfig ${ }^{c}$, Helmut Heinsen ${ }^{g}$ Harry W.M. Steinbusch ${ }^{\text {b,f }}$, Christoph Schmitz ${ }^{\mathrm{b}, \mathrm{c} f}$

¿Department of Psychiatry and Behavioral Sciences, University of Louisville, Louisville, KY 40292, USA

bepartment of Psychiatry and Neuropsychology, Division of Cellular Neuroscience, University of Maastricht, Maastricht, Netherlands

'Neuroembryonic Research Laboratory, Department of Anatomy, University of Rostock, Rostock, Germany

'Department of Anatomy, Center of Biostructure, Medical University of Warsaw, Warsaw, Poland

eDepartment of Anatomy and Cell Biology, RWTH Aachen Unlversity, Aachen, Germany

'European Graduate School of Neuroscience (EURON), Maastricht, Netherlands

9Morphological Brain Research Unit, University of Wuerzburg, Wuerzburg, Germany

Psychiatry Research 158 (2008) 267-277 


\begin{abstract}
It has been postulated that the prefrontal corticcs of schizophrenic patients have significant alterations in their interneuronal (neuropil) space. The present study re-examines this finding based on measurements of mean cell spacing within the cell minicolumn. The population studied consisted of 13 male schizophrenic patients (DSM-IV criteria) and 13 age-matched controls. Photomicrographs of Brodmann's areas 9, 4 (M1), 3b (S1), and $17(\mathrm{~V} 1)$ were analyzed with computerized image analysis to measure parameters of minicolumnar morphometry, i.e., columnarity index (CI), minicolumnar width (CW), dispersion of minicolumnar width (VCW), and mean interneuronal distance (MCS). The results indicate alterations in the mean cell spacing of schizophrenic patients according to both the lamina and cortical area examined. The lack of variation in the columnarity index argues in favor of a defect postdating the formation of the cell minicolumn.
\end{abstract}




\section{Introduction}

It has been noted that the prefrontal cortices of schizophrenic patients have less interneuronal (neuropil) space than controls, specifically in areas 9 and 46 (Selemon et al., 1995). This is thought to reflect an alteration in the neural circuitry of these areas and is a possible component of the pathology of schizophrenia. More recently, other researchers have used an automated image analysis method to scan the whole cortical width (Brodmann area 10) of schizophrenic patients for gray-level index (GLI), i.e., the area percentage covered by perikarya (Vogeley et al., 2003). Results of these experiments - reductions in the graylevel index of all cortical layers - were consistent with a decreased perikaryal fraction or an increased amount of neuropil. All of these studies were based on measures of neuronal density which focus on morphological alterations at the cellular level while disregarding the anatomical interdependence of neurons. The lack of analysis of the anatomical arrangement of neurons into physiological units might compromise any results from neuropathological studies. Neurons are interdependent elements within cortical modules, the smallest of which seems to be the cell minicolumn (Casanova et al., 2003; Mountcastle, 1998). Our approach takes advantage of the capabilities of computerized image analysis to procure adequate information regarding the morphology of cortical minicolumns.

\section{Methods}

\section{Brain specimens}

We investigated post mortem brains (both hemispheres) from 13 male schizophrenics with a mean age of 51.5 years (standard deviation [s.d.] 11.8 years) and a mean interval between death and autopsy of 27.6 hours (s.d. 19.9 hours). Controls consisted of 13 age-matched males with a mean age of 51.9 years (s.d. 11.1 years) and a mean interval between death and autopsy of 23.7 hours (s.d. 13.6 hours). Clinical diagnoses, causes of death and the interval between death and autopsy are summarized in Table 1. All schizophrenics were long-term patients at German university hospitals or at German state psychiatric hospitals. Complete clinical records were available in all cases. Records from autopsy (including a summary of the medical history) were available for all controls. All of the patients met the Diagnostic Statistical Manual, 4th revision (DSM-IV) and International Statistical Classification of Diseases and Related Health Problems, 10th revision (ICD-10) diagnostic criteria for schizophrenia. The clinical notes were assessed by experienced clinical psychiatrists (mentioned in Acknowledgements) to ensure that the control brains were free from psychopathology, and for clear evidence that the schizophrenic cases conformed to DSM-IV criteria for schizophrenia. In particular, the schizophrenic group included no patient diagnosed with schizoaffective disorder. Exclusion criteria comprised neurological problems that required intervention or interfered with cognitive assessment (e.g., stroke 
with aphasia), history of recurrent seizure disorder (i.e., epilepsy), history of severe head injury with loss of consciousness, diabetes mellitus with poor glycemic control (free plasma glucose $>200 \mathrm{mg} / \mathrm{dl}$ ), and history of self-induced water intoxication with hyponatremia. Brains from schizophrenics were also excluded if patients had undergone a leukotomy. The two groups - schizophrenics and controls - were similar in terms of the ethnic backgrounds of the subjects. However, they were not fully matched for socio-economical status and education, which would have placed severe constraints on our sample. Moreover, all schizophrenics were subjected to long-term treatment with high doses of neuroleptics.

In all of the cases, autopsy was performed after consent was obtained from a patient's relative according to the laws of the Federal Republic of Germany. The use of these autopsy cases for scientific investigations as outlined here has been approved by the relevant Institutional Review Boards (IRB). Brains were fixed by immersion in $10 \%$ formalin (1 part commercial $40 \%$ aqueous formaldehyde in 9 parts $\mathrm{H} 2 \mathrm{O}$ ) for at least 3 months and up to 11 years.

\section{Tissue processing}

The brainstem with the cerebellum was severed at the level of the rostral pons, and the hemispheres divided medio-sagittally. Then, both hemispheres were cut into serial coronal sections $600 \mu \mathrm{m}$ to $700 \mu \mathrm{m}$ thick coronal sections as previously described in the literature (Heinsen and Heinsen, 1991). Briefly, the hemispheres were cryoprotected in a mixture of glycerol-dimethylsulfoxide-formalin after carefully removing meninges and pial vessels. They were then embedded in gelatin, frozen at $-60^{\circ} \mathrm{C}$, and serially sectioned using a cryostat (Jung, Nussloch, Germany). One brain (control case C7) was embedded in celloidin (Heinsen et al., 2000), and cut into serial $440 \mu \mathrm{m}$ thick coronal sections using a sliding microtome (Polycut, Cambridge Instruments, UK). From each hemisphere, every second or third section was stained with gallocyanin (Heinsen and Heinsen, 1991). The remaining sections were stored in formalin for up to ten years.

From all the brains of schizophrenics and controls older than 40 years, sections through the central parts of the entorhinal and transentorhinal cortices were labeled with the Gallyas silver method to detect neurofibrillary changes (Heinsen et al., 1999). Neurofibrillary tangles were rarely detected. In affected patients the findings corresponded to Braak-Braak Alzheimer classification stage I (Braak and Braak, 1995). Furthermore, all of the sections used in this study were coded and controlled for the absence of tumors, infarcts, heterotopias, signs of autolysis, staining artifacts, and gliosis.

Gallocyanin-stained sections were used by L.d.Z., P.K. and C.S. to identify areas M1, V1, frontal association cortex (areas 4, 17, and 9 of Brodmann [1909], respectively) and S1 (area 3b of Vogt and Vogt [1919]) in both cerebral hemispheres according to anatomical landmarks and cytoarchitectural criteria. Gross anatomical landmarks for M1 include the anterior wall of the central sulcus and adjacent portions of the precentral gyrus. Cytoarchitectonically, the region is clearly demarcated by its giant Betz cells and minimization of layer IV. Brodmann area (BA) 17 (V1) is located along the walls of the calcarine sulcus in the occipital lobe and adjacent portions of the cuneus and lingual gyrus (Carpenter, 
1985). It is defined histologically by a broad lamina IV divided into 3 sublayers with numerous very small pyramidal cells in layers II and III. It is noted for the dense line of Gennari in myelin stains. Brodmann area 9 lies in the superior and middle frontal gyrus. Rajkowska and Goldman-Rakic (1995a, 1995b) found that it was located in the middle third of the superior frontal gyrus in all the cases they examined. It covered both dorsolateral and dorsomedial surfaces of gyrus and extended in some cases to the depth of the superior frontal gyrus and portions of the middle frontal gyrus.

Concerning BA 9, Rajkowska and Goldman-Rakic (1995b) showed that its more lateral portions embrace area 46 (area FD delta of Economo and Koskinas [1925]). It is important to note that Brodmann (1909) never described the cytoarchitectonic characteristics of area 9 in man. Rather he projected his observations in monkey onto the frontal lobe of man. Economo and Koskinas (1925) provided a detailed cytoarchitectonic description of the human frontal lobe. Their area FDm corresponds to BA 9. It is characterized by a distinct layer II, absence of very large, slender and well-built pyramidal cells in layer IIIc (typical of BA 6 and to a lesser extend of BA 8), and an uninterrupted layer IV with fairly definite upper and lower boundaries. In thick gallocyanin-stained sections a cell-poor layer is found between layers IIIc and layer IV. Layer IV is more prominent and cell-rich in the frontopolar regions; however the transition between BAs 9 and 10 is a gradual one. Layer $\mathrm{V}$ of BA 9 is subdivided into layer $\mathrm{Va}$ and $\mathrm{Vb}$, the latter of which is rather cell-sparse. Most probably the cell-poor layers between lamina IIIc and IV and within layer Vb correspond to the upper and lower band described by Rajkowska and Goldman-Rakic (1995a) in myelin-stained sections of BA 9. Finally, layer VI consists of sublayers VIa and VIb. $\mathrm{VIa}$ is cell-rich and its upper border to the cell-sparse layer $\mathrm{Vb}$ can easily be recognized. The lower boundaries of layer $\mathrm{Vb}$ to the underlying white matter are much more distinct than in the caudally ensuing BAs 8 and 6 . The overall cortical thickness of the frontopolar area 10 (field FE of Economo and Koskinas [1925]) is considerably less than in BA 9, lamina VIa is small, rich in darkly-stained pyramidal as well as spindle-shape cells and the boundary of VIa to the medullary layer is even more distinct than in BA 9. The presence of the small and cell rich layer VI facilitates the delineation of BA 9 from the rostral area 10 in thick gallocyanin stained sections. Finally, layer IV is more conspicuous than its corresponding part in BA 9.

Regions of interests were removed as $2 \mathrm{~cm} \times 2 \mathrm{~cm}$ tissue pieces. After rinsing in tap water for several hours, the pieces were stored in tris-buffered saline (TBS) overnight $(\mathrm{pH}=9.0)$. They were then placed in TBS $(\mathrm{pH}=9.0)$ and heated in a microwave oven for $15 \mathrm{~min}$ at $90^{\circ} \mathrm{C}$ for antigen retrieval (results of immunohistochemical analysis will be published elsewhere). After cooling down, the tissue blocks were rinsed in TBS twice $(\mathrm{pH}=7.4)$ and placed in a TBS based $30 \%$ sucrose solution for cryoprotection. The pieces were then frozen and cut into $50 \mu \mathrm{m}$ thick sections using a cryostat (Frigocut 2800E; Jung, Nussloch, Germany). These $50 \mu \mathrm{m}$ thick sections were stained with cresylviolet $(0.1 \%, 20 \mathrm{~min})$. 


\section{Computerized image analysis}

Digital images were taken from laminas III and V in cortical areas M1, S1, V1, and Brodmann area 9 in both cerebral hemispheres. Photomicrographs were taken through the flat face of the gyri with minicolumns perpendicular to the pia. Each field of view measured approximately $1.45 \mathrm{~mm} 2$, and up to three fields were captured per lamina/area/hemisphere as tissue quality allowed. Of the 416 possible regions of interest ( 16 in each of 26 brains), 90 were completely lacking in usable tissue; the number of images captured per available region numbered 2.7 .

The columnarity index, CI, a global measure of anisotropy or degree of orientation (Figure 1), was obtained with a Fourier transform algorithm as follows (Casanova and Switala, 2005): The dominant orientation of the image was found using the formula

$$
\theta_{d}=\frac{1}{2} \tan ^{-1} \frac{\sum_{i, j}\left|F_{i j}\right|^{2} \sin 2 \theta_{i j}}{\sum_{i, j}\left|F_{i j}\right|^{2} \cos 2 \theta_{i j}}
$$

where $|\mathrm{Fij}|^{2}$ is the squared amplitude and $\tan \theta i j=f_{y}(i, j) / f_{x}(i, j)$ is the orientation corresponding to the frequency component at line $i$, sample $j$ of the discrete Fourier transform of the image (Pourdeyhimi et al., 1994). Then the columnarity index was defined by

$$
C I=\sqrt{\frac{\sum_{i, j}\left|F_{i j}\right|^{2} \cos 2\left(\theta_{i j}-\theta_{\mathrm{d}}\right)}{\sum_{i, j}\left|F_{i j}\right|^{2}}}
$$

which is constrained to lie between zero and one, inclusive.

Computerized image analysis of minicolumnar structure was performed with a modification of algorithms previously described in the literature (Buxhoeveden et al., 2000a): Each image was flattened to correct for uneven illumination by first estimating and then subtracting background. Background estimation involved smoothing the image followed by filling depressions in the image viewed as an intensity surface, i.e. removing any dark regions surrounded by bright pixels. The flattened image was segmented using thresholding with Otsu's method, which maximizes the contrast between the two pixel classes (above and below threshold) with respect to the pooled within-class variance (Otsu, 1979). Nonconvex foreground (dark) patches in the binary image were subdivided by applying the morphological watershed transform to the distance map of the binary image. Excessively oblong watershed regions were identified, and corresponding basins of the distance map were filled in. Then the watershed algorithm was applied to the modified distance map. Each connected region in the resulting image was further classified as a large neuron if its area was greater than $30 \mu \mathrm{m}^{2}$ and as a small neuron if its area was between $10 \mu \mathrm{m}^{2}$ and $30 \mu \mathrm{m}^{2}$. Smaller objects, assumed to be glia, cell fragments, or noise, were discarded 
altogether. In our study small granule cells and glia that may have been omitted would not have significantly affected column location or morphology since cell columns essentially consists of pyramidal cells.

Local cell density was calculated as follows: $\mathrm{d}$ was the typical distance between neighboring large neurons. Each image was divided into horizontal bands $2 \mathrm{~d}$ high. Within each band, the observed cell density was smoothed as a function of horizontal position using a bell-shaped kernel of width (1/2)d (Figure 2). Local maxima of cell density indicated minicolumn cores, and local minima indicated peripheral neuropil space. Local maxima in each strip were joined with mutual nearest neighbors in the strips above and below to form chains. Minicolumnar width (CW) was defined as the median over all distances between chains adjacent to the left or right, and its dispersion (VCW) was the median absolute deviation of these distances. Mean interneuronal distance (MCS) was calculated as for $\mathrm{d}$, above, including all neurons large and small.

\section{Bootstrap confidence regions}

The basic bootstrap method (Davison and Hinkley, 1997) was used to compute confidence intervals on the mean MCS and CW, below. Simulated data sets were generated by resampling cases: each simulated data set was composed of the measurements from thirteen schizophrenic patients and thirteen control cases selected randomly and allowing for duplication (Table 2). These were paired as in the original data set, youngest schizophrenic with youngest control, second-youngest with second-youngest, and so forth. Approximately 10,000 bootstrap samples were thus generated. The linear model described below was fit to each artificial data set in turn and used to estimate the expected values of the dependent variables in each lamina, cortical area, and pathological condition. The orientation and eccentricities of the confidence ellipses are given by the covariance matrix of the bootstrap expected values; the size of the confidence ellipses is the least such that it encloses just over $95 \%$ of the bootstrap estimates.

\section{Results}

For purposes of statistical analysis each schizophrenic patient was paired with his agematched normal counterpart. Diagnosis, cortical area, hemisphere, and lamina were included as effects in a full factorial model. All four dependent variables (columnarity index $[\mathrm{CI}]$, minicolumnar width [CW], dispersion of minicolumnar width [VCW], and mean interneuronal distance $[\mathrm{MCS}]$ ) were analyzed simultaneously. The multivariate likelihood ratio statistics supported a relationship between the dependent variables and cortical area $\left(\chi^{2}=430,12\right.$ degrees of freedom, $\left.\mathrm{p}<0.001\right)$, lamina $\left(\chi^{2}=109,4\right.$ d.f., $\left.\mathrm{p}<0.001\right)$, diagnosis by cortical area interaction $\left(\chi^{2}=21.2,12\right.$ d.f., $\left.p=0.048\right)$, and lamina by cortical area interaction $\left(\chi^{2}=31.9,12\right.$ d.f., $\left.\mathrm{p}=0.002\right)$. Each of the four measurements, considered 
individually, varied significantly with cortical area $(\mathrm{p}<0.001)$. CI, VCW, and MCS varied significantly with lamina ( $F 1,282>22, \mathrm{p}<0.001$ in each variable), but $\mathrm{CW}$ did not $(\mathrm{F} 1,282=0.449, \mathrm{p}=0.654)$.

MCS was the only single measurement with a significant dependence on the diagnosis by cortical area interaction term $(F 3,282=3.26, p=0.022)$. There was a suggestion of greater cell packing within the minicolumn in schizophrenia in the prefrontal and motor areas but lesser packing in the primary sensory areas (Figure 3, Table 3). Post-hoc testing, however, did not yield significant results in any of the four areas examined, and there is a good amount of overlap in the confidence intervals in Table 2 and in simultaneous confidence regions for the mean CW and MCS (Figure 4).

Possible influence of the time each brain spent in fixative (Table 1) was tested separately using nonparametric statistics, since the distribution of time in storage is non-normal, as is its logarithm. For the subset of 13 control cases, none of the four dependent variables were significantly correlated with time in storage. The strongest relationship between a dependent variable and time in fixative was observed for CW, with a Spearman rank correlation coefficient $\mathrm{r}=-0.032(\mathrm{p}=0.56)$.

\section{Discussion}

The present study found no differences in the morphometric parameters of minicolumns when considering interactions of hemisphere by diagnosis. This is not surprising as only one area (M1) of the sampled regions exhibits cerebral dominance. A GLI study has shown that the neuropil space is lateralized in human motor cortex (Amunts et al., 1997). Asymmetry in this area may be related to handedness as well as gesture (Hocheberg and LeMay, 1975; Hopkins and Morris, 1989; Hopkins et al., 1990). Our sampling within M1 may have strayed beyond the area controlling handedness thus diluting any findings accrued to cerebral dominance.

The findings with respect to MCS in schizophrenia were not significant on post hoc analysis. Therefore, the findings are considered tentative in nature. One possibility is that interneuronal spacing (mean cell spacing or MCS in our study) is altered in the brains of schizophrenic patients. If a significant reduction of minicolumnar neuropil space had occurred where the majority of cell soma reside, the findings would indicate a defect among the projection systems present in that compartment: apical dendrite clusters and most of the thalamocortical and corticocortical input (Ong and Garey, 1990; Selemon et al., 1995). However, our findings do not indicate changes within the cell core compartment of the minicolumn (e.g., columnar width) but rather within different lamina. The evidence thus suggests a defect within the extrathalamic system that innervates the neocortex in a horizontal or laminar fashion. One implication is that the source of the pathology lies in projections stemming from a source or sources outside of the neocortex. 
Overall, transmission of information within the neocortex occurs within vertical strips of grey matter called minicolumns. In reviewing cortical microcircuits, including the minicolumn, Silberberg et al. (2002) observed great variability accrued to species, region, area, and age. Despite this variability, the existence of stereotypical features indicates a deterministic basis for cortical microcircuits. It may be a general principle of cortical organization that neurons with similar stimulus selection are found in close proximity to each other (Reich et al., 2001). If this is the case, the minicolumn is a possible way of organizing them into a vertical component.

Neurons within the cortex participate in canonical circuits (Douglas and Martin, 2004) that exhibit relative independence from neighboring units (Casanova et al., 2003; Silberberg et al., 2002). Unsurprisingly, the vast majority of connections within the minicolumn are intrinsic to the same, giving rise to massive excitatory and inhibitory connections (Braitenberg and Schüz, 1991; Casanova et al., 2003). Slower-onset modulatory effects are provided by horizontal components that innervate different layers of the minicolumn, e.g., monoaminergic agents that bypass the thalamus. The result provides for a Cartesian-like system for the action of neurotransmitters. This fact may help explain why amino acids (e.g., glutamate, GABA) are the most abundant neurotransmitters in the mammalian nervous system. On the other hand, modulatory transmitters stemming primarily from sources outside of the minicolumn (e.g., acetylcholine, norepinephrine, dopamine, histamine, and 5-hydroxytryptamine) account for transmission at only a small percentage of cortical synaptic sites.

If confirmed, alterations in mean cell spacing reported in the present study may be secondary to the loss of modulatory input to the cell minicolumns. Alterations in neuronal spacing might also imply a correlated alteration in the vertical dimension of the involved lamina. The present study did not measure the width of the lamina. However, in a previous result from our group, using a different patient population, the mean vertical height for our column segments (lamina III, BA 9) was reduced in the brains of schizophrenics patients (304 $\mu \mathrm{m}$ versus $348 \mu \mathrm{m}$ in controls, $\mathrm{p}=0.058$ ) (Buxhoeveden et al., 2000b). This finding compares favorably with the $7 \%$ reduction in cortical thickness reported within the same region reported by Selemon and associates (Selemon et al., 1995).

In our study, variation in MCS as a function of diagnosis was not a uniform feature of the sampled cerebral cortex (see Figures 3 and 4). Studies on the structural pathology of schizophrenia indicate significant but non-uniform reductions in the cortical gray matter. Arguably, changes preferentially affect association cortices such as those located within the superior temporal gyrus and the dorsal prefrontal cortex (McCarley et al., 1999). These abnormalities are present in first-episode patients and prior to clinical onset of the illness thus suggesting that they are a core feature of the pathology of schizophrenia (Lewis and Lieberman, 2000). Consistent with the interpretation that the nonuniform reduction in cortical gray matter reflects alterations in lamination are findings of decreased levels of synaptophysin (a presynaptic terminal protein) (Glantz and Lewis, 1997), lowered density of dendritic spines to which the synapses may be apposed (Garey et al., 1998), and decreased immunoreactivity of tyrosine hydroxylase and dopamine membrane transporter (Akil et al., 1999) in the cerebral cortex of schizophrenic patients. 
Assessing the clinical consequences (e.g., onset of manifestations) of a developmental disturbance in lamination may prove a difficult endeavor. Developmental studies have shown that, even within the same cortical area, individual neurochemical systems may exhibit different patterns of lamination. These patterns depend on the specific cortical layer and type of synapse examined. This finding has led researchers to conclude that, "it is not likely that short periods of environmental disruption will uniformly disturb all neurochemical systems or even one system in all brain areas, but rather individual functional domains may be selectively affected" (Levitt, 2003). Furthermore, postnatal remodeling of the ontogenetic laminar organization is a hallmark of the primate brain. Prominent and relatively complex layer-specific remodeling of the dopaminergic system is still patent in the rhesus monkey at 5 years of age (18 to 20 years in human beings) (Levitt, 2003). This time period is marked by life changes, demands for complex problem solving, and stress. In some patients, primarily males, this time frame coincides with the age of onset of schizophrenia.

Schizophrenia has been linked to prenatal risk factors, particularly to influenza epidemics. Offsprings of mothers exposed to the virus during the second trimester have been shown to have an increased rate of becoming schizophrenic (Limosin et al., 2003). Similarly, the rate of schizophrenia is nearly three times greater in Rhesus (Rh) positive infants born to $\mathrm{Rh}$ negative mothers (Hollister et al., 1996). Rh incompatibility is associated with the creation and transfer of maternal antibodies to the fetus during the second trimester of gestation. This results in hemolytic anemia and brain damage to the fetus during this period of pregnancy. When starvation is examined as a risk factor, it is found that only the offspring of women exposed to famine during their first trimester of pregnancy have an increased rate of hospitalization for schizophrenia (Susser et al., 1996). Whether first or second trimester, epidemiological studies on schizophrenia have offered conflicting results regarding a window of selective vulnerability for the developing brain.

Studies of the prenatal brain have shown that the more primitive parts (e.g., brainstem) develop before more complex and evolved structures (e.g., cerebral cortex). Whenever any brain region is rapidly growing/developing it is most vulnerable to injury. The disparity in timing among risk factors could be the result of a noxious agent's (e.g., starvation) effect on the brainstem during the first trimester. Contrariwise, another agent (c.g., influenza virus) may selectively target brainstem projection to the cerebral cortex during the second trimester. Despite differences in timing, either lesion would provide a disturbance in cortical lamination and connectivity by targeting the brainstem nuclei or their efferents.

One could argue that any lamination disturbance in schizophrenic patients may represent a side effect of neuroleptics. However, cortical cytoarchitectural studies have found no correlations with treatment exposure (Harrison, 1999), and little or no effects of the drugs upon similar parameters have been found in the rat brain (Harrison, 1993). It is therefore reasonable to assume that the results of the present study do not depend on the antipsychotic medication of the patients, but rather indicate neuropathological abnormalities of the illness itself. 
Another possibility to the reported laminar abnormalities relates to alterations in the level of Reelin. Reductions in Reelin mRNA and protein have been shown in frontal, hippocampal and cerebellar cortices of schizophrenic patients by Impagnatiello and associates (Impagnatiello et al., 1995). The results have been reproduced by the same group in additional subjects while another study has shown reduced Reelin immunoreactivity in both schizophrenia and bipolar patients (Fatemi et al., 2000). In the reeler mutant mouse, an absence of Reelin provides for severe alterations that include misroured fibers forming abnormal patches at their terminal zones (Borrell et al., 1999). Some of these abnormalities are transient and not detectable in the adult reeler mouse. These findings have led researchers to postulate that Reelin has a modulatory role during the formation of layers and specific topographic connections (Borrell et al., 1999).

Currently the basis for laminar abnormalitics in schizophrenia, if present, remains unknown. Previous studies that could clarify this putative abnormality have implemented faulty research designs. Thus, reductions in the level of Reelin have been reported following postnatal hypoxia (Curristin et al., 2002). In schizophrenia, the preagonal state of the patients, e.g. suicide by asphyxiation, may therefore offer a confound to significant alteration in Reelin levels. Furthermore, no one during the last century of neuropathological studies in schizophrenia has described the characteristic reversal in the normal pattern of lamination observed in the Reeler mouse cortex. Similarly, older cell counting studies of brainstem nuclei (that provide laminar projections to the cortex) used biased quantitative techniques. More recent studies of cortical lamination in schizophrenia have provided measurements of pseudo-optical density while using techniques that have no relative or absolute quantitative abilities, e.g., immunocytochemistry.

In summary, the data presented in this study indicate a laminar disturbance in the neocortex of schizophrenic patients. Normal measurements in minicolumnar width, variability in minicolumnar width, and verticality index, suggest an abnormality that postdates the development of the cell minicolumn. Our discussion has therefore expounded on a possible alteration of the modulatory systems of the cell minicolumn. This putative disruption of the systems that modulate information processing through the cell minicolumn may account for the range in both severity and symptoms that defines schizophrenia as a clinical syndrome.

\section{Acknowledgements}

The authors wish to thank S. Cleven, J. Mueller and A. Sund for their excellent technical assistance, and M. Schüler and G. Ulmar for assessing the clinical notes of the patients investigated in this study. This article is based upon work supported by the Stanley Medical Research Foundation (H.H., C.S., and M.F.C.), the National Alliance for Autism Research (M.F.C.), and NIMH grants MH61606 (M.F.C.), MH62654 (M.F.C.), and MH69991 (M.F.C). 


\section{References}

Akil, M., Pierri, J.N., Whitehead, R.E., Edgar, C.L., Mohila, C., Sampson, A.R., Lewis, D.A., 1999. Laminaspecific alterations in the dopamine innervation of the prefrontal cortex in schizophrenic subjects. American Journal of Psychiatry 156, 1580-1589

Amunts, K., Schmidt-Passos, F., Schleicher, A., Zilles, K., 1997. Postnatal development of interhemispheric asymmetry in the cytoarchitecture of human area 4. Anatomy and Embryology 196, 393-402

Borrell, V., Del Río, J.A., Alcántara, S., Derer, M., Martínez, A., D'Arcangelo, G., Nakajima, K., Mikoshiba, K., Derer, P., Curran, T., Soriano, E., 1999. Reelin regulates the development and synaptogenesis of the layerspecific entorhino-hippocampal connections. Journal of Neuroscience 19, 1345-1358

Braak, H., Braak, E., 1995. Staging of Alzheimer's disease-related neurofibrillary changes. Neurobiology of Aging 16, 271-278

Braitenberg, V., Schüz, A., 1991. Anatomy of the Cortex: Statistics and Geometry. Springer-Verlag, Heidelberg

Brodmann, K., 1909. Vergleichende Lokalisationslehre der Großhirnrinde. Barth, Leipzig

Buxhoeveden, D.P., Switala, A.E., Roy, E., Casanova, M.F, 2000a. Quantitative analysis of cell columns in the cerebral cortex. Journal of Neuroscience Methods 97, 7-17

Buxhoeveden, D.P., Roy, E., Switala, A.E., Casanova, M.F., 2000b. Reduced interneuronal space in schizophrenia. Biological Psychiatry 47, 681-683

Carpenter M. (1985). Core Text of Neuroanatomy. Third edition. Baltimore: Williams and Wilkins

Casanova, M.F., Buxhoeveden, D.P., Gomez, J., 2003. Disruption in the inhibitory architecture of the cell minicolumn: Implications for autism. The Neuroscientist 9, 496-507

Casanova, M.F., Switala, A.E., 2005. Minicolumnar morphometry: computerized image analysis. In: Casanova, M.F. (Ed.), Neocortical Modularity and the Cell Minicolumn. Nova Science Publishers, New York, in press

Curristin, S.M., Cao, A., Stewart, W.B., Zhang, H., Madri, J.A., Morrow, J.S., Ment, L.R., 2002. Disrupted synaptic development in the hypoxic newborn brain. Proceedings National Academy of Sciences of the United States of America 99, 16729-16734

Davison, A.C., Hinkley, D.V., 1997. Bootstrap methods and their application. Cambridge University Press, Cambridge

Douglas, R.J., Martin, K.A.C., 2004. Neocortical circuits of the neocortex. Annual Review of Neuroscience 27, $419-451$

Economo, C. von, Koskinas, G.N., 1925. Die Cytoarchitekonik der Hirnrinde des erwachsenen Menschen. Julius Springer, Wien

Fatemi, S.H., Earle, J.A., McMenomy, T., 2000. Reduction in reelin immunoreactivity in hippocampus of subjects with schizophrenia, bipolar disorder and major depression. Molecular Psychiatry 5, 654-663

Garey, L.J., Ong, W.Y., Patel, T.S., Kanani, M., Davis, A., Mortimer, A.M., Barnes, T.R.E., Hirsch, S.R., 1998. Reduced dendritic spine density on cerebral cortical pyramidal neurons in schizophrenia. Journal of Neurology, Neurosurgery, and Psychiatry 65, 446-453

Glantz, L.A., Lewis, D.A., 1997. Reduction of synaptophysin immunoreactivity in the prefrontal cortex of subjects with schizophrenia: Regional and diagnostic specificity. Archives of General Psychiatry 54, 943-952

Harrison, P.J., 1993. Effect of neuroleptics on neuronal and synaptic structure. In: Barnes, T.R.E. (Ed.), Antipsychotic Drugs and Their Side-Effects. Academic Press, London, pp. 99-110 
Harrison, P.J., 1999. The neuropathology of schizophrenia: A critical review of the data and their interpretation. Brain 122, 593-624

Heinsen, H., Heinsen, Y.L., 1991. Serial thick, frozen, gallocyanin stained sections of human central nervous system. Journal of Histotechnology 14, 167-173

Heinsen, H., Rüb, U., Bauer, M., Ulmar, G., Bethke, B., Schüler, M., Böcker, F., Eisenmenger, W., Götz, M., Korr, H., Schmitz, C., 1999. Nerve cell loss in the thalamic mediodorsal nucleus in Huntington's disease. Acta Neuropathologica 97, 613-622

Heinsen, H., Arzberger, T., Schmitz, C., 2000. Celloidin mounting (embedding without infiltration): A new, simple and reliable method for producing serial sections of high section thickness through complete human brains and its application to stereological and immunohistochemical investigations. Journal of Chemical Neuroanatomy 20, 49-59

Hocheberg, F.H., Le May, M., 1975. Arteriographic correlates of handedness. Neurology 25, 218-222

Hollister, J.M., Laing, P., Mednick, S.A., 1996. Rhesus incompatibility as a risk factor for schizophrenia in male adults. Archives of General Psychiatry 53, 19-24

Hopkins, W.D., Morris, R.D., 1989. Laterality for visual-spatial processing in two language-trained chimpanzees (Pan troglodytes). Behavioral Neuroscience 103, 227-234

Hopkins, W.D., Washburn, D.A., Rumbaugh, D.M., 1990. Processing of form stimuli presented unilaterally in humans, chimpanzees (Pan troglodytes), and monkeys (Macaca mulatta). Behavioral Neuroscience 104, 577-582

Impagnatiello, F., Guidotti, A.R., Pesold, C., Dwivedi, Y., Caruncho, H., Pisu, M.G., Uzunov, D.P., Smalheiser, N.R., Davis, J.M., Pandey, G.N., Pappas, G.D., Tueting, P., Sharma, R.P., Costa, E., 1995. A decrease of reelin expression as a putative vulnerability factor in schizophrenia. Proceedings of the National Academy of Sciences of the United States of America 95, 15718-15723

Levitt, P., 2003. Structural and functional maturation of the developing primate brain. Journal of Pediatrics 143, s35-s45

Lewis, D.A., Lieberman, J.A., 2000. Catching up on schizophrenia: Natural history and neurobiology. Neuron $28,325-334$

Limosin, F., Rouillon, F., Payan, C., Cohen J.M., Strub N., 2003. Prenatal exposure to influenza as a risk factor for adult schizophrenia. Acta Psychiatrica Scandinavica 107, 331-335

McCarley, R.W., Wible, C.G., Frumin, M., Hirayasu, Y., Levitt, J.J., Fischer, I.A., Shenton, M.E., 1999. MRI anatomy of schizophrenia. Biological Psychiatry 45, 1099-1119

Mountcastle, V.B.1998. Perceptual Neuroscience: the Cerebral Cortex. Cambridge (MA): Harvard University Press

Ong, W.Y., Garey, L.J., 1990. Neuronal architecture of the human temporal cortex. Anatomy and Embryology $181,351-369$

Otsu, N., 1979. A threshold selection method from grey-level histograms. IEEE Transactions on Systems, Man, and Cybernetics 9, 377-393

Pourdeyhimi, B., Xu, B., Nayernouri, A., 1994. Evaluating carpet appearance loss: Pile lay orientation. Textile Research Journal 64, 130-135

Rajkowska, G., Goldman-Rakic, P.S., 1995. Cytoarchitectonic definition of prefrontal areas in the normal human cortex, I: remapping of areas 9 and 46 using quantitative criteria. Cerebral Cortex 5, 307-322

Rajkowska, G., Goldman-Rakic, P.S., 1995b. Cytoarchitectonic definition of prefrontal areas in the normal human cortex, II: variability in locations of areas 9 and 46 and relationship to the Talairach Coordinate System. Cerebral Cortex 5, 323-337 
Chapter 6

Reich, D.S., Mechler, F., Victor, J.D., 2001. Independent and redundant information in nearby cortical neurons. Science 294, 2566-2568

Selemon, L.D., Rajkowska, G., Goldman-Rakic, P.S., 1995. Abnormally high neuronal density in the schizophrenic cortex: A morphomerric analysis of prefrontal area 9 and occipital area 17. Archives of General Psychiatry 52, 805-820

Silberberg, G., Gupta, A., Markram, H., 2002. Stereotypy in neocortical microcircuits. Trends in Neurosciences $25,227-230$

Susser, E., Neugebauer, R., Hoek, H., Brown, A.S., Lin, S., Labovitz, D., Gorman J.M., 1996. Schizophrenia after prenatal famine: Further evidence. Archives of General Psychiatry 53, 25-31

Vogeley, K., Tepest, R., Schneider-Axmann, T., Hütte, H., Zilles, K., Honer, W.G., Falkai, P., 2003. Automated image analysis of disturbed cytoarchitecture in Brodmann area 10 in schizophrenia. Schizophrenia Research $62,133-140$

Vogt, C., Vogt, O., 1919. Allgemeinere Ergebnisse unserer Hirnforschung. Dritte Mitteilung. Die architektonische Rindenfelderung im Lichte unserer neuesten Forschungen. Journal für Psychologie und Neurologie $25,361-376$ 


\section{Tables}

Table 1. Clinical characteristics of the cases included in this study. PMI: interval between death and autopsy. Storage: duration of immersion in $10 \%$ formalin. All schizophrenic patients and all controls were male.

\begin{tabular}{|c|c|c|c|c|c|c|}
\hline \multirow{2}{*}{ No. } & \multirow{2}{*}{$\begin{array}{c}\text { Age } \\
\text { [years] }\end{array}$} & \multirow{2}{*}{ Cause of death } & \multirow{2}{*}{ PMI [hours] } & \multirow{2}{*}{$\begin{array}{c}\text { Storage } \\
\text { [days] }\end{array}$} & \multicolumn{2}{|c|}{ Diagnosis } \\
\hline & & & & & DSM-IV & ICD-10 \\
\hline S1 & 22 & Suicide & $88^{1}$ & 130 & 295.30 & $F 20.0^{2}$ \\
\hline $\mathrm{S} 2$ & 36 & Suicide & $<72^{1}$ & 115 & 295.30 & $F 20.0^{2}$ \\
\hline $\mathrm{S} 3$ & 46 & Systemic hypothermia & $<24$ & 327 & 295.30 & $F 20.01^{2}$ \\
\hline$\$ 4$ & 50 & Peritonitis & $<24$ & 203 & 295.30 & $F 20.0^{2}$ \\
\hline $\mathrm{S} 5$ & 50 & Suicide & 18 & 170 & 295.30 & $F 20.0^{2}$ \\
\hline 56 & 51 & Septicemia & 33 & 127 & 295.60 & $F 20.50^{3}$ \\
\hline 57 & 54 & Septicemia & 27 & 250 & 295.60 & $F 20.50^{3}$ \\
\hline $\mathrm{S} 8$ & 55 & Right-sided heart failure & 25 & 84 & 295.30 & $F 20.00^{2}$ \\
\hline$\$ 9$ & 57 & Septicemia & 76 & 163 & 295.30 & $F 20.00^{2}$ \\
\hline 510 & 60 & Pulmonary embolism & $<48$ & 311 & 295.30 & $F 20.01^{2}$ \\
\hline$\$ 11$ & 62 & Aspiration & 7 & 171 & 295.30 & $\mathrm{~F} 20,00^{2}$ \\
\hline$\$ 12$ & 63 & Acute myocardial infarct & 15 & 338 & 295.60 & $F 20.50^{3}$ \\
\hline$\$ 13$ & 64 & Pulmonary embolism & 6 & 817 & 295.60 & $F 20.50^{3}$ \\
\hline $\mathrm{Cl}$ & 25 & Cardiac tamponade & 14 & 119 & & \\
\hline $\mathrm{C} 2$ & 36 & Gunshot & 24 & 143 & & \\
\hline $\mathrm{C3}$ & 47 & Acute myocardial infarct & $<24$ & 133 & & \\
\hline $\mathrm{C4}$ & 50 & Acute myocardial infarct & 35 & 433 & & \\
\hline$C 5$ & 50 & Avalanche accident & 23 & 498 & & \\
\hline $\mathrm{C} 6$ & 51 & Septicemia & 7 & 285 & & \\
\hline $\mathrm{C} 7$ & 54 & Acute myocardial infarct & 18 & 168 & & \\
\hline $\mathrm{C} 8$ & 56 & Acute myocardial infarct & 60 & 3570 & & \\
\hline $\mathrm{Cg}$ & 58 & Acute myocardial infarct & 28 & 126 & & \\
\hline $\mathrm{C} 10$ & 60 & Gastrointestinal hemorrhage & 18 & 101 & & \\
\hline $\mathrm{C} 11$ & 60 & Gastrointestinal hemorrhage & 27 & 302 & & \\
\hline $\mathrm{Cl} 2$ & 62 & Acute myocardial infarct & $<24$ & 3696 & & \\
\hline$C 13$ & 65 & Bronchopneumonia & 6 & 2289 & & \\
\hline
\end{tabular}

These patients had long post mortem intervals (PMI). However, both patients committed suicide (one by hanging, the other by jumping from an eight story building), were found within $1 \mathrm{~h}$ of death, and were kept at $4^{\circ} \mathrm{C}$ until autopsy. Accordingly, the intervals between death and autopsy of these patients cannot be compared to the corresponding intervals of the other persons and were thus excluded from the calculation of the mean PMI between death and autopsy.

2Paranoid schizophrenia.

${ }^{3}$ Residual schizophrenia.

PMl: interval between death and autopsy. Storage: duration of immersion in 10\% formalin. All schizophrenic patients and all controls were male. 
Table 2. An example of resampling with replacement. This simulated data set includes the measurements from patient $\mathrm{S1}$, control $\mathrm{C} 1$, three replicates of patient $\mathrm{S} 3$, and so forth. It is possible to generate $1326 \approx 1029$ distinct data sets in this fashion.

\begin{tabular}{ccccccccccccccc} 
Group & 1 & 2 & 3 & 4 & 5 & 6 & 7 & 8 & 9 & 10 & 11 & 12 & 13 & Total \\
\hline Schizophrenic & 1 & & 3 & 1 & & 2 & 2 & 1 & & 1 & & 1 & 1 & 13 \\
Control & 1 & & 2 & 1 & 1 & 1 & 1 & & 3 & 1 & 2 & & & 13 \\
\hline
\end{tabular}

Table 3. Estimated mean, standard deviation, and $95 \%$ confidence intervals for mean interneuronal distance (MCS), by diagnosis and cortical area, averaged across laminas III and V. Means and standard deviations are also provided for the other three parameters (CW, VCW, $\mathrm{Cl})$, which exhibited no significant diagnosis dependence or diagnosis interaction.

\begin{tabular}{|c|c|c|c|c|c|c|c|c|c|c|}
\hline \multirow{2}{*}{$\begin{array}{c}\text { Cortical } \\
\text { Area }\end{array}$} & \multirow[b]{2}{*}{ Group } & \multicolumn{3}{|c|}{$\operatorname{MCS}(\mu \mathrm{m})$} & \multicolumn{2}{|c|}{$C W(\mu \mathrm{m})$} & \multicolumn{2}{|c|}{$\operatorname{VCW}(\mu \mathrm{m})$} & \multicolumn{2}{|c|}{$\mathrm{Cl}$} \\
\hline & & Mean & S.D. & Interval & Mean & S.D. & Mean & S.D. & Mean & S.D. \\
\hline & $S$ & 21.8 & 4.2 & {$[21.3,22.3]$} & 54.7 & 7.8 & 19.4 & 4.1 & 0.346 & 0.168 \\
\hline BA9 & C & 21.2 & 4.4 & {$[20.7,21.7]$} & 53.8 & 8.2 & 19.5 & 4.3 & 0.331 & 0.176 \\
\hline & S & 22.4 & 4.8 & {$[21.9,23.0]$} & 55.6 & 8.8 & 19.8 & 4.6 & 0.346 & 0.190 \\
\hline M1 & C & 22.0 & 4.2 & {$[21.5,22.5]$} & 56.0 & 7.8 & 20.3 & 4.1 & 0.349 & 0.167 \\
\hline & S & 19.5 & 5.5 & {$[18.9,20.2]$} & 52.8 & 10.3 & 19.4 & 5.4 & 0.358 & 0.222 \\
\hline 51 & $C$ & 20.1 & 4.2 & {$[19.7,20.6]$} & 53.6 & 7.8 & 19.8 & 4.1 & 0.355 & 0.168 \\
\hline & $\mathrm{S}$ & 15.6 & 5.0 & {$[15.1,16.2]$} & 43.2 & 9.2 & 17.0 & 4.8 & 0.242 & 0.198 \\
\hline $\mathrm{V} 1$ & C & 16.4 & 4.3 & {$[15.9,16.9]$} & 44.3 & 8.0 & 16.7 & 4.2 & 0.261 & 0.171 \\
\hline
\end{tabular}

Mean: estimated population mean; S.D.: estimated population standard deviation; S: schizophrenic patients; C: controls; BA9: Brodmann's area 9; M1, S1, and V1: primary motor, sensory, and visual cortical areas, respectively. 


\section{Figures}

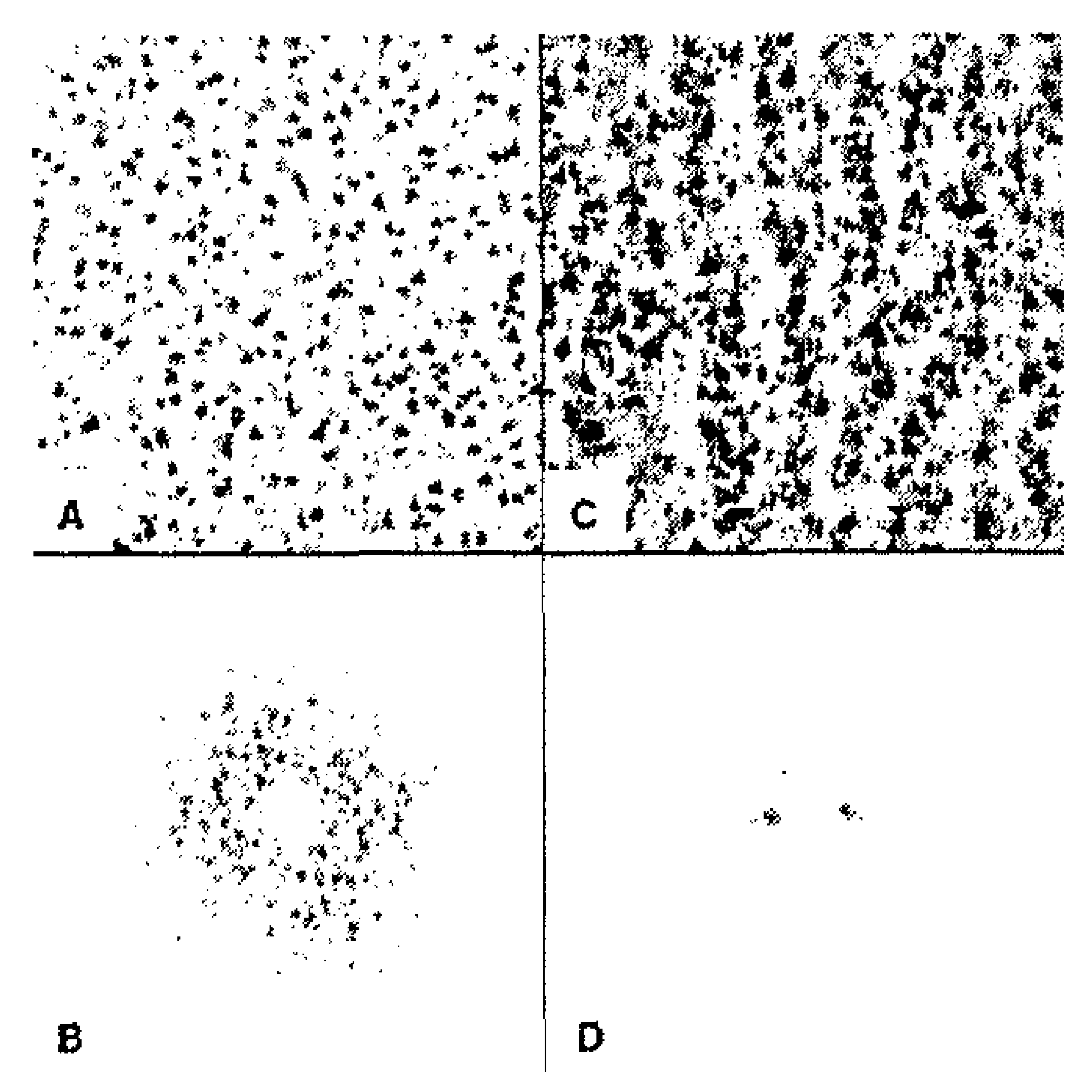

Fig. 1. An image of a seemingly isotropic patch of cortex (A) has its power, estimated with a Fast Fourier Transform (FFT), distributed evenly about the origin (B). By contrast, an image of a region of cortex showing clear columnar structure $(C)$ has its power distribution (estimated with a FFT) concentrated into two lobes symmetric about the origin (D). This is the rationale for the columnarity index $\mathrm{Cl}$ : the degree to which the image spectrum is concentrated along one axis. The origin of Fourier space is the center of images $B$ and $D$.

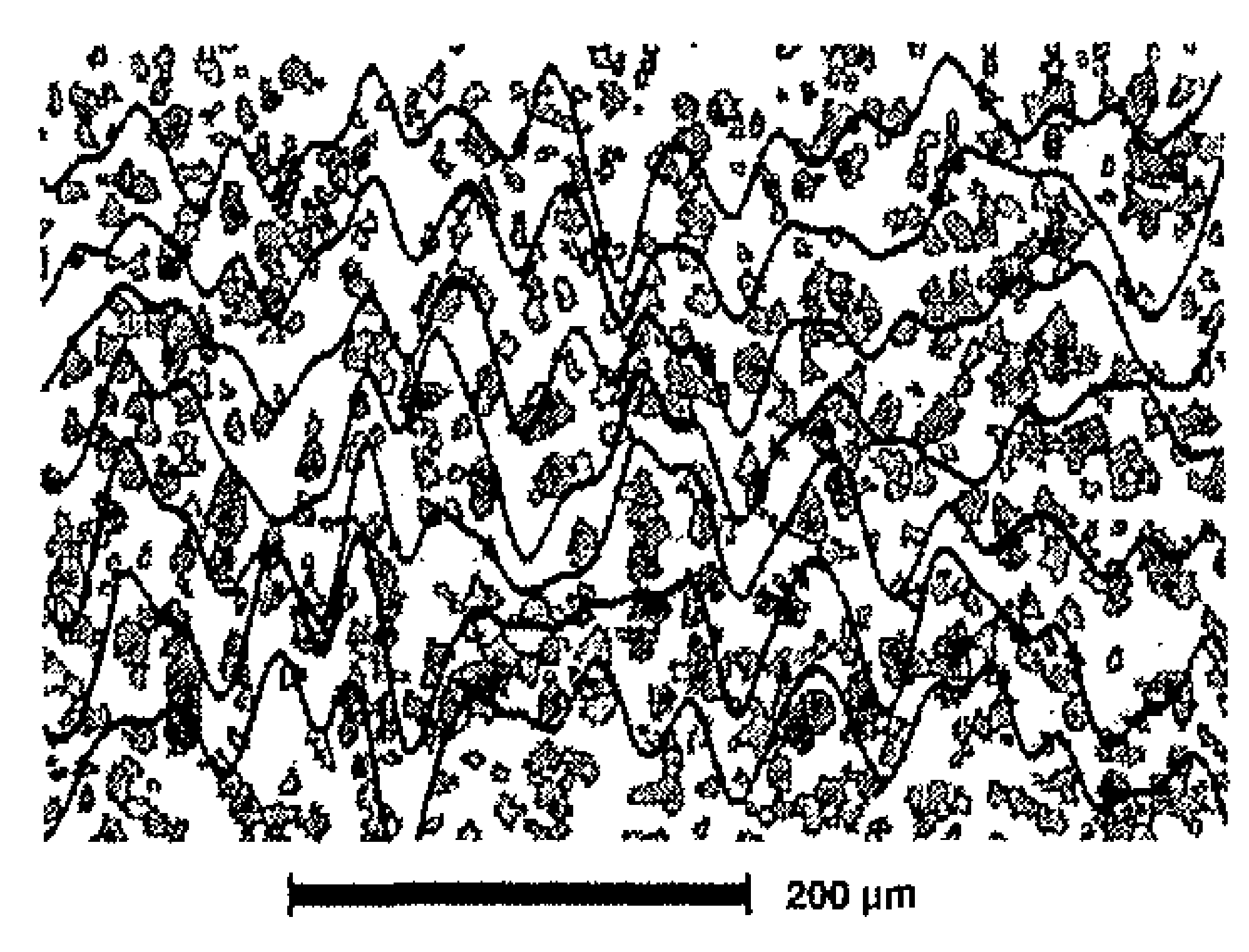

Fig. 2. Superposed on an image of cortical layer III are the boundaries of cells (dark outlines) as determined by the automatic segmentation algorithm, and the resulting cell density profiles at several depths (smooth curves), which determine the estimate of minicolumnar width (CW). 


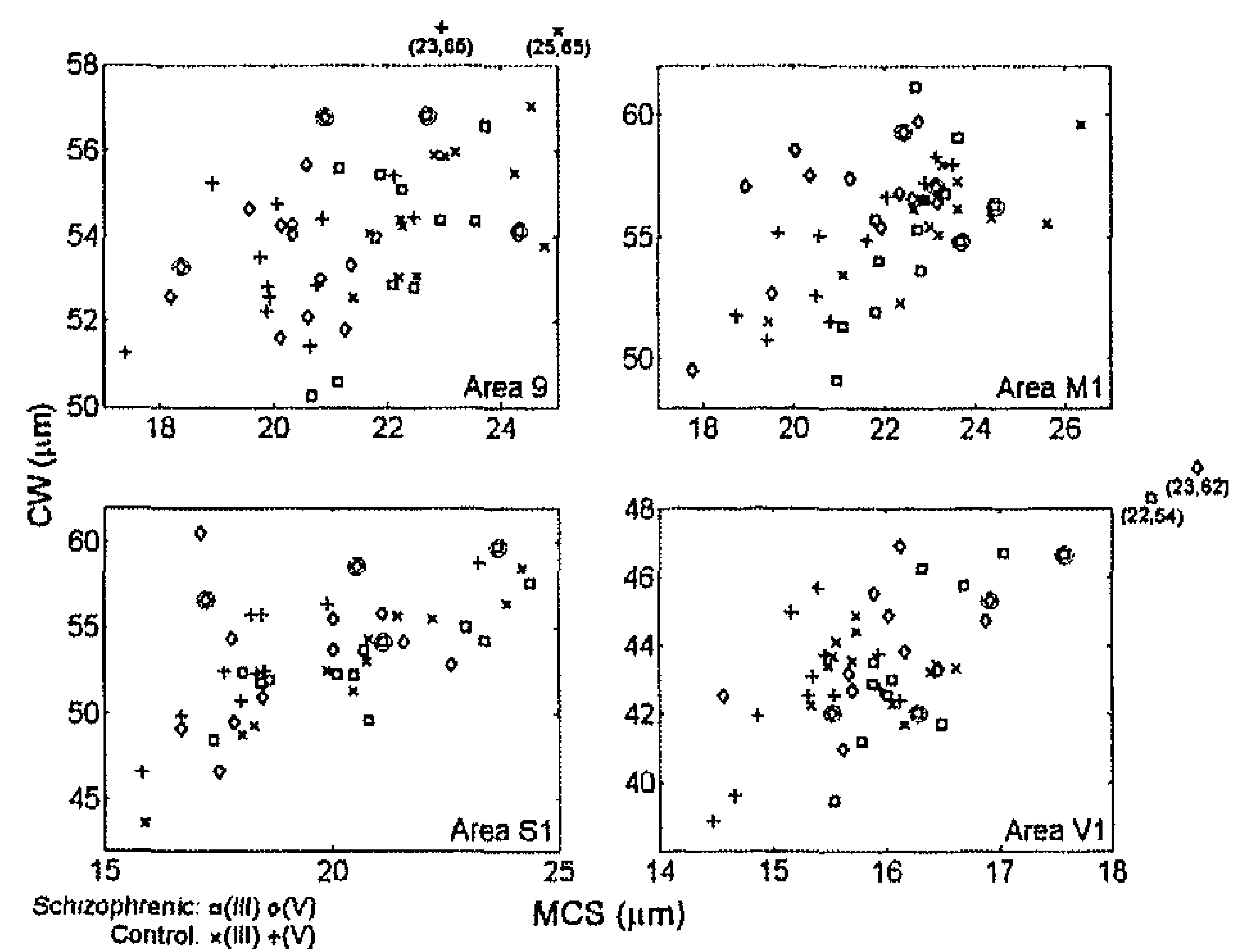

Fig. 3. Raw measurements of minicolumnar width and interneuronal distance, broken down by cortical area, lamina, and diagnostic group. Observations from the two schizophrenic patients with long post-mortem intervals (S1 and S2 in table 1) are circled. Measurements are given in parentheses for mild outliers in areas 9 and 17, where the axis limits were narrowed to better show the scatter in the bulk of the data.

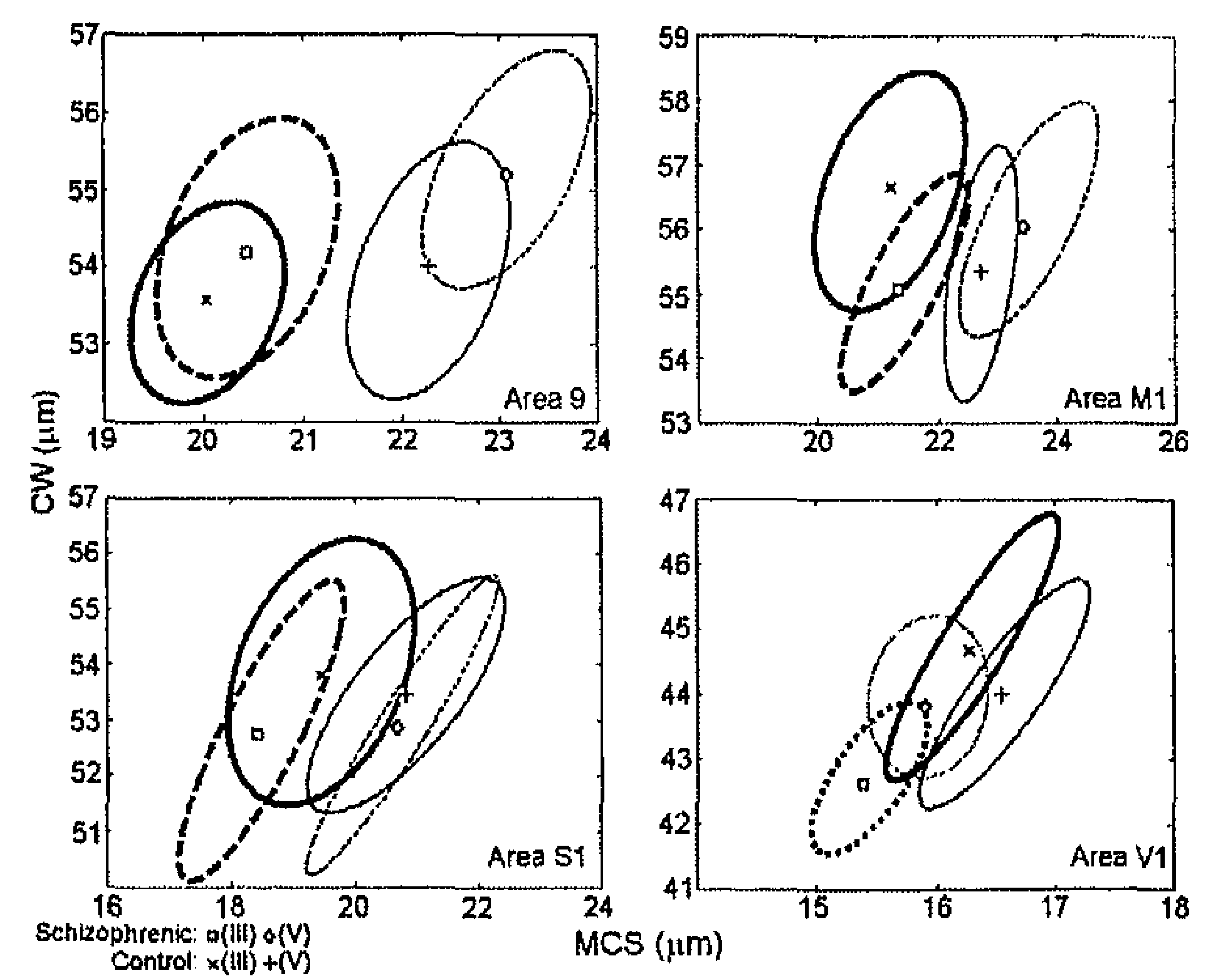

Fig. 4. Bootstrap $95 \%$ confidence ellipses, by cortical area, for the mean minicolumn width (CW) and interneuronal distance (MCS). Also shown are the maximum likelihood estimates for the means (points). Solid lines: control. Broken lines: schizophrenia. Thin lines: lamina III. Bold lines: lamina $\mathrm{V}$. 
Chaprer 7

General discussion and future implications 
Postmortem studies in the literature of brains of patients with schizophrenia revealed subtle neuropathological abnormalities in several cortical and subcortical regions. However, many of the reported data were divergent if not mutually contradictory. No clear pattern of morphological alterations has yet been established. The problem, according to some researchers (Harrison, 1999) lies mainly within methodological differences, investigated parameters and particularly in the availability of brain samples from patients with schizophrenia and matched controls.

By means of this thesis I am presenting a solution to this situation:

- simultaneous analysis of several cortical and subcortical regions of the same brains;

- application of state-of-the-art quantitative design-based stereologic techniques; and

- analysis of a sample of well documented postmortem brains from 13 male patients with schizophrenia and 13 age-matched male controls.

In Chapter 2 I demonstrated a distinct pattern of subtle neuropathological alterations in several subcortical regions. This previously unrecognized set of alterations comprises of:

- reduced volumes of the total cortical gray matter, putamen and nucleus accumbens;

- reduced total neuron numbers in the caudate nucleus, putamen and the lateral nucleus of the amygdala; and

- no correlation between the illness duration of the patients with schizophrenia and the observed neuropathological alterations.

The results from the control cases were in line with previous studies. The results obtained for the patients with schizophrenia can be compared to data in the literature as follows.

\section{Cortical gray matter}

A significant decrease in the total cortical gray matter volume (-9.3\%; averaged for the left and right hemispheres) was found when comparing the patients with schizophrenia to the controls. This result was in agreement with several reports in the literature (Zipurski et al., 1992; 1997; McCarley et al., 1999; Shenton et al., 2001)

\section{Caudate nucleus, putamen and nucleus accumbens}

Several clinical neuroimaging studies which have shown an enlargement of the striatum are in contrast to a postmortem study of Beckmann and Lauer (1997), who reported no differences in mean volumes of the caudate nucleus and the putamen, as well as Bogerts (1984) who found a trend towards reduced mean volumes of the putamen in schizophrenia. On the other hand, several neuroimaging studies reported a decrease in the mean volume of the striatum in drug-naive patients with schizophrenia and a reduction of the mean volume in basal ganglia of patients with schizophrenia after switching to olanzapine (Harrison, 1999; Wright et al., 2000; Shenton et al., 2001). An increase of the mean volume of the striatum in schizophrenia in those studies could have been caused by an yet unknown, but reversible action of typical neuroleptics or during histological processing.

The discrepancies of the mean total neuron numbers in the caudate nucleus and the putamen between the present study and the analysis by Beckmann and Lauer (1997) might 
be related to differences in the stereological design of the studies and the analyzed samples of postmortem brains. Anyway, the number of unbiased virtual counting spaces used to count neurons by Beckmann and Lauer (1997) was considerably lower than in the present study, which implies a considerably higher variation in estimated total neuron numbers Schmitz, 1998; Schmitz and Hof, 2000; 2005).

Pakkenberg's (1990) early report of a reduced mean volume of the nucleus accumbens $(-42 \%)$ and a reduced mean total neuron number $(-50 \%)$ in postmortem brains of patients with schizophrenia should be taken cautiously as already discussed by Lauer and Beckmann (1997). Bogerts (1984) found almost no differences in the mean volumes of the nucleus accumbens between patients with schizophrenia and controls. The discrepancy between the results of Pakkenberg (1990), Bogerts (1984), Lauer and Beckmann (1997) and the present study might be due to differences in the stereological design. For instance, Pakkenberg (1990) performed microscopic investigations on $4 \mu \mathrm{m}$-thick paraffin sections. Delineations of the brain regions on such sections might be much more difficult than on sections of $20 \mu \mathrm{m}$ (Bogerts, 1984), $500 \mu \mathrm{m}$ (Lauer and Beckmann, 1997) or 600-700 $\mu \mathrm{m}$ (present study).

\section{Mediodorsal nucleus of the thalamus}

The reduction of the mean volume of the mediodorsal nucleus of the thalamus in schizophrenia (as reported in several studies; Pakkenberg, 1990; Popken et al., 2000; Byne et al., 2002; these studies with positive outcome are henceforth referred to as PO studies here) could not be confirmed in the present study as well as in other studies in the literature (Cullen et al., 2003; Kemether et al., 2003; Dorph-Petersen et al., 2004; Danos et al., 2005; these studies with negative outcome are henceforth referred to as NO studies here). Again, differences in the study design may explain this discrepancy. The PO studies had on average smaller sample sizes than the NO studies. Furthermore, except of the study by Dorph-Petersen et al. (2004) in all NO studies both the left and right mediodorsal nucleus of the thalamus were investigated, and the mean age of the patients was higher in the PO studies than in the NO studies. In this regard small sample sizes and confounding pathologies (possible age-related neurodegeneration) cannot be excluded as a major potential obstacle in most neuropathological studies on schizophrenia (Harrison, 1999).

Amygdala

Meta-analyses of clinical neuroimaging studies reported reductions in the mean volume of the amygdala in patients with schizophrenia in the range of 5 to $10 \%$ (Lawrie and Abukmeil, 1998; Nelson et al., 1998; Wright et al., 2000; see also Honea et al., 2005). However, no differentiation between the different nuclei of the amygdala were performed in these studies. These data are in line with the results of the present study as well as of a postmortem study by Bogerts (1984). In contrast, no mean volume reduction in schizophrenia was found by Heckers et al. (1990) and Chance et al. (2002). Nevertheless, according to the latter authors alterations of other morphological aspects such as cellular composition of the amygdala cannot be excluded. 


\section{Asymmetry of brain regions}

Although suggested by several reports in the literature (Crow, 1990; 1997; Patty, 1999) no significant differences between the right and left hemispheres were found in the present study. A meta-analysis of clinical neuroimaging studies, in which a volume of $100 \%$ was assumed for the control group, found an overall mean volume of $94 \%$ for both the left and right amygdala in patients with schizophrenia. Disturbance of cerebral asymmetry in schizophrenia might therefore be restricted to cortical regions such as the dorsolateral prefrontal cortex (Cullen et al., 2006)

In Chaprers 3 and $4 \mathrm{I}$ assessed the integrity of the microvasculature in several subcortical and cortical brain regions in schizophrenia. To this end I investigated variables such as microvessel length density, total microvessel length and microvessel length per neuron in the caudate nucleus, putamen, nucleus accumbens, mediodorsal nucleus of the thalamus, and lateral nucleus of the amygdala. The patients with schizophrenia showed a statistically significant increase in mean microvessel length density in the caudate nucleus and in the mediodorsal nucleus of the thalamus, and a decrease in mean total microvessel length in the lateral nucleus of the amygdala. The stereological data presented in Chapter 2 allowed then to asscss possible changes in total microvessel length and mean microvessel length per neuron in these regions. There were, however, no significant differences between the patients with schizophrenia and the controls with respect to the mean microvessel length per neuron in all investigated subcortical areas. Furthermore, the analysis of the mean capillary length density within cortical areas 9 and 24 also showed no significant differences between patients with schizophrenia and controls. Only the combination of diagnosis and hemisphere had a significant effect on the mean capillary length density within both areas 9 and 24 .

The postmortem interval can affect stereologic estimates of cerebral blood vessels (Ingvar and Franzen, 1974). In the present study the postmortem interval had a significant effect on the total microvessel length in the putamen and the mediodorsal nucleus of the thalamus. However, post-hoc linear regression analysis revealed no significant correlations between the postmortem interval (or the fixation time) and any other investigated variable.

In Chapter 5 I demonstrated that mean cell spacing abnormalities in the cerebral cortex of parients with schizophrenia were not significant in post-hoc analyses and therefore tentative in nature. It is however possible that the interneuronal mean cell spacing is altered in the brains of patients with schizophrenia. The findings indicate changes rather within different lamina than within the cell core compartment of minicolumns. Moreover, the alterations in mean cell spacing may be secondary to the loss of modulatory input to the cell minicolumns. Therefore, these alterations do not indicate a single mechanism that can be found in all regions but rather a distuption of the systems that modulate information processing, another aspect of the wiring, cytoarchitecture and cell morphology alterations of the brain of patients with schizophrenia. Direct conclusions from the alterations observed cannot be therefore drawn. 
In Chapter 6 I present results of analyses of the neuronal distribution in the neocortex of patients with schizophrenia that are consistent with altered neocortical neuropil reports, a phenomenon well established in the literature (Selemon 1998, Broadbelt 2000 and Kalus 2000). These alterations reflect de facto modulatory systems abnormalities innervating the cell minicolumns.

What is the specific contribution of this thesis to a better understanding of schizophrenia? In general, two non-exclusive hypotheses have been proposed, i.e., the neurodevelopmental hypothesis of schizophrenia (Lieberman, 1999; Tsuang, 2000; Lewis and Levitt, 2002; Murray et al., 2004) and the neurodegenerative hypothesis of schizophrenia (Ashe et al., 2001; Church et al., 2002; Sawa and Snyder, 2002). In recent decades the neurodevelopmental hypothesis of schizophrenia has prevailed (Weinberger, 1987; Lewis and Lewitt, 2002; Murray et al., 2004). In this hypothesis genetic and non-genetic mechanisms are thought to interact, in yet not fully understood ways, affecting the developing brain and resulting in a predisposition to schizophrenia (Schmidt-Kastner et al., 2006). Yet the several genes proposed as candidates (for review see Davis et al., 2003; Harrison and Owen, 2003; Owen et al., 2004; Harrison and Weinberger, 2005; Kirov et al., 2005) may not account for all aspects of the pathogenesis (Kendler et al., 2005). There is no direct evidence for a neurodevelopmental or a neurodegenerative origin of the results presented in this thesis. Yet, reductions in mean volumes (cortical gray matter, putamen and lateral nucleus of the amygdala) and mean total neuron numbers (caudate nucleus, putamen and lateral nucleus of the amygdala) in the brains of patients with schizophrenia did not correlate with the illness duration. Such lack of correlation might be indicative for a neurodevelopmental deficit, particularly with respect to the alterations found in the lateral nucleus of the amygdala. Moreover, lesions of the amygdala in rats obtained during the early postnatal period (but not during adulthood) lead to a combination of behavioral changes with shared features with schizophrenia (Wolterink et al., 2001). Furthermore, the minicolumn analysis indicated the possibility of laminar disturbances in cortical regions and therefore an alteration of the modulatory systems in the brains of patients with schizophrenia. Together with the suggested alterations in the neuropil (mostly by means of laminar thinning rather than decreased neuropil width) the minicolumns analysis supported the neurodevelompental hypothesis and was in line with the stereologically obtained data since those alterations also do not correlate with the illness duration. Furthermore, connections between the basolateral amygdala and the prefrontal cortex develop slowly during postnatal development in rats (Bouwmeester et al., 2002; Cunningham et al., 2002). Postnatal lesions of mesial temporal limbic structures (including the amygdala) in monkeys is associated with abnormal function of the prefrontal cortex of adult animals (Bertolino et al., 1997). The prolonged maturation of connections between the amygdala and prefrontal cortex has been hypothesized to be involved during onset of schizophrenia in adolescence (Bencs, 2003). Reduction of mean total neuron numbers found in the present study in the amygdala can also play a pivotal role in these processes and requires further investigation. 
The small decrease in mean total neuron numbers found in the present study could be due to a prolonged neurodevelopmental perturbation affecting several processes in the striatum, including neurogenesis and the innervation by dopmainergic mesencephalic fibers. Furthermore, the reduced mean total neuron number in the lateral nucleus of the amygdala in schizophrenia could also result in impaired function of the basal nucleus of the amygdala with potential influence on (at least) two functional systems, i.e., the striatal and cortical output of the basal nucleus of the amygdala. This in turn could impair the control of the specific involuntary components of emotional reactions which are impaired in schizophrenia (Aleman and Khan, 2005). Finally, the reduced mean total neuron number in the lateral nucleus of the amygdala in schizophrenia can indirectly (via ventral striatum projections) influence dopaminergic neurons through amygdalo-striato-nigral loops (Haber and Fudge,1997, Fudge et al. 2002).

Analysis of the integrity of the microvasculature in schizophrenia revealed no changes in the mean microvessel length in cortical brain areas 9 and 24 and in the mean microvessel length per neuron in all investigated subcortical brain areas. These data do not support the hypothesis of (structural) alterations in the microvasculature in schizophrenia. Furthermore, these studies (presented in Chapters 3 and 4) were performed in the areas that represent so called "hot spots" for structural abnormalities in schizophrenia. The present negative outcome indicates that the anatomical substrate for abnormal regional cortical blood flow (rCBF) and cerebral blood volume (Brockhaus 1942; Hirai and Jones, 1989; Arnold and Trojanowski, 1996; Middleton et al., 2002; Cullen et al., 2003, ) might be absent. Changes in $\mathrm{rCBF}$ activation are most likely due to region-specific neuronal or glial factors. Alterations in gene and protein expression found in cortical samples from patients with schizophrenia probably reflect an abnormal metabolic state (Schmidt-Kastner et al., 2006), caused by the primary disease process, secondary long-term neuronal dysfunction, chronic medication, or imbalances in agonal events influencing microarray measurements (Weinberger 1987; Jones, 1997; Malaspina et al., 2004; Vawter et al., 2006).

A side observation was that the neuron density was not correlated with the microvessels length density within the five investigated subcortical regions both in the brains from patients with schizophrenia and the controls. This can be explained by the fact that the microvessel length density is linked to metabolic expenditures in neuronal and gial processes whereby neuron density (measured in the present study) or overall cell density do not tightly correlate with the total amount of the functional processes in each structure. Nevertheless the possibility of vascular abnormalities in untreated patients with schizophrenia cannot be excluded. Dopaminergic processes make close contacts with capillaries in the prefrontal cortex and dopamine reduces local perfusion (Lauer and Heinsen, 1996). Thus it is tempting to speculate that abnormal dopamine levels in schizophrenia prior to administration of neuroleptics (which are responsible for chronic dopamine receptor blockade) may have functional and structural effects on the microvasculature before the onset of clinical symptoms.

Finally it should be mentioned that the present observations in schizophrenia must not be misinterpreted as evidence against a possible interaction of hypoxia, the microvascula- 
ture and neurons within the developing brain. Rather neurodevelopmental perturbations that are thought to underlie schizophrenia could in fact be manifested by the occurrence of alterations in microvessel length and microvessel length per neuron in the fetal brain of people who will later develop schizophrenia. However, those alterations might not necessarily persist in the postnatal life in the brains of patients with schizophrenia.

\section{References}

Aleman A, Kahn RS (2005) Strange feelings: do amygdala abnormalities dysregulate the emotional brain in schizophrenia? Prog Neurobiol 77: 283-98

Arnold SE, Trojanowski JQ (1996) Recent advances in defining the neuropathology of schizophrenia. Acta Neuropathol 92:217-231

Ashe PC, Berry MD, Boulton AA (2001) Schizophrenia, a neurodegenerative disorder with neurodevelopmental antecedents. Prog Neuropsychopharmacol Biol Psychiatry 25: 691-707

Andreasen NC, Rezai K, Alliger R, Swayze VW, Flaum M, Kirchner P, Cohen G, O'Leary DS(1992) Hypofrontality in neuroleptic-naive patients and in patients with chronic schizophrenia. Assessment with xenon 133 single-photon emission computed tomography and the Tower of London. Arch Gen Psychiatry 49:943-958

Beckmann H, Lauer M (1997) The human striatum in schizophrenia. II.Increased number of striatal neurons in schizophrenics. Psychiatry Res 68: 99-109

Benes FM. (2003) Schizophrenia, II: amygdalar fiber alteration as etiology? Am J Psychiatry 160:1053

Berman KF, Zec RF, Weinberger DR (1986) Physiologic dysfunction of dorsolateral prefrontal cortex in schizophrenia. II. Role of neuroleptic treatment, attention, and mental effort. Arch Gen Psychiatry 43:126-135

Bertolino A, Saunders RC, Mattay VS, Bachevalier J, Frank JA, Weinberger DR (1997) Altered development of prefrontal neurons in rhesus monkeys with neonatal mesial temporo-limbic lesions: a proton magnetic resonance spectroscopic imaging study. Cereb Cortex; 7:740-8

Bleuler E, Dementia Praecox or the Group of Schizophrenias, International Universities Press, New York, 1911 (Translated by J. Zinkin, 1950)

Bogerts B (1984) Zur Neuropathologie der Schizophrenien. Fortschr Neurol Psychiatr 52: 428-37

Bogerts B (1993) Recent advances in the neuropathology of schizophrenia. Schizophr Bull 19:431-445

Bouwmeester H, Wolterink G, van Ree JM. (2002) Neonatal development of projections from the basolateral amygdala to prefrontal, striatal, and thalamic structures in the rat. J Comp Neurol 442: 239-49

Broadbelt, K., Byne,W., Jones, L.B (2002). Evidence for a decrease in basilar dendrites of pyramidal cells in schizophrenic medial prefrontal cortex. Schizophrenia Research 58, 75-81

Brockhaus H (1942) Zur feineren Anatomie des Septum und des Striatum. J Psychol Neurol 5:1-56

Buka SL, Fan AP (1999) Association of prenatal and perinatal complications with subsequent bipolar disorder and schizophrenia Schizoph Res 39:113-119

Buxhoeveden DP, Casanova MF (2002b) The minicolumn hypothesis in neuro- science. Brain;125:935-51

Byne W, Buchsbaum MS, Mattiace LA, Hazlett EA, Kemether E, Elhakem SL, Purohit DP, Haroutunian V, Jones L (2002) Postmortem assessment of thalamic nuclear volumes in subjects with schizophrenia. Am J Psychiatry 159: 59-65 
Calhoun ME, Mouton PR (2000) Length measurement: new developments in neurostereology and 3D imagery. J Chem Neuroanar 21:257-265

Cannon TD, Kaprio J, Lonnqvist J, Huttunen M, Koskenvuo M (1998) The genetic epidemiology of schizophrenia in a Finnish twin cohort. A population based modeling study. Arch Gen Psychiatry 55:67-74

Cannon TD (1996) Abnormalities of brain structure and function in schizophrenia: implications for aetiology and pathophysiology Ann Med 28: 533-539

Cannon TD, Mednick SA, Parnas J, Schulsinger F, Praestholm J, Vestergaard A (1993) Developmental brain abnormalities in the offspring of schizophrenic mothers. I. Contributions of genetic and perinatal factors. Arch Gen Psychiatry 50:551-564

Casanova MF, de Zeeuw L, Switala A, Kreczmanski P, Korr H, Ulfig N, Heinsen H, Steinbusch HW, Schmitz $C$ (2005) Mean cell spacing abnormalities in the neocortex of patients with schizophrenia. Psychiatry Res 133:1-12

Casanova MF, Kreczmanski P, Juan Trippe II, Switala A, Heinsen H, Steinbusch HW, Schmizz C (2008). Neuronal distribution in the neocortex of schizophrenic patients. Psychiatry Res 158:267-277

Chance SA, Esiri MM, Crow TJ ( 2002) Amygdala volume in schizophrenia: post-mortern study and review of magnetic resonance imaging findings. Br J Psychiatry 180: 331-8

Church SM, Cotter D, Bramon E, Murray RM ( 2002) Does schizophrenia result from developmental or degenerative processes? J Neural Transm Suppl 63: 129-47

Corselis JA. The pathology of dementia. Br J Psychiatry. 1975;Spec No 9:110-8

Crow TJ (1990) Temporal lobe asymmetries as the key to the etiology of schizophrenia. Schizophren Bull 16: 433-43

Crow TJ (1997) Schizophrenia as failure of hemispheric dominance for language. Trends Neurosci 20: 339-43

Crow TJ (2007) How and why genetic linkage has not solved the problem of psychosis: review and hypothesis. Am J Psychiatry 164:13-21

Cullen TJ, Walker MA, Parkinson N, Craven R, Crow TJ, Esiri MM, Harrison PJ (2003) A postmortem study of the mediodorsal nucleus of the thalamus in schizophrenia. Schizophr Res 60:157-166

Cullen TJ, Walker MA, Eastwood SL, Esiri MM, Harrison PJ, Crow TJ (2006) Anomalies of asymmetry of pyramidal cell density and structure in dorsolateral prefrontal cortex in schizophrenia. Br J Psychiatry 188: 26-31

Cunningham MG, Bhattacharyya S, Benes FM (2002) Amygdalo-cortical sprouting continues into early adulthood: implications for the development of normal and abnormal function during adolescence. J Comp Neurol 453: 116-30

Curtis CE, lacono WG, Beiser M (1999) Relationship between nailfold plexus visibility and clinical, neuropsychological, and brain structural measures in schizophrenia. Biol Psychiatry 46:102-109

Dalman C, Allebeck P, Cullberg J, Grunewald C, Köster M (1999) Obstetrical complications and the risk of schizophrenia. Arch Gen Psychiatry 56:449-456

Danos P, Schmidt A, Baumann B, Bernstein HG, Northoff G, Stauch R, Krell D, Bogerts B (2005) Volume and neuron number of the mediodorsal thalamic nucleus in schizophrenia: a replication study. Psychiatry Res 140:281-9

Davis KL, Stewart DG, Friedman JI, Buchsbaum M, Harvey PD, Hof PR, Buxbaum J, Haroutunian V (2003) White matter changes in schizophrenia: evidence for myelin related dysfunction. Arch Gen Psychiatry 60: 443-56

Dorph-Petersen KA, Pierri JN, Sun Z, Sampson AR, Lewis DA (2004)

Stereological analysis of the mediodorsal thalamic nucleus in schizophrenia: volume, neuron number, and cell types. J Comp Neurol; 472: 449-62 
Dohrenwend BP, Levav I, Shrout PE, Schwartz S, Naveh G, Link BG, Skodol AE, Stueve A (1992) Socioeconomic status and psychiatric disorders: the causation-selection issue. Science 255:946-952

Falkai P, Honer WG, David S, Bogerts B, Majtenyi C, Bayer TA (1999) No evidence for astrogliosis in brains of schizophrenic patients. A post-mortem study. Neuropathol Appl Neurobiol 25:48-53

Fudge JL, Kunishio K, Walsh P, Richard C, Haber SN (2002) Amygdaloid projections to ventromedial striatal subterritories in the primate.Neuroscience 110:257-75

Geddes JR Lawrie SM (1995) Obstetric complications and schizophrenia: a meta-analysis Br J Psychiatry 167:786-793

Gottesman I (1991) Schizophrenia Genesis: the origin of madness. New York: Freeman

Gottesman I (1993) Origins of schizophrenia: past as prologue. In Nature, Nurture and Psychology pp. 231-244 Washington D.C. American Psychological Association

Haber SN, Fudge JL (1997) The interface between dopamine neurons and the amygdala: implications for schizophrenia. Schizophr Bull 23: 471-82

Haefner H, Riecher A, Maurer K, Löffler W, Munk-Jørgensen P, Strömgren E (1989) How does gender influence age at first hospitalization for schizophrenia? A transnational case register study. Psychol Med 19:903-918

Haefner H, Riecher-Roessler A, Hambrecht M, Maurer K, Meissner S, Schmidtke A, Fätkenheuer B, Löffler W, van der Heiden W (1992) IRAOS: an instrument for the assessment of onset and early course of schizophrenia, Schizoph Res 6:209-223

Hakak Y, Walker JR, Li C, Wong WH, Davis KL, Buxbaum JD, Haroutunian V, Fienberg AA (2001) Genomewide expression analysis reveals dysregulation of myclination-related genes in chronic schizophrenia. Proc Natl Acad Sci USA 98:4746-4751

Hambrecht M, Haefner H, Loeffler W (1994) Beginning schizophrenia observed by significant others. Soc Psychiatry Psychiatr Epidemiol 29:53-60

Harrison PJ (1999) The neuropathology of schizophrenia. A critical review of the data and their interpretation. Brain 122:593-624

Harrison PJ (2000) Dialogues in clinical neuroscience. 2-4:349-357

Harrison PJ, Owen MJ (2003) Genes for schizophrenia? Recent findings and their pathophysiological implications. Lancet 361:417-419

Harrison G, Gunnell D, Glazebrook C, Page K, Kwiecinski R (2001) Association between schizophrenia and social inequality at birth: case-control study. $\mathrm{Br} J$ Psychiatry 179:346-350

Harrison PJ, Weinberger DR (2005) Schizophrenia genes, gene expression, and neuropathology: on the matter of their convergence. Mol Psychiatry 10:40-68

Heckers, S.,Heinsen,H.,Heinsen,Y.C., et al (1990) Limbic structures and lateral ventricle in schizophrenia. A quantitative postmortem study. Arch Gen Psych 47:1016-1022

Heinsen H, Heinsen YL (1991) Serial thick, frozen, gallocyanin stained sections of human central nervous system. J Histotechnol 14:167-173

Hill K, Mann L, Laws KR, Stephenson CM, Nimmo-Smith I, McKenna PJ (2004) Hypofrontality in schizophrenia; a meta-analysis of functional imaging studies. Acta Psychiatr Scand 110:243-256

Hirai T, Jones EG (1989) A new parcellation of the human thalamus on the basis of histochemical staining. Brain Res Rev 14:1-34

Honea R, Crow T], Passingham D, Mackay CE (2005) Regional deficits in brain volume in schizophrenia: a meta-analysis of voxel-based morphometry studies. Am J Psychiatry 162: 2233-45 
Hutsler J, Galuske RA (2003) Hemispheric asymmetries in cerebral cortical networks. Trends Neurosci 26(8):429-35

Ingvar DH, Franzen G (1974) Distribution of cerebral activity in chronic schizophrenia. Lancet 21:1484-1486 Jablensky A (2000) Epidemiology of schizophrenia: the global burden of disease and disability. Eur Arch Psych Clin Neurosci 250:274-285

Jablensky A, Sartorius N, Ernberg G, Anker M, Korten A, Cooper JE, Day R, Bertelsen A (1992) Schizophrenia: manifestations, incidence and course in different cultures. A World Health Organization ten-councry study. Psychol Med Monogr Suppl 20:1-97

Johnstone EC, Crow TJ, Firth CD (1976) Cerebral ventricular size and cognitive impairment in chronic schizophrenia. Lancet 30:924-926

Jones EG (1997) A description of the human thalamus. In: Steriade M, Jones EG, McCormick DA (eds) Thalamus, vol. II. Experimental and clinical aspects. Elsevier Science, Oxford, pp 425-500

Jones EG (2000) Minicolumns in the cerebral cortex [letter]. Proc Natl Acad Sci USA 97:5019-21

Jones P, Cannon M (1998) The new epidemiology of schizophrenia. Psychiatr Clin North Am 21:1-25

Kalus, P., Müller, T.J., Zuschratter, W., Senitz, D., 2000. The dendritic architecture of prefrontal pyramidal neurons in schizophrenic patients. NeuroReport 11, 3621-3625

Kemether EM, Buchsbaum MS, Byne W, Hazlett EA, Haznedar M, Brickman AM, Platholi J, Bloom R (2003) Magneric resonance imaging of mediodorsal, pulvinar, and centromedian nuclei of the thalamus in patients with schizophrenia. Arch Gen Psychiatry 60:983-991

Kendell RE, Juszczak E, Cole SK (1996) Obstetrical complications and schizophrenia: a case control study based on standardized obstetrical records. Br J Psychiatry 168:556-561

Kendler KS.(2005) "A gene for ...." The nature of gene action in psychiatric disorders. Am J Psychiatr 162: 1243-52

Kety SS (1959a) Biochemical theories of schizophrenia. I. Science 129:1528-1532

Kety SS (1959b) Biochemical theories of schizophrenia. II. Science 129:1590-1596

Kirov G, O'Donovan MC, Owen MJ. (2005) Finding schizophrenia genes. J Clin Invest 115: 1440-8

Kraepelin E, Dementia Praecox. E. S. Livingstone, Edinburgh, 1919

Kreczmanski P, Schmidt-Kastner R, Heinsen H, Steinbusch HW, Hof PR, Schmiz C (2005) Stereological studies of capillary length density in the frontal cortex of schizophrenics. Acta Neuropathol 109:510-518

Kreczmanski P, Heinsen H, Mantua V, Woitersdorf F, Masson T, Ulfig N, Schmidt-Kastner R, Korr H, Steinbusch HW, Hof PR, Schmitz C (2007). Volume, neuron density, and total neuron number in five subcortical regions in schizophrenia. Brain 130:678-692

Kreczmanski P, Helmut Heinsen, Valentina Mantua et al. Acta Neuropathol. 2009 Apr;117(4):409-21. Epub 2009 Feb 6

Lawrie, S. M. \& Abukmeil, S. (1998) Brain abnormality in schizophrenia. A systematic and quantitative review of volumetric magnetic resonance imaging studies. British Journal of Psychiatry, 172, 110-120

Lauer M, Beckmann H (1997) The human striatum in schizophrenia. I.Increased number of striatal neurons in schizophrenics. Psychiatry Res 68:99-109

Lauer M, Heinsen H (1996) Cytoarchitectonics of the human nucleus accumbens. J Hirnforsch 37:243-254

Lewis DA, Lewitt P (2002) Schizophrenia as a disorder of neurodevelopment. Annu Rev Neurosci 25:409-432

Lieberman JA (1999) Is schizophrenia a neurodegenerative disorder? A clinical and neurobiological perspective. Biol Psychiatry 46:729-739 
Chapter 7

Locke J. An Essay concerning human understanding. London, 1841

Malaspina D, Harkavy-Friedman J, Corcoran C, Mujica-Parodi L, Printz D, Gorman JM, Van Heertum R (2004) Resting neural activity distinguishes subgroups of schizophrenia patients. Biol Psychiatry 56:931-937

McCarley RW, Wible CG, Frumin M, Hirayasu Y, Levitt JJ, Fischer IA, Shenton ME (1999) MRI anatomy of schizophrenia. Biol Psychiatry 45:1099-1119

McClure RK, Lieberman JA (2003) Neurodevelopmental and neurodegenerative hypotheses of schizophrenia: a review and critique. Curr Op in Psych 16 (Suppl):S15-S28

McGlashan, Johannessen JO (1996) Early detection and intervention with schizophrenia: rationale. Schizophr Bull 22;201-222

McGue M, Gottesman II (1989) A single dominant gene still cannot account for the transmission of schizophrenia Arch Gen Psych; 46:478-479

Mednick SA, Machon RA, Huttunen MO, Bonett D (1988) Adult schizophrenia following prenatal exposure to an infuenza epidemic. Arch Gen Psychiatry; 45:189-192

Meyer-Lindenberg A, Miletich RS, Kohn PD, Esposito G, Carson RE, Quarantelli M, Weinberger DR, Berman KF (2002) Reduced prefrontal activity predicts exaggerated striatal dopaminergic function in schizophrenia. Nat Neurosci 5:267-271

Middleton FA, Mirnics K, Pierri JN, Lewis DA, Levitt P (2002) Gene expression profiling reveals alterations of specific metabolic pathways in schizophrenia. J Neurosci 22:2718-2729

Mountcastle VB 1997 The columnar organization of the neocortex.120:701-22

Mouton PR, Gokhale AM, Ward NL, West MJ (2002) Stereological length estimation using spherical probes. J Microsc 206:54-64

Murray RM, Sham P, van Os, Zanelli J, Cannon M, McDonald C (2004) A developmental model for similarities and dissimilarities between schizophrenia and bipolar disorder. Schizoph. Res 71:405-416

Mueser KT, McGurk SR (2004) Schizophrenia. Lancet 363:2063-2072

Nelson MD, Saykin AJ, Flashman LA, Riordan HJ (1998) Hippocampal volume reduction in schizophrenia as assessed by magnetic resonance imaging: a meta-analytic study Arch Gen Psychiatry 57(5):433-40

Owen MJ, Williams NM, Donovan MC (2004) The molccular genetics of schizophrenia: new findings promise new insight. Mol Psych 9:14-27

Pakkenberg B (1990) Pronounced reduction of total neuron number in mediodorsal thalamic nucleus and nucleus accumbens in schizophrenics. Arch Gen Psychiatry 47:1023-1028

Petty RG (1999) Structural asymmetries of the human brain and their disturbance in schizophrenia. Schizophren Bull 25: 121-39

Plum F (1972) Prospects for research on schizophrenia. Neuropathological findings. Neurosci Res Prog Bull 10:384-388

Popken GJ, Bunney WE, Potkin SG, Jones EG (2000) Subnucleus-specific loss of neurons in medial thalamus of schizophrenics. Proc Natl Acad Sci USA 97:9276-9280

Prabakaran S, Swatton JE, Ryan MM, Huffaker SJ, Huang JT, Griffin JL, Wayland M, Freeman T, Dudbridge F, Lilley KS, Karp NA, Hester S, Tkachev D, Mimmack ML, Yolken RH, Webster MJ, Torrey EF, Bahn $S$ (2004) Mitochondrial dysfunction in schizophrenia: evidence for compromised brain metabolism and oxidative stress. Mol Psychiatry 9:684-697

Ross BM, Hughes B, Turenne S, Seeman M, Warsh JJ (2004) Reduced vasodilatory response to methylnicotinate in schizophrenia as assessed by laser Doppler flowmetry. Eur Neuropsychopharmacol 14:191-197 
Sartorius N, Jablensky A, Korten A, Ernberg G, Anker M, Cooper JE, Day R (1986) Early manifestations and first contact incidence of schizophrenia in different cultures. Psychol Med 16:909-926

Sawa A, Snyder SH (2002) Schizophrenia: diverse approaches to a complex disease. Science 296: 692-5

Schmidt-Kastner R, van Os J, Steinbusch HWM, Schmitz C. (2006) Gene regulation by hypoxia and the neurodevelopmental origin of schizophrenia. Schizophr Res 84: 253-71

Schmitz C (2000) Variation of fractionator estimates and its prediction. Anat Embryol 198: 371-97

Schmitz C, Hof PR (2000) Recommendations for straightforward and rigorous methods of counting neurons based on a computer simulation approach. J Chem Neuroanat 20: 93-114

Schmitz C, Hof PR (2005) Design-based stereology in neuroscience. Neuroscience 130: 813-831

Schwab SG, Knapp M, Mondabon S, Hallmayer J, Borrmann-Hassenbach M, Albus M, Lerer B, Rietschel M, Trixler M, Maier W, Wildenauer DB (2003) Support of association of schizophrenia with genetic variation in the 6p22.3 gene, dysbindin, in sib pair families with linkage and in an additional sample of triad families. Am J Hum Genet 72:185-190

Selemon, L.D., Rajkowska, G., Goldman-Rakic, P.S., 1998. Elevared neuronal density in prefrontal area 46 in brains from schizophrenic patients: application of a three-dimensional, stereologic counting method. Journal of Comparative Neurology 392, 402-412

Siever LJ, Davis KL (2004) The pathophysiology of schizophrenia disorders: perspectives from the spectrum. Am J Psychiarry 161:398-413

Shenton ME, Dickey CC, Frumin M, McCarley RW (2001) A review of MRI findings in schizophrenia. Schizophren Res 49:1-52

Stefansson H, Sigurdsson E, Steinsthordottir V, Bjornsdottir S, Sigmundsson T, Ghosh S, Brynjolfsson J, Gunnarsdottir $S$, Ivarsson $O$, Chou TT, Hjaltason O, Birgisdottir B, Jonsson H, Gudnadottir VG, Gudmundsdottir $E$, Bjornsson A, Ingvarsson B, Ingason A, Sigfusson $S$, Hardardottir $H$, Harvey RP, Lai D, Zhou M, Brunner D, Mutel V, Gonzalo A, Lemke G, Sainz J, Johannesson G, Andresson T, Gudbjartsson D, Manolescu A, Frigge ML, Gurney ME, Kong A, Gulcher JR, Petursson H, Stefansson K (2002) Neuregulin 1 and susceptibility to schizophrenia. Am J Hum Genet 71:877-892

Straub RE, Jijang Y, MacLean CJ, Ma Y, Webb BT, Myakishev MV, Harris-Kerr C, Wormley B, Sadek H, Kadambi B, Cesare AJ, Gibberman A, Wang X, O'Neill FA, Walsh D, Kendler KS (2002) Genetic variation in the $6 \mathrm{p} 22.3$ gene DTNBP1, the human ortholog of the mouse dysbindin gene, is associated with schizophrenia. Am J Hum Gener 71:337-348

Susser E, Neugebauer R, Hoek HW (1996) Schizophrenia after prenatal famine: further evidence. Arch Gen Psychiatry 53:25-31

Tamminga CA, Vogel M, Gao X, Lahti AC, Holcomb HH (2000)The limbic cortex in schizophrenia: focus on the anterior cingulate. Brain Res Rev 31: 364-70

Tkachev D, Mimmack ML, Ryan MM, Wayland M, Freeman T, Jones PB, Starkey M, Webster MJ, Yolken RH, Bahn S (2003) Oligodendrocyte dysfunction in schizophrenia and bipolar disorder. Lancet 362:798-805

Tsuang M (2000) Schizophrenia: genes and environment. Biol Psychiat 47:210-220

Vawter MP, Tomita H, Meng F, Bolstad B, Li J, Evans S, Choudary P, Atz M, Shao L, Neal C, Walsh DM, Burmeister M, Speed T, Myers R, Jones EG, Watson SJ, Akil H, Bunney WE (2006) Mitochondrial-related gene expression changes are sensitive to agonal-pH state: implications for brain disorders. Mol Psychiatry 615:663-679 
Weinberger DR (1987) Implications of normal brain development for the pathogenesis of schizophrenia. Arch Gen Psychiatry 44:660-669

Willams NM, Preece A, Spurlock G, Norton N, Williams HJ, Zammit S, O'Donovan MC, Owen MJ (2003) Support for genetic variation in neuregulin 1 and susceptibility of schizophrenia Mol Psychiatry 8:485487

WHO. Schizophrenia. An international follow up study. Wiley Chichester, 1979

WHO, Annual Health Report, 2001

Wolterink G, Daenen LE, Dubbeldam S, Gerrits MA, van Rijn R, Kruse CG, van der Heijden JA, van Ree JM (2001) Early amygdala damage in the rat as a model for neurodevelopmental psychopathological disorders. Eur Neuropsychopharmacol; $11: 51-9$

Wright IC, Rabe-Hesketh S, Woodruff PW, David AS, Murray RM, Bullmore ET (2000) Meta-analysis of regional brain volumes in schizophrenia. Am J Psychiatry 157:16-25

Wyatt RJ (1995) Early intervention for schizophrenia; can the course of the illness be altered ? Biol Psychiarry $38: 1-3$

Young KA, Manaye KF, Liang C, Hicks PB, German DC (2000) Reduced number

of mediodorsal and anterior thalamic neurons in schizophrenia. Biol

Psychiatry 47: 944-53

Zipursky RB, Lim KO, Sullivan EV, Brown BW, Pfefferbaum A (1992) Widespread cerebral gray matter volume deficits in schizophrenia. Arch Gen Psychiatry 49:195-205

Zipursky RB, Seeman MV, Bury A, Langevin R, Wortzman G, Karz R (1997) Deficits in gray matter volume are present in schizophrenia but not bipolar disorder. Schizophr Res 26:85-92 


\section{Summary}

Chapter 1 is a general introduction to this thesis. It elucidates the origins of schizophrenia as a nosological entity, followed by the overview of the current epidemiological, genetical and environmental data and evolution of modern theoretical concepts of the disease. It further explains the consequences of the rapid progress of the technology behind methodology, focusing on post mortem investigations of human brain.

Chapter 2 of this thesis describes morphological alterations of the cytoarchitecture in five subcortical regions of the brain of patients with schizophrenia (caudate nucleus, putamen, nucleus accumbens, mediodorsal nucleus of the thalamus, and lateral nucleus of the amygdala), investigated with state-of-the-art design-based stereological techniques (focussing on volumes, neuron densities and total neuron numbers). The previously unrecognized set of alteration comprises of reduced volumes of the total cortical gray matter, putamen and nucleus accumbens, reduced total neuron numbers in the caudate nucleus, putamen and the lateral nucleus of the amygdala and no correlation between the illness duration of the patients with schizophrenia and the observed neuropathological alterations

Chapter 3 and 4 describe results of investigations on microvasculature abnormalities suggested in the brain of patients with schizophrenia. They present data collected during investigation of variables such as microvessel length density, total microvessel length and microvessel length per neuron in the caudate nucleus, putamen, nucleus accumbens, mediodorsal nucleus of the thalamus, and lateral nucleus of the amygdala followed by assessment of possible changes in total microvessel length and mean microvessel length per neuron in these regions. The patients with schizophrenia showed a statistically significant increase in mean microvessel length density in the caudate nucleus and in the mediodorsal nucleus of the thalamus, and a decrease in mean total microvessel length in the lateral nucleus of the amygdala. There were, however, no significant differences between the patients with schizophrenia and the controls with respect to the mean microvessel length per neuron in all investigated subcortical areas. The analysis of the mean capillary length density within cortical areas 9 and 24 also showed no significant differences between patients with schizophrenia and controls. Only the combination of diagnosis and hemisphere had a significant effect on the mean capillary length density within both areas 9 and 24 .

Chapters 5 and 6 describe minicolumnar abnormalities in the neocortex of the patients with schizophrenia, focussing on alterations in mean cell spacing and in the distribution 
of neurons in the neocortex. Mean cell spacing abnormalities were not significant in posthoc analyses and therefore tentative in nature. It is however possible that the interneuronal mean cell spacing is altered in the brains of patients with schizophrenia. The findings indicate changes rather within different lamina than within the cell core compartment of minicolumns. Moreover, the alterations in mean cell spacing may be secondary to the loss of modulatory input to the cell minicolumns. Therefore, these alterations do not indicate a single mechanism that can be found in all regions but rather a disruption of the systems that modulate information processing, another aspect of the wiring, cytoarchitecture and cell morphology alterations. The neuronal distribution in the neocortex of patients with schizophrenia are consistent with altered neocortical neuropil reports, a phenomenon well established in the literature. These alterations reflect de facto modulatory systems abnormalities innervating the cell minicolumns.

Finally, in the last chapter, results presented in this thesis are discussed with respect to the application of modern theoretical concepts in current post mortem investigations of schizophrenia. 


\section{Streszczenie}

Rozdział pierwszy zawiera wstęp niniejszej pracy doktorskiej, w którym na pierwszym miejscu przedstawiono zarys historii schizofrenii oraz proces formowania podstawowych pojęć odnoszących się do psychopatologii choroby. Następnie dokonano przeglądu podstawowych danych epidemiologicznych, genetycznych oraz środowiskowych. Rozdział ten kończy się opisem stanu zaawansowania technologicznego w badaniach post mortem z uwzględnieniem metodologii zastosowanej w tej pracy.

W rozdziale drugim umieszczono publikację wyników badań stereologicznych cytoarchitektury pięciu podkorowych struktur mózgowia, elementów prążkowia (jądro ogoniaste, skorupa oraz jądro półleżące), przyśrodkowo-grzbietowego jądra wzgórza oraz jądra bocznego ciała migdałowatego. Mózgowia schizofreników charakteryzowały się zmniejszeniem się całkowitej objętości korowej istoty szarej, skorupy oraz jądra półleżącego, zmniejszoną całkowitą ilością neuronów jądra ogoniastego, skorupy, oraz jądra bocznego ciała migdałowatego. Nie wykazano zależności pomiędzy czasem trwania choroby, liczonym od postawienia rozpoznania do momentu zgonu, a dynamiką zmian parametrów morfologicznych względem czasu.

Rozdziały trzeci i czwarty zawierają opublikowane wyniki badań nad unaczynieniem mózgowia na poziomie kapilar (o średnicy mniejszej niż $9 \mu \mathrm{m}$ ). Zebrane dane obejmują parametry takie, jak gęstość długości naczyń, całkowitą długość naczyń w odniesieniu do objętości badanej struktury oraz długość naczynia przypadająca na jeden neuron w strukturach takich jak jądro ogoniaste, skorupy, jądro półleżące, przyśrodkowo-grzbietowe jądro wzgórza oraz boczne jądro ciała migdałowatego. Badania wykazały statystycznie znamienną większą średnią gęstość naczyń w jądrze ogoniastym oraz we wzgórzu oraz zmniejszoną całkowitą długość naczyń w jądrze bocznym ciała migdałowatego u schizofreników. Co ciekawe, badania nie wykazały znaczących statystycznie różnic grupy kontrolnej względem schizofreników dla parametrów takich, jak średnia długość naczynia przypadająca na jeden neuron we wszystkich badanych strukturach. Analiza średniej gęstości długości naczyń w polach korowych Brodmanna 9 oraz 24 równiez nie wykazała znaczących statystycznie różnic pomiedzy oba grupami.

Rozdziały piąty oraz szósty opisują wyniki badań nad jednostką funkcjonalną kory nowej: minikolumną. Obejmują one badania średniej odległości pomiędzy neuronami oraz rozkładu przestrzennego neuronów w obrębie jednej minikolumny. Zmiany średniej od- 
ległości pomiędzy neuronami nie były znaczące statystycznie i po przeprowadzeniu analizy post-hoc i podlegają dalszej dyskusji i interpretacji. Nie jest jednakże niemożliwe, że średnia odległość pomiędzy neuronami w mózgu schizofrenika rożni się od tej w zdrowym mózgu. Wyniki wskazują, że zmiany znajdują się raczej w obrębie poszczególnych warstw kory, niż na całej długości minikolumny. Co więcej, zmiany średniej odległości między neuronami mogą być wtórne do utraty impulsów modulacyjnych w komórek nerwowych w obrębie minikolumny. Tego typu zmiany nie wskazują na jeden, uogólniony mechanizm, którego należałoby poszukiwać we wszystkich strukturach mózgowia, ale raczej na zakłócenie pracy systemów neuronalnych modulujących przetwarzanie informacji, innej konfiguracji sieci połączeń, cytoarchitektury oraz morfologii neuronów. Rozkład neuronów kory nowej schizofreników opublikowanych $\mathrm{w}$ niniejszej pracy odpowiada zmianom zaobserwowanym w neuropilu schizofreników, zjawisku dobrze znanym z literatury. Zmiany te odzwierciedlają de facto zmiany stystemów modulujących w sieci neuronalnej minikolumny.

Ostatni rozdział zawiera dyskusję na temat wyników zawartych w powyższych publikacjach $\mathrm{z}$ uwzględnieniem i zastosowaniem aktualnych poglądów obecnych $\mathrm{w}$ środowisku badań post-mortem nad schizofrenią. 


\section{Thanks}

At first I'd like to thank to my family. To my parents: statistically seen I was given this one single possibility of existence in a hazy January day, somewhere in 1975 which resulted in an outcome at 23rd of October the same year. Sister/Siostro: Wielkie Dzięki - Great Thanks, Brother/Bracie: Dzięki za upierdliwość - thanks for being pain in the a...

Christoph, needless to say. If I'd said that this was not possible without your support, advice and time, it would be not even - definitely - not enough

Harry, great thanks for your support, logistics, time with space, and possibility to work in your Institute.

Professor Hof, without your critical views and comments it wouldn't be possible in a shape as it is.

I would like to thank professor Ciszek, head of Department of Anatomy at the Warsaw Medical University. Without the support of this very wise man I wouldn't be where I am right now (and you reading this).

Dear Dr Wierzba-Bobrowicz, thank you sincerely and very much for your support, comments, good word, faith and humor during those long years.

Dear professor Korr, time spend in your lab was one of the cheerful times in my life.

I would like especially to thank Helen Steinbusch for her rays of light in everyday work, humor and attitude, and Marianne for her grace.

Kowalu, dzięki.

Wujek, bedzie dobrze.

Bart, thanks for help, dude.

Eva, thanks for keeping my keys for so long :D

My dear Ladies, Marijke, Annerieke, Imke and Eveline, I am lost in translation. 


\section{Curriculum vitae}

Paweł Kreczmański was born in Warsaw in 1975. After he finished basic school in 1990, he attended Lyceum "Hugo Kolłątaj" in Warsaw where he graduated in 1994. He was then successfully admitted to study medicine at Warsaw Medical University. Six years later he started to work as a young assistant at the Anatomy Department of the Warsaw Medical University. During this time he also worked at the Central Hospital of the Ministry of the Internal Affairs during his post-graduate training for one year. In 2003 he went to Rostock to study the development of the human fetal brain at the University of Rostock in Germany under supervision of prof. Norbert Ulfig. After three months, in June of 2003 the was offered a $\mathrm{PhD}$ position at joined project between Aachen University and Maasticht University under supervision of dr. Christoph Schmitz until the end of 2006. This thesis is the result of that project. After that he worked for one year at the Department of the Neurosurgery at Maastricht University Hospital and one year at the Department of Pathology at Maastricht University Hospital. He is currently working as a doctor in a nursery home in Landgraaf searching for other opportunities. He likes philosophy, history and diplomacy. 


$\begin{aligned} \text { Name } & \text { Paweł Kreczmański }(\mathrm{m}) \\ \text { Date of birth } & 23-10-1975 \text { (33 years) } \\ \text { Address } & \text { Grote Staat 16 C05, 6211 CW Maastricht } \\ \text { Country } & \text { Netherlands } \\ \text { Telephone } & +31(0) 617678855 \\ \text { E-mail } & \text { pawel.kre@NP.unimaas.nl } \\ \text { Citizenship } & \text { Polish }\end{aligned}$

\section{Education}

1994-2000 Medical University of Warsaw in Warsaw, medical degree

1990-1994 Hugo Kołłątaj Lyceum, Warsaw 10 subjects: Polish, German, English, Russian, Mathematics, Biology, Chemistry, Physics, Geography, History

\section{Occupation}

January 2009 - present

January 2008 - December 2008

Medical doctor at the nursery home De Dormig, Meander Group, Landgraaf, Netherlands Resident Pathology, Department of Pathology at Maastricht University Medical Centre, Maastricht

January 2007 - December 2007 Assistant Neurosurgery, Maastricht University Medical Centre, Maastricht

October 2003 - December2006 PhD Student. Project Neuropathology of Schizophrenia, Dept. of Psychiatry and Neuropsychology, division Cellular Neuroscience, University Maastricht, Maastricht

Maart 2003 - Oktober 2003 "Wissenschaftlicher Angestellter" (equivalent of the Post-Doc), Department Anatomy, University Rostock, Germany

October 2001 - March 2003 Young-Assistant Department Anatomy, Medical University of Warsaw

October 2000 - Oktober 2001 Post graduate training Central Hospital of the Ministry of the Internal Affairs, Warsaw, Poland

\section{Trainings}




\section{List of publications}

1. Kreczmanski P, Heinsen H, Mantua V, Woltersdorf F, Masson T, Ulfig N, SchmidtKastner R, Korr H, Steinbusch HWM, Hof PR and Schmitz C. Capillary length density, total capillary length and capillary length per neuron in five subcortical regions in schizophrenia Acta Neuropathol, Apr;117(4):409-21. Epub 2009 Feb 6. IF 3.179 (2007)

2. Kreczmanski P, Heinsen H, Mantua V, Woltersdorf F, Masson T, Ulfig N, SchmidtKastner R, Korr H, Steinbusch HWM, Hof PR and Schmitz C. Volume, neuron density, and total neuron number in five subcortical regions in schizophrenia. Brain. 2007 Mar;130(Pt 3):678-92. Epub 2007 Feb 15. IF 8.568 (2007)

3. Kreczmanski P, Schmidt-Kastner R, Heinsen H, Steinbusch HWM, Hof PR, Schmitz C. Stereologic studies of capillary length density in the frontal cortex of schizophrenics. Acta Neuropathol 2005;109:510-518. IF 2.527 (2005)

4. Casanova MF, Kreczmanski P, Trippe J 2nd, Switala A, Heinsen H, Steinbusch HW, Schmitz C. Neuronal distribution in the neocortex of schizophrenic patients. Psychiatry Res. 2008 Apr 15;158(3):267-77. Epub 2008 Feb 20. IF 2.298 (2007)

5. Casanova MF, deZeeuw L, Switala A, Kreczmanski P, Korr H, Ulfig N, Heinsen H, Steinbusch HWM, Schmitz C. Mean cell spacing abnormalities in the neocortex of patients with schizophrenia. Psychiat Res 2005;133:1-12. IF 1.957 (2005)

6. Yamamoto H, Hofmann S, Hamasaki DI, Yamamoto H, Kreczmanski P, Schmitz C, Parel JM, Schmidt-Kastner R. Wolfram syndrome 1 (WFS1) protein expression in retinal ganglion cells and optic nerve glia of the cynomolgus monkey. Exp Eye Res. 2006 Nov;83(5):1303-6. Epub 2006 Aug 22. IF 2.651 (2006)

\section{Presentations}

\section{Poster presentations}

14 November, 2005: The Society for Neuroscience, 35th Annual Meeting Washington, DC: Stereologic studies of capillary length density in the frontal cortex of schizophrenics Kreczmanski P, Heinsen H, Mantua V, Woltersdorf F, Masson T, Ulfig N, Schmidt-Kastner R, Korr H, Steinbusch HWM, Hof PR and Schmitz C

\section{Oral presentations}

11 April 2008, Institute's day, Maastricht, Annual Institute for Neuroscience Meeting, Division 2: Capillary length density, total capillary length and capillary length per neuron in five subcortical regions in schizophrenia: Kreczmanski P, Heinsen H, Mantua V, Woltersdorf F, Masson T, Ulfig N, Schmidt-Kastner R, Korr H, Steinbusch HWM, Hof PR and Schmitz C. Winner of the Second Division Prize for the best publication in 2007. 


\section{Languages}

$\begin{array}{lll}\text { Language } & \text { in writing } & \text { in reading } \\ \text { Polish } & \text { Mother tongue } & \text { Mother tongue } \\ \text { Dutch } & \text { Good } & \text { Good } \\ \text { Engels } & \text { Fluid } & \text { Fluid } \\ \text { Duits } & \text { Basic } & \text { Basic } \\ \text { Russisch } & \text { Basic } & \text { Basic }\end{array}$

\section{Computer skills}

Windows 95/98/ME/XP

Microsoft Office 97/2000/2003 (Word, Excel, PowerPoint, Outlook etc.)

Stereoinvestigator

SPSS, GraphPad

Corel suite 11

Adobe Photoshop 7.0

\section{Hobbies}

History of Europe

Film

Diplomacy 